Portland State University

PDXScholar

Spring 6-2-2015

\title{
An Investigation of the Role of Alternate Numeration Systems in Preservice Teacher Mathematics Content Courses
}

Jodi I. Fasteen

Portland State University

Follow this and additional works at: https://pdxscholar.library.pdx.edu/open_access_etds

Part of the Educational Methods Commons, and the Mathematics Commons Let us know how access to this document benefits you.

Recommended Citation

Fasteen, Jodi I., "An Investigation of the Role of Alternate Numeration Systems in Preservice Teacher Mathematics Content Courses" (2015). Dissertations and Theses. Paper 2314.

https://doi.org/10.15760/etd.2311

This Dissertation is brought to you for free and open access. It has been accepted for inclusion in Dissertations and Theses by an authorized administrator of PDXScholar. For more information, please contact pdxscholar@pdx.edu. 
An Investigation of the Role of Alternate Numeration Systems in Preservice Teacher Mathematics Content Courses

by

Jodi I. Fasteen

A dissertation submitted in partial fulfillment of the requirements for the degree of

\author{
Doctor of Philosophy \\ in \\ Mathematics Education
}
Dissertation Committee:
Eva Thanheiser, Chair
Sean Larsen
John Caughman
Andrea Goforth

Portland State University

2015 
(C) 2015 Jodi I. Fasteen 


\begin{abstract}
Alternate numeration systems are common in preservice teacher (PST) mathematics curricula, but there is limited research on how to leverage alternate systems to promote the development of mathematical knowledge for teaching. I analyzed the role of alternate numeration systems in three ways. I conducted a thematic analysis of current PST textbooks to consider the role of alternate numeration systems in written curricula. I conducted a teaching experiment to analyze PSTs' mathematical activity as they engaged with a base five task sequence to reinvent an algorithm for multiplication. And I introduced problematizing mathematical contexts as a design heuristic, situating this within the design theory of Realistic Mathematics Education. I found that alternate numeration systems can be leveraged to create opportunities for PSTs to (a) engage in guided reinvention of an algorithm, (b) improve understanding of base ten by comparing it to other numeration systems, and (c) reflect on their learning experience and the learning experiences of children.
\end{abstract}




\section{Dedication}

To my son, Henry, who always shares his mathematical thinking with me and to my daughter, Hazel, who wants to be a grad student, just like mom. 


\section{Acknowledgments}

This dissertation study would not have been possible without the support of mentors, colleagues, and family. First, I must acknowledge the influential role of my advisor, Dr. Eva Thanheiser, who was very generous with her time and her advice. She offered countless suggestions for improving ideas, digging deeper into the data, and focusing on the "so what" question at the heart of research. I would also like to thank the rest of my committee. I am grateful to Dr. Sean Larsen, who shared his expertise in the design theory of Realistic Mathematics Education, and to Dr. John Caughman and Dr. Andrea Goforth, who shared their time and support. I also offer my thanks to the research participants, who shared their time and their mathematical thinking, being brave enough to share ideas that were still under development.

I would like to thank my colleagues in the Mathematics and Statistics Department at Portland State University, who offered support and encouragement throughout this

process. In particular, I want to thank my friend and colleague, Kathleen Melhuish. During the research process she was a constant sounding board with insightful contributions and during the writing process she read endless drafts with infinite patience and kind advice. Most of all, she is an amazing friend, and I am grateful.

Finally, I would like to express my gratitude to my family and friends. I am thankful for the words of encouragement from my parents and friends, especially in the final weeks of writing. I am grateful for my husband, who showed great patience as I finished the writing process, and my children, who help me to keep life in perspective, balancing academic pursuits and the amazing experience of motherhood. 


\section{Table of Contents}

Abstract


List of Tables

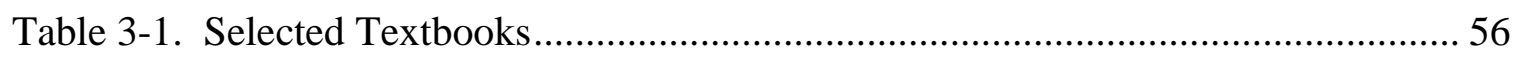

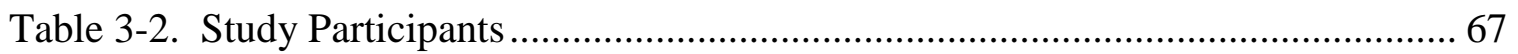

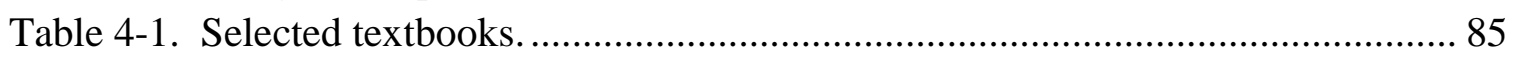

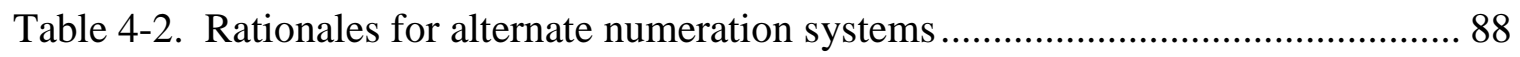

Table 4-3. Codes for analysis of alternate numeration systems.................................. 92

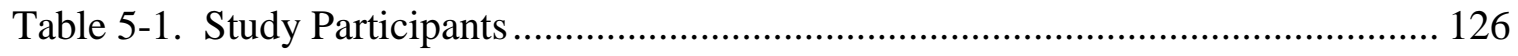




\section{List of Figures}

Figure 2-1. A domain map for mathematical knowledge for teaching. Image from Hill, Ball, \& Schillig (2008, p.377)................................................................................. 15

Figure 2-2. The Ancient Egyptian numeration system is an additive numeration system where symbols indicate their value regardless of their location in a number. 19

Figure 2-3. Six different conceptions for the number 53, with the conceptions in the bottom row highlighting subsets of ten within the total of 53. (Image adapted from Fuson

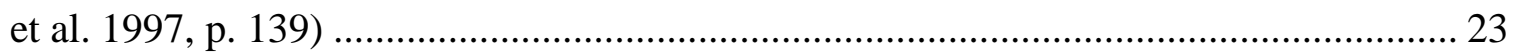

Figure 2-4. Larger units are built out of smaller units without losing the underlying structure of the smaller units (image taken from Kamii, 1994, p.10)............................. 24

Figure 2-5. The repeated addition and area models are represented visually.................. 30

Figure 2-6. The area model is split into sub-areas which are easier to calculate............. 31 Figure 2-7. The standard U.S. algorithm for multiplication, using the example 14 x 23. 32 Figure 2-8. U.S. teachers focused on the procedural error, rather than the student's

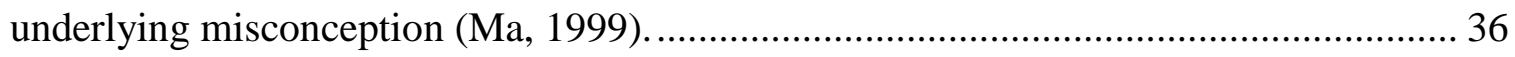

Figure 2-9. An introduction to base five language and imagery.................................. 39 Figure 2-10. The Mayan and base five number systems are two examples of alternate numeration systems (Bennett et al., 2011)................................................................... 40 Figure 2-11. In the base twenty Mayan context, a dot followed by one shell refers to one group of 20, a dot followed by two shells refers to one group of $20^{2}$, or 400 , and dot

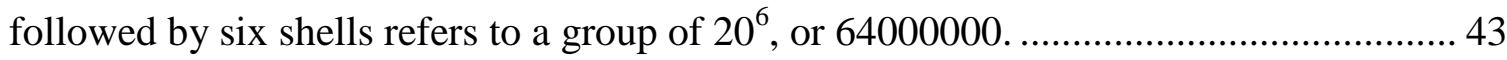
Figure 2-12. The emergent perspective coordinates the social and psychological

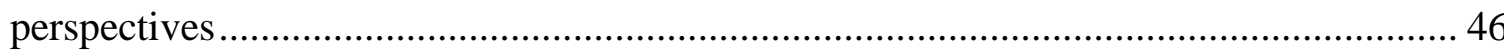
Figure 2-13. Phenomenological analysis of the area model reveals the utility of the model

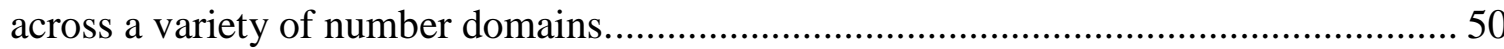

Figure 3-1. Phases of thematic analysis, Image from Braun \& Clarke, 2006, p. 87........ 57

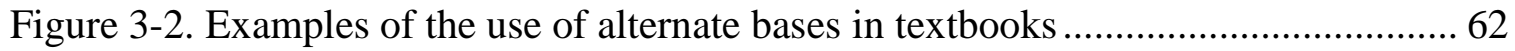

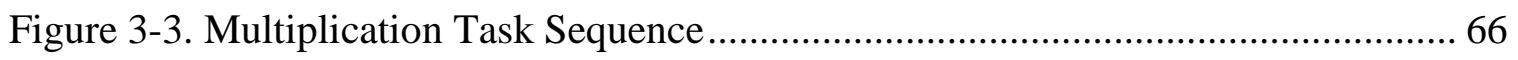

Figure 3-4: Phases of retrospective analysis, with the first two phases repeated for each cycle and the latter two phases conducted across multiple cycles..................................69

Figure 3-5. Example of an episode where preservice teachers are asked to define multiplication in language appropriate for children.............................................. 70 Figure 4-1. Different directions - One text advocates not thinking about base ten while the other draws explicit comparisons between base ten and the alternate base............... 76 Figure 4-2. An example of the base five number system and a general number system. . 78 Figure 4-3. Domains of Mathematical Knowledge for Teaching. image adapted from Ball et al. (2008, p.403) 
Figure 4-4. PSTs are asked to work within base six to make sense of the base six system (Bassarear, 2012a, p. 43)........................................................................................ 90

Figure 4-5. Five historic systems were compared in a single chart (Musser et al., 2011, p. 66). 101

Figure 4-6. An example of comparing between numeration systems by focusing on converting. In this example, 824 is converted to base five (Billstein et al., 2012, p.61).

Figure 4-7. An example of a within base operation: adding two numbers in base four (Sowder et al., 2014, p. 37).

Figure 4-8. Connecting alternate base activities with the MKT framework. The blue boxes relate to comparison activities and the green boxes relate to immersion activities.

Figure 5-1. Base five language and description................................................... 120

Figure 5-2. Five different area visualizations are provided for 12 x 23, highlighting different aspects of multiplication. ................................................................... 121

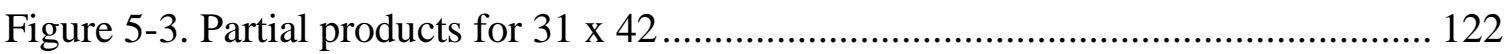

Figure 5-4. Connecting partial products strategies to standard algorithms for

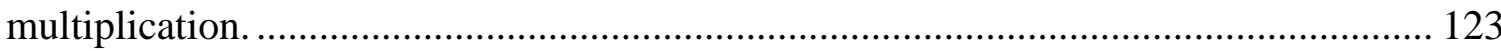

Figure 5-5. Multiplication Task Sequence .............................................................. 128

Figure 5-6. Phases in analysis .............................................................................. 130

Figure 5-7. Example of an episode where preservice teachers are asked to define multiplication in language appropriate for children.....

Figure 5-8. The co-emergence of models of meaning and models of strategy highlights the interplay between models.

Figure 5-9. Wendy and Eli built 3 groups of manipulatives, with 2 longs 3 ones per

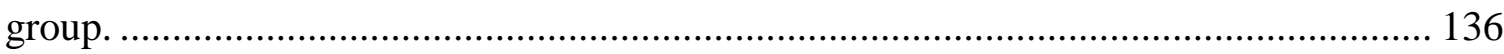
Figure 5-10. Wendy represented $10_{\text {five }} \times 23_{\text {five }}$ by creating five piles of $23_{\text {five }}$............. 138 Figure 5-11. Sarah and Nora recorded strategies for making sense of why long $\mathrm{x}$ long = flat. These strategies leveraged both repeated addition and area interpretations for the meaning of multiplication.

Figure 5-12. Wendy and Eli measured the rectangle in base five, creating an overly thick border.

Figure 5-13. Eli and Wendy determined the area of the rectangle, finding a value 25 times larger than appropriate. 143

Figure 5-14. Wendy and Eli modified their prior image of $420_{\text {five }} \times 310_{\text {five }}$ to match $42_{\text {five }}$ x $31_{\text {five }}$, scribbling over the word "flat" to change the unit type for each subsection. .... 147 Figure 5-15. Nora and Sarah's general strategy for partial products based on rows ..... 150 Figure 5-16. Nora and Sarah's written work displayed a strategy of splitting the rectangular image into quadrants to create four partial products. 
Figure 5-17. Different versions of the partial products model highlight different ways of splitting the area model into subsections.

Figure 6-1. Three shapes are presented as potential examples of triangles on a sphere. Students may investigate geometry on a sphere to better understand planar geometry. 155 Figure 6-2. Definition of the design heuristic problematizing a mathematical context. 157 Figure 6-3. The Times Base Task provides an opportunity to consider the multiplicative structure of place value systems. 


\section{Chapter 1 : Rationale and Introduction}

In Chapter 1, I argue that (a) whole number and operations are at the core of elementary mathematics, (b) children are capable inventors of sophisticated whole number strategies, (c) teachers need specialized mathematical knowledge for teaching (MKT) in order to support children's invention of strategies, and (d) preservice teachers (PSTs) don't have sufficient MKT. After establishing the problem, I argue that alternate bases provide an opportunity for PSTs to develop MKT and I situate the three components of my study within the arena of alternate bases. The first component of the study examines trends in how alternate numerations systems are used in current PST mathematics curricula. The second component of the study establishes a local instructional theory which leverages alternate bases as a teaching tool for multiplication. The third component of the study introduces the design heuristic problematizing mathematical contexts, in which alternate base contexts are considered as an example of wider phenomenon of leveraging non-routine contexts to make sense of routine contexts.

\section{Whole Number and Operations}

Number is a rich, many-sided domain whose simplest forms are comprehended by very young children and whose far reaches are still being explored by mathematicians. Proficiency with numbers and numerical operations is an important foundation for further education in mathematics and in fields that use mathematics. (Kilpatrick, Swafford, \& Findell, 2001, p. 2)

Whole number and operation form the core of elementary mathematics

(Verschaffel, Greer, \& De Corte, 2007). Standards documents such as the Common Core State Standards for Mathematics (CCSS-M) (National Governors Association Center for Best Practices \& Council of Chief State School Officers, 2010) and Principles and 
Investigating Alternate Numeration Systems

Standards for School Mathematics (National Council of Teachers of Mathematics, 2000)

place considerable emphasis on reasoning about whole number and operations. CCSS-M advocates that elementary mathematics education include opportunities for students to use "strategies based on place value, properties of operations, and/or relationships between [operations]" (2010, p. 16).

\section{Children as Capable Inventors}

Prior research on children's mathematical thinking reveals that when children are given appropriate support, they are capable inventors of an array of strategies for multidigit calculations (Ambrose, Baek, \& Carpenter, 2003; Fuson et al., 1997; van Putten, Brom-Snijders, \& Beishuizen, 2005). Appropriate support includes (a) opportunities to investigate contextually based story problems with the intention of building strategies rather than practicing procedures and (b) opportunities to share and compare invented strategies.

While research has shown that children are capable inventors and current education policy encourages sense-making strategies, this is not the type of classroom environment children have traditionally experienced. For example, in my work with PSTs, most describe their childhood mathematics experiences as following the traditional pattern of memorizing and practicing rules that they didn't understand. Fuson warns that this type of traditional classroom experience may encourage the development of rulebased procedural skills rather than the development of appropriate place value conceptions (1992). In particular, standardized algorithms for multidigit operations tend to abbreviate substeps, concealing the sophisticated role of place value. 
In these algorithms, the meaning and scaffolding of substeps have been sacrificed to using a small amount of paper. The multiplication and division algorithms use aligning methods that keep the steps organized by correct place value without requiring any understanding of what is actually happening with the ones, tens, and hundreds. (Fuson, 2003, p. 302)

Kamii and Dominick (1997) and Pesek and Kirshner (2000) warn about the dangers of teaching algorithms without understanding. They indicate that supplying prebuilt standardized algorithms to children may unteach ideas of place value and number sense, while encouraging children to abandon their own mathematical strategies (Kamii \& Dominick, 1997). Pre-built algorithms shift the mathematical authority away from sense-making and toward teacher or textbook provided solution strategies. Children who use non-standard strategies, such as their own invented strategies, are more likely to be able to justify their methods (Carroll, 1999).

In order to support children in inventing sense-making strategies, teachers must have mathematical knowledge that extends beyond procedural fluency. Teachers should be aware of models that support student thinking and they should be able to judge the validity and efficiency of student generated strategies (Ball, Hill, \& Bass, 2005; H. C. Hill, Ball, \& Schillig, 2008). This role of supporting student mathematicians suggests that teachers should have a specialized mathematical knowledge specific to the domain of teaching mathematics.

\section{Mathematical Knowledge for Teaching}

The professional holds knowledge, not only of how - the capacity for skilled performance - but of what and why. The teacher is not only a master of procedure, but also of content and rationale, and capable of explaining why something is done. (Shulman, 1986, p.13) 
Investigating Alternate Numeration Systems

In his 1986 article, Shulman distinguishes pedagogical content knowledge from content knowledge, indicating that pedagogical content knowledge is subject matter knowledge that is specialized for teaching, such as knowledge of representations, examples, and analogies that are helpful to teaching, as well as knowledge of where students struggle and common preconceptions. Shulman advocated for research about pedagogical content knowledge. In response to this and other calls for research, Ball, Hill, and Bass (2005) developed the construct of mathematical knowledge for teaching (MKT) to refer to the specialized knowledge that mathematics teachers should have. Similarly, Ma (1999) argues:

Certainly a teacher's subject matter knowledge of mathematics differs from that of a non-teaching person. Special features of a teacher's subject matter knowledge are derived from the task of promoting student learning. To facilitate learning, teachers tend to make explicit the connections between and among mathematical topics that remain tacit for nonteachers. (Ma, 1999, p. 21)

Researchers have shown that teachers' MKT influences their ability to support student learning and that many teachers lack sufficient MKT (Ball, 1990b; Ball et al., 2005; Ma, 1999). Preservice mathematics content courses provide an opportunity to help PSTs develop a deeper understanding of the mathematics they will teach.

\section{Preservice Teacher Knowledge}

Prior research on PSTs mathematical knowledge has tended to focus on what they know and don't know, rather than how they learn (Mewborn, 2001; Thanheiser et al., 2014). Mewborn (2001) uses the term snapshot to refer to studies that (a) examine what a subject knows at a particular point in time or (b) focus on pre and post data about student knowledge. A 2014 study by Thanheiser et al. indicates that out of 112 journal 
Investigating Alternate Numeration Systems

articles about PST content knowledge, published between 1978 and 2012, 104 of the studies were snapshot or static studies of knowledge, while only 8 of the studies focused on motion studies of learning. In the content domain of whole number and operation, only 2 out of 26 studies were about PSTs learning, while the other 24 were static studies of knowledge. Mewborn (2001) and Thanheiser et al. (2014) advocate for more studies which focus on the development of PST knowledge. In my study I address this gap by examining the mathematical activity of PSTs as they reinvent a general strategy for multidigit multiplication in base five.

In their summary of 112 studies of PST knowledge, Thanheiser et al. (2014) point to the consistent trend that PSTs tend to rely on procedures rather than underlying concepts. PSTs are able to use procedures that they are not able to explain (Ball, 1990b; J.-J. Lo, Grant, \& Flowers, 2008; Ma, 1999; Thanheiser, 2009a, 2010). Moreover, PSTs may be unaware that there exists a why behind commonly used mathematical procedures (Ball, 1990a; J.-J. Lo et al., 2008; Thanheiser, Philipp, Fasteen, Strand, \& Mills, 2013). PSTs may believe that knowing how to do the procedures is equivalent to understanding (Graeber, 1999).

While mathematics education researchers are aware of problematic issues with PSTs' content knowledge, the PSTs may be unaware of their own gaps in knowledge. Thanheiser (2009a) notes that of the 15 PSTs surveyed in her study, 14 felt they already knew enough mathematics to teach $3^{\text {rd }}$ grade, prior to their first math content course. Moreover, "When faced with their lack of mathematical understanding, PSTs are often unperturbed, because they hold a self-perpetuating belief that 'if I, a college student, do 
not know something, then children would not be expected to know it, and if I do know something, I certainly don't need to learn it again' (Philipp et al., 2007, p. 439).”

\section{Genuine Problems}

Given that PSTs lack MKT, but believe that they know enough to teach, teacher educators have their hands full. Teacher educators need tasks that are genuine problems for PSTs, problems which provoke intellectual curiosity. Harel (2007) captures this need in his necessity principle for instruction, which states that students must be motivated by intellectual need/curiosity in order to be open to learning what a teacher intends them to learn. In order for a situation to provide an intellectual need, it must be a genuine problem $^{1}$ to the student. Novel or non-routine contexts, such as alternate bases, provide a tactic for provoking PST curiosity (McClain, 2003; Yackel, Underwood, \& Elias, 2007).

In this study I investigate the role of alternate bases in current PST textbooks and I examine PSTs' mathematical activity with an instructional sequence for multidigit multiplication in an alternate base context. I also discuss a task design heuristic of problematizing mathematical contexts more generally. Alternate bases are not selected merely because they are novel, as novelty is likely not a sufficient reason to engage with content. Alternate bases serve as a non-routine version of an overly routine context, the base ten system (McClain, 2003). PSTs are asked to step back from this routine system and imagine themselves as children, learning a number system for the first time. While PSTs are not in the same position as children, the invitation to imagine the learning

\footnotetext{
${ }^{1}$ By genuine problems, I mean problems for which PSTs do have an accessible entry point but do not have predetermined solution strategies (Kolovou, Van den Heuvel-Panhuizen, \& Bakker, 2009).
} 
process of children can serve to spark the curiosity of PSTs. The alternate base investigation serves the dual purposes of (a) encouraging PSTs to imagine how their future students will make sense of a place value system (both challenges and ah-ha moments) and (b) providing an opportunity for PSTs to develop a more sophisticated view of place value number systems.

As long as the [student] operates with the decimal system without having become conscious of it as such, he has not mastered the system but is, on the contrary, bound by it. When he becomes able to view it as a particular instance of the wider concept of a scale of notation, he can operate deliberately with this or any other numerical system. (Vygotsky, 1962, p. 115)

As Vygotsky indicates, looking across different numeration systems may lead to the development of a generalized conception of place value structure. In Chapter 6, I focus on shifting to alternate bases as an example of a design heuristic for problematizing mathematical contexts. In the following section I provide a brief overview of the mathematics of alternate bases.

\section{Alternate Bases}

An alternate base number system is a positional number system which is based on a number other than ten. In the base ten number system, each time you collect ten of one type of unit, those units can be grouped together into the next larger unit. That is, ten ones can be regrouped as one ten, ten tens can be regrouped as one hundred, and so on. Each column in a base ten numeral indicates the quantity of units of a particular type, with the columns having the values of $10^{0}, 10^{1}, 10^{2}$, etc. The decision to use ten as the regrouping quantity is an artifact of cultural convention, likely based on the number of fingers on two hands. We use base ten because our parents and grandparents did. 
Historically, other numbers have been selected as the regrouping quantity, such as Mayan base 20 and Babylonian base 60 . Our system of time, with 60 seconds per minute and 60 minutes per hour, is an artifact of this historic system. In the Babylonian system, rather than having a ones place, tens place, and hundreds place, there was a ones place, $60 \mathrm{~s}$ place, and 3600s ( or $60^{2} \mathrm{~s}$ ) place. Additional larger place values can be found by computing larger powers of the base.

Alternate bases are already present in many textbooks used for PST mathematics content courses (e.g. Bassarear, 2012c; Bennett, Burton, \& Nelson, 2012a). Alternate bases may be used for a variety of pedagogical or mathematical goals and one aspect of my study is an analysis of the roles of alternate bases in PST mathematics curricula. Textbooks use both historic number systems, such as Mayan and Babylonian, and modern notation systems, such as base five, as examples of alternate base systems. Examples are provided in Chapter 2.

McClain (2003), Yackel, Underwood, \& Elias (2007), and Roy (2014) provide evidence of the success of alternate bases as a context to promote PST exploration and sense-making about whole number concepts, creating instructional sequences for addition and subtraction. I focus on the development of an instructional sequence for multidigit multiplication in base five. There are several reasons I have selected multiplication as the operation of focus (besides the lack of prior research on alternate base multiplication). The place value system has a multiplicative structure, where each place value is a constant multiple of the place value to its right. Studying multiplication in an alternate base may help PSTs to make sense that (a) there is a multiplicative structure in positional 
Investigating Alternate Numeration Systems

number systems and (b) that multiplicative structure can be leveraged for creating strategies.

\section{Purpose of My Study}

In the prior sections of this chapter, I have established that children are capable inventors in the context of whole number and operation and that teachers require MKT in order to support children as they build a conceptual understanding of number and operation. One strategy to help PSTs to develop the necessary MKT is to use the nonroutine context of alternate bases. In this study I approach the arena of alternate bases with three distinct questions:

(1) How and why are alternate numeration systems being used in PST textbooks?

(2) How can alternate bases be leveraged to support PSTs in reinventing a general strategy for multiplication?

(3) How can problematizing to an alternate base be considered as an example of a wider task design heuristic?

To answer the first question, I conducted a textbook analysis of a selection of current PST textbooks, focusing on the role and purpose of alternate bases. I built a framework for the role of alternate bases as a teaching tool in PST mathematics content courses.

To address the second question, I built a base five multiplication sequence (See Chapter 3) which I tested, analyzed, and modified during two cycles of a teaching experiment. From this analysis I developed a design heuristic for making sense of student mathematical activity considering the role of multiple co-emerging models. To address the third question, I introduced a design heuristic for problematizing 
Investigating Alternate Numeration Systems

mathematical contexts, situating this within the design theory of Realistic Mathematics Education (Gravemeijer, 1998). Alternate bases and non-Euclidean geometry are discussed as two examples of this design heuristic. 
Investigating Alternate Numeration Systems

\section{Chapter 2 : Literature Review}

Chapter 2 is split into two major sections, with the first section providing background literature about teacher knowledge, number and operation, and alternate bases. In the second section of Chapter 2, I discuss the theoretical perspectives which influenced my design experiment.

\section{Structure of the Literature Review}

The review of the background literature is split into three main sections, with the first section focusing on constructs for teacher knowledge. The second section provides an overview of research on whole number and operations, with a particular focus on multidigit multiplication. The split between whole numbers and operation with whole numbers is somewhat artificial, as the study of one generally involves the study of the other. Nevertheless, the review presents whole numbers first to allow a focus on frameworks for understanding the structure of base ten prior to focusing on operations in base ten. The third section of the background literature focuses on prior research using alternate numeration systems with preservice teachers.

Within the sections on number and operation I examine prior research both for children and preservice teachers, providing comparisons of the research within each topic area. Within the literature review I provide key background information about current research in the topics of number and operation for both children and preservice teachers and to situate my research within the wider body of research on number and operation. I place a particular emphasis on the development of multidigit operations, especially multiplication. A special focus is given to sets of studies that present similar research for both children and preservice teachers, such as Fuson's (1997) and Thanheiser's (2009) 
conceptions of multidigit numbers and the digit correspondence task (Kamii, 2000; Ross, 2001).

\section{Teacher Knowledge}

That the quality of mathematics teaching depends on teachers' knowledge of the content should not be a surprise. Equally unsurprising is that many U.S. teachers lack sound mathematical understanding and skill. This is to be expected because most teachers - like most other adults in this country - are graduates of the very system that we seek to improve. (Ball et al., 2005, p. 14)

\section{Mathematical Knowledge for Teaching (MKT)}

In his oft cited 1986 paper, Shulman describes the professional knowledge of teachers and calls for research on this specialized knowledge. He distinguishes three types of knowledge. The first knowledge type is content knowledge which includes knowledge of substantive structures (facts and basic knowledge) and syntactic knowledge (how to make arguments and establish validity within a discipline). Shulman's second knowledge type is pedagogical content knowledge which is knowledge that is specialized to teaching, such as knowledge of where students struggle, preconceptions, and useful representations. The third knowledge type is curricular knowledge, which includes knowledge of the available instructional materials in a particular domain, as well as knowledge of curriculum that will be taught later (vertical) and knowledge of curriculum students will engage with in other subject areas (lateral).

Shulman's call for further research on teacher knowledge was answered by many mathematics education researchers, including Ma (1999); Ball, Hill, and Bass (2005); Ball, Thames, and Phelps (2008); Hill, Ball and Schillig (2008); and Silverman and Thompson (2008). Ma developed the construct of Profound Understanding of 
Investigating Alternate Numeration Systems

Fundamental Mathematics (PUFM) to capture mathematical knowledge that is deep,

broad, and thorough. Ma found that mathematics teachers in the U.S. tend to lack PUFM, resulting in teachers providing explanations that focus on how to do procedures rather than why those procedures makes sense (1999).

Ball et al. (2005) use the term mathematical knowledge for teaching (MKT) to refer to the professional knowledge related to teaching mathematics. In a study comparing the MKT for 700 teachers to the gain scores of their nearly 3000 students on the Terra Nova standardized test, Ball et al. (2005) found that teachers' MKT scores significantly predicted student gain scores. For example, students of a teacher in the top quartile had gain scores that equated to 2 or 3 extra weeks of instruction, compared to students of teachers with average scores. The effect size for the MKT of the teacher was comparable to the effect size for socioeconomic status for the students in the study. One key aspect of this study was the use of a specialized test to measure MKT, rather than a proxy value like the number of prior mathematics courses taken by the teacher.

Ball et al. (2008) developed a domain map for MKT, explaining that their work can be seen as a refinement of Shulman's three categories of content knowledge, pedagogical content knowledge, and curricular knowledge. Ball et al. refer to content knowledge as subject matter knowledge, which they partitioned into the following three categories (see Figure 2-1):

- Common content knowledge refers to knowledge that is not specific to teaching, but used in the same manner in many other settings as well. Teachers should be competent with the content they intend to teach to their students. Ball et al. provide examples of common content knowledge such as 
Investigating Alternate Numeration Systems

knowing that $0 / 7$ is 0 and being able to find a number between two given decimal numbers.

- Specialized content knowledge is the content knowledge that is specific to teaching, such as providing explanations for mathematical procedures, unpacking nonstandard algorithms, and using appropriate representations. Ball et al. distinguish specialized content knowledge from the conceptual understanding of content that they would want all students to have. They differentiate that they "do not hold as a goal that every learner should be able to select examples with pedagogically strategic intent, to identify and distinguish the complete range of different situations modeled by $38 \div 4$, or to analyze common errors" (2008, p. 401).

- Horizon content knowledge refers to knowledge of how a mathematical topic develops across the grade spans. For example, a fourth grade teacher should be aware of how the area model they use for multidigit multiplication relates the multiplication of fractions and equations, as well as relating to division strategies.

Ball et al. rename Shulman's curricular knowledge as knowledge of content and curriculum and place it within the larger category of pedagogical content knowledge. The other two divisions of pedagogical content knowledge are knowledge of content and students, which is the type of knowledge that allows teachers to anticipate student thinking and potential student struggles, and knowledge of content and teaching, which includes knowledge of useful examples, models, and representations for teaching particular content. 


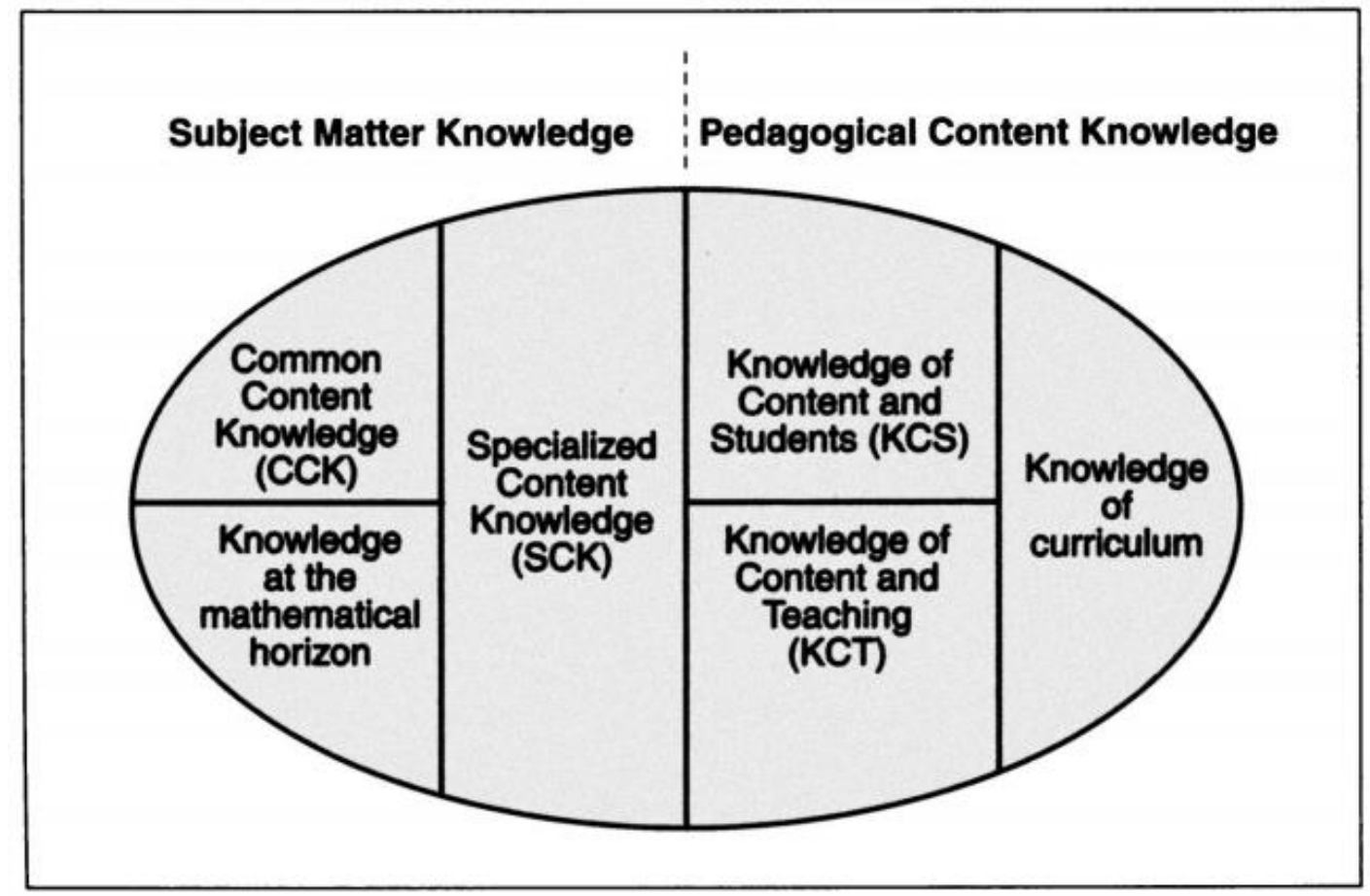

Figure 2-1. A domain map for mathematical knowledge for teaching. Image from Hill, Ball, \& Schillig (2008, p.377).

Hill, Umland, Litke, and Kapitula (2012) link MKT and the mathematical quality of instruction (MQI) made available to students. They used an MQI framework to analyze videos of classroom lessons, focusing on the components of richness of mathematics, working with students and mathematics, errors and imprecisions, and student meaning-making. Hill et al. (2012) found that teachers with high scores on an MKT written assessment had higher levels of MQI, while teachers with low MKT scores also had lower levels of MQI. This research highlights the impact of teachers' MKT on the types of learning opportunities made available to children. 


\section{A Strategy for Supporting MKT: Problematized Mathematical Contexts}

One strategy for supporting the development of MKT is the use of problematized mathematical contexts. I introduce the term problematizing mathematical contexts ${ }^{2}$ to describe the task design heuristic of shifting from routine mathematical context to a novel or non-routine mathematical context. The non-routine context is selected for the pedagogical purpose of developing a stronger understanding of a more routine context. The non-routine context should be similar enough in structure to provide a worthy comparison with the routine context. For example, the taxi-cab metric may be used in a geometry course to provide a non-routine metric to compare against the overly-routine Euclidean metric. Likewise, spherical geometry can be leveraged as a problematized alternative to planar geometry. One hallmark of a problematized mathematical context is that it is introduced with the intention of better understanding a common mathematical context as an example of a wider phenomenon. The goal of Chapter 6 (Problematizing Paper) is to establish problematizing a mathematical context as a potential task design heuristic and to situate this design heuristic within existing theory on task design, particularly within didactic phenomenology.

Alternate bases serve as a problematized mathematical context for PST courses in number and operation (e.g. Bassarear, 2012; Bennett et al., 2012). The alternate base serves to highlight the role of place value and regrouping by altering the values of the places (no longer tens, hundreds, etc.) and the quantity needed to regroup. By investigating alternate bases, PSTs have the opportunity to see base ten as an example of

\footnotetext{
${ }^{2}$ In a search of the literature, I was unable to uncover a definition for the phrase problematized mathematical context in mathematics education research. Therefore this is my own definition. My goal with this phrase is to call attention to this idea as a design heuristic and to suggest future avenues of research related to problematizing mathematical contexts.
} 
Investigating Alternate Numeration Systems

the more general structure of place value number systems (Vygotsky, 1962). Alternate base studies have been used to point to PST misconceptions (Khoury \& Zazkis, 1994; Thanheiser \& Rhoads, 2009; Zazkis \& Khoury, 1993) and to provide strategies for improving knowledge (McClain, 2003; Roy, 2014; Thanheiser, 2014; Yackel et al., 2007). Both roles are discussed further in the section on alternate bases research literature.

\section{Procedural and Conceptual Understanding}

In the previous section I used the phrases "stronger understanding" and "better understanding." Mathematics educators have developed a variety of constructs to capture different types or levels of understanding that students could have for a particular idea. For example, students might be able to perform long division and find correct answers, but they may be unable to explain why the process works or relate the process to a model for division. Skemp refers to "rules without reasons" as instrumental understanding and "knowing both what to do and why" as relational understanding $(1978$, p. 2). Hiebert and Lefvre (1986) use the dichotomy of procedural vs. conceptual knowledge to distinguish two types of knowledge commonly seen in the math classroom. Procedural knowledge refers to knowledge of symbols, rules, and algorithms. Conceptual knowledge is knowledge where, "the linking relationships are as prominent as the pieces of information" (Hiebert \& Lefevre, 1986, pp. 3-4)..

In this study I use Hiebert and Lefve's dichotomy, as well as Lampert's four types of knowledge (intuitive, computational, concrete, and principled) which are described later in the chapter, as they relate to children's understanding of multiplication. PSTs tend to have procedural knowledge of elementary mathematics, but often lack conceptual 
Investigating Alternate Numeration Systems

knowledge, viewing mathematics as a disconnected set of rules (Ball, 1990a; Graeber, 1999; J.-J. Lo et al., 2008; Ma, 1999). In order to build MKT, PSTs must develop a conceptual understanding of the mathematics they will teach.

\section{Understanding the Structure of Base Ten}

In the prior sections I discussed the importance of MKT. In my study, I focus more narrowly on building MKT in the domain of whole number and operation, with a particular focus on multiplication. In order to support PSTs in building MKT in their preservice content courses, it is vital that teacher educators be aware of the types of knowledge PSTs bring to these courses. In the following sections I focus on the content area of whole number and operations, reviewing research about both children's and PSTs' knowledge of base ten and operations within base ten. In this section I begin by discussing the mathematical structure of the base ten number system. This will be followed by a discussion of research on children's and PSTs' understanding of whole numbers in base ten.

\section{Structure of Base Ten}

The base ten number system is a positional number system or place value number system. That is, the value of each digit depends on its location within a number. Consider the example of 282. The 2 on the right is worth 2 ones, while the 2 on the left indicates 2 hundreds, or 200 ones. Each digit in a multidigit number has both a face value (the name of the digit, such as "two") and a place value (such as ones or hundreds). Both the face value and place value must be combined in order to determine the value of a particular number. For example, the number 345 represents 3 hundreds plus 4 tens plus 5 ones. 
The base ten number system represents a powerful and efficient number system, particularly when compared with historic number systems like the ancient Egyptian numeration system. The Egyptian system, like our modern base ten system, relied on powers of ten for the underlying structure. For each power of ten, there was a specific symbol to represent that quantity (see Figure 2-2a). Therefore a number like 345 would be written by making the appropriate number of copies of the symbols for hundreds, tens, and ones (see Figure 2-2b). The Egyptian number system is considered to be an additive number system because the value of a number is found by adding the value of each symbol. To better understand the efficiency of our place value system, imagine writing 987,654 in the Egyptian system. What can be written with just 6 symbols in base ten takes 39 symbols in the Egyptian system.

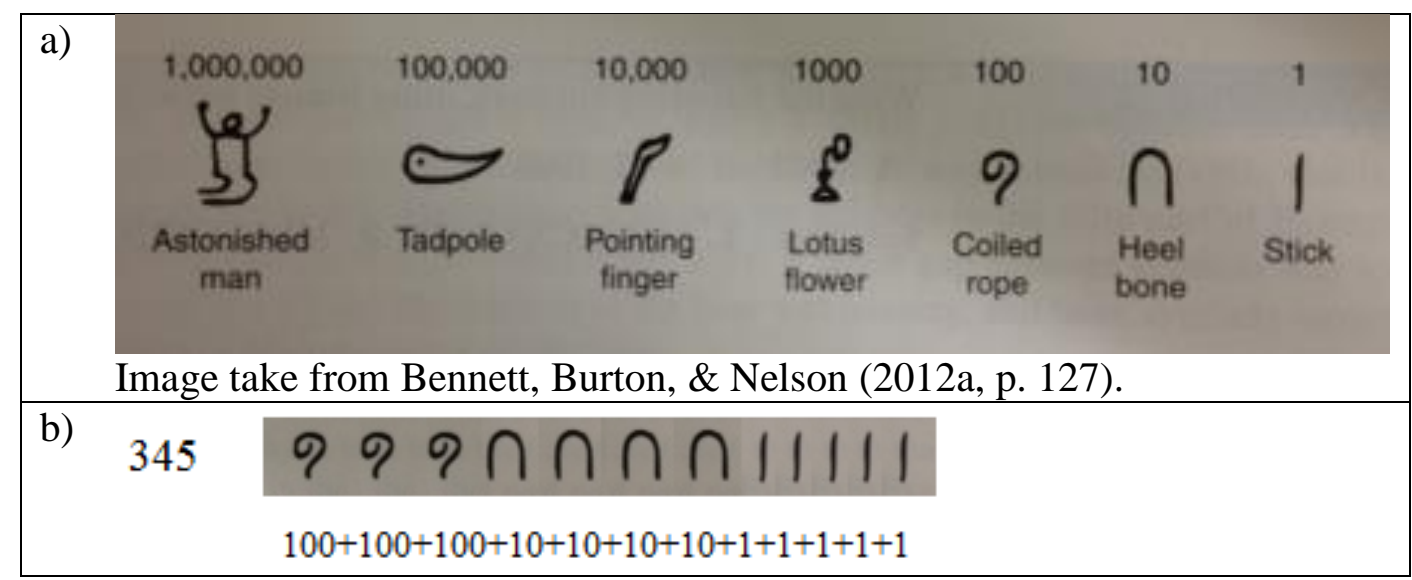

Figure 2-2. The Ancient Egyptian numeration system is an additive numeration system where symbols indicate their value regardless of their location in a number

While base ten is certainly more efficient than the Egyptian system, this same efficiency can make base ten challenging to learn. A student must be able to coordinate multiple types of units (ones, tens, hundreds, etc.) which are denoted with the same set of symbols $(0,1,2,3, \ldots, 9)$. When a student is able to make sense of and use the structure 
of base ten they have developed place value understanding. In the following section I present how the ideas of base ten numbers and operations are discussed within the Common Core State Standards (CCSS), building up from representing two digit numbers in first grade to developing strategies for multidigit multiplication and division by the end of elementary school (National Governors Association Center for Best Practices \& Council of Chief State School Officers (CCSS), 2010).

\section{Common Core Expectations for Number \& Operations in Base Ten}

Number. Within the Common Core State Standards (CCSS, 2010) the development of conceptual understanding of number and operation, both single and multidigit, is captured in the strand Number and Operations in Base Ten (NBT). Beginning in first grade, students lay a foundation for understanding place value by examining the relationships between tens and ones within two digit numbers (1.NBT.2) ${ }^{3}$. A goal of second grade is moving from two to three digit numbers, including the idea that one hundred can be viewed as ten tens (2.NBT.1a). By fourth grade, this reasoning extends to recognizing that in a multidigit number, each digit represents ten times more than the digit in the next column to the right (4.NBT.1).

Operation. Understanding of numbers does not stand alone, but is woven in with operations throughout the Common Core (Fuson \& Beckmann, 2012). Students are expected to use "strategies based on place value, properties of operations, and/or relationships between [operations]" (CCSS, 2010, p. 19). This language about sensemaking strategies is used repeated throughout the Common Core. The Common Core

\footnotetext{
${ }^{3}$ This notation indicates specific CCSS standards, where the first number refers to grade, the acronym refers to the content strand, and the number after the acronym refers to a particular grade level standard within that strand.
} 
distinguishes strategies from standard algorithms, indicating that strategies should be based on student sense-making related to the structure of the number system as well as the properties of operations, such as the commutative, associative, and distributive properties. The NBT Progressions for the Common Core states that " $[\mathrm{u}] \mathrm{se}$ of the standard algorithms can be viewed as the culmination of a long progression of reasoning about quantities, the base-ten system, and the properties of operations" (Common Core Writing Team, 2011, p. 3). Some of the research discussed below questions this role of the standard algorithm as the final goal for student understanding (Carraher, Carraher, \& Schliemann, 1985; Ebby, 2005; Kamii \& Dominick, 1997).

Multiplication. Multidigit multiplication is a central focus of grade 4 within the Common Core. Students are expected to use their models for multiplication, including equal-sized groups (repeated addition) and area/array models, as well as properties of operations, especially the distributive property, to "develop, discuss, and use efficient, accurate, and generalizable methods to compute products of multi-digit whole numbers" (CCSS, 2010, p. 27). In the section on children's understanding of operations and algorithms I review literature that indicates what this student invention process might look like for multiplication (Ambrose et al., 2003; Iszak, 2004). I also discuss research about what specialized knowledge teachers would need in order to support the students as they develop multiplication strategies.

\section{Research on Children's Understanding of the Structure of Base Ten}

When children learn to count, numbers are viewed as collections of ones. For example, 9 refers to 9 ones and the number after 9 is simply a collection of ones that happens to be one larger, rather than a regrouped quantity of one ten (10). Children who 
Investigating Alternate Numeration Systems

are able to count to 15 may not see the 15 as composed of 1 ten and 5 ones (Kamii, 2000). Rather, the 15 may be viewed simply as 15 ones. An important shift in understanding of number occurs when a child is able to repackage a collection of ones as a single, iterable quantity (Fuson et al., 1997; Steffe \& Cobb, 1988). Rather than this shift being a single jump from not seeing tens to seeing and using tens efficiently, research has shown that children build several distinct conceptions as they construct meaning for multidigit numbers (Fuson et al., 1997; Steffe \& Cobb, 1988).

Fuson et al. (1997) identify six different conceptions (five correct and one incorrect) that children may hold for two-digit numbers. The five correct conceptions are referred to as the UDSSI model, referring to the five names unitary, decade, sequence, separate, and integrated. Students may hold multiple correct conceptions at once, as well as the incorrect concatenated digits conception. These conceptions are briefly illustrated in Figure 2-3 (Images adapted from Fuson et al. 1997, p.139). Each conception relates quantities, symbols, and number words. In the example of 53, the 5 could be conceived of as 5 units (incorrect concatenated digits) or as 50 of the dots in a cloud of 53 (unitary multidigit) or as a cloud of 50 dots (decades and ones) separated from the other 3 dots. More sophisticated understandings treat the 5 as grouped into 5 sets of ten, where the ten can be a loose collection of ten units (sequence tens and ones) or a unit of size ten (separate tens and ones), or both flexibly (integrated sequence-separate tens and ones). These six categories extend to whole numbers larger than two digits. 


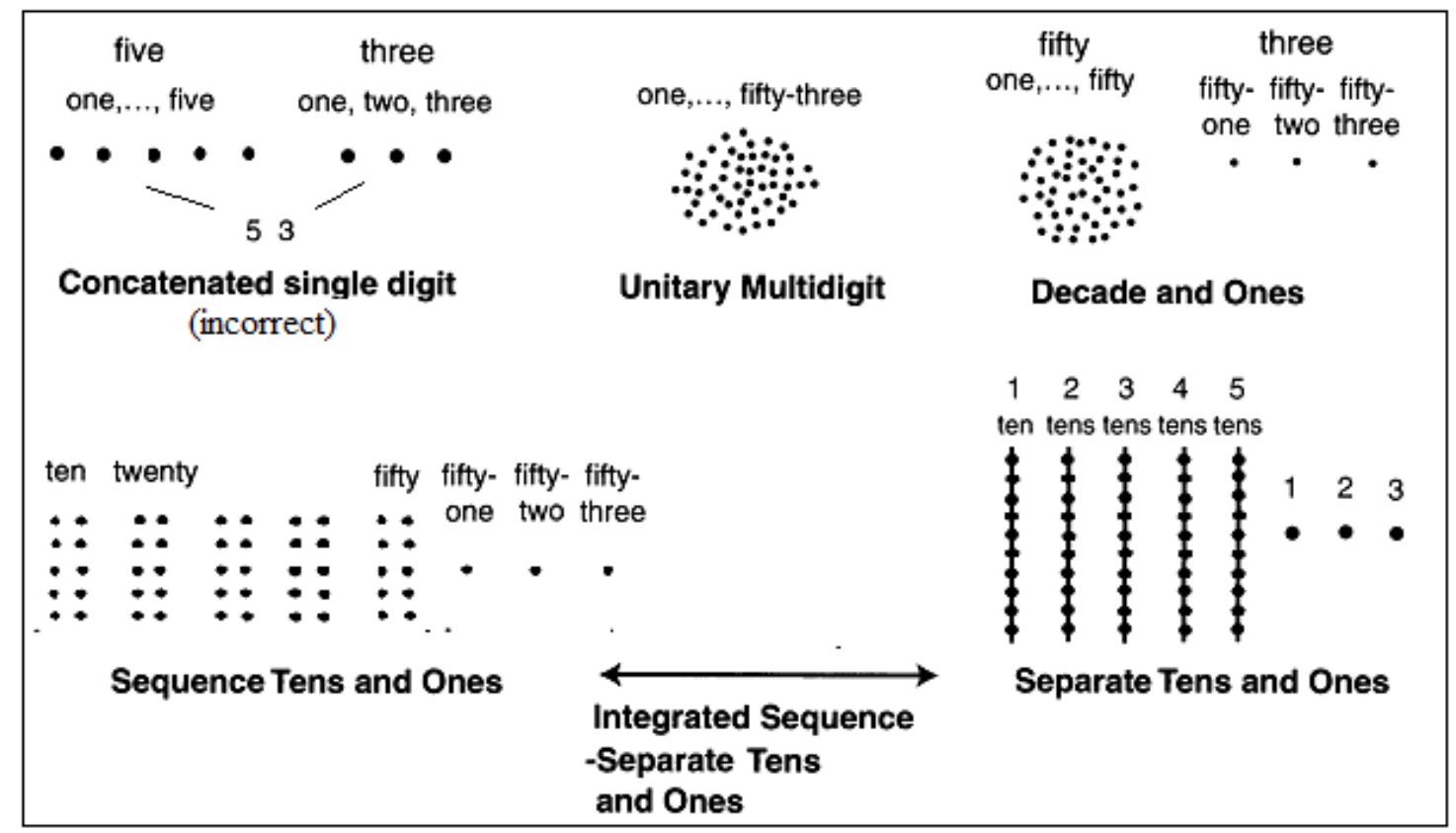

Figure 2-3. Six different conceptions for the number 53, with the conceptions in the bottom row highlighting subsets of ten within the total of 53. (Image adapted from Fuson et al. 1997, p. 139)

Kamii warns that "[t]here is a world of difference between being able to think only successively about "one ten" and "ten ones" and being able to think about both simultaneously" (2000, p. 31). Kamii (1994) describes a hierarchical structure for conceptualizing base ten numbers in which a system of tens in built on top of a system of ones without losing the underlying system of ones. Likewise, a system of hundreds can be built atop the tens and ones structure without losing the prior structure. An image of this hierarchical structure appears in Figure 2-4.

The types of understandings students hold about multidigit numbers affect their solution strategies for multidigit operations. Student strategies for operations develop alongside their developing conceptions of multidigit numbers, as indicated by the CCSS (2010). Frequently errors in solution strategies can be linked to a student's conception of 
numbers as concatenated digits (Fuson, 1990; Fuson et al., 1997; Verschaffel et al., 2007).

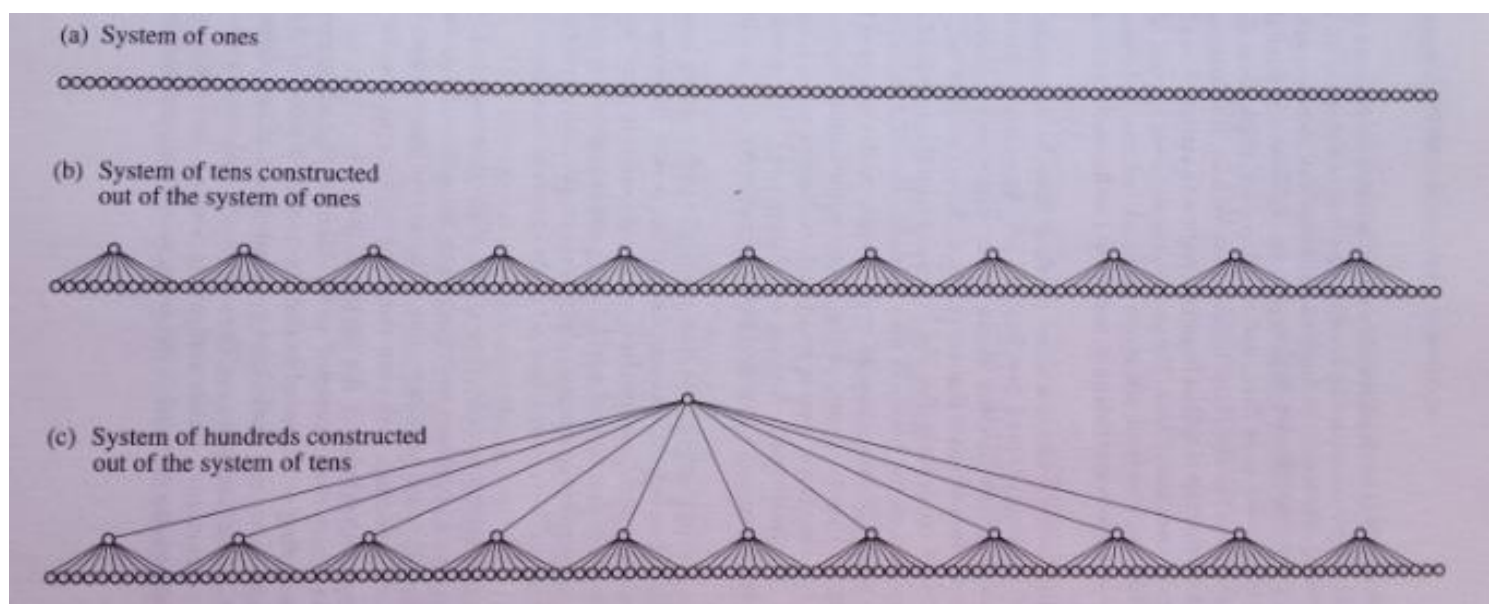

Figure 2-4. Larger units are built out of smaller units without losing the underlying structure of the smaller units (image taken from Kamii, 1994, p.10).

\section{Research on preservice teachers' understanding of the structure of base ten}

Thanheiser (2009a), noting a lack of research on PSTs understanding of multidigit numbers, developed a framework similar to Fuson et al. (1997). Thanheiser's framework was based on her interviews with PSTs as they solved and explained multidigit addition and subtraction problems. Thanheiser's interviews revealed four distinct conceptions with two (incorrect) conceptions based upon concatenated digits. The lowest category was concatenated digits only, in which PSTs would interpret 234 as 2 ones, 3 ones, and 4 ones. The category of concatenated digits plus captures conceptions with at least one unit type incorrect, such as interpreting 234 as 200 ones, 3 ones, and 4 ones. A groups of ones conception implies that each digit is thought of as the appropriate numbers of ones (i.e. 234 is 200 ones, 30 ones, and 4 ones), but that the PST does not flexibly repackage ones using reference units. A reference units conception is when the 200 in 234 can be thought of as 2 hundreds, 20 tens, or 200 ones. In her study of 15 PSTs, Thanheiser 
found that ten of the PSTs held one of the incorrect conceptions, while only three held the most advanced conception of reference units, prior to instruction.

Preservice (and in-service) teachers may conceal their limited conceptions of multidigit numbers by using the language of place value procedurally (Ball, 1988; Ma, 1999; Thanheiser, 2009a; Thanheiser et al., 2013). For example, PSTs may be able to label the columns of a three digit number as units, tens, and hundreds, but continue to treat the numbers as concatenated single digits. PSTs and teachers with a limited conception of multidigit numbers struggle to explain the mathematics embedded in standard algorithms (Ball et al., 2005; Ma, 1999; Thanheiser, 2009a, 2012), such as the meaning of the popular terms "carrying," "borrowing," and "regrouping." Simply informing PSTs about the 10 to 1 relationship in place values is not sufficient to build conceptual understanding of the structure of base ten (Thanheiser, 2009b).

\section{Comparing the Frameworks for Children and PSTs}

While Fuson et al.’s (1997) UDSSI framework (not including concatenated digits) represents a collection of correct conceptions that children may progress through, Thanheiser's framework is intended to capture the types of knowledge PSTs bring to their coursework. Thanheiser's framework contracts Fuson's five correct categories to two, with decades and ones corresponding to groups of ones and integrated sequenceseparate tens and ones corresponding to reference units. Meanwhile, Thanheiser's framework expands the conception of concatenated digits to two categories.

An additional tool to assess and compare children's and PSTs' understanding of the structure of the base ten system is a brief task referred to as the digits correspondence task (Ross, 2001). The digits correspondence task involves representing a number, such 
as 25 , with 25 randomly arranged objects, such as pennies. Referencing the written number " 25 " the researcher poses two questions, asking what each digit represents. First the researcher circles the 5 in 25 and asks "What does this part of your 25 have to do with how many pennies are in the picture?" Next the researcher circles the 2 and asks "How about this part? What does THIS part have to do with how many pennies are in the picture?" (Ross, 2001, p. 897). A correct response links 5 pennies with the 5 and 20 pennies or 2 groups of 10 pennies with the digit 2 . Ross (2001) used the digits correspondence task for 85 PSTs in their first term math content course. Ross found that only $53 \%$ of PSTs were successful, which is a similar success rate to fifth graders. In a similar study by Kamii, when fourth graders were asked what the "1" in " 16 " referred to, only 51\% recognized the "1" as ten (Kamii, 2000). Children and PSTs often focused on the face value of a number rather than the place value.

In the next section I shift from discussing whole numbers to discussing operations with whole numbers.

\section{Understanding Operations \& Algorithms in Base Ten}

Standard algorithms for whole number operations are both efficient and dense.

Standard algorithms have evolved over generations to become compact and removed from their conceptual basis (Ambrose et al., 2003; Fuson, 2003; Verschaffel et al., 2007). While this compactness lends to efficiency in execution of standard algorithms, it hinders students developing conceptual understanding of the algorithms (Thompson, 2010). Verschaffel et al. note that "standard practice in the use of algorithms even demands that one does not think about what the digits represent if one does not want to become confused" (2007, p. 574). The recent reform movements in K-12 mathematics, embodied 
in the NCTM Standards (National Council of Teachers of Mathematics, 2000) and then in the Common Core State Standards (National Governors Association Center for Best Practices \& Council of Chief State School Officers, 2010), indicate that children should have a conceptual understanding of the algorithms they use, rather than simply speed and accuracy.

\section{Research on Children's Understanding of Multidigit Operations / Algorithms}

First I focus on research that indicates effects of learning algorithms without conceptual basis. Then I discuss a few studies which point to conceptual approaches to learning, particularly with the instructional design heuristic of guided reinvention.

Learning pre-ordained algorithms without understanding may be damaging to children's intellectual curiosity. Kamii and Dominick argue that "Algorithms are harmful to children's development of numerical reasoning for two reasons: (a) They 'unteach' place value and discourage children from developing number sense, and (b) they force children to give up their own thinking" (1997, p. 58). For example, children are inclined to think of numbers from left to right, dealing with larger digits first, but most algorithms move from right to left and treat each digit as if it represented ones. In a study comparing the performances of students who had been taught algorithms with students who had not, the "no algorithms" students arrived at the correct answer more often than the algorithm based students and in the case of error, their wrong answers were more likely to be "close" to the right answer (Kamii \& Dominick, 1997). In addition to being more likely to get the correct answer, the "no algorithms" students were more likely to be able to explain why their strategies made sense. These results are consistent with a study by Carroll (1999) indicating that students using non-standardized strategies were more able 
to justify their methods. While the Common Core indicates that knowledge of the standard algorithms is the final goal of student invented strategies, Kamii and Dominick (1997) argue that building number sense is the final goal and that the conventional algorithms should not be taught. Likewise, Carraher et al. (1985) warns against placing school algorithms on a pedestal while ignoring student invented solution strategies.

Ebby (2005) uses a longitudinal study to illustrate the problems Kamii and Dominick (1997) identified, following one student from second to fourth grade. Ebby found that the introduction of standard algorithms hindered the student's development of place value understanding by providing her with solution strategies that could be executed without understanding of the ten to one relationship between places. In addition, Ebby argues that learning the algorithms without understanding created a view toward mathematics as an arbitrary set of rules and placed the authority for correctness within the teacher and textbook rather than within a student's own sense-making. Pesek and Kirshner (2000) found that teaching procedural skills prior to conceptual knowledge caused cognitive and attitudinal interference, leading students who had learned procedures first to make errors that were not made by students who had learned concepts first.

Given that learning standard algorithms without understanding is potentially damaging to students (Kamii \& Dominick, 1997), a possible solution is to provide students with an opportunity to invent efficient algorithms based on place value understanding. Realistic Mathematics Education (RME) (Gravemeijer \& Cobb, 2006) is an instructional design theory that includes guided reinvention as a core design heuristic. In RME, students are given contextually real problems which encourage student use of 
intuitive models to create solution strategies. Prior examples of RME research around multidigit arithmetic include a study using the empty number line for addition and subtraction (Klein, Beishuizen, \& Treffers, 1998), studies of developing multiplication strategies (Armanto, 2008; Treffers, 1987a), and studies of progressive mathematization with long division (Treffers, 1987a; van Putten et al., 2005). In all of these studies, students move from intuitive to more efficient strategies by solving problems and sharing solution methods. Rather than a view of mathematics which puts the teacher or textbook as authority, RME places the authority on sense-making and student strategies.

Rather than focusing on all four operations in base ten, I have decided to narrow my focus to multiplication. I have purposefully selected multidigit multiplication in order to focus attention on the multiplicative structure of our place value number system, where each place value is a multiple of the place value to its right. Efficient and sophisticated strategies for multidigit multiplication, including the standard U.S. algorithm, make use of this structure. Prior to discussing the research on children's understanding of multiplication, I highlight some key mathematical ideas in multidigit multiplication.

\section{Key Ideas and Models in Multidigit Multiplication}

There are certain to be many interpretations on what it means to "understand multidigit multiplication," from the limited perspective of proficient use of the U.S. standard algorithm to more broad conceptual perspectives. Rather than claiming to definitively answer the question of what it means to understand multidigit multiplication, I limit myself to explaining some key ideas of multidigit multiplication and relating those ideas to the Common Core (CCSS, 2010). 
According to the Common Core, students should be able to use models such as repeated addition and area/array to develop strategies for solving multidigit problems. Consider the example of $14 \times 23$ below. The problem $14 \times 23$ could be considered as (a) 14 groups of size 23 (repeated addition / equal groups model) (Figure 2-5a) or as (b) a rectangular array with dimensions of 14 × 23 (area / array model) (Figure 2-5b). Prior research indicates that children and PSTs tend to rely on the primitive model of repeated addition (Fischbein, Deri, Nello, \& Marino, 1985; Graeber, Tirosh, \& Glover, 1989) rather than area, though the two models can be tied together. In particular, one could image rearranging the 14 groups as 14 rows to visualize the area model.

\begin{tabular}{|c|c|}
\hline 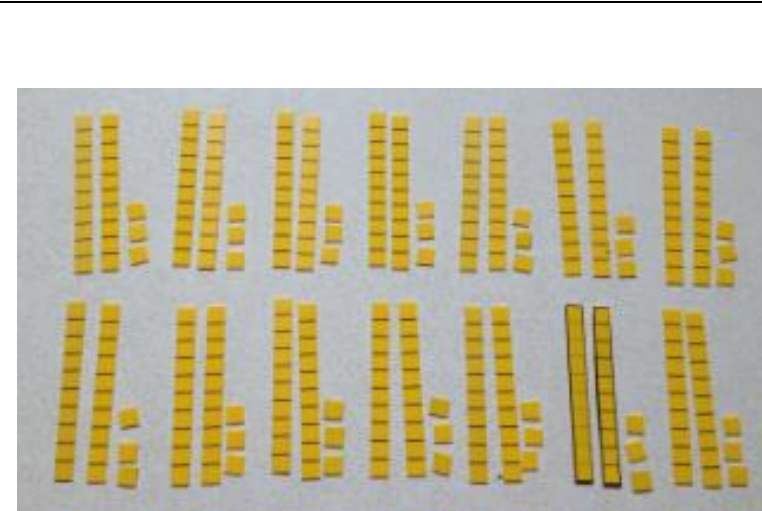 & 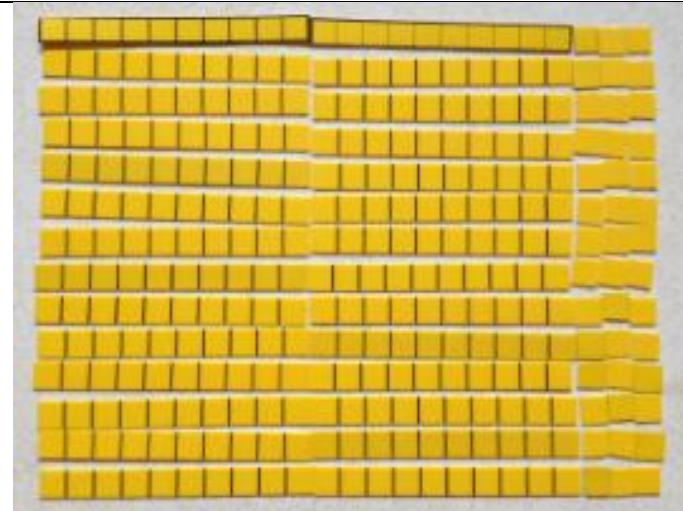 \\
\hline $\begin{array}{l}\text { a) } 14 \times 23 \text { can be considered as } 14 \text { groups of } \\
\text { size } 23 \text {. This model is referred to as the } \\
\text { repeated addition or equal groups model. }\end{array}$ & $\begin{array}{l}\text { b) } 14 \times 23 \text { is arranged as a rectangular } \\
\text { array with } 14 \text { rows of width } 23 \text {. This } \\
\text { model is referred to as the area or array } \\
\text { model. }\end{array}$ \\
\hline
\end{tabular}

Figure 2-5. The repeated addition and area models are represented visually.

Under either model, it can be convenient to split one or both of the numbers using the distributive property. For example, Figure 2-5(a) could be split into 10 groups of 23 and 4 groups of 23 . Then each of these easier sub-problems could be solved separately and rejoined to find the total. Likewise, within the area model in Figure 2-5b either 14 or 23 or both could be split to make the problem into a series of easier sub-problems. Figure 
Investigating Alternate Numeration Systems

2-6a demonstrates the area model split into $10 \times 23$ and $4 \times 23$. Figure $2-6 \mathrm{~b}$ shows the area model split into 4 sub-areas. When a multiplication problem is split into subproblems, this is referred to as the partial products strategy for multiplication.

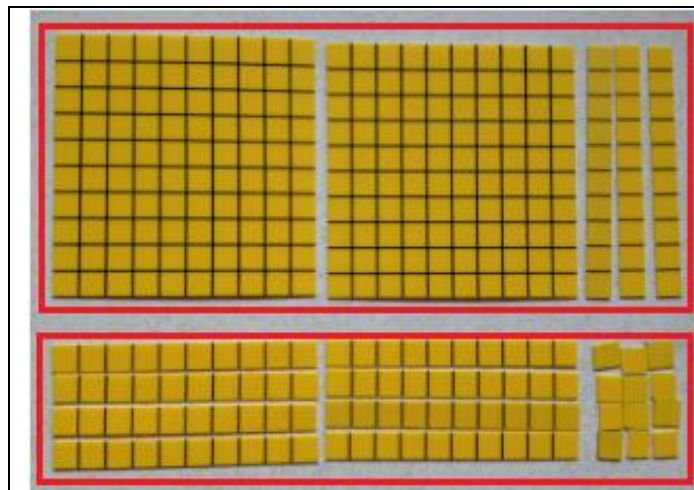

a) The 14 rows of 23 are split into 10 rows of 23 and 4 rows of 23 .

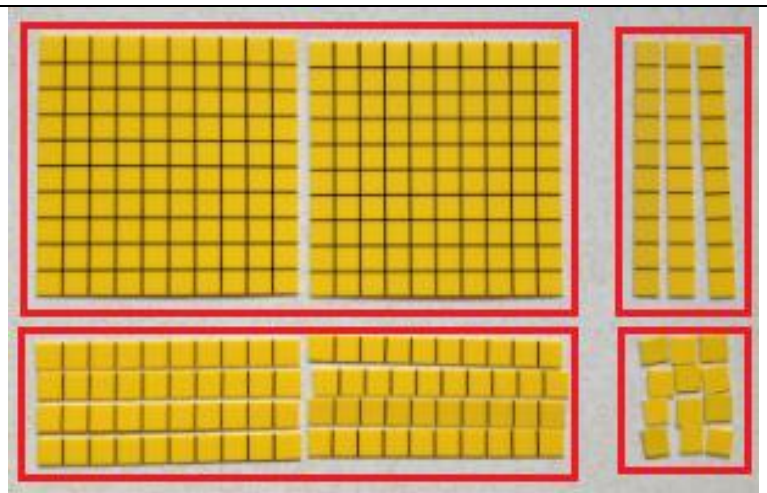

b) Both the 14 and 23 are split along place value lines to create 4 sub-areas with dimensions $10 \times 20,10 \times 3,4 \times 20$, and $4 \times 3$.

Figure 2-6. The area model is split into sub-areas which are easier to calculate.

Students may develop an understanding of multidigit multiplication by exploring the repeated addition and area models with particular numbers, developing and sharing strategies which become more efficient and more general. The standard U.S. algorithm for multidigit multiplication leverages the same partial products strategy illustrated above. In Figure 2-7, I provide an example of the standard U.S. algorithm for 14 x 23, unpacking the links between the written algorithm and the underlying partial products strategy. 


\begin{tabular}{|c|c|c|c|c|}
\hline $\begin{array}{l}\text { a) } 4 \text { × } 3=12 . \text { The } 12 \text { is } \\
\text { split into } 1 \text { ten and } 2 \text { ones, } \\
\text { with the } 2 \text { ones recorded } \\
\text { in the ones column and the } \\
1 \text { ten regrouped and noted } \\
\text { above the } 2 \text { in } 23 \text {. }\end{array}$ & $\begin{array}{r}23 \\
\times \quad 14 \\
2\end{array}$ & \multicolumn{2}{|c|}{$\begin{array}{l}\text { b) } 4 \times 2 \text { tens }=8 \text { tens, } \\
\text { plus } 1 \text { more ten from the } \\
\text { prior step. The } 9 \text { tens } \\
\text { are recorded in the tens } \\
\text { column. }\end{array}$} & $\begin{array}{r}1 \\
\times \quad 3 \\
\times 14 \\
92\end{array}$ \\
\hline \multicolumn{2}{|c|}{$\begin{array}{l}\text { These first } 2 \text { steps combine to form the } \\
\text { partial product of } 4 \times 23=92 \text {. }\end{array}$} & 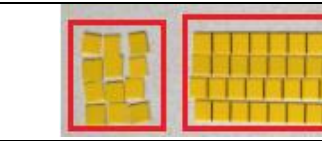 & $\begin{array}{ll}71 \\
11 \\
11 \\
11\end{array}$ & 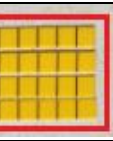 \\
\hline $\begin{array}{l}\text { c) } 1 \text { ten } \times 3=3 \text { tens. The } \\
3 \text { tens are recorded in the } \\
\text { tens column. Often } \\
\text { students record a } 0 \text { in the } \\
\text { ones column, either to } \\
\text { indicate the value of the } 3 \\
\text { as thirty, or to serve as a } \\
\text { mental reminder to shift } \\
\text { the digits to the left. }\end{array}$ & $\begin{array}{r}\frac{1}{23} \\
\times \quad 1 / 4 \\
92 \\
30\end{array}$ & $\begin{array}{l}\text { d) } 1 \text { ten } \times 2 \text { tens }= \\
2 \text { hundreds. The } \\
\text { two hundreds are } \\
\text { recorded in the } \\
\text { hundreds column. }\end{array}$ & $\begin{array}{r}\mathrm{x} L \\
2\end{array}$ & \\
\hline \multicolumn{2}{|c|}{$\begin{array}{l}\text { These two steps combine to form the } \\
\text { partial product of } 10 \times 23=230 .\end{array}$} & 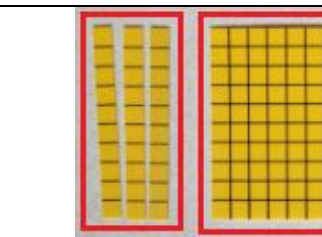 & & 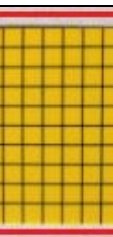 \\
\hline $\begin{array}{l}\text { e) The } 2 \text { partial products, } \\
\text { appropriately aligned by } \\
\text { place value, are summed to } \\
\text { arrive at the total of } 322 \text {. }\end{array}$ & $\begin{array}{r}23 \\
\times \quad 14 \\
92 \\
+230 \\
322\end{array}$ & & & \\
\hline
\end{tabular}

Figure 2-7. The standard U.S. algorithm for multiplication, using the example $14 \mathrm{x} 23$.

Having established some background knowledge and terminology in the domain of multiplication, next I present research on children's understanding of multidigit multiplication.

\section{Research on Children's Understanding of Multidigit Multiplication}

In the Third International Mathematics and Science Study (TIMSS), the following multiple choice problem was posed to third and fourth graders in over 20 countries: "25 x 18 is more than 24 x 18. How much more?" (Mullis et al., 1997, p. 70). Only 30\% of third graders and $45 \%$ of fourth graders selected the correct answer of a difference of 18 . 
Investigating Alternate Numeration Systems

The scores for U.S. students were similar, with $31 \%$ for third graders and $46 \%$ for fourth graders. The answer of a difference of 1 was the most popular distractor, selected by $42 \%$ of third graders and $35 \%$ of fourth graders internationally. While an answer to a multiple choice question does not capture a student's complete conception of the topic, the results for this question indicate a problematic understanding of multiplication for many U.S. students.

What does it mean to understand multidigit multiplication? Lampert (1986) approaches this question with a framework of four types of knowledge about multidigit multiplication, based upon her experiences in a fourth grade classroom. The four types of knowledge are intuitive, computational, concrete, and principled. Intuitive knowledge is situationally based, such as the type of everyday knowledge used outside of school. Computational knowledge (how to solve problems with symbols) and concrete knowledge (how to rearrange manipulatives to solve problems) can be combined to form a procedural category of knowledge. Principled knowledge, referred to as conceptual knowledge by some researchers, relies on such principles as the associative, commutative, and distributive properties, doubling, convenient splittings, place value, and decomposing and recomposing numbers. Lampert advocates developing all four types of knowledge together, rather than focusing on a limiting subset. Lampert's types of knowledge could be applied to any operation as well as contexts beyond whole number calculations.

While Lampert discusses different types of knowledge, Ambrose, Baek, and Carpenter (2003) elaborate on different strategies students might develop as they move from intuitive to more efficient calculation methods for multidigit multiplication. Beginning strategies include direct modeling using counters and move to direct modeling 
with tens and ones. The repeated addition of earlier models is abridged with simple or complex doubling strategies in which students may create compound units, such as 4 books for $\$ 48,8$ books for $\$ 96$, and 16 books for $\$ 192$. The most advanced strategies are when the multiplier or both factors are split along place value. For example, interpreting $14 \times 23$ as ten copies of 23 and then four more copies of 23 involves splitting only the multiplier while a more expanded algorithm may involve splitting both the 14 and 23 into tens and ones. These progressively advancing strategies are consistent with and build upon Treffers' (1987a) research.

While Ambrose, Baek, and Carpenter (2003) and Treffers (1987) do not indicate the role of the area model in student reasoning, Iszak (2004) focuses specifically on the area model for multiplication. By using and discussing strategies based on area contexts, students were able to make sense of the shared strategy of an expanded algorithm based upon the distributive property and partial products. Although the strategies were specified within the curriculum, Iszak advocates class exploration of multiple initial strategies with the intent of encouraging access to all students rather than a more teacher led approach along a specific path.

\section{Research on PSTs' and Teachers' Understanding of Multidigit Operations}

In an earlier section I described why learning algorithms without understanding is damaging to children (Carroll, 1999; Ebby, 2005; Kamii \& Dominick, 1997; Pesek \& Kirshner, 2000). A corollary to this statement is that, in some sense, PSTs may be damaged by their prior mathematical background. Studies show that frequently PSTs and teachers have insufficient mathematical knowledge of the topics they are expected to teach (Ball, 1988; Ma, 1999; Thanheiser, 2009a). For example, PSTs may not see the 
need to go beyond finding the numeric answer to justifying why the strategy works (Graeber, 1999; J.-J. Lo et al., 2008; Philipp et al., 2007) When PSTs are asked to justify why an algorithm or strategy works, they often respond with a restatement of how to perform the algorithm (Ball, 1990a; J.-J. Lo et al., 2008). This indicates that PSTs may be unaware that there is a conceptual underpinning for the algorithm. In her book comparing U.S. and Chinese teachers, Ma states "Mathematical knowledge is based on both convention and logic. However, convention in this case serves as a shelter for those who don't have a conceptual understanding of a mathematical procedure" $(1999$, p. 31).

\section{Research on PSTs' and Teachers' Understanding of Multidigit Multiplication}

The research on PST and teacher understanding of multidigit multiplication tends to focus more heavily on what PSTs know and don't know, rather than how they develop conceptual understanding. Ma's (1999) study of in-service teachers in the U.S. and China revealed that U.S. teachers were proficient in performing multidigit multiplication, but $61 \%$ were unable to provide a conceptually based explanation. The teachers were asked to examine a three digit by three digit multiplication problem with a common student error of incorrectly lining up the partial products (see Figure 2-8). The teachers were asked to reflect on how they would work with students who had made this mistake. Approximately $70 \%$ of U.S. teachers believed the primary issue was a procedural error in carrying out the algorithm, while $30 \%$ recognized that students who performed in this manner did not understand the mathematics embedded in the algorithm. This is consistent with other research on operations which indicates that PSTs or teachers may believe that knowing how to find an answer is equivalent to understanding (Graeber, 1999; J.-J. Lo et al., 2008). Additional research points to the limited and inflexible 
understanding of multidigit multiplication held by PSTs (Graeber et al., 1989; Southwell $\&$ Penglase, 2005).

\begin{tabular}{|c|c|}
\hline $\begin{array}{r}123 \\
\times 645 \\
615 \\
492 \\
738 \\
1845\end{array}$ & $\begin{array}{l}\text { A common student error when multiplying multidigit numbers } \\
\text { is to incorrectly line up the partial products. For example, when } \\
\text { the } 4 \text { (tens) in } 645 \text { is multiplied by } 123 \text {, the result of } 492 \text { refers } \\
\text { to } 492 \text { tens rather than } 492 \text { ones and so it should be recorded } \\
\text { one column to the left of its current arrangement. Alternately } \\
\text { stated, } 4 \text { tens } x 123=4920 \text {. }\end{array}$ \\
\hline
\end{tabular}

Figure 2-8. U.S. teachers focused on the procedural error, rather than the student's underlying misconception (Ma, 1999).

In their 2008 study, Lo, Grant, and Flowers provide insight into the learning challenges faced by PSTs as they attempted to make sense of multidigit multiplication in a course on number and operation. Rather than relying on examples of children's thinking or asking PSTs to abandon their known rules, tasks were designed to "bring out erroneous application of previous learned rules, and thus highlight the need for sound mathematical reasoning and justification" (J.-J. Lo et al., 2008, p. 8). Lo et al. found that PSTs struggled to create reasoning strategies for multiplication. One source of trouble was the belief that finding the numeric answer was sufficient (without justifying the strategy). Another source of the trouble appeared to be a lack of multiplicative structure. For example, in multiplying $17 \times 36$, a PST with a multiplicative structure may see this as 17 rows of 36, while a PST without a multiplicative structure may see it as two distinct quantities: 17 units and 36 units. Once strategies were developed, PSTs struggled to justify these strategies and to link these strategies with visual models. Sometimes visual models were included without explanation and sometimes visual models were added "after the fact" and did not match the strategy actually employed. Consistent with the findings of Graeber, Tirosh, and Glover (1989), Lo et al. found that PSTs appeared to 
hold a preference for the repeated addition model of multiplication rather than the more powerful area model. Similar to the work of Simon and Blume (1994), Lo et al. found that the area model was challenging for PSTs to understand, though it proved useful for some students in decomposing area into smaller regions.

Although not strictly in the domain of base ten whole numbers, a study by Rathouz (2011) provides insight into potential goals and challenges in using the area model for decimal multiplication. In her semester long study with 35 PSTs, Rathouz found that PSTs struggled to coordinate the type of dimension (length or area) with the magnitude of a unit. For example, if the length of a unit square was one tenth by one tenth, PSTs may consider the area to also be one tenth rather than one hundredth. The area model represents a tool which may assist PSTs in generating conceptual explanations about unit coordination. Rathouz states "In this unfamiliar territory of decimal numbers, PSTs confronted concepts of dimensionality, unit labeling, and unit coordination that were not at issue in the domain of whole numbers" $(2011$, p. 10). The difficulties Rathouz uncovered with decimals may be a symptom of more systemic issues with place value understanding. For example, when multiplying 30 by 20, PSTs will likely get 600 , but when asked whether 3 tens $x 2$ tens is 6 tens or 6 of something else, these same PSTs may not be able to create a conceptually sound response.

\section{Comparing Research on PSTs' and Children's Understanding of Whole Number and Operations}

While recent research on children's understanding of whole number and operation focuses on how children might build up their knowledge, the research on PSTs focuses more heavily on what PSTs know and don't know. The reason for this difference may be 
the notion that PSTs should already understand whole numbers. There appears to be limited research on how PSTs can move beyond procedural fluency and develop a conceptual understanding of efficient multidigit algorithms. In a quantitative overview of recent research on PSTs understanding of whole number and operation Thanheiser et al. found that out of 26 studies published between 1978 and 2012 only 2 focused on PSTs development of conceptions (i.e. learning) while the other 24 studies focused on snapshots of PSTs knowledge (2014)

With the intention of adding to this small body of research on PST learning, in my study I build a local instructional theory for PSTs to develop a conceptual understanding of multidigit multiplication. This local instructional theory is built on (a) the prior research on multidigit multiplication and (b) the problematized context of an alternate base. Another two components of my study involved an analysis of how the context of alternate numeration systems is currently leveraged in PST textbooks and a theoretical report which situates alternate bases as an example of a broader design heuristic of problematizing mathematical contexts. The concept of an alternate base is discussed further in the following section.

\section{An Introduction to Alternate Bases}

Prior to delving into the research on alternate bases, I provide examples of alternate base systems. Our base ten number system is built upon powers of ten. The place values in a base ten multidigit number correspond to ones $\left(10^{0}\right)$, tens $\left(10^{1}\right)$, hundreds $\left(10^{2}\right)$ and so on (in addition to negative integer powers to the right of the decimal). As described in Chapter 1, an alternate base number system, also called a nondecimal system, is a number system based on powers of a number other than ten. Base 
five is an alternate base that is common to a number of PST mathematics textbooks (e.g. Bassarear, 2012b; Bennett et al., 2012; Freitag, 2012).

Base five is built on powers of five, with place values corresponding to $5^{0}, 5^{1}, 5^{2}$, $5^{3}$, etc. That is, in base five, there is a ones place, fives place, twenty-fives place, etc., rather than a ones place, tens place, hundreds place, etc. Consider the number $123_{\text {five, }}$ which refers to 1 group of twenty-five, 2 groups of five and 3 ones (see Figure 2-9a). To provide some language to talk about the numbers in base five, a group of five ones will be referred to as a long, a collection of twenty-five ones (or five longs) will be referred to as a flat, and a collection of one hundred and twenty-five ones (or five flats) will be referred to as a long flat (see Figure 2-9b). Each time you collect five of one type of unit, those units can be regrouped into one of the next larger unit. For example, five longs can be regrouped as one flat. The numeral $123_{\text {five }}$ can be read aloud as 1 flat 2 longs 3 ones ${ }^{4}$. This base five language is taken from Bennett, Burton, and Nelson (2012a).

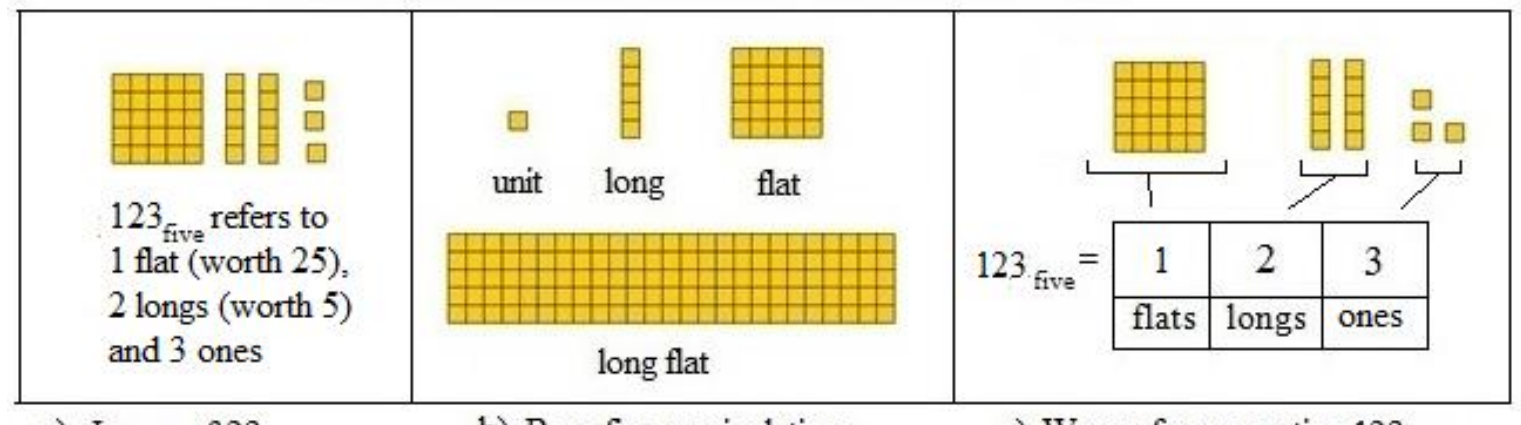

a) Image of $23_{\text {five }}$

b) Base five manipulatives

c) Ways of representing $123_{\text {five }}$

Figure 2-9. An introduction to base five language and imagery.

While base five uses the symbols $0,1,2,3$, and 4 , some alternate bases are presented with historic notation systems. For example, the Mayan number system is an

\footnotetext{
${ }^{4} 123_{\text {five }}$ should not be read aloud as "one hundred twenty three base five", as that confounds base ten and base five language. Base five does not have a hundreds place.
} 
example of base 20. The Mayan system uses dots to represent ones and bars to represents fives. A single place can hold up to 3 bars and 4 dots (representing 19). To represent larger numbers, the Mayan system stacks vertically, with the lowest level indicating groups of one, the next level groups of 20 , and the next level groups of 400 (i.e. $20^{2}$ ). Examples are provided in Figure 2-10. Both the Mayan and Egyptian systems (mentioned in Chapter 1) are used in PST math content textbooks to discuss different types of positional number systems (Bassarear, 2012c; Bennett et al., 2012a).

In addition to historic alternate bases, there are also two alternate bases systems commonly used with computer technology: binary (base 2) and hexadecimal (base 16). The binary system uses only $0 \mathrm{~s}$ and $1 \mathrm{~s}$, because any time there are two of one unit type, those two can be regrouped to the next larger unit type. The ability to represent vast amounts of data with only $0 \mathrm{~s}$ and $1 \mathrm{~s}$ serves as the basis for modern computers.

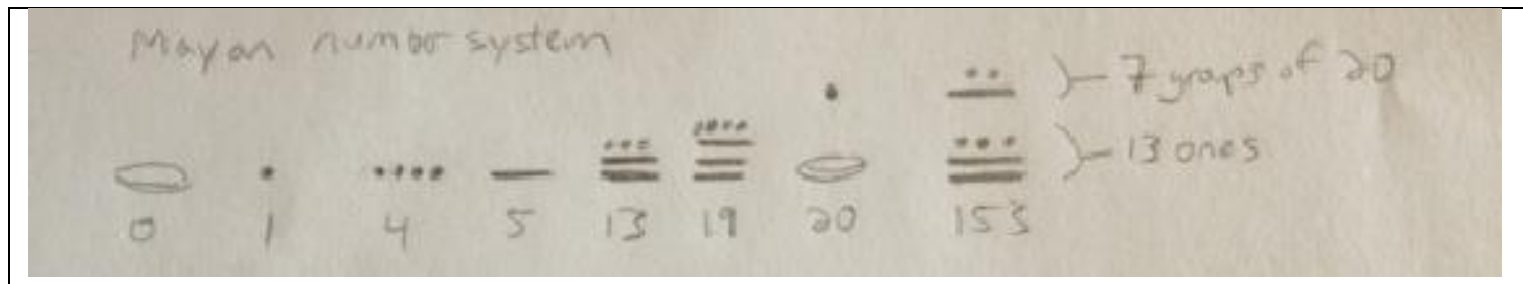

In the Mayan number system, one is recorded as a dot and five is recorded as a bar, while zero is represented with a shell. Each row can represent up to 19 using dots and bars. To represent 20, a dot is placed in the "twenties row," above a shell representing zero ones in the "ones row." For the example of 153, there are seven groups of size 20 and 13 ones, so a dot and two bars are used to represent the seven twenties, which is placed above the 13 ones.

Figure 2-10. The Mayan and base five number systems are two examples of alternate numeration systems (Bennett et al., 2011).

\section{Research on Alternate Bases and Preservice Teachers}

The use of alternate bases as a teaching tool has a history with many names. These names include historic titles such as Mayan and Babylonian; fictional titles like 
Investigating Alternate Numeration Systems

Xmania (Schifter \& Fosnot, 1993), Alphabitia (Bassarear, 2012c), the Land of Eight

(Treffers, 1987b), and Orpda (Hopkins \& Cady, 2007); and mathematical titles, including

alternate bases, non-decimals, base five, sexagesimal (base 60 or clock arithmetic), and binary.

Alternate bases have been used to explore place value understanding, multidigit addition and subtraction of whole numbers, and decimal fraction understanding. I have been unable to unearth any research on the use of alternate bases for teaching multidigit multiplication to preservice teachers, though alternate base multiplication appears briefly in some mathematics textbooks for PSTs (Bennett et al., 2012a; Freitag, 2012). There appear to be two distinct purposes for the use of alternate bases in research on PSTs, with one purpose being identifying PST conceptions that would otherwise be hidden in base ten (Thanheiser \& Rhoads, 2009; Zazkis \& Khoury, 1993). The other purpose for alternate bases is to serve as a problematized mathematical context in which PSTs are asked to explore ideas of whole number and operation (McClain, 2003; Thanheiser, 2014; Yackel et al., 2007). In the following sections I discuss each of these purposes in greater detail.

\section{Using Alternate Bases to Reveal Conceptions}

A small body of research examines how PSTs deal with non-decimal decimals, such as $12.34_{\text {five }}$ (Khoury \& Zazkis, 1994; Zazkis, 1999; Zazkis \& Khoury, 1993, 1994). These are fractional numbers written in "decimal" form, though the word decimal could be replaced with the word "pentimal" to refer to the base five domain. "The unfamiliar domain is chosen in order to detect students' difficulties that may not be apparent with the more familiar and overlearned routine-type problems" (Zazkis \& Khoury, 1993, p. 
39). When PSTs were asked to translate $12.34_{\text {five }}$ from base five to base ten, their strategies revealed that many did not see a consistent relationship between columns, where each column was worth five times more than the column to its right.

Misconceptions included treating the pair of columns to the right of the decimal point as (1/5 and 1/50), as (.5 and .05), or as (.5 and .25). These misconceptions, which would have been hidden in base ten, were revealed in base five, indicating that base five is a useful tool for uncovering place value conceptions.

Thanheiser and Rhoads (2009) use the base 20 context of Mayan numbers to explore the meaning of appending extra zeros to a base 20 number. The PSTs had worked previously on Mayan numerals using two place values, but not three place values. The PSTs appeared to be comfortable with the numeral on the left in Figure 2-11 (read as "dot shell") corresponding to one group of 20, but many struggled with the middle numeral "dot shell shell" and the numeral on the right, dot followed by six shells. Rather than interpreting dot shell shell as 20 groups of 20, or 400, some PSTs interpreted dot shell shell as 200 (10 groups of 20) or 100 (1 followed by two zeros). Likewise, a dot with six shells was interpreted as 2,000,000. Similar to the findings of Zazkis and Khoury (1993), the shift in base reveals misconceptions about regrouping that were not apparent in the base ten context. 


\begin{tabular}{|l|c|c|}
\hline & $\bullet$ & 0 \\
Mayan Symbol & Mayan Symbol & 0 \\
Read as $_{\text {"dot shell" }}$ & $\begin{array}{l}\text { Read as } \\
\text { "dot shell shell" }\end{array}$ \\
$10_{\text {twenty }}=20$ & $100_{\text {twenty }}=400$ & $1000000_{\text {twenty }}^{=6}=64000000$ \\
\hline
\end{tabular}

Figure 2-11. In the base twenty Mayan context, a dot followed by one shell refers to one group of 20, a dot followed by two shells refers to one group of $20^{2}$, or 400 , and dot followed by six shells refers to a group of $20^{6}$, or 64000000 .

Both the Mayan activity and the base five decimals suggest potential activities for PSTs to strengthen their understanding of place-value structure. Investigating an alternate base can shift the role of place value from an implicit idea to an explicit construct for PSTs to examine. For example, in her 2015 study, Thanheiser leverages the Mayan task to promote shifts in PSTs conceptions of whole numbers. Thanheiser (2015b) brings together multiple historic numeration systems, leveraging variation theory (M. L. Lo, 2012) to design tasks to help PSTs better understand the structure of number systems.

\section{Using Alternate Bases as a Novel context for Exploration}

The research of McClain (2003) and Yackel et al. (2007) rely on an elementary curriculum modified to a base eight context. Both studies use a candy factory context problematized to base eight, in which eight candies fit in a roll and eight rolls fit in a box.

The reason for modifying the context was that if the mathematics had been trivial for the preservice teachers, the need to create ways to symbolize their transactions would not have emerged naturally. The goal was then to build from the preservice teachers' evolving notational schemes to support shifts in their understandings of place value and multidigit addition and 
Investigating Alternate Numeration Systems

subtraction so that they might develop conceptual understanding instead of mere proficiency with meaningless algorithms. (McClain, 2003, p. 286)

Yackel et al. focused on two instructional sequences, one on number and one on coordinating units (candies, rolls, and boxes). McClain focused more heavily on addition and subtraction in the candy factory context. Both studies found that PSTs were able to experience the early stages of counting and learn about strategies based on number relationships rather than pre-created algorithms. Both studies found that PSTs used learning trajectories similar to those used by children, but that PSTs were not in the same position as children because they had additional prior experience with place value and algorithms.

Considering the two studies as a sequence, with Yackel et al. focusing more on number sense and McClain focusing on addition and subtraction, the next step in the sequence would be my study on the use of an alternate base for teaching multidigit multiplication. The candy factory context may not be appropriate for multiplication, as it may limit the PSTs to a repeated addition model of multiplication rather than the more powerful area model.

\section{Implications for My Research}

Alternate base contexts have proved fruitful for researchers to identify PSTs' conceptions of whole number. This problematized context may also help PSTs to realize that they have something to learn about multidigit arithmetic beyond the use of rote algorithms. An exploration of multidigit multiplication may reveal and support PSTs' developing understanding of the multiplicative structure of place value number systems. What PSTs learn in an alternate base may strengthen their knowledge of base ten. The 
alternate base and base ten become two examples of a more general phenomenon: the place value number system.

In my research I look at the role of alternate bases in current PST textbooks and explore the potential of an instructional sequence leveraging base five as a context for multidigit multiplication. I also explore the idea of problematizing a mathematical context as a design heuristic for supporting PSTs to re-engage with routine content. The problematized mathematical context serves as a source of genuine problems, problems for which PSTs do not have pre-built solution strategies.

\section{Overview}

In the previous sections I have discussed background literature on teacher knowledge, base ten number and operations, and alternate bases. I have also provided key mathematical background information on base ten and alternate bases, as well as multiplication. These components represent necessary background for my study. In the following section I discuss the theory that is used to ground my design research study.

\section{Theoretical Perspectives}

When designing and analyzing a research study, the researcher should be transparent about his or her own theoretical perspectives. In this section I describe the theories that play a central role in my design research study. The theoretical perspectives section is split into three parts. The first part focuses on the emergent perspective (Cobb $\&$ Yackel, 1996), which is the constructivist learning theory that underlies my research. The second part focuses on Harel's instructional principles of duality, necessity, and repeated reasoning (2007), which describe conditions that link teaching activity to successful student learning. The third part describes the design heuristics of Realistic 
Mathematics Education (RME) which guide the design and analysis of the instructional sequence for this study.

\section{Emergent Perspective}

The emergent perspective is a learning theory which attends to both individuals and interactions in a learning environment (Cobb \& Yackel, 1996). The psychological constructivist approach focuses on the mathematical activity of individuals, while the sociocultural perspective focuses on interactions and collective classroom actions. According to Cobb and Yackel, the framework for the emergent perspective "involves the explicit coordination of two distinct theoretical viewpoints on classroom activity" (1996, p. 176). Figure 2-12 illustrates the coordination between the perspectives, where the items in the right-hand column are the individualized component of the social construct on the left-hand side.

\begin{tabular}{|l|l|}
\hline Social Perspective & Psychological Perspective \\
\hline Classroom social norms & $\begin{array}{l}\text { Beliefs about own role, others' roles, and } \\
\text { the general nature of mathematical activity } \\
\text { in school }\end{array}$ \\
\hline Sociomathematical norms & Mathematical beliefs and values \\
\hline Classroom mathematical practices & Mathematical conceptions and activity \\
\hline
\end{tabular}

Figure 2-12. The emergent perspective coordinates the social and psychological perspectives

Classroom social norms refer to the collective expectations held by both the teacher and students, as it relates to day to day classroom experiences. For example, the classroom social norms in a traditional class may involve a question / answer process in which teachers ask focused questions and individual students provide single word answers. Classroom norms for an inquiry-oriented class would involve sharing and justifying solutions and making sense of the solutions of others. Given that a classroom 
includes a collection of individuals, as well as a whole group, it is possible and helpful to view these expectations as belonging to the collective as well as the individuals. Within my study I established norms which supported an inquiry-oriented learning environment. While the classroom social norms described above could apply to many other subject areas, the second construct in Figure 2-12 focuses more narrowly on the subject of mathematics. Sociomathematical norms refer to normative behavior that relates to student mathematical activity, such as determining whether a mathematical solution is valid or efficient, or comparing solution strategies to determine if they are mathematically different. These activities are widely used across the field of mathematics, rather than tied to a single subdomain or developmental level. The third construct outlined by Cobb and Yackel is classroom mathematical practices, which are more narrowly tied to particular mathematical topics. The mathematical practices of a community include the mathematical ideas that are "taken as shared" and no longer require justification each time they are used. For example, in elementary school, when students initially begin to decompose numbers into $10 \mathrm{~s}$ and $1 \mathrm{~s}$, this activity has to be justified. Later in elementary school, this idea shifts to "taken as shared" and no longer requires explanation. For all three constructs within the emergent perspective, the relationship between the social and psychological perspective is reflexive. Rather than seeing changes in one column as causing changes in the other, a research coordinates the two perspectives in order to better understand shifts at both the group and individual levels.

While the establishment of appropriate classroom and sociomathematical norms is important to the implementation of the teaching experiments, my research focuses more 
narrowly on the mathematical practices of the PSTs, in order to develop a local instructional theory for multiplication. In order to support PSTs' development, I leverage the design heuristics of RME (Gravemeijer, 1998), which I discuss next.

\section{RME Instructional Design Heuristics}

In traditional mathematics teaching, the instructor often begins with the result, such as a definition or algorithm, and then provides a series of examples of that result. Proponents of Realistic Mathematics Education (RME) argue that this traditional approach is backwards (Freudenthal, 1991; Gravemeijer \& Terwel, 2000), or an antididactical inversion. RME is an instructional design theory that focuses on beginning with contexts that are realistic or meaningful to students and building up ideas through a process of mathematizing (organizing mathematically), working from examples to more general conceptions. The following three design heuristics support this process of mathematizing.

\section{Guided Reinvention}

Guided reinvention is a design heuristic which focuses on creating an instructional path where students recreate important ideas in mathematics. "The idea was to allow learners to come to regard the knowledge they acquire as their own, personal knowledge, knowledge for which they themselves are responsible." (Gravemeijer \& Terwel, 2000, p. 786) Generally, guided reinvention focuses on building up from student activity in contexts that are experientially real to the students. As students organize or mathematize their ideas, they are able to build up new mathematical realities. This is also referred to as progressive mathematization, which can be broken down into two components: horizontal and vertical mathematization (Gravemeijer \& Cobb, 2006). 
Investigating Alternate Numeration Systems

Horizontal mathematizing involves mathematizing reality. For example, if a student solves a base ten subtraction problem with manipulatives and then records that process with symbols, this activity is horizontal in nature because the student is organizing something that is real to the student. Vertical mathematizing relies on mathematizing prior mathematical activity. Returning to the prior example, if a student then uses this record process to create a general strategy for subtraction without needing to manipulate base ten pieces, this generalizing activity is vertical in nature.

\section{Didactic Phenomenology}

The design heuristic of didactic phenomenology works is conjunction with the design heuristic of guided reinvention. The choice of the mathematical context being investigated within guided reinvention is informed by didactic phenomenology (Bowers, 1995; Gravemeijer \& Terwel, 2000; Whitenack \& Knipping, 2002). Didactic phenomenology is the study (by the instructional designer) of how a particular context/problem/phenomenon can be tapped for sense making and vertical mathematizing by students. The context chosen should be one that begs for organizing from a mathematical perspective (i.e. mathematizing) (Gravemeijer \& Cobb, 2006; Treffers, 1987b). Moreover, the type of mathematizing that students naturally engage in should be the type of mathematizing intended by the instructional designer (Gravemeijer, 1998). The contexts chosen by the instructional designer may be historic in nature, such as contexts that led to key developments in mathematics, or they may be common place contexts that embody a mathematical phenomenon.

As an example, consider the phenomenologically rich context of finding the area of a rectangle. While this could be done by filling the rectangle with units and counting 
one by one, the context invites its users to create more efficient methods to compute the product, such as repeated addition of rows or partitioning the problem into known easier rectangles (such as ten by ten squares). Efficient organizing may lead to implicit or explicit use of the distributive property (which is at the heart of most invented multiplication strategies.) Additional focus on the phenomenon of area reveals its utility in investigations beyond whole numbers, including multiplication of fractions, decimals, and polynomials (See Figure 2-13). These examples of the area model are drawn from my experiencing teaching mathematics ranging from elementary mathematics content courses through calculus.

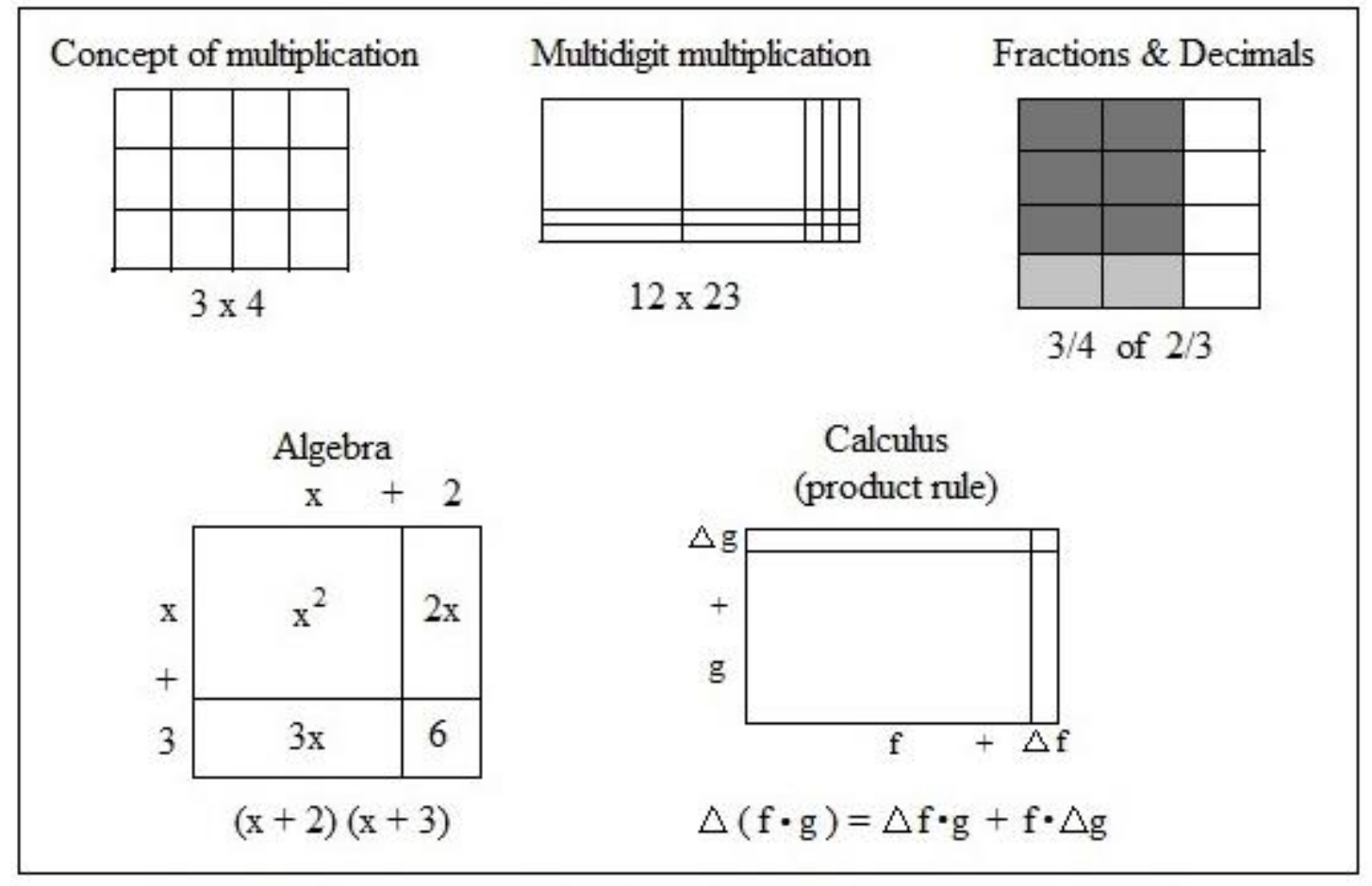

Figure 2-13. Phenomenological analysis of the area model reveals the utility of the model across a variety of number domains. 
Investigating Alternate Numeration Systems

\section{Emergent Models}

As students engage in realistic mathematical contexts, their mathematizing activities can be described in terms of the emergent models they build. These emergent models are initially informal in nature and tied to the local context, but the models can become more formal as students engage in further exploration of similar contexts (Gravemeijer, 1998). The term model is used to refer to students' strategies, notations, ideas, or descriptions. Four levels of emergent models are listed below.

1. Situational level - Students use informal, local strategies situated within contexts that are mathematically real to the students, such as counting base ten pieces to solve an addition problem.

2. Referential level - Students continue to refer to the context without being embedded within it. i.e. Students use symbols to represent activities with whole numbers rather than counting physical blocks.

3. General level-Students focus on strategies rather than references to the particular context. i.e. Students may generate a strategy for any two digit addition problem.

4. Formal level-Students work with conventional notation and procedures. The shift from situational to referential is a horizontal shift, as the focus continues to be on mathematizing the particular context. The shift from referential to general represents a vertical shift as students mathematize their prior (referential) mathematical model. The shift from general to formal is not always a goal of the mathematizing process. For example, learning the traditional U.S. algorithm for long division may not be the intended goal of a RME curriculum for multidigit division. Gravemeijer characterizes emergent 
models with three attribute: their "naturalness" to students, their "vertical power" for mathematizing, and their "breadth of application" across applied situations (1998, p. 290). While the levels of emergent models build up, underlying levels are still accessible to students, who may return from a general to a referential or situated level as needed.

The emergent models are often categorized into two levels instead of four using the models-of / models-for language (Gravemeijer \& Doorman, 1999; Johnson, 2013; Larsen, 2013). This language also captures the idea of a shift from mathematizing a context (creating a model-of the context) to vertical mathematizing as that model becomes a model-for more advanced strategies.

My role as the curriculum designer is to create an instructional sequence with contexts and tasks that lend themselves to horizontal and vertical mathematizing, allowing students to reinvent key ideas in mathematics. The above three RME design heuristics provided guidance in this design process.

\section{Overview \& Research Questions}

Within Chapter 2 I have described the construct of MKT and synthesized literature on gaps in PSTs' knowledge of the mathematics they will need for teaching. I have introduced the problematized context of alternate bases as a potential tool to address gaps in PSTs' knowledge and I have synthesized the current literature on leveraging alternate bases with PSTs. The first of my research questions addresses the role that alternate numeration systems play in current PST curricula.

In this chapter I have also described the design heuristics of RME. The second and third research questions rely on and advance the design theory of RME. I leveraged the RME design heuristics to create an initial local instructional theory for working with 
Investigating Alternate Numeration Systems

multiplication in alternate bases. I also rely on RME theory in reflecting on alternate bases as an example of a wider phenomenon of shifting to non-routine contexts to support guided reinvention of core mathematical ideas.

\section{Research Questions}

(1) How and why are alternate numeration systems being used in PST textbooks?

(2) How can alternate bases be leveraged to support PSTs in reinventing a general strategy for multiplication?

(3) How can problematizing to an alternate base be considered as an example of a wider task design heuristic? 
Investigating Alternate Numeration Systems

\section{Chapter 3 : Methods}

The Methods Chapter is split into four sections, with the first section focusing on the methods for the textbook analysis of the role of alternate bases in preservice teacher mathematics content textbooks. Sections two through four focus on the cyclic design experiment for the creation of a local instructional theory for multidigit multiplication using the context of alternate bases. Section two describes the instructional sequence, including the mathematical tasks and anticipated student activity. Section three describes the participant recruitment and data collection methods. Section four describes the retrospective analysis process which was applied to the video and written data from the teaching experiments.

\section{Section 1: Methods for Textbook Analysis}

In this study, thematic analysis (Braun \& Clarke, 2006) was used to uncover and document themes in the use of alternate numeration systems in preservice teachers (PST) mathematics content textbooks. Thematic analysis was selected because there were no preexisting frameworks for considering roles of alternate numeration systems.

\section{Why Does It Matter That There Are Different Roles for Alternate Systems?}

From the point of view of a teacher educator, the goals of a curricular tool are important when implementing that tool. For example, while one set of textbook directions may focus on converting between base ten and a new base, another set of directions may discourage such conversions. Without knowing the underlying reasoning behind the curriculum, a teacher educator could unintentionally undermine the goals of the curriculum. From the point of view of a researcher, awareness of distinct approaches may help when attempting to synthesize or build upon prior research. If the different 
approaches have different goals and outcomes, this must be taken into account when attempting to draw conclusions from a range of alternate base studies.

\section{Selection of textbooks}

I selected textbooks with the intention of including some of the most commonly used curricula for preservice teachers, as well an including a range of both newer curricula ( $4^{\text {th }}$ edition or less) and older curricula ( $9^{\text {th }}$ or higher edition). Textbook adoption lists were provided by the publishers for each of the seven selected curricula. According to the publishers, five of the curricula had each been adopted by over 150 college and university campuses across the country (Bennett et al., 2012a; Billstein, Libeskind, \& Lott, 2012; Long, DeTemple, \& Millman, 2012; Musser, Peterson, \& Burger, 2011a; Sowder, Sowder, \& Nickerson, 2014). The Beckmann (2014) curriculum has been adopted at over 100 schools and the Bassarear (2012c) curriculum at over 50 schools. While the study does not include every available mathematics curricula for PSTs, the sample does include textbooks which are in common use across the U.S., with all 50 states represented in the sample.

Table 3-1 provides a list of the selected textbooks, alphabetical by author. In the case where there is a textbook and activity book by the same author set, both are included in the analysis ${ }^{5}$. The textbooks used for review are the Instructor Editions provided by the publishers. The three letter code under each author or set of authors in Table 3-1 is used to refer to the curricula in subsequent tables.

\footnotetext{
${ }^{5}$ Two Pearson curricula, Billstein, Libeskind, and Lott (2012) and Long, DeTemple, and Millman (2012), mention activity books created by a different Pearson authoring team (Dolan, Williamson, and Muri). These activity books were not included in the study because of the different authoring team and the minimal inclusion of alternate bases.
} 
Table 3-1. Selected Textbooks

\begin{tabular}{|c|c|c|}
\hline \multicolumn{3}{|c|}{ Mathematics Content Curricula Selected for Inclusion in the Study } \\
\hline Author(s) & Textbook Title(s) & $\begin{array}{l}\text { Edition, Year \& } \\
\text { Publisher }\end{array}$ \\
\hline $\begin{array}{l}\text { Bassarear } \\
\text { (Bas) }\end{array}$ & $\begin{array}{l}\text { Mathematics for Elementary School } \\
\text { Teachers } \\
\text { Explorations: Mathematics for Elementary } \\
\text { School Teachers }\end{array}$ & $\begin{array}{l}5^{\text {th }} \text { Edition, }(2012 \mathrm{c}) \\
\text { Brooks Cole, } \\
\text { Cengage Learning }\end{array}$ \\
\hline $\begin{array}{l}\text { Beckmann } \\
(\mathrm{Bec})\end{array}$ & $\begin{array}{l}\text { Mathematics for Elementary Teachers with } \\
\text { Activity Manual(Beckmann, 2014) }\end{array}$ & $\begin{array}{l}4^{\text {th }} \text { Edition, (2014) } \\
\text { Pearson }\end{array}$ \\
\hline $\begin{array}{l}\text { Bennett, Burton, } \\
\text { \& Nelson } \\
(\mathrm{BBN})\end{array}$ & $\begin{array}{l}\text { Mathematics for Elementary Teachers: A } \\
\text { Conceptual Approach } \\
\text { Mathematics for Elementary Teachers: An } \\
\text { Activity Approach }\end{array}$ & $\begin{array}{l}9^{\text {th }} \text { Edition, (2012) } \\
\text { McGraw Hill }\end{array}$ \\
\hline $\begin{array}{l}\text { Billstein, } \\
\text { Libeskind, \& Lott } \\
\text { (BLL) }\end{array}$ & $\begin{array}{l}\text { A Problem Solving Approach to } \\
\text { Mathematics for Elementary School } \\
\text { Teachers }\end{array}$ & $\begin{array}{l}11^{\text {th }} \text { Edition, }(2012) \\
\text { Pearson }\end{array}$ \\
\hline $\begin{array}{l}\text { Long, DeTemple, } \\
\text { \& Millman } \\
\text { (LDM) }\end{array}$ & $\begin{array}{l}\text { Mathematical Reasoning for Elementary } \\
\text { Teachers }\end{array}$ & $\begin{array}{l}6^{\text {th }} \text { Edition, }(2012), \\
\text { Addison-Wesley } \\
\text { Pearson }\end{array}$ \\
\hline $\begin{array}{l}\text { Musser, Burger, } \\
\text { \& Peterson } \\
(\mathrm{MBP})\end{array}$ & $\begin{array}{l}\text { Mathematics for Elementary Teachers: A } \\
\text { Contemporary Approach } \\
\text { Student Activities Manual with Discussion } \\
\text { Questions for the Classroom }\end{array}$ & $\begin{array}{l}\text { 9th }{ }^{\text {th }} \text { Edition, (2011) } \\
\text { Wiley }\end{array}$ \\
\hline $\begin{array}{l}\text { Sowder, Sowder, } \\
\text { \& Nickerson } \\
(\mathrm{SSN})\end{array}$ & $\begin{array}{l}\text { Reconceptualizing Mathematics for } \\
\text { Elementary School Teachers }\end{array}$ & $\begin{array}{l}2^{\text {nd }} \text { Edition, (2014) } \\
\text { Freeman }\end{array}$ \\
\hline
\end{tabular}

\section{Thematic analysis}

The analysis was done in six phases, corresponding to the six phases described by Braun and Clarke (2006) (see Figure 3-1). These six phases are "(1) familiarize yourself with the data, (2) generate initial codes, (3) search for themes, (4) review themes, (5) define and name themes, and (6) produce the report" (p. 87). These methods are similar to grounded theory research methods (Creswell, 1998) in that there is a focus on open coding, grouping codes, and selecting and defining overarching themes. Unlike grounded 
theory, there is not a focus on repeatedly gathering more data with the intention of reaching saturation. Rather, the goal is to find themes within a given body of data, in this case, a set of PST textbooks.

\begin{tabular}{|c|c|}
\hline Phase & Description of the process \\
\hline $\begin{array}{l}\text { 1. Familiarizing yourself } \\
\text { with your data: }\end{array}$ & $\begin{array}{l}\text { Transcribing data (if necessary), reading and re-reading the data, noting down } \\
\text { initial ideas. }\end{array}$ \\
\hline 2. Generating initial codes: & $\begin{array}{l}\text { Coding interesting features of the data in a systematic fashion across the entire } \\
\text { data set, collating data relevant to each code. }\end{array}$ \\
\hline 3. Searching for themes: & $\begin{array}{l}\text { Collating codes into potential themes, gathering all data relevant to each } \\
\text { potential theme. }\end{array}$ \\
\hline 4. Reviewing themes: & $\begin{array}{l}\text { Checking if the themes work in relation to the coded extracts (Level 1) and the } \\
\text { entire data set (Level 2), generating a thematic 'map' of the analysis. }\end{array}$ \\
\hline $\begin{array}{l}\text { 5. Defining and naming } \\
\text { themes: }\end{array}$ & $\begin{array}{l}\text { Ongoing analysis to refine the specifics of each theme, and the overall story the } \\
\text { analysis tells, generating clear definitions and names for each theme. }\end{array}$ \\
\hline 6. Producing the report: & $\begin{array}{l}\text { The final opportunity for analysis. Selection of vivid, compelling extract } \\
\text { examples, final analysis of selected extracts, relating back of the analysis to the } \\
\text { research question and literature, producing a scholarly report of the analysis. }\end{array}$ \\
\hline
\end{tabular}

Figure 3-1. Phases of thematic analysis, Image from Braun \& Clarke, 2006, p. 87.

When engaged in thematic analysis, it is important for the researcher to be clear about choices they make in the process of data analysis. One major choice is whether the thematic analysis will be theoretical (based on prior frameworks) or inductive (data driven) (Braun \& Clarke, 2006). For my analysis of textbooks I used an inductive approach, relying on the data to create initial themes.

Next I discuss the six phases for thematic analysis as they relate to my textbook analysis.

\section{Phase 1: Get Familiar with the Data}

In the first phase of the data analysis I read the textbook sections that relate to alternate bases for each of the textbooks included in the study. Some of these textbooks I was already familiar with because I have used them in my own teaching. Phase 1 gave me an opportunity to become familiar with the full set of textbooks under analysis. Part of Phase 1 involved jotting down initial notes about ideas within the data. As the 
researcher, my thoughts, questions, and wonderings played a central role in the analysis of data. I used an analysis log to record these thoughts.

There are a variety of ways to read a mathematics textbook. One option is to read it as a student learning mathematics. Another option is to read it as a teacher, preparing to teach a topic from the textbook. Yet another option is to approach the textbook with a particular research question or set of questions in mind. This third option was the approach I used, allowing my research questions to guide my reading. My research questions are: How are alternate numeration systems leveraged in PST content curricula? and What are the rationales provided by the curricula for why PSTs should study alternate systems? As a researcher, the following sub-questions also served to guide my reading and help me to examine the textbooks for a variety of purposes.

- What are the intended purposes of the segment? For example, does the segment focus on number or operation, historical background, or children's thinking?

- What links, if any, are being made between base ten and the alternate base? Or between multiple alternate bases?

- What types of bases are being used? (Historic, etc.)

As a part of Phase 1 I scanned the relevant pages of the textbooks to create digital copies which were used for coding in Phase 2. I used a qualitative data analysis software package $\left(\right.$ HyperResearch ${ }^{\mathrm{TM}}$ ) as a tool for the coding process.

\section{Phase 2: Generate Initial Codes}

In Phase 2, I split the textbook data into segments corresponding to separate examples, explanations, and sets of related exercises. There were generally 3 to 8 segments per page, though some exercise sections had more segments. During the initial 
creation of codes, the goal was to describe attributes of the data. This process is similar to open coding, defined by Strauss and Corbin as the "analytic process through which concepts are identified and their properties and dimensions are discovered in data" (1998, p. 101). Individual segments could receive multiple codes describing different aspects of the segment. As new codes were created I continually looked back and compared to prior codes.

An important aspect of coding is to be able to examine many instances of a particular code. Qualitative data analysis software was used to organize examples of each code.

\section{Phase 3: Search for Themes}

After the data was coded in Phase 2, I searched for initial themes across the codes. This process involved grouping codes from Phase 2 into logical categories. Braun and Clarke describe this process as "[e]ssentially, you are starting to analyse your codes and consider how different codes may combine to form an overarching theme" (2006, p. 89). A concept map of the codes can help in the process of creating themes. Phase 3 was used to create potential themes which were reviewed in the next phase.

\section{Phase 4: Review Themes}

Phase 4 was split into two stages. The first stage involved rereading the data segments that correspond to each theme to determine whether they formed a coherent category of ideas. In this stage I considered whether the themes were clearly distinguishable from one another. During this stage, some themes were shifted, and some parts of the data were recoded to fit within another theme. The second stage of Phase 2 involved looking at the full data set again, determining whether the themes seem to fit the 
data set and checking for data that may have been missed in Phase 2 of the coding process.

Braum and Clarke warn that the process of reviewing and revising themes can become a circular trap for researchers (2006). They encourage researchers to stop once the themes seem to fit well and further retooling of the themes only add minor improvements.

\section{Phase 5: Define and Name Themes}

Once the themes were established in Phase 4, it was time to name and describe the themes. The goal at this phase was to identify and describe the "essence" of each theme, writing a clear and detailed analysis for each theme (Braun \& Clarke, 2006).

"You do this by going back to collated data extracts for each theme, and organizing them into a coherent and internally consistent account,with accompanying narrative. It is vital that you do not just paraphrase the content of the data extracts presented, but identify what is of interest about them and why." (Braun \& Clarke, 2006, p. 92).

While the themes had working titles during Phases 3 and 4 , I followed the advice of Braun and Clarke to give additional thought to the names of themes in Phase 5 to provide brief, clear titles which provide the reader an immediate sense of each theme and subtheme.

\section{Phase 6: Produce the report}

Braun and Clarke describe a report of thematic analysis as "tell[ing] the complicated story of your data in a way which convinces the reader of the merit and validity of your analysis” (2006, p. 93). This story must be grounded in data and provide evidence of the themes, but must go beyond listing examples of the themes. 
Investigating Alternate Numeration Systems

Examples must be embedded within a narrative that makes an argument relating the data to the research question (Braun \& Clarke, 2006).

My goal was to describe a framework for the roles of alternate numeration systems in PST mathematics curriculum, revealing trends in ways of using alternate numeration systems, as well as trends in rationales provided by the curricula.

Next I provide a few examples of the first steps in the thematic analysis by looking at segments from several textbooks together with potential codes for those segments.

\section{Examples from Textbooks:}

In the first example provided in Figure 3-2a, PSTs are shown how to convert from base five to base ten. In another textbook, this is referred to as "[finding] the value of each base-five numeral" (Freitag, 2012, p. 141), which seems to imply that the base-five numeral does not currently indicate the value of the number in question (see Figure 3-2b). This type of problem focuses explicit attention on using the structure of base five to convert the number to a more common notation system, rather than working with the base five number in the context of base five. This stands in contrast to the type of problem in Figure 3-2c, which is solved without leaving the context of base five. In this case, the structure of base five is used to find the number that is one less than the given number. 


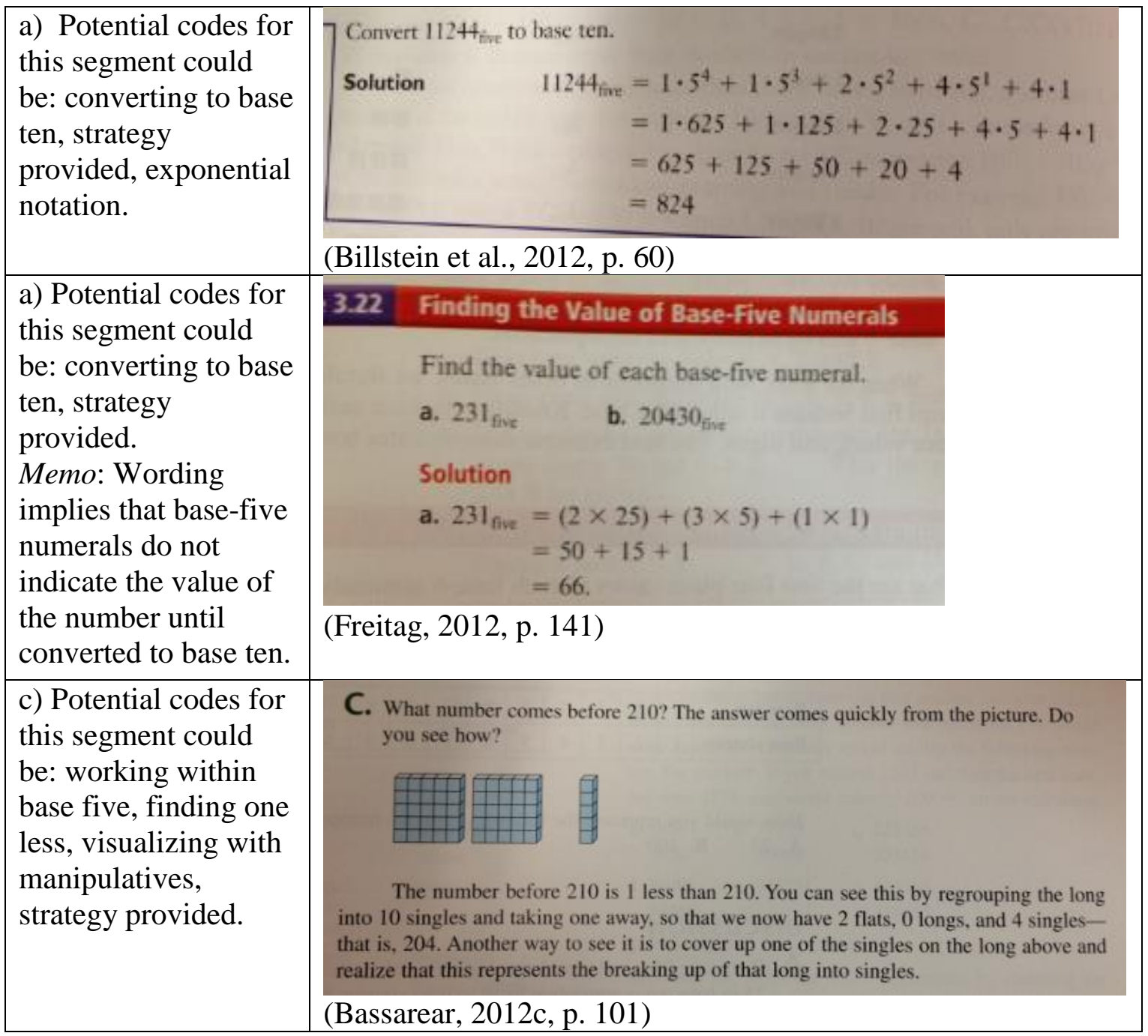

Figure 3-2. Examples of the use of alternate bases in textbooks

\section{The Purpose of the Framework}

The framework generated through the grounded theory process is intended to serve several purposes. First, it opens a discussion about differences in PST curricula which may reveal differences both in types of knowledge goals embedded in curricula and strategies for achieving those goals. Second, the analysis of the role of alternate bases in existing curricula informs the development of potential instructional sequences, such as my instructional sequence for base five multiplication. Third, the framework 
created in Phase 5 may generalize to other content areas which are purposefully problematized for students, such as studying non-Euclidean geometry to better understanding Euclidean geometry.

\section{Overview of Design Experiment}

The next three sections of the methods chapter discuss the format of the teaching experiment for the creation of an initial local instructional theory for multiplication. The

study consisted of two teaching experiment cycles. Each teaching experiment involved working with a pair of students as they engage with the instructional sequence. I focused on the collective mathematical activity of the students, using the lens of the emergent perspective (Cobb \& Yackel, 1996) to focus on both individual and group mathematical activity in order to trace the development of key mathematical models.

Each cycle of the teaching experiment followed the "design, enactment, analysis, and redesign" model outlined by the Design-Based Research Collective (2003, p. 5). The first cycle was conducted at the beginning of Summer 2014 and the second cycle at the beginning of Fall 2014.

\section{Section 2: Task Design for Teaching Experiment:}

\section{A conjectured local instructional theory and task sequence}

Within this section I briefly describe the creation of the task sequence used in the teaching experiment. Appendix B contains an extensive explanation of the task sequence, including anticipated student mathematical activity for each of the tasks in the task sequence, along with additional pre and post survey questions.

The creation of an initial local instructional theory (LIT) for reinventing a general strategy for multidigit multiplication began with a thought experiment about how PSTs 
could move from simpler to more complex multiplication problems and shift from situated to more general solution strategies. I leveraged the design heuristic of problematizing the mathematical context (Chapter 6) to shift from base ten to an alternate number system in order to place PSTs in a position where they do not have formal mathematics for performing multiplication. I initially conjectured that by sequencing multiplication tasks from single digit to two or more digits and including a specific focus on multiplication by the base ( $\left.10_{\text {five }}\right)$, PSTs would reinvent strategies for multiplication which leveraged splitting problems into partial products based along place value lines. I anticipated that a partial products strategy would initially emerge for specific problems and that this situated model of partial products could be generalized into an algorithm for multiplying any pair of base five numbers.

In pilot studies, I found that PSTs tended to rely on symbolic calculations when engaging with multidigit multiplication, performing operations on separate digits within a multiplication task without connecting to an appropriate definition of multiplication. When an area context was introduced within the pilot studies (either by the instructor or a PST), PSTs were able to leverage this meaning of multiplication to advance their multiplication strategies. This led me to revise the local instructional theory to support PSTs' emerging models for the meaning of multiplication alongside their emerging strategies for performing multiplication.

The first step of the LIT involves PSTs establishing multiplication as repeated addition, foregrounding this primary meaning of multiplication as a starting point. The second step of the LIT is developing a strategy for multiplication by the base ( $\left(10_{\text {five }}\right)$, a key step in creating multiplication strategies that leverage the structure of the place value 
number system. The third step of the LIT involves PSTs expanding their model for the meaning of multiplication to include area and array imagery. This third step supports the emergence of a more sophisticated model for the meaning of multiplication as larger problems are introduced and repeated addition becomes untenable. The fourth step of the LIT begins with subdividing the area representation into partial products and shifts to breaking numbers efficiently along place value lines. Within this fourth step, PSTs' model of partial products becomes a model for multiplying any pair of numbers, resulting in a general multiplication strategy.

The multiplication task sequence for the teaching experiment was created using the initial LIT described above. The sequence of tasks (see Figure 3-3) is intended to promote student mathematical activity in a way that leads to the reinvention of a general strategy for multidigit multiplication. 


\section{Task 1: Repeated Addition \\ a) Find the product $3_{\text {five }} \times 23_{\text {five }}$ and explain your solution strategy. \\ b) Create a definition of multiplication that would be appropriate to use with a $2^{\text {nd }}$ grader.}

\section{Task 2: Times Base Rule}

Find the product $10_{\text {five }}$ x $23_{\text {five }}$ and explain your reasoning. Connect your explanation to a visual model.

Generalizations:

- Make a conjecture about multiplying by $10_{\text {five. }}$ Justify your conjecture.

- Comparison across bases: 10 × $23=230$ and $10_{\text {five }} \times 23_{\text {five }}=230_{\text {five }}$. Is this a coincidence or something more?

\section{Task 3: Measuring a rectangle in base five}

(Each small group receives an unlabeled rectangle, size $42_{\text {five }} \mathrm{X}$ $31_{\text {five }}$ )

Your goal is to examine the size of the rectangle in base five. Determine the length, width, and area of the rectangle and discuss how the three quantities (length, width, and area) are related. Record two or more strategies for calculating the area

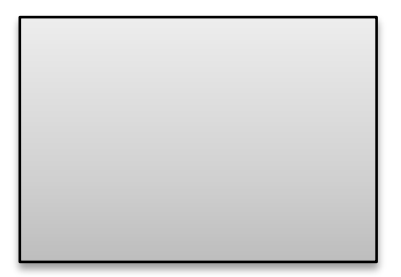
and explain why those strategies make sense. Be sure to connect your visual models with written solutions.

Follow-up question: (Connecting repeated addition and area)

- In the first two base five problems, we used the repeated addition model for multiplication. How is your approach for Task 3 similar to the repeated addition model and how is it different than the repeated addition model?

\section{Task 4: Creating a general strategy}

Alice and Bob, who live in the land of Base Five, have a large box of rectangles. They need a strategy to find the area of any rectangle in base five. Your task is to create and explain an efficient strategy that will work to multiply any two base five numbers. Record your strategy on the provided paper.

Follow-up questions:

- Compare strategies with classmates

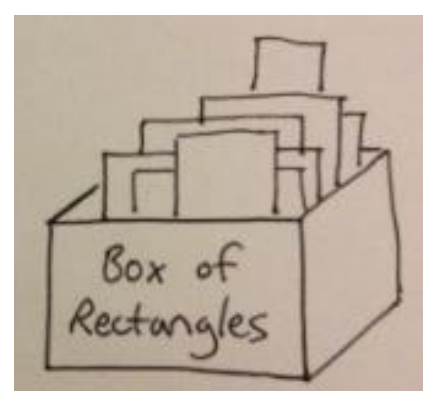

- Explain why the strategy makes sense

- Explain what is efficient about the strategy

- What is the role of the distributive property in each strategy?

- Describe a strategy for multiplying two base five numbers without having to draw a rectangle.

Figure 3-3. Multiplication Task Sequence 
Investigating Alternate Numeration Systems

\section{Section 3: Participant Recruitment and Data Collection for Design Experiment}

\section{Participants}

The participants in this study were preservice elementary teachers in the first term of a three term mathematics content sequence at a large urban university in the Pacific Northwest. The first course in the three-course sequence focuses on whole number and operation. The PSTs in the first cycle of the teaching experiment, Eli and Wendy, volunteered to participate in the task sequence as part of a four-week summer version of the course. The PSTs in the second cycle of the teaching experiment, Nora and Sarah, volunteered to participate in the task sequence in the two weeks prior to the start of the fall term as a head-start for the course on whole number and operation. All names used are pseudonyms. Brief descriptions of the recent math coursework for all participants are shown in Table 3-2.

Table 3-2. Study Participants

\begin{tabular}{|l|l|}
\hline Participants' Recent Mathematics Coursework \\
\hline Cycle 1 Participants & Cycle 2 Participants \\
Eli - Long break since prior math course & Nora - Freshman, had recently taken \\
(calculus) & algebra course \\
Wendy - Repeating the elementary & Sarah - Long break since previous math \\
content course after a prior low grade & course (prior math courses in the military) \\
\hline
\end{tabular}

All class sessions of the teaching experiment were video recorded and student work was collected and scanned. Approximately half of the teaching sessions were attended by a second researcher and the events of the sessions were discussed in debriefing meetings. After each teaching experiment, I wrote a reflection of what had transpired in the class sessions. Original video, transcriptions from the video, and my own notes all served as data sources, along with copies of student written work. 
Investigating Alternate Numeration Systems

For both cycles, my own written reflections about the daily teaching sessions were also considered as a part of the data, as well as research notes made during the analysis of video and written work. The following section details my plans for the retrospective analysis of both cycles of the teaching experiment.

\section{Section 4: Retrospective Analysis of Teaching Experiments}

After the teaching episodes, I engaged in retrospective analysis of the student mathematical activity, as captured on video and in written work. Steffe and Thompson caution that this aspect of the research is "more labor-intensive than the activity of teaching" (2000, p. 296). Although the teacher-researcher is a witness to the teaching experiment, it is the retrospective analysis that provides the opportunity for a thorough historical analysis of what occurred. Having an idea of what mathematical models emerged, I was able to attend to what student mathematical activity led to those models, noting important steps or missed opportunities along the path (Steffe \& Thompson, 2000).

The retrospective analysis of the teaching experiment was an iterative process, which I separated into four major phases, with the first two phases repeated after each cycle of the teaching experiment and the latter two phases analyzing cross the cycles (Figure 3-4). The analysis is based on the cyclic process described by Lesh and Lehrer (2000) and Cobb and Whitenack (1996), and modeled after a cyclic design experiment conducted by Larsen (2004). 


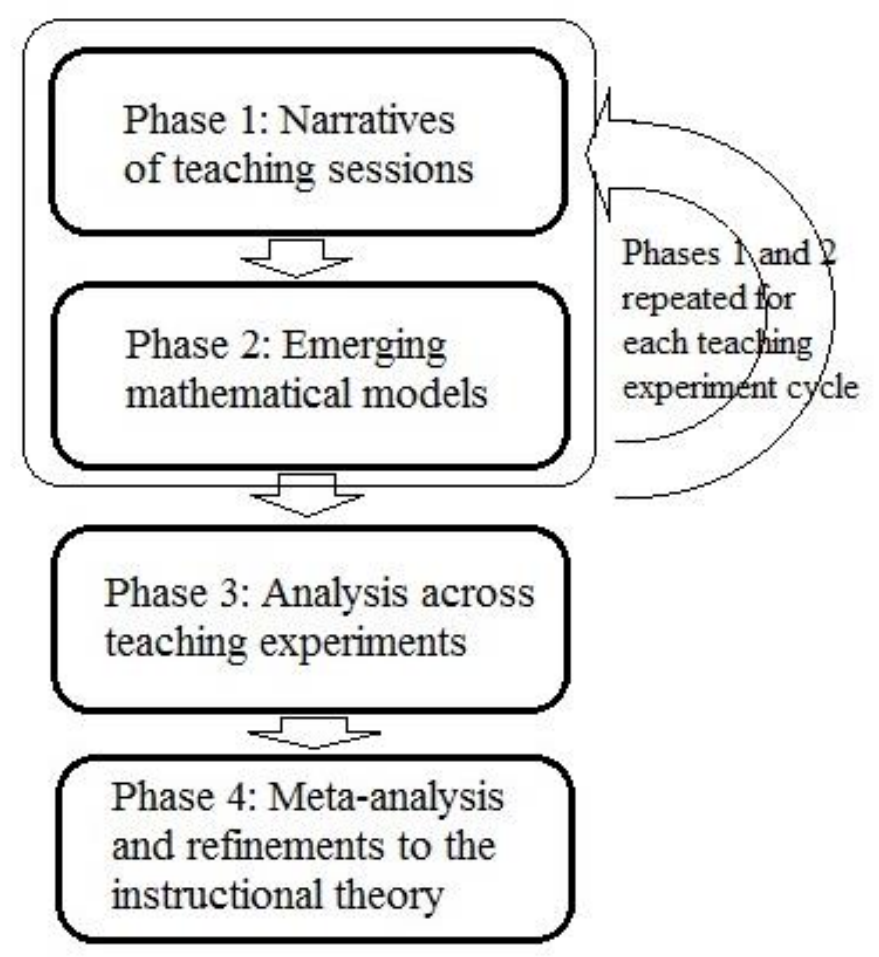

Figure 3-4: Phases of retrospective analysis, with the first two phases repeated for each cycle and the latter two phases conducted across multiple cycles.

\section{Phase 1: Creating Narratives of the Teaching Sessions}

In Phase 1, I worked chronologically, creating a content log of the videos for each teaching session. In order to manage the data, I chunked it into coherent episodes. For this study, an episode refers to a collection of dialogue and student work that corresponds to one solution attempt or one line of reasoning. Episodes often centered around moments of struggle, new strategies and new definitions, discussions of procedures, comparisons across bases, generalizations, and justifications.

An example of a brief episode is shown in Figure 3-5, where Eli and Wendy are responding to a prompt to create a definition for multiplication. This PST generated definition captures their understanding of multiplication as repeated addition, making sense of the first number as the multiplier and the second as the size of the multiplicand. 
Each session contained many episodes, resulting in far more data than could be included in this report. I selected episodes for this report in order to (a) highlight the overall trajectory of PSTs' mathematical activity and (b) focus attention on the co-development and interplay (co-emergence) of PSTs' models for the meaning of multiplication and their models for strategies for performing multiplication.

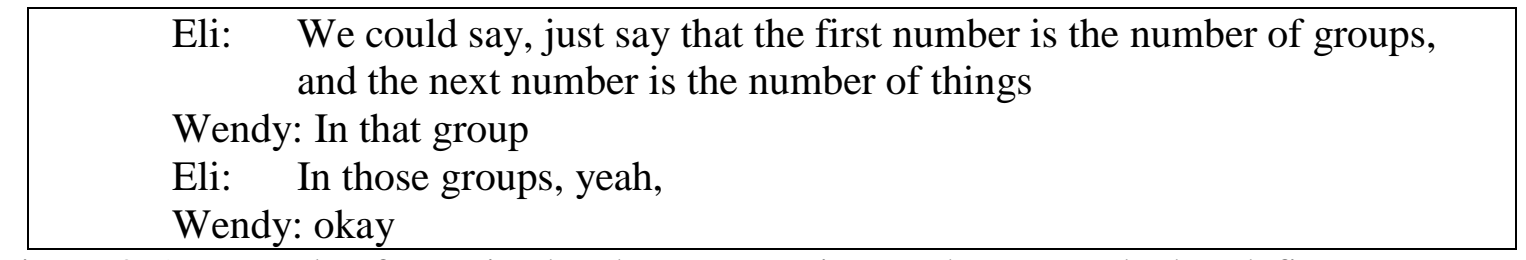

Figure 3-5. Example of an episode where preservice teachers are asked to define multiplication in language appropriate for children.

I created a narrative of each session. This narrative included descriptions of episodes and transcriptions of selected portions of the session, as well as images of student work. The narrative was annotated with conjectures for explanations of student mathematical activity. The goal of phase 1 was to become familiar with the data and to begin to organize the data.

\section{Phase 2: Emerging Mathematical Models}

For each cycle of the teaching experiment, I built detailed reports of the reinvention of the repeated addition and area models of multiplication and the partial products strategy for multiplication. The reports included descriptions of student mathematical activity supported by transcript, photographic evidence, and student written work. I used the design heuristic of emergent models as a lens to describe shifts in the student mathematical activity.

In Phase 2, I included an explicit focus on aspects of the tasks, including student dialogue and questions, which impacted the development and shifted the use of the 
Investigating Alternate Numeration Systems

models. Existing research and domain specific frameworks, such as Graeber, Tirosh, and Glover's (1989) work with PSTs' primitive models for operations and Baturo and Nason's (1996) research on PSTs' understanding of area measurement were used to provide rationales and insights for students' mathematical activities.

While Phases 1 and 2 were done separately for each of the 2 cycles of the teaching experiment, Phase 3 focused on analyzing across both cycles.

\section{Phase 3: Analyze Across Teaching Experiments}

The goal of Phase 3 was to analyze student mathematical activity for particular tasks across the cycles of the teaching experiment. For example, I examined how the PSTs worked with Task $2\left(10_{\text {five }} \times 23_{\text {five }}\right)$ to explore (1) how students made sense of multiplying by the base $\left(10_{\text {five }}\right)$, (2) what sorts of generalizations they were able to make, and (3) what appeared to prompt those generalizations. For example, one local generalization was that multiplying by $100_{\text {five }}$ causes the digits of a number to shift two places to the left, while a more global generalization is that multiplying by the base in any alternate base system causes the digits of a number to shift to the left one place. The reports generated in Phase 2 played a central role in the analysis for Phase 3.

\section{Phase 4: Meta-analysis and Refinements of the Instructional Sequence}

Similar to the final phase of analysis described by Cobb and Whitenack (1996), in this phase I analyzed the prior analyses with the intention of creating a chronology of the emergence of models across the two cycles of the teaching experiment. This chronology drew from both cycles and served as the basis to consider modifications to the instructional sequence and anticipated student mathematical activity. 
During this phase, I searched for common themes or strands to create a story of how a general sense-making multidigit algorithm emerged from student activity. I examined aspects of the instructional sequence (including tasks, mathematical properties, teacher presses, and student dialogue) that played a role in the creation of a sense-making algorithm. It was during this phase of meta-analysis that I stepped past leveraging the tools of RME and considered ways to advance RME theory. I considered the complex situations of how to make sense of student mathematical activity as students developed two distinct, but related models for multiplication. How could I capture the interaction of the two emerging models? This co-development, which I refer to as co-emergence of models, is a focus of the results section of Chapter 5 and serves as a lens or tool to organize students' mathematical activity throughout the task sequence.

\section{Overview}

In the three previous chapters I have (1) discussed why this study is important and how it adds to the existing research literature, (2) provided background literature and theoretical perspectives in order to situate the study, and (3) described my methods for conducting both a textbook analysis and a design research study in the context of alternate numeration systems. In the next three chapters, I present the findings from my study in the form of three self-contained research articles. Chapter 4 is a research report on the role of alternate numeration systems in PST mathematics textbooks. This report highlights two dominant ways in which alternate numeration systems are leveraged; comparing across numeration systems to better understand the mathematical structure of base ten and immersing within a number system to better understand the process of learning a number system. Chapter 5 is a research paper focused on a design heuristic for 
Investigating Alternate Numeration Systems

working with multiple co-developing mathematical models. I present this design heuristic within the context of a teaching experiment leveraging a base five multiplication sequence. I provide an analysis of the co-emergence of two mathematical models; a model for the meaning of multiplication and a model for performing multiplication. Chapter 6 is a theoretical report focusing on a design heuristic for problematizing mathematical contexts. I situate this design heuristic within the design theory of RME and I provide examples from alternate bases and non-Euclidean geometry. 
Investigating Alternate Numeration Systems

\title{
Chapter 4 : The Role of Alternate Numeration Systems in Curricula
}

The Role of Alternate Numeration Systems

in Mathematics Textbooks for Preservice Teachers

\begin{abstract}
Alternate numeration systems are a common feature in mathematics content courses for preservice teachers, but there is a gap in research on how they are leveraged within these courses. Leveraging textbooks as a proxy for enacted curriculum, I analyzed the roles of alternate numeration systems in mathematics content textbooks using thematic analysis to uncover distinct purposes for alternate systems. I identified two primary roles for alternate systems: (1) comparison between base ten and alternate systems, and (2) immersion within an alternate system. I link these roles with textbook rationales for better understanding base ten and better understanding the learning experiences of children. I connect the multiple roles for alternate numeration systems to components of mathematical knowledge for teaching (Ball, Thames, \& Phelps, 2008).
\end{abstract}

In order to teach elementary mathematics well, preservice teachers (PSTs) need to develop strong mathematical knowledge for teaching (MKT) (Ball et al., 2008; H. C. Hill et al., 2008). High levels of MKT are connected to high quality mathematics teaching (H. Hill et al., 2012) and improved student performance (Ball et al., 2005). Researchers have shown that PSTs do not have sufficient MKT in the content area of whole number and operations (Ball, 1990b; Ball et al., 2005; J.-J. Lo et al., 2008; Ma, 1999; Ross, 2001; Thanheiser, 2009a), a content area at the center of elementary mathematics (National Council of Teachers of Mathematics, 2000; National Governors Association Center for Best Practices \& Council of Chief State School Officers, 2010).

Elementary mathematics content courses provide a context for PSTs to develop MKT. However, researchers have found that often PSTs believe they already know enough mathematics to teach (Philipp et al., 2007; Thanheiser et al., 2013), and this can 
make it difficult to engage PSTs in developing a deeper understanding of routine topics. One strategy for helping PSTs to re-engage with familiar mathematical content is to problematize the mathematical context (Chapter 6). I use the phrase problematizing the mathematical context to capture the idea of shifting from a routine mathematical context, such as base ten, to a related, but non-routine context, such as base five or other alternate numeration systems. Problematized mathematical contexts provide opportunities for PSTs to engage in genuine problem solving, engaging with mathematical tasks for which they do not have ready-made solution strategies. (Advantages of problematizing are discussed further in Chapter 6.)

Although there has not been a wide-scale study on the use of alternate numeration systems in PST mathematics content courses across the United States, the common appearance of alternate systems in PST mathematics textbooks (e.g. Bennett, Burton, \& Nelson, 2012a; Billstein, Libeskind, \& Lott, 2012) indicates that they are likely in wide use in teacher education. This raises a question about how alternate systems are being leveraged to support PSTs' mathematical learning. What is the role of alternate numeration systems in PST courses?

One approach to answering this question is an examination of the written curricula for elementary mathematics content courses. The importance of written curricula is captured by Ball and Cohen (1996), who argue that "instructional materials are concrete and daily. They are the stuff of lessons and units, of what teachers and students do. That centrality affords curricular materials a uniquely intimate connection to teaching" (p. 6). Written curricula can serve as a starting point for the content that is presented in the classroom. It sequences content, provides activities and exercises, and 
serves as a mathematical authority. Although what is written in the curriculum is not a perfect match for what happens in the classroom (Stein, Remillard, \& Smith, 2007), the widespread adoption of written curricula provides clues into what is happening in preservice mathematics classrooms across the country.

Textbooks also serve as a key artifact for exploring differences in how specific content is presented. Consider the textbook excerpts in Figure 4-1, capturing opposing views on how to treat base ten while working with an alternate base. On the left, a textbook explicitly compares base ten to an alternate base, using the structure and notation of base ten to explain how base five is structured and recorded. On the right, a textbook advocates avoiding thinking of base ten. In this activity, PSTs are encouraged to work within an alternate number system without translating or comparing to base ten. These divergent uses of alternate bases imply that there are multiple ways of leveraging alternate systems and that those different ways may be linked with different underlying goals.

Alternate bases: Connecting to base ten.
"What number follows $44_{\text {five? }}$ ? There are no
more two-digit numerals in the system after
$44_{\text {five. In base-ten, the same situation occurs at }}$
99. We use 100 to represent ten $10 \mathrm{~s}$, or one
100 . In the base-five system, we need a symbol
to represent five $5 \mathrm{~s}$. To continue the analogy
with base ten, we use $100_{\text {five }}$ to represent one
group of five $5 \mathrm{~s}$ or $5^{2}$, zero groups of five, and
zero units."
(Billstein et al., 2012, p. 60 )

Figure 4-1. Different directions - One text advocates not thinking about base ten while the other draws explicit comparisons between base ten and the alternate base.

In this paper I argue that there are two dominant roles for alternate numeration systems as they appear in PST mathematics curricula. First, alternate systems are used to

Alternate bases: Avoiding base ten
"You will learn far more from this
exploration if you resist the temptation
to think of base ten. Try to get into the
role of an Alphabitian: you have never
heard of base ten. Therefore the
symbols $1,2,3,4$, etc., should not be
used."
(Bassarear, 2012b, p. 104)

\section{Alternate bases: Avoiding base ten} exploration if you resist the temptation to think of base ten. Try to get into the role of an Alphabitian: you have never symbols $1,2,3,4$, etc., should not be used."

(Bassarear, 2012b, p. 104) 
help PSTs develop better understanding of base ten by providing alternate systems to compare against. In addition to this comparative approach, textbooks also offer activities that involve immersing with alternate numeration systems to better understand the process of learning about a number system.

Next I provide a brief introduction to alternate numeration systems. I discuss the mathematics of alternate systems as well as research on leveraging alternate systems for work with PSTs. I then elaborate on the components of MKT, which will be connected to various learning opportunities supported by alternate numeration systems.

\section{Background on Alternate Numeration Systems}

An alternate numeration system is a number system that differs from the base ten (Hindu-Arabic) number system in a structural manner (beyond a superficial relabeling of symbols). Mathematics content textbooks for PSTs commonly include historic systems such as Egyptian, Roman, Mayan, and Babylonian, as well as other historic systems. Egyptian and Roman systems are based on an additive structure rather than place value. That is, the $\mathrm{X}$ in Roman numerals refers to 10 , while $\mathrm{XXX}$ is $10+10+10$ (or 30). The location of an $\mathrm{X}$ does not change its value ${ }^{6}$. On the other hand, base ten is a place value system where the position of a digit does impact its value. For example, the 3 in 234 represents thirty, while the 3 in 324 represents three hundred.

While Egyptian and Roman numeration systems differ from base ten because of their additive structure, Mayan and Babylonian differ from base ten because of their base.

\footnotetext{
${ }^{6}$ In the Roman system, higher value symbols are usually placed to the left of lower value symbols, indicating the symbols should be summed. E.g. XI $=(10+1)=11$. Swapping the order of the symbols is an indicator to subtract the smaller value from the larger. E.g. IX $=10-1=9$. Despite this subtractive property, the symbol X cannot take on any value other than ten. For more information, see Musser, Peterson, and Burger (2011a).
} 
The base of a number system is the quantity needed for regrouping. In base ten, each time ten of one unit is collected, it can be regrouped to one of the next larger unit. For example, 10 tens is one hundred and 10 hundreds is one thousand. The Maya system ${ }^{7}$ is based on regrouping at 20 and the Babylonian system is based on regrouping at 60 . A legacy of the Babylonian system is the division of an hour into 60 minutes and a minute into 60 seconds.

In addition to historic numeration systems, PST textbooks often contain modern alternate base systems, such as base five, hexadecimal, and binary. I refer to these systems as modern alternate base systems due to the use of notation similar to base ten, including modern digits $(0,1,2,3, \ldots)$. When additional digits are needed, they are typically borrowed from the alphabet. For example, hexadecimal uses the sixteen symbols 0 through 9 and A through F. An example of base five is provided in Figure 4-2. In the base five number system each place value is associated with a power of five. There is a ones place $\left(5^{0}\right)$, a fives place $\left(5^{1}\right)$, a twenty-fives place $\left(5^{2}\right)$, and so on for each power of five. Only the symbols $0,1,2,3$, and 4 are needed as regrouping occurs once you reach five of one type of unit.

\begin{tabular}{|l|l|}
\hline \multicolumn{1}{|l|}{$|l|$} \\
$\begin{array}{l}\text { a) The quantity corresponding to } 69 \text { in base } \\
\text { ten can be recorded as } 2 \text { groups of twenty- } \\
\text { five, } 3 \text { groups of five, and } 4 \text { ones. This is } \\
\text { written as } 234_{\text {five }} \text { where the subscript five } \\
\text { indicates the base. }\end{array}$ & $\begin{array}{l}\text { b) The imagery of base pieces can be } \\
\text { used without filling in the shapes. In } \\
\text { this way, the image could refer to }\end{array}$ \\
\hline
\end{tabular}

Figure 4-2. An example of the base five number system and a general number system.

\footnotetext{
${ }^{7}$ The Maya system is sometimes presented as purely base twenty and other times matches the historic convention where the third place value is regrouped at 18 rather than 20, following the Maya calendar of 18 months with 20 days each. For more information, see Long et al. (2012).
} 
Once you are familiar with a few alternate base systems, alternate bases can be approached generally. Figure $4-2 b$ provides base imagery which is general enough to use for many bases.

\section{Research on Alternate Bases and Preservice Teachers}

Alternate numeration systems have been used by researchers to reveal PST conceptions that would be hidden in base ten (Thanheiser, 2015a; Thanheiser $\&$ Rhoads, 2009; Zazkis \& Khoury, 1993, 1994). Zazkis and Khoury (1993) note that "[t]he unfamiliar domain is chosen in order to detect students' difficulties that may not be apparent with the more familiar and overlearned routine-type problems" (p. 39). Researchers have also leveraged alternate numeration systems to serve as a context for tasks which allow PSTs to explore and reinvent ideas in the domain of whole numbers and operations (Fasteen, Melhuish, \& Thanheiser, 2015; McClain, 2003; Thanheiser, 2014; Yackel et al., 2007). For example, the research of McClain (2003) and Yackel et al. (2007) relies on an elementary curriculum modified to a base eight context for use with PSTs. A goal of these studies was to help PSTs to "develop conceptual understanding instead of mere proficiency with meaningless algorithms" (McClain, 2003, p. 286). McClain notes that the alternate base context was also used for the pedagogical purpose of having PSTs reflect on their own learning and their future roles as teachers. She found that the PSTs struggled to reflect on their future roles as teachers while also working to make sense of the mathematics. However, the PSTs were able to step back and reflect after the learning process and consider how their learning experiences mirrored the learning experiences of children. This led to a shift in the PSTs view of learning elementary mathematics. McClain notes that the PSTs' "goals for their 
classrooms were shifting from a focus on correct procedures to an emphasis on students' understanding in the context of their mathematical activity" (pp. 303-304).

Researchers show that alternate numeration systems are a promising context for allowing PSTs to revisit routine ideas of whole number and operation (Fasteen et al., 2015; McClain, 2003; Thanheiser, 2014; Yackel et al., 2007). They allow PSTs to focus more deeply on the underlying structure of place value and its role in making sense of multidigit operations. In order to become prepared to teach elementary students about whole number and operation, PSTs must develop a more explicit awareness of the structure of base ten than those who use mathematics in other professions. In the next section I describe different types of knowledge that are called upon by elementary mathematics teachers.

\section{Theoretical perspective}

\section{Mathematical knowledge for teaching}

Teaching mathematics is a complex task. In order to teach well, teachers must know more than just the set of concepts and procedures they teach. They must also know how to unpack the mathematics, how to work with student conceptions, and how to select appropriate representations for specific content (Ball et al., 2008; H. C. Hill et al., 2008). Ball et al. (2008) describe a framework for different domains of mathematical knowledge for teaching (MKT), first splitting MKT into the two major components of subject matter knowledge and pedagogical content knowledge, and then splitting each of those components into three subsections (see Figure 4-3). Each of these subsections is described below. For this study, the goal of splitting MKT into components is not to 
imply that PSTs should learn each component separately. Rather, viewing MKT as a collection of components serves as a useful lens to consider how PSTs may begin to develop MKT and to consider how investigations of alternate bases can support these components.

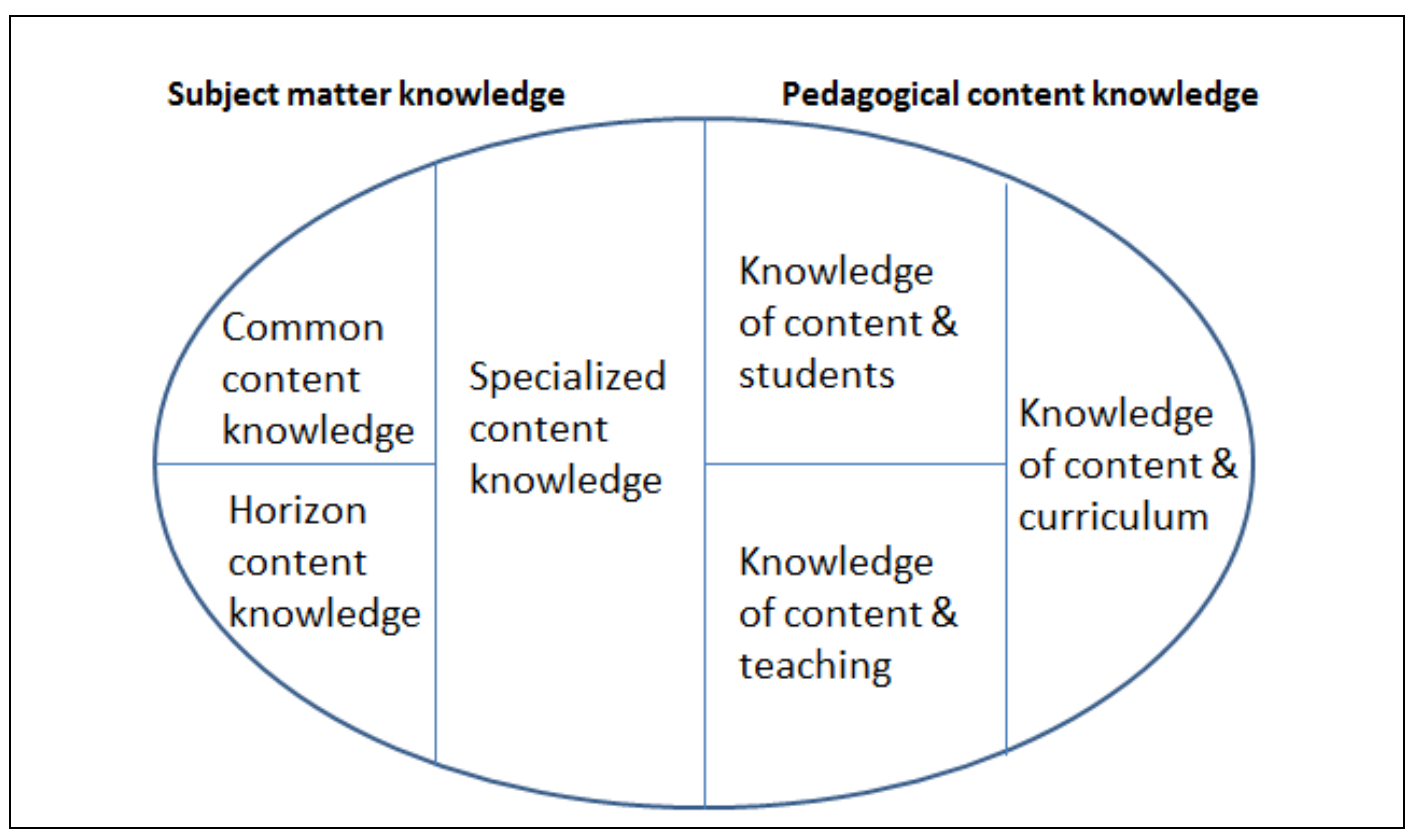

Figure 4-3. Domains of Mathematical Knowledge for Teaching. image adapted from Ball et al. (2008, p.403)

The left half of Figure 4-3 focuses on subject matter knowledge. The first component of subject matter knowledge is common content knowledge, which refers to knowledge that is not specific to teaching, but is common to other settings as well. Examples of common content knowledge include knowing how to perform operations and how to compare fractions and decimals. The second component of subject matter knowledge is specialized content knowledge, which is the content knowledge that is specific to teaching. This includes flexible mathematical knowledge which allows teachers to work with student errors, make sense of nonstandard algorithms, and unpack the mathematical work of others. Ball et al. (2008) distinguish specialized content 
knowledge from the conceptual understanding of content that they would want all students to have. They differentiate that "[w]e do not hold as a goal that every learner should be able to select examples with pedagogically strategic intent, to identify and distinguish the complete range of different situations modeled by $38 \div 4$, or to analyze common errors" (2008, p. 401). Specialized content knowledge is specific to teachers, where engineers or accountants would have different types of specialized mathematical knowledge for their fields. A third component of subject matter knowledge captures the knowledge of how mathematical content develops across grade spans. As an example of this horizon content knowledge, a fourth grade teacher should be aware of how the area model they use for multidigit multiplication relates to the multiplication of fractions and algebraic expressions, as well as relating to division strategies.

Pedagogical content knowledge is the overlap between specific content knowledge (in this case mathematics), and knowledge of teaching and learning. Ball et al. (2008) split pedagogical content knowledge into three components (see the right half of Figure 4-3). Knowledge of content and students is the type of knowledge that allows teachers to anticipate student thinking and make sense of students' informally expressed ideas. Knowledge of content and students centers around common student conceptions and errors. While closely related, knowledge of content and students differs from specialized content knowledge in that specialized content knowledge focuses on teachers' flexible thinking about mathematics while knowledge of content and students focuses on teachers' knowledge of common student conceptions and struggles. A second component of pedagogical content knowledge is knowledge of content and teaching. Knowledge of content and teaching includes knowledge of useful examples, models, and representations 
for teaching particular content. Where knowledge of content and students focuses on student activity, knowledge of content and teaching focuses on teacher activity, including a teacher's ability to select and sequence content within and across lessons. A third category of pedagogical content knowledge is referred to as knowledge of content and curriculum, which refers to knowledge of appropriate instructional materials. Ball et al. note that knowledge of content and curriculum may be consider a part of knowledge of content and teaching and possibly other categories (such as horizon content knowledge), rather than its own category.

Researchers have linked MKT to both student mathematical achievement and the quality of mathematics instruction. Ball et al. (2005) describe a multiple choice test to assess MKT. In a study comparing the MKT for 700 teachers to the standardized test results of their nearly 3000 students, Ball et al. found that teachers' MKT scores significantly predicted student gain scores. Teacher MKT was as significant as student socio-economic status in predicting student gains.

Hill, Umland, Litke, and Kapitula (2012) link MKT and the mathematical quality of instruction (MQI) made available to students. They used an MQI framework to analyze videos of classroom lessons, focusing on the components of richness of mathematics, working with students and mathematics, errors and imprecisions, and student meaning-making. Hill et al. (2012) found that teachers with high scores on an MKT written assessment had higher levels of MQI, while teachers with low MKT scores also had lower levels of MQI. Teacher knowledge of mathematics impacts the types of learning opportunities made available to children. 
Investigating Alternate Numeration Systems

Given the importance of MKT in supporting children's learning, my study focuses on the role that alternate numeration systems can play in supporting the development of MKT in the domain of whole number and operation. I focus on the role of alternate systems within written curricula, leveraging written curricula as a proxy for the learning opportunities made available for PSTs in mathematics content courses (Stein et al., 2007). I focus on two major research questions:

- How are alternate numeration systems leveraged in PST content textbooks?

- What rationales are provided by textbooks for why PSTs should study alternate systems?

I then draw connections between the themes found in the analysis and the components of MKT outlined above.

\section{Methods}

\section{Selection of textbooks}

I selected textbooks with the intention of including some of the most commonly used curricula for preservice teachers, as well an including a range of both newer curricula ( $4^{\text {th }}$ edition or less) and older curricula ( $9^{\text {th }}$ or higher edition). Textbook adoption lists were provided by the publishers for each of the seven selected curricula. According to the publishers, five of the curricula have been adopted by over 150 college and university campuses across the country (Bennett et al., 2012a; Billstein et al., 2012; Long et al., 2012; Musser et al., 2011a; Sowder et al., 2014). The Beckmann (2014) curriculum has been adopted at over 100 schools and the Bassarear (2012c) curriculum at over 50 schools. While the study does not include every available mathematics curricula 
for PSTs, the sample does include textbooks which are in common use across the United

States, with all 50 states represented in the sample.

Table 4-1 provides a list of the selected textbooks, alphabetical by author. In the case where there is a textbook and activity book by the same author set, both are included in the analysis ${ }^{8}$. The textbooks used for review are the Instructor Editions provided by the publishers. The three letter code in the first column of Table 4-1 is used to refer to the curricula in subsequent tables.

Table 4-1. Selected textbooks.

\begin{tabular}{|c|c|c|}
\hline \multicolumn{3}{|c|}{ Mathematics Content Curricula Selected for Inclusion in the Study } \\
\hline Author(s) & Textbook Title(s) & $\begin{array}{l}\text { Edition, Year \& } \\
\text { Publisher }\end{array}$ \\
\hline $\begin{array}{l}\text { Bassarear } \\
\text { (Bas) }\end{array}$ & $\begin{array}{l}\text { Mathematics for Elementary School } \\
\text { Teachers } \\
\text { Explorations: Mathematics for } \\
\text { Elementary School Teachers }\end{array}$ & $\begin{array}{l}5^{\text {th }} \text { Edition, }(2012 \mathrm{c}) \\
\text { Brooks Cole, } \\
\text { Cengage Learning }\end{array}$ \\
\hline $\begin{array}{l}\text { Beckmann } \\
(\mathrm{Bec})\end{array}$ & $\begin{array}{l}\text { Mathematics for Elementary Teachers } \\
\text { with Activity Manual(Beckmann, 2014) }\end{array}$ & $\begin{array}{l}4^{\text {th }} \text { Edition, (2014) } \\
\text { Pearson }\end{array}$ \\
\hline $\begin{array}{l}\text { Bennett, Burton, } \\
\& \text { Nelson } \\
\text { (BBN) }\end{array}$ & $\begin{array}{l}\text { Mathematics for Elementary Teachers: A } \\
\text { Conceptual Approach } \\
\text { Mathematics for Elementary Teachers: } \\
\text { An Activity Approach }\end{array}$ & $\begin{array}{l}9^{\text {th }} \text { Edition, (2012) } \\
\text { McGraw Hill }\end{array}$ \\
\hline $\begin{array}{l}\text { Billstein, } \\
\text { Libeskind, \& Lott } \\
\text { (BLL) }\end{array}$ & $\begin{array}{l}\text { A Problem Solving Approach to } \\
\text { Mathematics for Elementary School } \\
\text { Teachers }\end{array}$ & $\begin{array}{l}11^{\text {th }} \text { Edition, (2012) } \\
\text { Pearson }\end{array}$ \\
\hline $\begin{array}{l}\text { Long, DeTemple, } \\
\& \text { Millman } \\
\text { (LDM) }\end{array}$ & $\begin{array}{l}\text { Mathematical Reasoning for Elementary } \\
\text { Teachers }\end{array}$ & $\begin{array}{l}6^{\text {th }} \text { Edition, (2012), } \\
\text { Addison-Wesley } \\
\text { Pearson }\end{array}$ \\
\hline $\begin{array}{l}\text { Musser, Burger, } \\
\& \text { Peterson } \\
\text { (MBP) }\end{array}$ & $\begin{array}{l}\text { Mathematics for Elementary Teachers: } \\
\text { A Contemporary Approach } \\
\text { Student Activities Manual with } \\
\text { Discussion Questions for the Classroom }\end{array}$ & $\begin{array}{l}\text { 9th }{ }^{\text {th }} \text { Edition, (2011) } \\
\text { Wiley }\end{array}$ \\
\hline $\begin{array}{l}\text { Sowder, Sowder, \& } \\
\text { Nickerson (SSN) }\end{array}$ & $\begin{array}{l}\text { Reconceptualizing Mathematics for } \\
\text { Elementary School Teachers }\end{array}$ & $\begin{array}{l}2^{\text {nd }} \text { Edition, (2014) } \\
\text { Freeman }\end{array}$ \\
\hline
\end{tabular}

\footnotetext{
${ }^{8}$ Two Pearson curricula, Billstein, Libeskind, and Lott (2012) and Long, DeTemple, and Millman (2012), mention activity books created by a different Pearson authoring team (Dolan, Williamson, and Muri). These activity books were not included in the study because of the different authoring team and the minimal inclusion of alternate bases.
} 
Investigating Alternate Numeration Systems

\section{Thematic analysis}

Thematic analysis (Braun \& Clarke, 2006) provides a strategy for using data to generate a framework. I selected thematic analysis to address my research questions because there was no pre-existing framework for classifying ways to leverage alternate bases. Braun and Clarke outline six phases for thematic analysis: "(1) familiarize yourself with the data, (2) generate initial codes, (3) search for themes, (4) review themes, (5) define and name themes, and (6) produce the report" (p. 87). These methods are similar to grounded theory research methods (Creswell, 1998) in that there is a focus on open coding, grouping codes, and selecting and defining overarching themes. Unlike grounded theory, there is not a focus on repeatedly gathering more data with the intention of reaching saturation. Rather, the goal is to find themes within a given body of data, in this case, a set of PST textbooks.

Coding rationales for leveraging alternate bases. I began with two related research questions. How are alternate numeration systems leveraged in PST content courses? And why should PSTs study alternate numeration systems? Within my analysis, I focused on the second of these questions first, examining the rationales that were provided by the curricula. I use the term rationales to refer to justifications that were written in the curricula that addressed why alternate systems were included as a focus of study. Generally, these rationales were located within the introductory or conclusion of textbook sections which relied on alternate systems, or they were attached to specific alternate system examples. I created codes to capture different types of rationales. Next I describe this coding process, following the creation of a rationale code. 
Investigating Alternate Numeration Systems

Consider the following rationale, provided at the beginning of an activity for working with base three using manipulatives.

In the next activity, you will use a common hands-on model, called base pieces, to investigate these concepts. To make the experience more like that of your future students, you will be working in bases other than ten with which you are already familiar. (Musser, Peterson, \& Burger, 2011b, p. 22)

Initially I coded this rationale simply as why study other number systems. As I found more instances where textbooks justified the inclusion of alternate systems, I noticed that the rationales varied. I chose to refine the code why study other number systems to capture the various reasons provided by the curricula. The rationale above focuses on experiencing the type of learning context that children experience, therefore, I coded it experience what children experience.

As a contrast, the following rationale focused more on making sense of algorithms by examining those algorithms in an alternate number system. This rationale appears before an activity on adding and subtracting in base five: "One of the best ways to gain insight into the addition and subtraction algorithms is by thinking the process through using manipulatives that represent other number systems" (Bennett, Burton, \& Nelson, 2012b, p. 70). Due to the focus on algorithms, I chose to code this rationale insight into algorithms or operations.

Table 4-2 shows the codes for rationales for including alternate systems, as identified in the various curricula. Within the table, I have sorted the codes by themes. These themes were created by analyzing across the codes to look for common elements. The first theme gathers together rationales which highlight the benefits of alternate bases for improving understanding base ten. Therefore, I named this theme understanding the 
mathematical structure of base ten. The second theme gathers together rationales related to leveraging alternate bases to help PSTs understand children's learning experiences. I chose to name this theme understanding the learning process of children. Not all rationale codes fit clearly into one of these two themes, so I created a third category for these additional codes.

Table 4-2. Rationales for alternate numeration systems

\begin{tabular}{|l|c|c|c|c|c|c|c|}
\hline Codes for Rationales for Including Alternate Numeration Systems \\
\hline & Bas & BBN & BLL & LDM & MBP & SSN \\
\hline \begin{tabular}{l|c|c|c|c|c|c|c|}
\hline Rationales for understanding the mathematical structure of base ten \\
\begin{tabular}{|l|} 
Appreciate difficulty or power of base \\
ten
\end{tabular} & $\mathrm{X}$ & & $\mathrm{X}$ & $\mathrm{X}$ & & $\mathrm{X}$ \\
\hline To make sense of math structure & $\mathrm{X}$ & $\mathrm{X}$ & & $\mathrm{X}$ & $\mathrm{X}$ & $\mathrm{X}$ \\
\hline Deepen understanding of base ten & $\mathrm{X}$ & & $\mathrm{X}$ & $\mathrm{X}$ & $\mathrm{X}$ & $\mathrm{X}$ \\
\hline Insight into algorithms or operations & & $\mathrm{X}$ & $\mathrm{X}$ & $\mathrm{X}$ & $\mathrm{X}$ & $\mathrm{X}$ \\
\hline Rationales for understanding the learning process of children & & \\
\hline Experience what children experience & $\mathrm{X}$ & & & & $\mathrm{X}$ & $\mathrm{X}$ \\
\hline $\begin{array}{l}\text { Focus on specific challenges for } \\
\text { children }\end{array}$ & $\mathrm{X}$ & & & & $\mathrm{X}$ & $\mathrm{X}$ \\
\hline $\begin{array}{l}\text { Understand children will struggle } \\
\text { (general) }\end{array}$ & $\mathrm{X}$ & & $\mathrm{X}$ & & $\mathrm{X}$ & $\mathrm{X}$ \\
\hline Additional rationales & $\mathrm{X}$ & & & $\mathrm{X}$ & $\mathrm{X}$ & \\
\hline $\begin{array}{l}\text { You may have to or want to teach } \\
\text { alternate systems }\end{array}$ & $\mathrm{X}$ & & & $\mathrm{X}$ & & \\
\hline Other bases are used in the world & & & & \\
\hline
\end{tabular}
\end{tabular}

Coding ways of leveraging alternate bases. After finding themes among the rationales within the curricula, I returned to the curricula to focus on my other research question: How are alternate numeration systems leveraged in PST content courses? This question focuses on the types of alternate system activities and explanations which appeared across the curricula. To address this question, I examined all textbook sections

\footnotetext{
${ }^{9}$ The abbreviations refer to individual curricula. See Table 4-1 to match abbreviations to specific textbooks. Beckmann curriculum is not included because it did not focus on alternate numeration systems.
} 
which focused on whole number and operations and included examples of alternate number systems. I began the analysis by reading through all relevant sections to become familiar with the data. Then all of these sections were scanned to create digital copies. This allowed for the use of qualitative data analysis software (HyperResearch $\left.{ }^{\mathrm{TM}}\right)$ as an organizational tool during the coding process. I broke the data into codeable segments. Examples of segments include paragraphs of explanation, worked examples, and sets of related exercises. Most pages contained between 3 and 8 segments, though some exercise pages contained more segments. Because my intention was to highlight different ways in which alternate bases were leveraged, rather than provide precise counts for how often each activity type occurred, I chose to make segments large enough to capture repeated instances of an activity type. For example, if an exercise section provided six practice problems on translating between bases, these six problems could be captured within a single segment.

The coding process for the textbook sections followed the same process of thematic analysis that was used for the rationales. Thematic analysis was selected because of the absence of an existing framework for classifying ways to leverage alternate bases. Initial codes were developed by creating labels, or codes, for the types of activities and discussions found within the textbooks. As I analyzed more textbook sections, additional codes were added and existing codes refined. To better understand the coding process, consider the segment in Figure 4-4. 


\section{Base six}

The following activities are designed to help you understand base six.

1. a. Take some time, individually or together as a group, to learn how to count in base six.

b. Describe what helped you to accomplish this task.

2. Try the following exercises, both to assess your understanding and to stretch your understanding. Answers are at the end of this section.

What comes after $25_{\text {six }}$ ? after $555_{\text {six }}$ ? after $1235_{\text {six }}$ ?

What comes before $40_{\text {six }}$ ? $300_{\text {six }}$ ? $12340_{\text {six }}$ ?

3. Describe one area in which you had initial difficulty, for example, what comes after 555 ? Describe the difficulty and what helped you get unstuck.

Figure 4-4. PSTs are asked to work within base six to make sense of the base six system (Bassarear, 2012a, p. 43).

The segment in Figure 4-4 contains an activity for making sense of base six. The first feature I highlighted was the focus on counting within a new base and finding the numbers before and after given numbers. I considered creating three separate codes for this idea: counting, finding the number after, and finding the number before. However, because all three of these activities relate to the common task of making sense of adjacent numbers, I chose to combine these three ideas into a single code of within base up or down by one. This code captures the essence of counting up or down within a number system by focusing on adjacent numbers. This code was also applied to exercises which asked PSTs to name the next several numbers after a given number, based on the hypothesis that PSTs would count up one number at a time.

A second feature I noticed within the above segment was the focus on PST reflection. The PSTs are asked to describe what struggles they had, and what helped them to overcome their struggles. I created the code reflecting on learning a number system to capture this idea of moving beyond solving the mathematical task and focusing on thinking about the learning process. The third code I attached to this segment is 
modern alternate system-base 6 . In order to understand which alternate number systems were in common use in curricula, I used a collection of codes with names of specific systems (such as Babylonian, Roman, or binary) as well as two general names, historic - other and modern alternate system, which were annotated to indicate which system was being used. I use the term modern alternate system to capture alternate systems which use symbols and format similar to base ten.

As described above, a given segment could receive multiple codes, capturing a variety of aspects of the segment. In addition to the codes for counting, reflecting, and naming the system, the segment in Figure 4-4 also received the code PST investigates an unexplained system, highlighting the fact that this activity was not preceded by a textbook explanation of base six.

Table 4-3 displays some of the codes ${ }^{10}$ that were developed to describe segments across the curricula. The codes within Table 4-3 are placed in categories. I created these categories as a part of the thematic analysis process by examining commonalities between codes and looking for overarching roles for alternate systems across the curricula. One distinction that I noticed among the codes was that some codes involved working within a single system while others involved working across multiple systems. While this distinction may appear minor at first, further examination of this dichotomy revealed a connection between this dichotomy and the two primary rationales for studying alternate bases (understanding the structure of base ten and understanding children's experiences). Working within a single number system more closely parallels

\footnotetext{
${ }^{10}$ Additional codes not included in Table 4-3 include codes for names of number systems (e.g. Roman, binary) and types of visual models (e.g. base pieces). The full set of codes is available in the Appendix.
} 
Investigating Alternate Numeration Systems

how children learn about base ten, while working across multiple number systems creates opportunities for comparing the underlying structure of those number systems. The two dominant ways of leveraging alternate systems are discussed further in the results and discussion section, along with an elaboration of the connection between these ways of leveraging alternate systems and the two primary rationales for studying alternate bases.

Table 4-3. Codes for analysis of alternate numeration systems

\begin{tabular}{|l|c|c|c|c|c|c|}
\hline Codes for the Use of Alternate Bases in Narratives, Activities, and Exercises \\
\hline & \multicolumn{6}{|c|}{ Curricula ${ }^{11}$} \\
\hline & Bas & BBN & BLL & LDM & MBP & SSN \\
\hline
\end{tabular}

Comparison of multiple numeration systems

\begin{tabular}{l|c|c|c|c|c|c|}
\hline Translate OUT OF base ten & $\mathrm{X}$ & $\mathrm{X}$ & $\mathrm{X}$ & $\mathrm{X}$ & $\mathrm{X}$ & $\mathrm{X}$ \\
\hline Translate TO base ten & $\mathrm{X}$ & $\mathrm{X}$ & $\mathrm{X}$ & $\mathrm{X}$ & $\mathrm{X}$ & $\mathrm{X}$ \\
\hline Comparing number systems & $\mathrm{X}$ & $\mathrm{X}$ & $\mathrm{X}$ & $\mathrm{X}$ & $\mathrm{X}$ & $\mathrm{X}$ \\
\hline Describing advantages or disadvantages & $\mathrm{X}$ & $\mathrm{X}$ & $\mathrm{X}$ & $\mathrm{X}$ & $\mathrm{X}$ & $\mathrm{X}$ \\
\hline Connecting to a standard algorithm & $\mathrm{X}$ & $\mathrm{X}$ & $\mathrm{X}$ & $\mathrm{X}$ & $\mathrm{X}$ & $\mathrm{X}$ \\
\hline General statements about number systems & $\mathrm{X}$ & $\mathrm{X}$ & $\mathrm{X}$ & $\mathrm{X}$ & $\mathrm{X}$ & $\mathrm{X}$ \\
\hline
\end{tabular}

Immersion within a numeration system

\begin{tabular}{|l|c|c|c|c|c|c|}
\hline Within base operations & $\mathrm{X}$ & $\mathrm{X}$ & $\mathrm{X}$ & $\mathrm{X}$ & $\mathrm{X}$ & $\mathrm{X}$ \\
\hline Within base up or down by one & $\mathrm{X}$ & & $\mathrm{X}$ & & $\mathrm{X}$ & $\mathrm{X}$ \\
\hline Looking for patterns within a system & $\mathrm{X}$ & $\mathrm{X}$ & & $\mathrm{X}$ & $\mathrm{X}$ & \\
\hline $\begin{array}{l}\text { PSTs Investigate an unexplained system } \\
\text { PSTs invent a system }\end{array}$ & $\mathrm{X}$ & $\mathrm{X}$ & $\mathrm{X}$ & $\mathrm{X}$ & $\mathrm{X}$ & $\mathrm{X}$ \\
\hline PSTs invent an algorithm/procedure & $\mathrm{X}$ & $\mathrm{X}$ & & & $\mathrm{X}$ & \\
\hline Reflecting on learning a number system & $\mathrm{X}$ & $\mathrm{X}$ & & $\mathrm{X}$ & & \\
\hline $\begin{array}{l}\text { Other types of activities } \\
\text { Focusing on zero }\end{array}$ & $\mathrm{X}$ & $\mathrm{X}$ & $\mathrm{X}$ & $\mathrm{X}$ & $\mathrm{X}$ & $\mathrm{X}$ \\
$\begin{array}{l}\text { Working with alternate system decimals / } \\
\text { fractions }\end{array}$ & & & & & $\mathrm{X}$ \\
\hline $\begin{array}{l}\text { Creating arguments for or against alternate } \\
\text { bases }\end{array}$ & & $\mathrm{X}$ & & & $\mathrm{X}$ & \\
\hline $\begin{array}{l}\text { Working with operation tables } \\
\text { Non-routine / other }\end{array}$ & $\mathrm{X}$ & & $\mathrm{X}$ & $\mathrm{X}$ & $\mathrm{X}$ & $\mathrm{X}$ \\
\hline
\end{tabular}

\footnotetext{
${ }^{11}$ The abbreviations refer to individual curricula. See Table 4-1 to match abbreviations to specific textbooks. Beckmann curriculum is not included because it did not focus on alternate numeration systems.
} 


\section{Results \& Discussion}

In the results section I argue that within the selected curricula there were two overarching rationales for studying alternate numeration systems (understanding the mathematical structure of base ten and understanding the learning process of children). I also argue that the curricula activities related to alternate numeration systems can be split into two major categories. Before discussing these results, I describe the inclusion of alternate systems within the seven selected curricula.

Of the seven curricula included in this study, six contained a focus on alternate numeration systems within the chapters on whole numbers and operations. The seventh curriculum, Beckmann (2014), stated that the textbook does not focus on alternate bases explicitly. However, Beckmann pointed to a few exercises that allow PSTs to explore the importance of regrouping by considering contexts where regrouping occurs at values other than ten. These examples included mixed units of feet and inches and regrouping by 60 for the context of minutes and hours. Beckmann argued that these activities "allow students to grapple with the significance of the base in place value without getting bogged down in the mechanics of arithmetic in other bases" (p. xiii). I exclude the Beckmann curriculum from the remainder of the results section because of its limited use of alternate systems.

Across the six curricula which contained a focus on alternate bases, both historic and modern alternate numeration systems were used. Historic numeration systems tended to be introduced first, building up from additive to place value systems. While historic systems appeared primarily in sections on numeration (i.e. sections on the 
structure of number systems), modern alternate bases appeared in both sections on numeration and sections on operations with whole numbers.

The remainder of the results and discussion section is split into two major sections. First I focus on themes within the rationales provided by the curricula, highlighting and comparing two major themes which were common to most of the curricula. Then I focus on the types of activities provided by the curricula, presenting examples from across the collection of curricula to create a rich description of each of the two primary ways in which alternate numeration systems are leveraged. This is followed by a discussion of the affordances of each approach and a connection to the MKT framework.

\section{Themes within the textbook rationales}

Each of the selected curricula provided rationales for including alternate numeration systems. Often appearing at the beginning or end of a section on alternate numeration systems, these rationales provide PSTs with insight into why alternate numeration systems appear in the textbooks. As discussed in the methods section, there were two overarching themes for the types of rationales offered by textbooks: understanding the mathematical structure of base ten and understanding the learning process of children. In this section I focus on each of these rationales, providing insight into how they appeared across the curricula.

Rationales focused on mathematical structure. "One very useful application of bases other than base ten is as a way to deepen our understanding of number systems, arithmetic in general, and our own decimal system" (Long et al., 2012, p. 138). 
One of the two overarching rationale themes for the study of alternate numeration systems focused on the benefits of better understanding base ten by comparing base ten with other number systems. These rationales hinged around the idea that comparing between number systems provides PSTs with an opportunity to step back from working within a single number system (base ten) and begin to see base ten as an example of a wider phenomenon. All six of the curricula that included alternate bases provided explicit rationales about the advantages of leveraging multiple number systems to make sense of mathematical structure or deepen understanding of base ten. In this section I highlight two types of rationales provided by the curricula for increasing understanding of base ten: rationales related to deepening understanding of the mathematical structure of place value systems and rationales that focus on making sense of standard algorithms by placing them in alternate systems.

Alternate numeration systems can be leveraged to make sense of the mathematical structure of numbers systems in general and to deepen understanding of base ten in particular.

The primary purpose of this exploration is to deepen your understanding of base ten - this is, your understanding of base, place value, and the role of zero. The structures of a system are often best seen by putting the familiar in an unfamiliar context. (Bassarear, 2012a, p. 43)

Bassarear's rationale connected both to increasing the overall understanding of base ten and to specific examples of structural components of number systems (base, place value, and zero) which could be investigated through alternate bases. This reasoning captured the value of alternate systems as a tool to take routine or familiar content and develop a deeper understanding by shifting to a non-routine context. For example, the ten to one 
relationship between place values is so commonplace that PSTs may give the relationship little thought. However, changing the relationship, by shifting to a different base, may draw attention to the underlying structure of place value systems.

In addition to focusing on the structure of number systems, the rationales related to understanding base ten also included a focus on standard algorithms, particularly by comparing the algorithms across bases. "One of the best ways to gain insight into the addition and subtraction algorithms is by thinking the process through using manipulatives that represent other number systems" (Bennett et al., 2012b, p. 70). This rationale focused specifically on the advantages of alternate base pieces to support PSTs in unpacking the meaning of standard algorithms. This line of reasoning, captured in the code insight into algorithms, appeared in five ${ }^{12}$ of the curricula (Table 4-2). Long et al. (2012) highlighted this focus on algorithms while also connecting to making sense of place value.

The algorithms for addition and subtraction are just as valid in base five, or any other base, as they are in base ten... Our approach, therefore, is to consider other bases right along with base ten. By studying bases together this way, you gain a greater understanding of the whole idea of positional notation and the related algorithms. (p. 150)

Because standard algorithms are based on place value structure, making sense of standard algorithms can serve as an activity that promotes understanding of underlying structure as well as understanding of algorithms. The prior rationale highlighted both of these purposes. The opportunity to support making sense of standard algorithms is particularly important in light of research that highlights PST and teacher struggle to explain the

\footnotetext{
${ }^{12}$ While explicit rationales for gaining insight into standard algorithms appear in five of the curricula, all six curricula included examples of standard algorithms in alternate bases. This hints at an implicit rationale for insight into algorithms across all the curricula in the sample.
} 
Investigating Alternate Numeration Systems

mathematics that underlies common algorithms (J.-J. Lo et al., 2008; Ma, 1999;

Thanheiser, 2009a). Shifting algorithms to alternate systems can create a context for unpacking the otherwise routine algorithms.

While the first theme for rationales focused on the goal of improving PSTs' grasp of the mathematical structure of base ten, the second theme highlights the role of alternate systems in prompting PSTs to consider the learning process of children.

Rationales focused on children's thinking. "I wish I had a dollar for every student who has said something like 'Wow, no wonder it's hard for little kids to learn how to count; I never thought of it [our system] that way before" (Bassarear, 2012c, p. 102). This rationale captured one common justification provided for including alternate bases in PST textbooks. Learning an alternate system creates an opportunity for PSTs to experience the learning process for making sense of a number system and to appreciate the difficulty of that process. While all six curricula included rationales about improving understanding of base ten, four of the curricula also included explicit rationales for understanding the learning processes of children. These rationales included general statements about understanding that children will need time to make sense of base ten. For example, one rationale for including alternate bases stated that "the intent here is to introduce you to different bases so that you have a better understanding of our own baseten system, and you understand why children need time to learn to operate in base ten" (Sowder et al., 2014, p. 35). This line of reasoning implies that learning alternate systems helps PSTs to develop empathy for their students. Note that the first half of this rationale focuses on understanding base ten, while the second half focuses on children. It 
was not uncommon for curricula to combine both types of rationales within a single argument for including alternate bases.

In addition to this general argument for understanding that children struggle, some rationales focused on more specific mathematical challenges. A specific example related to counting is provided in the section on immersion activities. PSTs were reminded "The goal here is 'sense making' as opposed to 'just do it.' The more you get into this role play, the better you will understand the difficulties young children have when learning to count in our numeration system!” (Bassarear, 2012a, p. 38). This rationale focused on experiencing (i.e., role playing) the type of experience that children will encounter. The rationale's warning about trying to make sense of the mathematics, rather than just find the correct answer, implies that the ways in which PSTs engage with alternate bases affect the types of learning opportunity that alternate bases afford.

The intention of highlighting two themes among the rationales is not to imply that each curriculum leveraged only one type of rationale and ignored the other. Rather, the intention is to highlight two arguments used by textbooks to promote PSTs' engagement with the novel context of alternate bases. Across the collection of six curricula which focused on alternate bases, all six used arguments about improving understanding of base ten and four of the curricula also argued for understanding the learning processes of children. The novel context of alternate numeration systems can lend itself to both types of learning opportunities.

In the next section of the results, I focus on the types of mathematical activities and explanations provided across the six curricula. These activities inform the types of learning opportunities made available to PSTs. 
Investigating Alternate Numeration Systems

\section{Themes within the activities \& explanations}

As described in the methods section, the narratives, activities, and exercises related to alternate systems were coded using the codes in Table 4-3. By analyzing these codes, I determined that there were two overarching themes among the codes, corresponding with whether the code involved one or multiple numeration systems. I chose the name comparison between systems to classify codes which involved multiple systems, such as translating to base ten, comparing systems, and describing advantages and disadvantages. I selected this name to capture the idea that students have the opportunity to consider a system other than base ten in order to better understand base ten. I chose the name immersion within a system to classify codes which involved working within a single system, such as within base operations, PSTs invent a system, and reflecting on learning a number system. I selected the term immersion to draw attention to the idea that PSTs are working within a system, experiencing a learning process similar to that of children learning base ten. Next I provide rich descriptions of each of these overarching themes.

Activities related to comparison. Alternate numeration systems serve a particularly important role in making sense of place value systems due to the ways in which the different number systems embody variation in structure. In her book on variation theory, Lo (2012) captures this idea, stating that "to discern previously taken for granted features of familiar situations, learners must experience for themselves certain patterns of variation and invariance of these features" (p. 83). Alternate numeration systems provide an opportunity to vary key features of the familiar base ten system. Historic systems provide the chance to vary between additive and place value structures. 
For example, to record three ones in the Roman system one would record III, while three hundreds would be CCC. However, in base ten, the symbol 3 can indicate either three or three hundred, depending on its location (i.e. 303). Likewise, alternate base systems keep the place value structure invariant, but allow the value of the base to vary, drawing attention to the quantity needed for regrouping.

In this section I provide examples of two distinct ways in which the comparison of alternate numeration systems appeared in the curricula. First I focus on comparing the structures of different number systems, highlighting the use of historic number systems as examples for comparison. Then I discuss the role of converting between number systems as a way to focus on place value structure.

Comparing systems with different structure. In order to better understand a mathematical structure, such as the base ten number system, it can be helpful to consider ways to vary key aspects of the structure (M. L. Lo, 2012; Thanheiser, 2015b). All six of the curricula that leverage alternate numeration systems included examples of comparing the different structures of those alternate systems to base ten. Five ${ }^{13}$ of the six textbooks included a numeration section which introduced tallying, Egyptian, Roman, Babylonian, and Mayan numerations systems as the primary historic examples. Some of the texts supplied an historic backdrop describing the location and time period where the number system was used, while others dove straight into the mathematics. While learning history for the sake of understanding the cultural development of mathematics is a worthy goal, I found that it was not a central focus of these textbook sections on historic systems.

\footnotetext{
${ }^{13}$ Sowder, Sowder, and Nickerson (2014) contains Roman numerals but does not focus on the other major historic systems.
} 
Rather, the goal across curricula appeared to be to compare the underlying structure of each system and point out advantages of some systems over others.

Historic systems were leveraged to describe different ways in which number systems can be structured. For example, Musser et al.(2011a) used a comparison between tallying and the Egyptian system to introduce the term additive system. This refers to numeration systems where the value of a number is found by summing the value of different symbols. Then Musser et al. drew a comparison between Babylonian and Egyptian, introducing the language of place value to describe how the location of a symbol allows the symbol to take on different values. As each ancient number system was introduced, it was compared to other systems, building a repertoire of different structures to draw upon. Curricula used these differences as opportunities to introduce formal language about place value and to discuss the significance of zero. A textbook comparison cross five historic systems is provided in Figure 4-5.

\begin{tabular}{|c|c|c|c|c|c|c|}
\hline SYSTEM & ADDITIVE & SUBTRACTIVE & MULTIPLICATIVE & POSITIONAL & $\begin{array}{l}\text { PLACE } \\
\text { VALUE }\end{array}$ & $\begin{array}{l}\text { HAS A } \\
\text { ZERO }\end{array}$ \\
\hline Tally & Yes & No & No & No & No & No \\
\hline Egyptian & Yes & No & No & No & No & No \\
\hline Roman & Yes & Yes & Yes & Yes & No & No \\
\hline Babylonian & Yes & No & Yes & Yes & Yes & No \\
\hline Mayan & Yes & No & Yes & Yes & Yes & Yes \\
\hline
\end{tabular}

Figure 4-5. Five historic systems were compared in a single chart (Musser et al., 2011, p. 66).

While comparisons to the Hindu-Arabic system do not appear in Figure 4-5, each curriculum did make explicit comparisons between our Hindu-Arabic system and historic systems. In these comparisons, the virtues (and sophistication/ difficulty) of base ten were highlighted by comparing the base ten place value system to other systems with 
different structure. Bassarear (2012c) concluded a section on historic systems by summarizing the advantages of an historic overview of the development of numeration systems.

We have explored different counting systems to give you an appreciation of the significance of base ten and its abstractness - it took humans many thousands of years finally to invent such a powerful numeration system. In the course of working on the explorations, you have come to appreciate the importance of mathematical vocabulary, including the terms digit and place. (2012c, p. 102)

Bassarear highlighted (a) the importance of base ten and its position as a sophisticated number system, and (b) the use of alternate systems to draw attention to the structure of base ten. The focus on comparing historic numeration systems played an important role across all six of the textbooks, providing a collection of authentic examples of different ways to structure number systems.

Translating between systems. Translating between number systems was one of the most common alternate numeration system activities across the curricula, occurring with both historic and modern alternate systems. During the coding process, the code translate out of base ten was used 97 times and the code translate to base ten was used 91 times $^{14}$. All six curricula which leveraged alternate systems included more than ten examples or exercises of converting between bases. The sheer prevalence of translating between systems led me to consider its importance as a central activity in comparing number systems.

\footnotetext{
${ }^{14}$ Because segments could include multiple instances of an idea, such as translating, these numbers do not indicate precise counts. The numbers are included to highlight the frequent occurrence of translating as an activity.
} 
Translating between bases focuses PSTs' attention on the underlying structure of the alternate base and how that structure differs from base ten. When determining how many of each place value are needed, the PSTs have the opportunity to unpack the meaning of each place value. The procedure demonstrated in Figure 4-6 provides a way to determine the number of groups for each power of five, highlighting the place values of $625,125,25,5$, and 1 in base five. In some texts, new number systems were introduced by demonstrating how to convert between base ten and the new systems. While most curricula provided algorithms for how to translate between systems (see Figure 4-6), some curricula provided an opportunity for PSTs to invent their own strategy for efficiently translating between systems.

Task: Convert 824 to base five.

$$
\begin{aligned}
& 5^{4}=625 \rightarrow \underline{625}{ }_{-625}^{824}\lfloor 1 \quad \text { How many groups of } 625 \text { in } 824 ? \\
& 5^{3}=125 \rightarrow \underline{125} \begin{array}{r}
199 \\
-125
\end{array} \quad \text { How many groups of } 125 \text { in } 199 \text { ? } \\
& 5^{2}=25 \rightarrow 25 \quad \begin{array}{r}
74 \\
-50
\end{array} \quad \text { How many groups of } 25 \text { in } 74 \text { ? } \\
& 5^{1}=5 \rightarrow \begin{array}{r}
5 \\
\quad 20
\end{array} \quad \begin{array}{r}
4 \\
-20
\end{array} \text { How many groups of } 5 \text { in } 24 \text { ? } \\
& 5^{0}=1 \rightarrow \frac{1}{4} 4 \quad \text { How many } 1 \mathrm{~s} \text { in } 4 \text { ? } \\
& -4
\end{aligned}
$$

Thus, $824=11244_{\text {five }}$.

Figure 4-6. An example of comparing between numeration systems by focusing on converting. In this example, 824 is converted to base five (Billstein et al., 2012, p.61).

Translating between systems provided an opportunity to focus on the meaning of place value, but this is an opportunity, not a guarantee. Bassarear (2012a) warned that "[i]t is important to note that the goal of this [translating] is not to 'get' the 'procedure' for translating from one base to another but rather ... to deepen your understanding of 
base ten, which is the base you will 'teach' to your future students" (p. 44). Toward this goal, Bassarear included an explicit prompt for reflecting on the learning process:

"Describe the most important learnings from translating to and from base ten. In each case, first describe what it was that you learned and then describe how you learned it" (Bassarear, 2012a, p. 45). In order to make the most of the translating tasks, PSTs must step back and reflect on their own learning. Three of the curricula include specific prompts for PSTs to reflect on their learning process.

Each of the six curricula that used alternate numeration systems included multiple ways of engaging in comparison of systems, including translating between systems and explicitly comparing underlying structures of systems. Next I discuss activities that leveraged an immersion approach to alternate numeration systems.

Activities related to immersion. Immersing within an alternate system provides an opportunity for PSTs to consider the mathematical ideas needed to make sense of a number system and to reflect on the types of activities young children will encounter when learning base ten. I provide two examples of this type of activity, with the first focusing on counting in base six and the second focusing on adding within base four.

Consider the example in Figure 4-4. PSTs were asked to begin the activity by counting within a new number system. Then PSTs were asked to consider what number occurs before and after particular transitions in the counting process. For example, what number occurs just after $25_{\text {six }}$ ? These transitions mirror the base ten transitions that young children also struggle with. "For example, for little children, remembering the number after 29 is more difficult than remembering the number after 28 " (Bassarear, 2012b, p. 113). Alternate bases provide an opportunity for PSTs to experience the same 
Investigating Alternate Numeration Systems

difficulties their students may experience and then to step back and reflect on those difficulties (see prompt 3 in Figure 4-4). Counting activities in alternate systems were included within four of the curricula.

The most frequently used code across all of the curricula was within base operations. This code appeared in all six curricula and captures instances of addition, subtraction, multiplication, division, and ordering which occur within an alternate system. An example of a within base operation is provided in Figure 4-7. The operation of combining two base four numbers was illustrated using base four blocks. The text highlighted the need to regroup whenever four or more of one object was found. Rather than relying on a standard vertical algorithm for addition, this example modeled an approach that might be used by a young child who was developing a sense-making strategy for multidigit addition.

Suppose we want to add $231_{\text {four }}$ and $311_{\text {four }}$ using base-four blocks, with the small block as the unit. We could first express the problem as

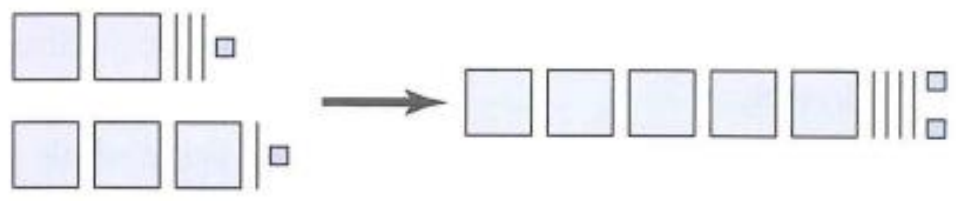

We have too many longs (in base four), so trade four longs for a flat. Now we have too many flats (each representing four squared). Trade four flats for a large cube (which represents four cubed):
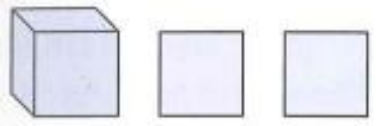
Represents the answer, which is $1202_{\text {four }}$. The blocks represent 1 four cubed, 2 fours squared, and 2 ones.

Figure 4-7. An example of a within base operation: adding two numbers in base four (Sowder et al., 2014, p. 37).

The curricula provided opportunities for PSTs to gain experience using manipulatives to make sense of place value and operations, allowing the PSTs to come to 
value tools they may use in their own teaching practice. All six of the curricula that focus on alternate bases used the visual model of base pieces (also called Dienes blocks) for performing operations within modern alternate base systems. Some of the curricula explicitly highlighted the advantages of base pieces in making sense of multidigit operations and regrouping, drawing attention to the impact of manipulatives when supporting school children. "Multibase blocks are manipulatives that have proven to be extremely useful in coming to understand any base system, but primarily base ten in elementary school" (Sowder et al., 2014, p. 30). PSTs have the opportunity to experience the utility of manipulatives as they engage in the same types of operation activities their student will engage in.

Trends across the curricula. Looking across the six curricula, both comparison and immersion approaches were common to each curriculum. As shown in Table 4-3, all six curricula provided similar opportunities for PSTs to engage in translation and comparison activities and to consider the advantages of different numeration systems. Each curriculum provided opportunities to consider standard algorithms in alternate systems. These activities can be linked to the textbook rationales for leveraging alternate systems to improve understanding of mathematical structure related to place value systems and algorithms.

While all six curricula contained some activities involving immersion within a system, these activities varied among curricula, as seen in Table 4-3. All curricula included operations within an alternate base, but only three of the curricula provided activities for inventing a numeration system, with the intensity of invention activities ranging from a single exercise in one textbook to multiple pages of invention related 
activities in another. Four of the curricula included counting activities in an alternate system. These four curricula were the same ones that included explicit rationales for understanding the learning process of children (Table 4-2). Determining the number before or after a give number is a basic and important step in making sense of the structure of a number system, for both children and PSTs.

In the next section I draw connections between the MKT framework and the comparison and immersion approaches to alternate numeration systems.

Discussion \& Connections to MKT. A natural extension of thinking about the roles of alternate systems in PST courses is to consider how they align with the mathematical knowledge goals for these students. For each of the overarching roles for alternate systems, I discuss how those roles connect to different components of MKT. First I link the comparison approach to alternate bases to the left half of the MKT framework (subject matter knowledge). Then I link the immersion approach to components on both sides of the MKT framework.

Comparison. Comparing different number systems provides an opportunity for PSTs to develop both common content knowledge and specialized content knowledge. For example, PSTs have the opportunity to improve their common content knowledge by advancing their understanding of place value and standard algorithms. They can develop specialized content knowledge by learning how to unpack place value explicitly and visually. PSTs have opportunities to compare the base ten system to systems without a place value structure and systems with a different base quantity. Stepping back from base ten allows PSTs to see base ten as an example of a wider phenomenon of place value number systems. The third category of subject matter knowledge, horizon content 
knowledge, was not a common focus of the curricula, but there were brief instances

where textbooks began to connect algebraic thinking to the idea of generalizing place value concepts. For example, some textbooks mentioned a generic base b, with place values based on powers of the base. It is possible to make sense of base ten as an example of a wider phenomenon because of the ways in which alternate systems vary key components of base ten.

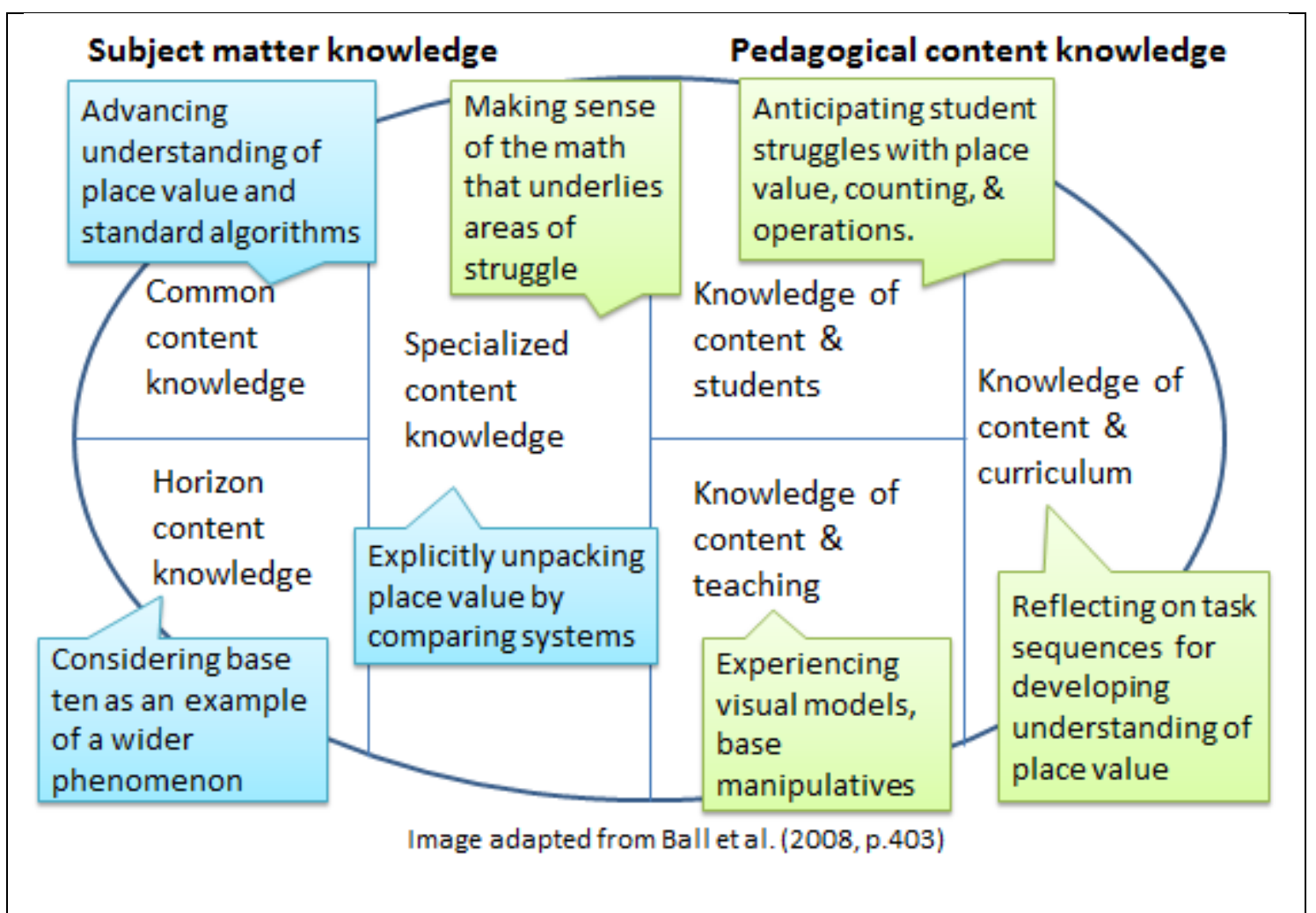

Figure 4-8. Connecting alternate base activities with the MKT framework. The blue boxes relate to comparison activities and the green boxes relate to immersion activities.

Immersion. While the comparison approach to alternate systems lends itself well to supporting a focus on mathematical structure (an aspect of subject matter knowledge), the immersion approach creates an opportunity to focus on both pedagogical content knowledge and subject matter knowledge. Immersion within an alternate system can 
support a dual focus on mathematical structure and how children make sense of a number system.

The immersion approach to alternate numeration systems provides an opportunity for PSTs to be placed in the position of re-encountering fundamental ideas related to number and counting. Moreover, the novel contexts can support PSTs in engaging with these ideas as genuine problems. In all of my teaching experience, I have not encountered a PST who did not immediately know that the number that followed 29 is 30 . But engaging with an alternate numeration system allows PSTs to struggle with a similar task of finding the number that occurs after $25_{\text {six }}$. For example, PSTs may initially create the number $26_{\text {six }}$ to follow the number $25_{\text {six }}$, matching how a child might count twenty-eight, twenty-nine, twenty-ten. By struggling with this issue, and then making sense of the mathematics that underlies this issue, PSTs have the opportunity develop several types of MKT related to whole numbers (see Figure 4-8). First, PSTs develop knowledge of content and students by becoming aware of a common student issue related to learning to count. Second, PSTs develop specialized content knowledge by making sense of the mathematics that causes this struggle (that regrouping occurs in base six after collecting six of one type of unit). Third, PSTs develop knowledge of content and teaching by learning about tools, such as base pieces, which can support children in making sense of the structure of place value systems.

My intention behind linking components of MKT to activities with alternate bases is to highlight the variety of ways in which alternate systems can support developing MKT. In addition to the opportunities found within the curricula, I hypothesize that there are additional ways to leverage alternate systems to promote PSTs' 
developing MKT. For example, the curricula provide opportunities for PSTs to experience tasks and task sequences which support developing understanding of a number system. This could provide a context for reflecting on tasks that support children's learning, a key component of knowledge of content and curriculum. While this was not a primary focus within the set of curricula investigated within this study, this type of reflection is a natural extension of the activities that are provided and could be supported by teacher educators as they enact the curriculum. McClain (2003) found that when PSTs were given opportunities to reflect back on their own learning process after participating in an alternate base task sequence, they were able to distinguish between questions which focused on understanding concepts of place value and those that simply focused on finding correct answers. Moreover, they came to value the conceptual questions over the procedural ones.

\section{Conclusions}

\section{Why alternate numeration systems?}

Regardless of whether ancient number systems and alternate bases are a part of elementary school mathematics, these topics can serve a vital purpose in PST content courses. These non-routine contexts can allow PSTs to step back from their prior knowledge of base ten and begin to see base ten as an example of a wider phenomenon. The alternate numerations systems can be leveraged to encourage PSTs to examine the underlying structure of place value number systems. This opportunity to investigate a novel context may also help PSTs reflect on the process of learning about number systems, a process they will be asked to support in their own elementary classrooms. Alternate numeration systems provide opportunities for PSTs to connect their own 
Investigating Alternate Numeration Systems

struggles in making sense of a new number system to the struggles of their future students, while also learning about strategies, representations, and tasks to overcome those challenges.

\section{Content and pedagogy}

Alternate numeration systems are leveraged by curricula to provide a variety of ways to support PSTs in developing MKT in the content domain of whole number and operation. This study found two dominant roles for alternate bases within the curricula, comparison and immersion, and two overarching types of rationales offered by the curricula. The first rationale focused on improving understanding of mathematical structure, while the second had a more pedagogical intention of understanding how children learn. Each individual curriculum approaches these purposes with different intensities. Sometimes mathematical structure was the primary focus, while pedagogy received a lighter touch. Given the potential benefits of reflecting on the challenges of learning a numeration system, it may be worthwhile for teacher educators and curriculum designers to create additional opportunities for PST reflection. This reflection could focus on (a) common struggles (faced by children and by PSTs) in making sense of numeration systems, (b) the mathematics that underlies these struggles, and (c) tools for overcoming those struggles. Alternate bases provide a special opportunity for this type of pedagogical reflection because the content is both novel to PSTs and structurally similar to the content for children.

Teacher educators may want to take into consideration the intentions of focusing on mathematical structure and pedagogy when selecting a curriculum or choosing and sequencing tasks within a curriculum. When shifting from written to enacted curriculum, 
Investigating Alternate Numeration Systems

teacher educators have the opportunity to underline or undermine intended purposes of the curriculum. Awareness of these purposes can serve as a key piece of teacher educator knowledge.

\section{Further study}

This study provided an analysis of written curricula related to alternate numeration systems, finding two dominant roles for alternate systems across the written materials. While written curricula provides insight into enacted curricula, the enacted curricula is influenced by teacher beliefs and goals (Stein et al., 2007). In order to develop a more clear understanding of the mathematical opportunities made available to PSTs, it would be beneficial to study the curricula as it is enacted in PST mathematics courses across the country. Such a study may provide additional insight into affordances and constraints of leveraging alternate numeration systems for work with PSTs. 


\title{
Chapter 5 : Co-emergence of Models
}

\author{
Reinventing Multidigit Multiplication in Base Five: \\ Making Sense of Co-emerging Models for Meaning and Strategy
}

\begin{abstract}
Preservice teachers (PSTs) know how to perform multidigit multiplication, but they may struggle to unpack the mathematics that underlies the standard algorithm. I used design heuristics from Realistic Mathematics Education to create a task sequence to support PSTs in reinventing a general algorithm for multiplication in base five. I conducted two cycles of a teaching experiment and analyzed student mathematical activity as they engaged with the task sequence. I found that the PSTs were able to reinvent a general partial products strategy for multiplication and that this partial products model co-emerged alongside the PSTs developing model for the meaning of multiplication. I put forth co-emergence of models as a design heuristic for both understanding and advancing student mathematical activity.
\end{abstract}

The purpose of this report is to introduce a design heuristic for developing and analyzing task sequences which support the co-development of multiple interrelated mathematical models. I name the design heuristic co-emergence of models to refer to the idea of two related mathematical models being developed in conjunction with one another (this could also be conceived of as two co-emerging aspects within an overarching model). This design heuristic is considered as an elaboration of the existing emergent models design heuristic (Gravemeijer, 1999). I discuss co-emergence of models within the context of a teaching experiment focused on supporting preservice teachers (PSTs) in developing a conceptual understanding of multidigit multiplication. The focus of this paper is two-fold. First, I argue that PSTs were able to reinvent a model for multiplication which included two co-developing sub-models. One model centered around the meaning of multiplication and the other model centered around strategies for 
performing multiplication. Second, I argue that it is advantageous to focus explicit attention on the interplay of the two co-emerging models, both for task design and for analysis of student mathematical activity. In this way, the co-emergence design heuristic offers a refinement to the existing heuristic of emergent models.

This report is situated in the context of a teaching experiment which leveraged a local instructional theory (LIT) for multiplication for work with preservice teachers (PSTs). In addition to introducing the design heuristic of co-emergence of models, this report also contributes to the body of research on how preservice teachers (PSTs) learn about whole number and operation. Researchers have shown that PSTs lack sufficient mathematical knowledge for teaching in the domain of whole number and operation (Ball, 1990b; Ball et al., 2005; Ma, 1999; Thanheiser, 2009a). While PSTs are able to perform standard algorithms, they struggle to explain the reasoning behind those algorithms (Ball, 1990b; J.-J. Lo et al., 2008; Ma, 1999). How can PSTs support children's learning about number and operation if they themselves struggle to articulate the underlying mathematics? The multiplication task sequence in this report is based on a conjectured local instructional theory for supporting PSTs in developing a richer understanding of the mathematics involved in whole number operations.

\section{Literature Review}

Research indicates that PSTs and teachers often have insufficient mathematical knowledge of the topics they are expected to teach (Ball, 1988; Ma, 1999; Thanheiser, 2009a). For example, when working with standard algorithms for multidigit operations, PSTs may not be able to go beyond finding a numeric answer to justifying why an algorithm works (Graeber, 1999; J.-J. Lo et al., 2008; Philipp et al., 2007) When PSTs 
are asked to justify why an algorithm or strategy works, they often respond with a restatement of how to perform the algorithm (Ball, 1990a; J.-J. Lo et al., 2008). This indicates that PSTs may be unaware that there is a conceptual underpinning for the algorithm.

In their 2008 study, Lo, Grant, and Flowers provided insight into the learning challenges faced by PSTs as they attempted to make sense of multidigit multiplication in a course on number and operation. PSTs struggled to justify their strategies and to link strategies with visual models. Sometimes visual models were included without explanation and sometimes visual models were added "after the fact" and did not match the strategy actually employed. PSTs appear to hold a preference for the repeated addition interpretation for multiplication rather than the more powerful area interpretation (Graeber et al., 1989; J.-J. Lo et al., 2008). Researchers have found that the area interpretation of is more challenging for PSTs to understand, though it proves useful for some students in decomposing area into smaller regions (J.-J. Lo et al., 2008; Simon \& Blume, 1994). Baturo and Nason (1996) revealed a gap in PSTs' understanding of one and two dimensional units of measurement for multiplying in an area context. They indicate that the PSTs had "little or no understanding of how area measures evolve from linear measures when a formula for calculating an area is applied" (1996, p. 253), attributing PSTs' struggle to their prior experiences focused on procedural skills over conceptual understanding.

Prior research on PSTs mathematical knowledge has tended to focus on what they know and do not know, rather than how they learn (Mewborn, 2001; Thanheiser et al., 2014). Mewborn (2001) uses the term snapshot to refer to studies that either examine 
what a subject knows at a particular point in time or focus on pre and post data about student knowledge. A 2014 study by Thanheiser and colleagues indicated that out of 112 journal articles about PSTs' content knowledge, published between 1978 and 2012, 104 of the studies were snapshot or static studies of knowledge, while only 8 of the studies focused on motion studies of learning. In the content domain of whole number and operation, only 2 out of 26 studies were about PSTs learning, while the other 24 were static studies of knowledge. Mewborn (2001) and Thanheiser et al. (2014) advocate for more studies which focus on the development of PSTs' knowledge. This study addresses this gap in the research by presenting insight into PSTs' emerging models of meaning and strategy for multiplication.

\section{Realistic Mathematics Education (RME) design theory}

In traditional mathematics teaching, the instructor often begins with the result, such as a definition or algorithm, and then provides a series of examples of that result. Proponents of Realistic Mathematics Education (RME) argue that this traditional approach is backwards (Freudenthal, 1991; Gravemeijer \& Terwel, 2000). RME is an instructional design theory that focuses on beginning with contexts that are realistic or meaningful to students and building up ideas through a process of mathematizing (organizing mathematically), working from examples to more general conceptions.

Two of the primary design heuristics for RME are guided reinvention and emergent models. Within this section I briefly describe each of these ideas and highlight how they contributed to the design of the instructional sequence.

Guided reinvention. Guided reinvention is a design heuristic which focuses on creating contexts where students recreate important ideas in mathematics. According to 
Investigating Alternate Numeration Systems

Gravemeijer and Terwel (2000), "[t]he idea was to allow learners to come to regard the knowledge they acquire as their own, personal knowledge, knowledge for which they themselves are responsible" (p. 789). Generally, guided reinvention focuses on building up from student activity in contexts that are experientially real to the students. As students organize (mathematize) their ideas, they are able to build up new mathematical realities. This is also referred to as progressive mathematization, which can be broken down into two components: horizontal and vertical mathematization (Freudenthal, 1991; Gravemeijer \& Cobb, 2006). Horizontal mathematizing involves mathematizing reality. For example, if a student solves a base ten subtraction problem with manipulatives and then records that process with symbols, this activity is horizontal in nature because the student is organizing something that is real to the student. Vertical mathematizing relies on mathematizing prior mathematical activity. Returning to the prior example, if a student then uses this record process to create a general strategy for subtraction without needing to manipulate base ten pieces, this generalizing activity is vertical in nature.

The task sequence leveraged in the teaching experiment was based on an initial local instructional theory for supporting PSTs in reinventing an algorithm for multidigit multiplication. Because PSTs already have access to the formal mathematics of standardized algorithms in base ten (J.-J. Lo et al., 2008; Ma, 1999), I chose to shift from the routine context of base ten to the problematized mathematical context of base five (Chapter 6). The non-routine context of base five provides PSTs with an opportunity to set aside their known algorithm and experience the process of reinventing and refining an algorithm. 
Investigating Alternate Numeration Systems

Emergent models. As students engage in realistic mathematical contexts, their mathematizing activities can be described in terms of the emergent models they build. These emergent models are initially informal in nature and tied to the local context, but the models can become more formal as students engage in further exploration of similar contexts (Gravemeijer, 1998). In this paper I focus on an emergent model for whole number multiplication, describing this overarching model of multiplication in terms of two submodels: the emerging model of the meaning of multiplication and emerging model of strategies for performing multiplication. In the following section I provide background information on the underlying mathematics of multiplication. This formal background can be considered my own model for multiplication as the teacher/researcher.

Within RME, emergent models can be described using the models-of / modelsfor dichotomy, which captures different ways in which students use models (Gravemeijer \& Doorman, 1999; Johnson, 2013; Larsen, 2013). This language captures the idea of a shift from mathematizing in a local or situated context (creating a model-of the context) to vertical mathematizing as that model becomes a model-for more general or more advanced ideas. The emergent models heuristic is a tool for designing an instructional sequence, as well as a tool for describing how students' mathematical activity shifts as they engage with an instructional sequence. It also provides a framework for retrospectively analyzing students' mathematical activity (Johnson, 2013) and subsequently refining the mathematical tasks. I introduce the design heuristic of coemergence of models as a lens for both designing tasks and for interpreting students' mathematical activity which focuses on the interplay between related models. As a design heuristic, co-emergence of models is intended to expand on the heuristic of emergent 
Investigating Alternate Numeration Systems

models, providing a tool to leverage the interplay of multiple models or multiple aspects of an overarching model.

\section{The Mathematics of Base Five and Multiplication}

In this section I describe and define mathematical ideas and language which play a role in the remainder of the article, including an overview of the base five number system, and definitions of repeated addition, area, and partial products.

Explaining base five. The base five number system is built on powers of five, with place values corresponding to $5^{0}, 5^{1}, 5^{2}, 5^{3}$, etc. That is, in base five, there is a ones place, fives place, twenty-fives place, etc., rather than the ones place, tens place, hundreds place, etc. that appear in base ten. Consider the base five number $123_{\text {five }}$, which refers to 1 group of twenty-five, 2 groups of five and 3 ones (see Figure 5-1). To provide some language to talk about the numbers in base five, a group of five ones will be referred to as a long, a collection of twenty-five ones (or five longs) will be referred to as a flat, and a collection of one hundred and twenty-five ones (or five flats) will be referred to as a long flat (see Figure 5-1). Each time you collect five of one type of unit, those units can be regrouped into one of the next larger unit. For example, five longs can be regrouped as one flat. The numeral $123_{\text {five }}$ can be read aloud as 1 flat 2 longs 3 ones ${ }^{15}$. This base five language is taken from Bennett, Burton, and Nelson (2012a).

\footnotetext{
${ }^{15} 123_{\text {five }}$ should not be read aloud as "one hundred twenty three base five", as that confounds base ten and base five language. Base five does not have a hundreds place.
} 


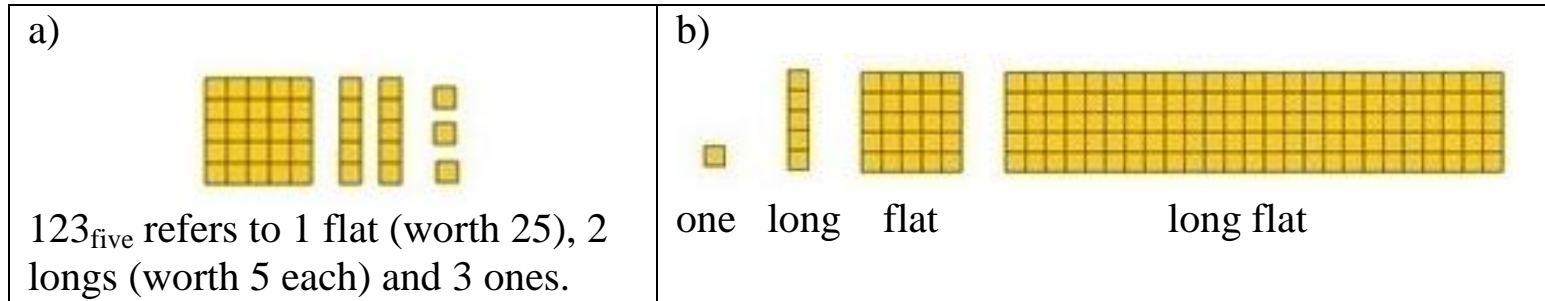

Figure 5-1. Base five language and description.

The purpose of using an alternate base within this study is to provide a novel context where PSTs do not have prior established algorithms for whole number operations. While base five is the alternate number system leveraged for this study, it would be relatively simple to modify the tasks for a different base number system, such as base eight. Base five was selected for this study because it was compatible with curriculum currently in use at the university where the study was conducted.

Defining the mathematics. In this section I describe key mathematical terms central to multidigit multiplication: repeated addition, area, and partial products. The mathematics described in this section can be interpreted as my own model for multiplication as the teacher/researcher, which I relate to the PSTs' emerging model for multiplication within the results section. Within this section examples are drawn from base ten. Multiplication of two whole numbers $\mathrm{A} \times \mathrm{B}$ can be defined as $\mathrm{A} \times \mathrm{B}=\mathrm{B}+\mathrm{B}+$ $\mathrm{B}+\ldots+\mathrm{B}$, where $\mathrm{B}$ is summed $\mathrm{A}$ times. The first number, $\mathrm{A}$, is the multiplier, which indicates the number of same-size groups being summed, and the second number, B, is the multiplicand, which indicates the size of each group. This definition of multiplication is referred to as repeated addition and serves as both an explanation of what multiplication means and a process for performing multiplication. While this is an appropriate definition for simpler problems such as $4 \times 6$, it does not scale well to solve larger problems, e.g. $123 \times 67$; fractional problems, e.g. $2 \frac{3}{4}$ × 12 1/2; or algebraic 
Investigating Alternate Numeration Systems

problems, e.g. $(x+2)(2 y+3)$. A visual reorganization of repeated addition into a rectangular array can be leveraged to create a more sophisticated interpretation for multiplication, linking repeated addition, arrays, and area as three interpretations of multiplication.

The area meaning for multiplication can be explained in terms of a rectangular image where the length and width of the rectangle correspond to the factors of the multiplication problem and the area corresponds to the product. The area interpretation of multiplication can be represented as a rectangular array where each unit in the product is represented with an individual cell/square; as a rectangle divided into rows, columns, or base ten shapes; or as an empty rectangle (Figure 5-2). Different versions of an area interpretation highlight different aspects of the meaning of multiplication. For example, the rectangles divided into rows or columns highlight the link between repeated addition and area.

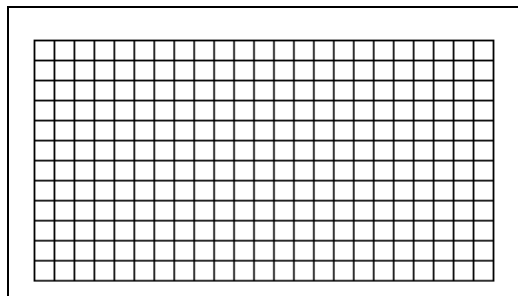

Area/array image, with an individual cell for each unit.

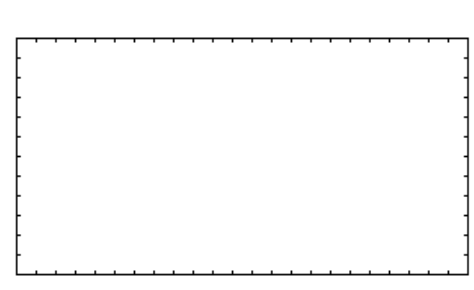

Area image as an empty 12 by 23 rectangle.

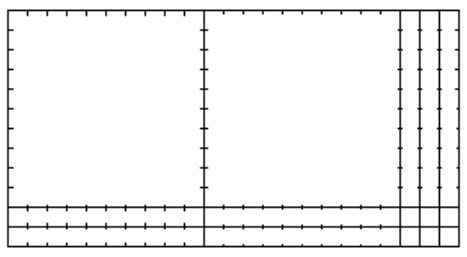

Area image subdivided to represent base ten pieces.

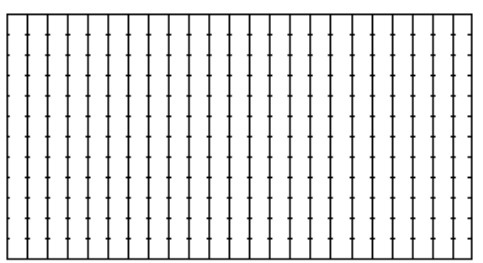

Area image presented as 23 columns of size 12 , promotes a connection between area and repeated addition $(12+12+\ldots+12)$

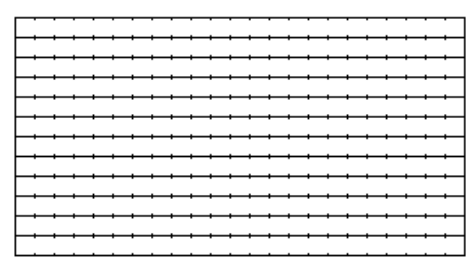

Area image presented as 12 rows of size 23 , promote a connection between area and repeated addition $(23+23+\ldots+23)$.

Figure 5-2. Five different area visualizations are provided for $12 \times 23$, highlighting different aspects of multiplication. 
The area interpretation of multiplication supports reasoning about multiplication in more sophisticated settings than repeated addition (such as larger numbers, fractions, and algebraic expressions). Area serves as a useful visual representation for dividing a complex multiplication problem into a collection of easier problems, using a strategy called partial products.

A partial product is the result when one part of a multiplier is multiplied by one part of a multiplicand. As can be seen in Figure 5-3, a partial products strategy involves (a) splitting a multiplication problem, such as $42 \times 31$, into sub-problems by splitting one or both factors into component pieces (often breaking each factor along place value lines, i.e. $42=40+2$ ), (b) multiplying sub-factors to find multiple partial products, and (c) summing the partial products to determine the total product.

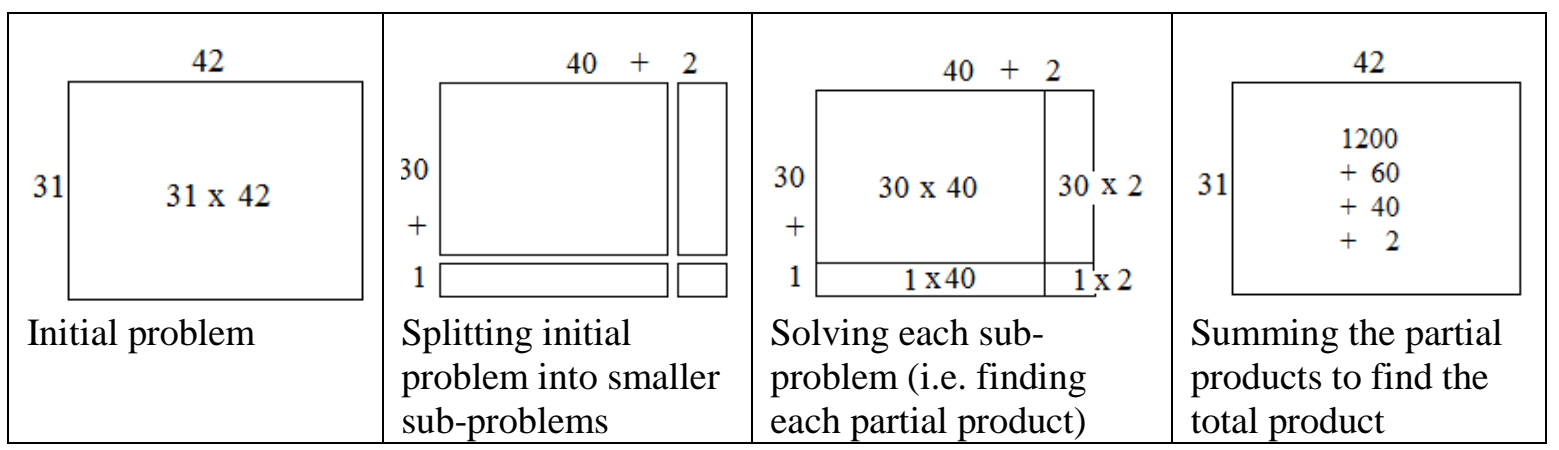

Figure 5-3. Partial products for $31 \times 42$

Partial products underlie the standard U.S. algorithm for multidigit multiplication.

As shown in Figure 5-4a, the problem $31 \times 42$ is written as two partial products ${ }^{16}(1 \times 42$ $=42$ and $30 \times 42=1260$ ) which are summed to find the total 1302. In elementary

\footnotetext{
${ }^{16}$ The standard algorithm generally involves computing four single digit calculations for $31 \times 42(1 \times 2,1 \times 4$, $3 \times 2,3 \times 4)$, however, the result is recorded in two rows as two partial products.
} 
curricula, students may also be introduced to an expanded version of the standard algorithm, in which $31 \times 42$ is written as four partial products, as seen in Figure 5-4b.

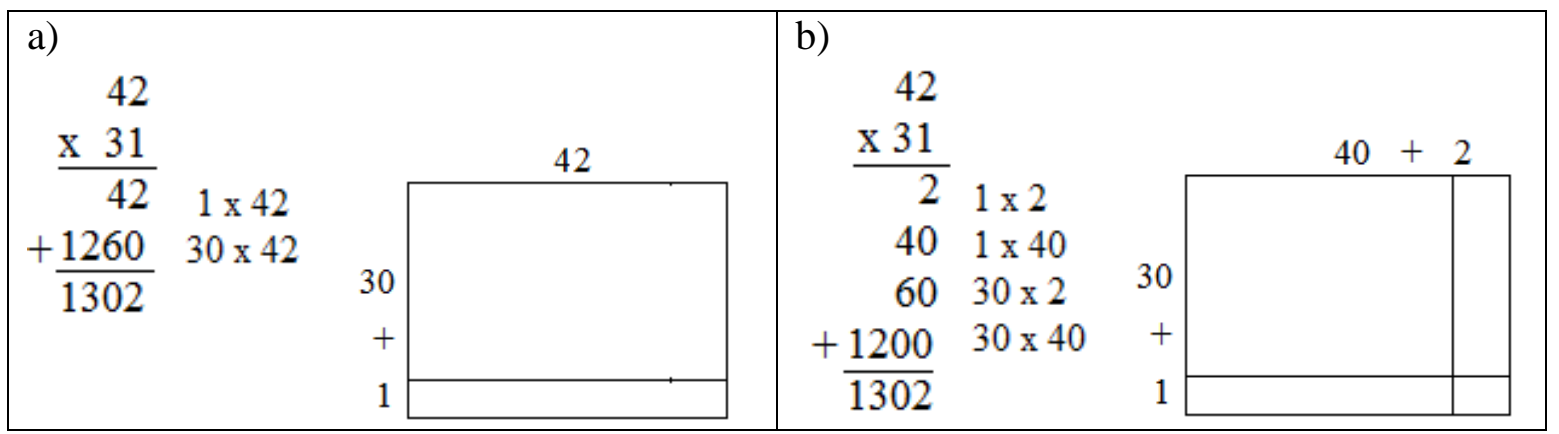

Figure 5-4. Connecting partial products strategies to standard algorithms for multiplication.

Next I provide a conceptual analysis of the multiplication, focusing on both the meaning of multiplication and strategies for performing multiplication.

Defining conceptual understanding. Hiebert and Lefvre (1986) describe two types of understanding, procedural and conceptual, which are commonly seen in the math classroom. Procedural understanding refers to knowledge of symbols, rules, and algorithms. Conceptual understanding is understanding where, "the linking relationships are as prominent as the pieces of information" (Hiebert \& Lefevre, 1986, pp. 3-4). PSTs tend to have procedural knowledge of elementary mathematics, but often lack conceptual knowledge, viewing mathematics as a disconnected set of rules (Ball, 1990a; Graeber, 1999; J.-J. Lo et al., 2008; Ma, 1999). In order to support the learning of their future students, PSTs must develop a conceptual understanding of the mathematics they will teach. A conceptual analysis of the meaning of multiplication includes (a) understanding that the number of square units within a rectangle corresponds to the product of the length and width, (b) connecting repeated addition to the area context, and (c) understanding and coordinating the one dimensional side lengths and two dimensional 
interior of the rectangle. A conceptual analysis of the partial products strategy for multiplication includes (a) being aware that a multiplication problem can be split into sub-problems, (b) purposefully and strategically splitting one or both factors in a multiplication problem, and (c) being able to connect partial products with the appropriate partial factors. For students using area imagery, this can be represented by connecting subsections of area to subsections of side lengths (see Figure 5-3). For students relying on a symbolic algorithm, these connections may be demonstrated by explicitly listing the sub-problems for each step (see Figure 5-4).

I do not intend for the above lists to be sequential. I anticipate that PSTs may develop the separate components in a variety of different and overlapping ways. The intention of providing a conceptual analysis for meaning and for strategy is to describe the richness and complexity of multiplication and to be clear about the mathematical model that I hold as the teacher/researcher.

The intentions of this report are (a) to introduce the design heuristic of coemergence of models, which is a refinement of the emergent models design heuristic, and (b) to contribute to the body of literature on PSTs learning about multiplication, leveraging the co-emergence heuristic as a lens to describe the interplay of two codeveloping mathematical models within student mathematical activity: a model for the meaning of multiplication and a model for how to perform multiplication.

\section{Methodology}

I conducted two teaching experiment cycles (Steffe \& Thompson, 2000), working with a pair of PSTs in each cycle as they engaged with the multiplication instructional sequence. The teaching experiments were designed to carefully analyze 
Investigating Alternate Numeration Systems

students' mathematical activity in order to identify principles (such as co-emergence) that could explain student activity within the instructional sequence and continue to elaborate/clarify the underlying local instructional theory. The intention of the teaching experiments was to focus on the collective mathematical activity of the PSTs, using the lens of the emergent perspective (Cobb \& Yackel, 1996) to focus on both individual and group mathematical activity in order to trace the development of key mathematical models. I served as both teacher and researcher during the teaching experiments.

Each cycle of the teaching experiment followed the "design, enactment, analysis, and redesign" model outlined by the Design-Based Research Collective (2003, p. 5). I conducted the first cycle during summer 2014 and the second cycle in fall 2014.

\section{Participants and Data Collection}

The participants in this study were preservice elementary teachers in the first term of a three term mathematics content sequence at a large urban university in the Pacific Northwest. The first course in the three-course sequence focuses on whole number and operation. The PSTs in the first cycle of the teaching experiment, Eli and Wendy, volunteered to participate in the task sequence as part of a four-week summer version of the course. The PSTs in the second cycle of the teaching experiment, Nora and Sarah, volunteered to participate in the task sequence in the two weeks prior to the start of the fall term as a head-start for the course on whole number and operation. All names used are pseudonyms. Brief descriptions of the recent math coursework for all participants are shown in Table 5-1. 
Table 5-1. Study Participants

\begin{tabular}{|ll|}
\hline Participants' Recent Mathematics Coursework \\
\hline Cycle 1 Participants & Cycle 2 Participants \\
Eli - Long break since prior math course & Nora - Freshman, had recently taken \\
(calculus) & algebra course \\
Wendy - Repeating the elementary & Sarah - Long break since previous math \\
content course after a prior low grade & course (prior math courses in the military) \\
\hline
\end{tabular}

All class sessions of the teaching experiment were video recorded and student work was collected and scanned. Approximately half of the teaching sessions were attended by a second researcher and the events of the sessions were discussed in debriefing meetings. After each teaching experiment, I wrote a reflection of what had transpired in the class sessions. Original video, transcriptions from the video, and my own notes all served as data sources, along with copies of student written work.

\section{Multiplication Task Sequence}

The creation of an initial local instructional theory (LIT) for reinventing a general strategy for multidigit multiplication began with a thought experiment about how PSTs could move from simpler to more complex multiplication problems and shift from situated to more general solution strategies. I leveraged the design heuristic of problematizing the mathematical context (Chapter 6) to shift from base ten to an alternate number system in order to place PSTs in a position where they do not have formal mathematics for performing multiplication. I initially conjectured that by sequencing multiplication tasks from single digit to two or more digits and including a specific focus on multiplication by the base $\left(10_{\text {five }}\right)$, PSTs would reinvent strategies for multiplication which leveraged splitting problems into partial products based along place value lines. I anticipated that a partial products strategy would initially emerge for specific problems 
and that this situated model of partial products could be generalized into an algorithm for multiplying any pair of base five numbers.

In pilot studies, I found that PSTs tended to rely on symbolic calculations when engaging with multidigit multiplication, performing operations on separate digits within a multiplication task without connecting to an appropriate definition of multiplication. When an area context was introduced within the pilot studies (either by the instructor or a PST), PSTs were able to leverage this meaning of multiplication to advance their multiplication strategies. This led me to revise the local instructional theory to support PSTs' emerging models for the meaning of multiplication alongside their emerging strategies for performing multiplication.

The first step of the LIT involves PSTs establishing multiplication as repeated addition, foregrounding this primary meaning of multiplication as a starting point. The second step of the LIT is developing a strategy for multiplication by the base $\left(10_{\text {five }}\right)$, a key step in creating multiplication strategies that leverage the structure of the place value number system. The third step of the LIT involves PSTs expanding their model for the meaning of multiplication to include area and array imagery. This third step supports the emergence of a more sophisticated model for the meaning of multiplication as larger problems are introduced and repeated addition becomes untenable. The fourth step of the LIT begins with subdividing the area representation into partial products and shifts to breaking numbers efficiently along place value lines. Within this fourth step, PSTs' model of partial products becomes a model for multiplying any pair of numbers, resulting in a general multiplication strategy. 


\section{Task 1: Repeated Addition}

a) Find the product $3_{\text {five }} \mathrm{x} 23_{\text {five }}$ and explain your solution strategy.

b) Create a definition of multiplication that would be appropriate to use with a $2^{\text {nd }}$ grader.

\section{Task 2: Times Base Rule}

Find the product $10_{\text {five }}$ x $23_{\text {five }}$ and explain your reasoning. Connect your explanation to a visual model.

Generalizations:

- Make a conjecture about multiplying by $10_{\text {five }}$. Justify your conjecture.

- Comparison across bases: $10 \times 23=230$ and $10_{\text {five }} \times 23_{\text {five }}=230_{\text {five. }}$. Is this a coincidence or something more?

\section{Task 3: Measuring a rectangle in base five}

(Each small group receives an unlabeled rectangle, size $42_{\text {five }} \mathrm{x}$ $31_{\text {five }}$ )

Your goal is to examine the size of the rectangle in base five. Determine the length, width, and area of the rectangle and discuss how the three quantities (length, width, and area) are related. Record two or more strategies for calculating the area and explain why those strategies make sense. Be sure to connect your visual models with written solutions.

Follow-up question: (Connecting repeated addition and area)

- In the first two base five problems, we used the repeated addition model for multiplication. How is your approach for Task 3 similar to the repeated addition model and how is it different than the repeated addition model?

\section{Task 4: Creating a general strategy}

Alice and Bob, who live in the land of Base Five, have a large box of rectangles. They need a strategy to find the area of any rectangle in base five. Your task is to create and explain an efficient strategy that will work to multiply any two base five numbers. Record your strategy on the provided paper.

Follow-up questions:

- Compare strategies with classmates

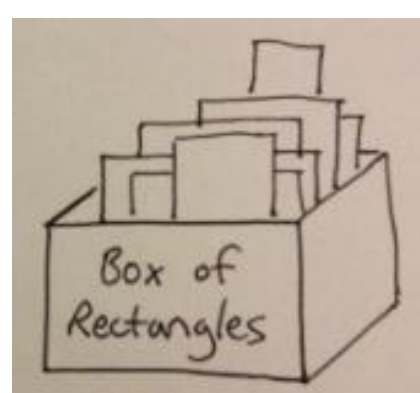

- Explain why the strategy makes sense

- Explain what is efficient about the strategy

- What is the role of the distributive property in each strategy?

- Describe a strategy for multiplying two base five numbers without having to draw a rectangle.

Figure 5-5. Multiplication Task Sequence

The multiplication task sequence for the teaching experiment was created using the initial LIT described above. The sequence of tasks (see Figure 5-5) is intended to 
promote student mathematical activity in a way that leads to the reinvention of a general strategy for multidigit multiplication.

Prior to the start of the multiplication task sequence, PSTs were asked to engage with a variety of tasks in alternate number systems, including the Roman, Mayan, Egyptian, and Babylonian historic number systems, as well as modern alternate base systems. Base five was used as the primary modern alternate base system. The task sequence described in Figure 5-5 captures the key elements of the tasks presented to the PSTs. While this provides an overview of the prompts given to PSTs, it is important to note that as teacher/researcher I added continuing presses for explanation and clarification in order to better understand the PSTs' mathematical activity. This included creating impromptu examples for the PSTs to explore and asking PSTs to elaborate on and compare representations and strategies.

After each session of the teaching experiment, I reflected on the PSTs' work and refined my plans for the next session. This between session analysis was vital to making sense of PSTs' mathematical activity and to preparing for subsequent sessions. However, the bulk of the analysis occurred after each full cycle. In the next section I outline the steps of my analysis process.

\section{Analysis Process}

A retrospective analysis provides an opportunity for a thorough historical analysis of what occurred during a teaching experiment, particularly across multiple cycles. Having an idea of what mathematical models emerged, I was able to attend to what PST mathematical activity led to those models, and analyze how and why the instructional sequence supported PSTs' emerging models (Steffe \& Thompson, 2000). 
Investigating Alternate Numeration Systems

The intention of the analysis was to refine the initial LIT, establishing design principles that explain how students' reinvention activity can be supported. The retrospective analysis of the teaching experiment is an iterative process, which I have separated into four major phases, with the first two phases repeated after each cycle of the teaching experiment and the latter two phases analyzing across the cycles (Figure 5-6). The analysis is based on the cyclic process described by Lesh and Lehrer (2000) and Cobb and Whitenack (1996), and modeled after a cyclic design experiment conducted by Larsen (2004).

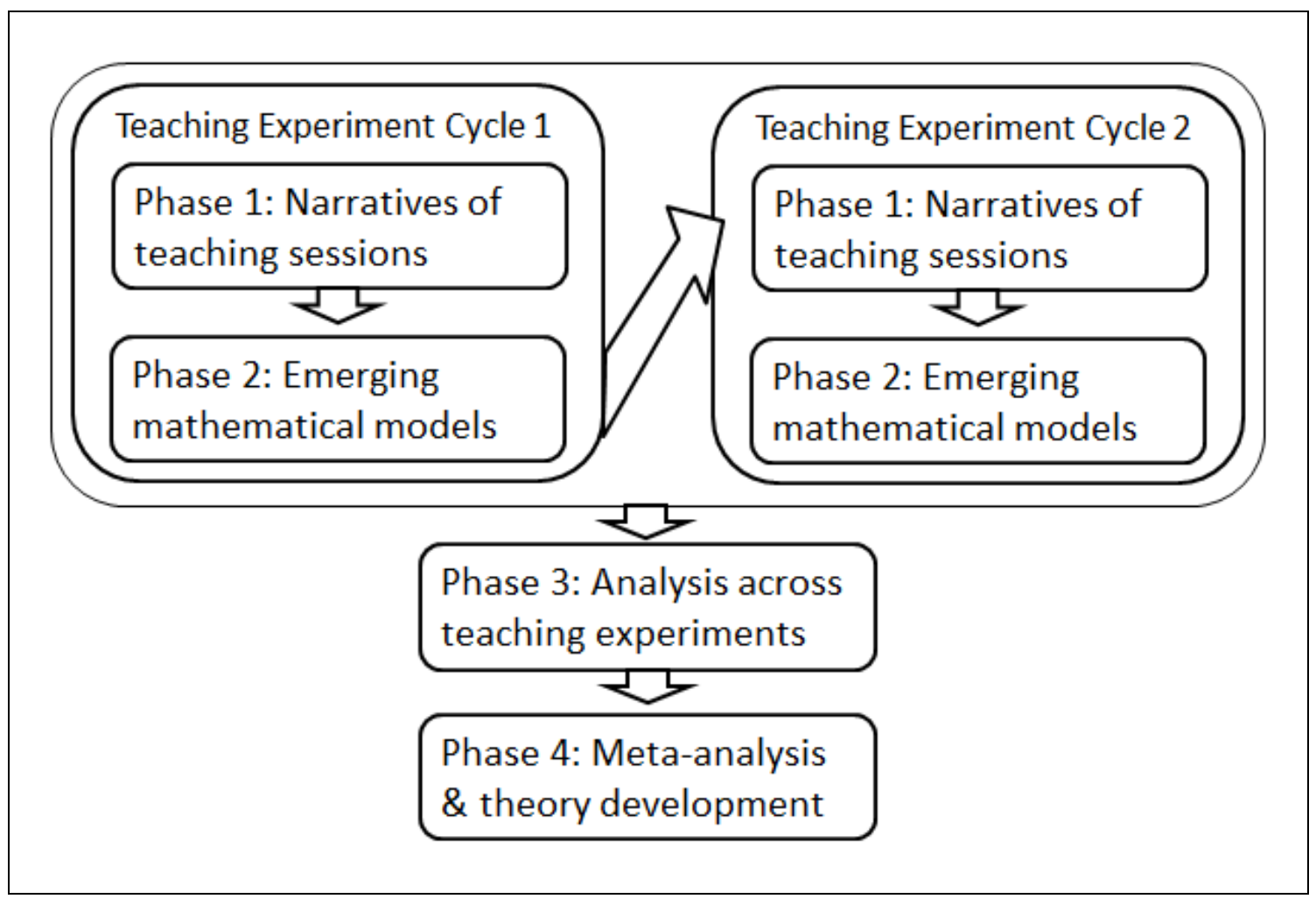

Figure 5-6. Phases in analysis

Phase 1: Creating narratives of the teaching sessions. In phase one, I worked chronologically, creating a content log of the videos for each teaching session. In order to manage the data, I chunked it into coherent episodes. For this study, an episode refers 
Investigating Alternate Numeration Systems

to a collection of dialogue and student work that corresponds to one solution attempt or one line of reasoning. Episodes often centered around moments of struggle, new strategies and new definitions, discussions of procedures, comparisons across bases, generalizations, and justifications.

An example of a brief episode is shown in Figure 5-7, where Eli and Wendy are responding to a prompt to create a definition for multiplication. This PST generated definition captures their understanding of multiplication as repeated addition, making sense of the first number as the multiplier and the second as the size of the multiplicand. Each session contained many episodes, resulting in far more data than could be included in this report. I selected episodes for this report in order to (a) highlight the overall trajectory of PSTs' mathematical activity and (b) focus attention on the explanatory power of co-emergence to describe the interplay of developments in the meaning of multiplication and developments in strategy within the PSTs' emerging multiplication model.

Eli: We could say, just say that the first number is the number of groups, and the next number is the number of things

Wendy: In that group

Eli: In those groups, yeah, Wendy: okay

Figure 5-7. Example of an episode where preservice teachers are asked to define multiplication in language appropriate for children.

I created a narrative of each session. This narrative included descriptions of episodes and transcriptions of selected portions of the session, as well as images of student work. The narrative was annotated with conjectures for explanations of student mathematical activity. The goal of phase 1 was to become familiar with the data and to begin to organize the data. 
Investigating Alternate Numeration Systems

Phase 2: Emerging Mathematical Models. For each cycle of the teaching experiment, I built detailed reports of the PSTs' emerging models for the meaning of multiplication and their emerging models for strategies. The reports included descriptions of student mathematical activity supported by transcript, photographic evidence, and student written work. I used the design heuristic of emergent models as a lens to describe shifts in the student mathematical activity.

In phase 2, I included an explicit focus on aspects of the tasks, including student dialogue and questions, which impacted the development and shifted the use of the models. Existing research and domain specific frameworks, such as Graeber, Tirosh, and Glover's (1989) work with PSTs' primitive models for operations and Baturo and Nason's (1996) research on PSTs' understanding of area measurement were used to provide rationales and insights for students' mathematical activities.

While phases 1 and 2 were done separately for each of the 2 cycles of the teaching experiment, phase 3 focused on analyzing across both cycles.

Phase 3: Analyze Across Teaching Experiments. The goal of phase 3 was to analyze student mathematical activity for particular tasks across the two teaching experiment. For example, I examined how the PSTs worked with Task $2\left(10_{\text {five }}\right.$ x $\left.23_{\text {five }}\right)$ to explore (1) how students made sense of multiplying by the base ( $\left.10_{\text {five }}\right),(2)$ what sorts of generalizations they were able to make, and (3) what appeared to prompt those generalizations. For example, one local generalization was that multiplying by $100_{\text {five }}$ causes the digits of a number to shift two places to the left, while a more global generalization is that multiplying by the base in any alternate base system causes the 
digits of a number to shift to the left one place. The reports generated in phase 2 played a central role in the analysis for phase 3.

Phase 4: Meta-analysis and Theory Development. Similar to the final phase of analysis described by Cobb and Whitenack (1996), in this phase I analyzed the prior analyses with the intention of creating a chronology of the emergence of models across the two cycles of the teaching experiment. This chronology drew from both cycles and served as the basis to consider modifications to the instructional sequence and anticipated student mathematical activity. It was during this phase of meta-analysis that I stepped past leveraging the tools of RME and considered ways to advance RME theory. I considered the complex situation of how to make sense of student mathematical activity as students developed two distinct, but related models for multiplication. How could I capture the interaction of the two emerging models? This co-development, which I refer to as co-emergence of models, is a focus of the following results section and serves as a lens or tool to organize students' mathematical activity throughout the task sequence (see Figure 5-8), as well as a heuristic for supporting student's mathematical activity.

\section{Results and Discussion}

In this section I focus on the PSTs' mathematical activity as they build a general strategy for multidigit multiplication in base five. I argue that the PSTs' model for the meaning of multiplication co-developed alongside their model of a strategy for performing multidigit multiplication. I introduce the term co-emergence of models to refer to the phenomenon of having multiple models emerge together in such a way that each model aids in the development of the other model. Co-emergence focuses attention on the complex process of leveraging one partially constructed model to help build up 
another model and vice versa. The theory of co-emergence is intended to advance the theory of emergent models by addressing the interplay between related emerging models. It is possible to consider student mathematical activity in terms of a single complex model, with meaning and strategy as aspects of that model. (In this case, co-emergence of models could be rephrased as co-emergence of aspects of a model.) However, I have chosen to treat the emergence of a model for multiplication as the emergence of two related models, leveraging the explanatory power of splitting a complex idea into simpler pieces.

The results section is split into a series of episodes. The first two episodes correspond to the first two tasks in the task sequence, focusing on repeated addition and grouping by the base $\left(10_{\text {five }}\right)$. The next two episodes correspond to the third task in the task sequence as PSTs' mathematical activity includes both repeated addition and area representations for the meaning of multiplication and the PSTs begin to articulate a partial products strategy. The final episodes focus on PSTs developing a general strategy for multidigit multiplication relying on partial products (Task 4). Within each episode I discuss the utility of co-emergence as a lens for student mathematical activity. .

A guiding visualization for the co-emergence of the two models is provided in Figure 5-8. The left column represents steps in the emerging model of meaning for multiplication and the right column represents steps in the emerging strategies for multiplication. The downward arrows on the left and right of the image represent the emergence of each separate model. The co-emergence of the models is captured by the central arrows, each labeled with the episode which highlights the interplay between the two models. 


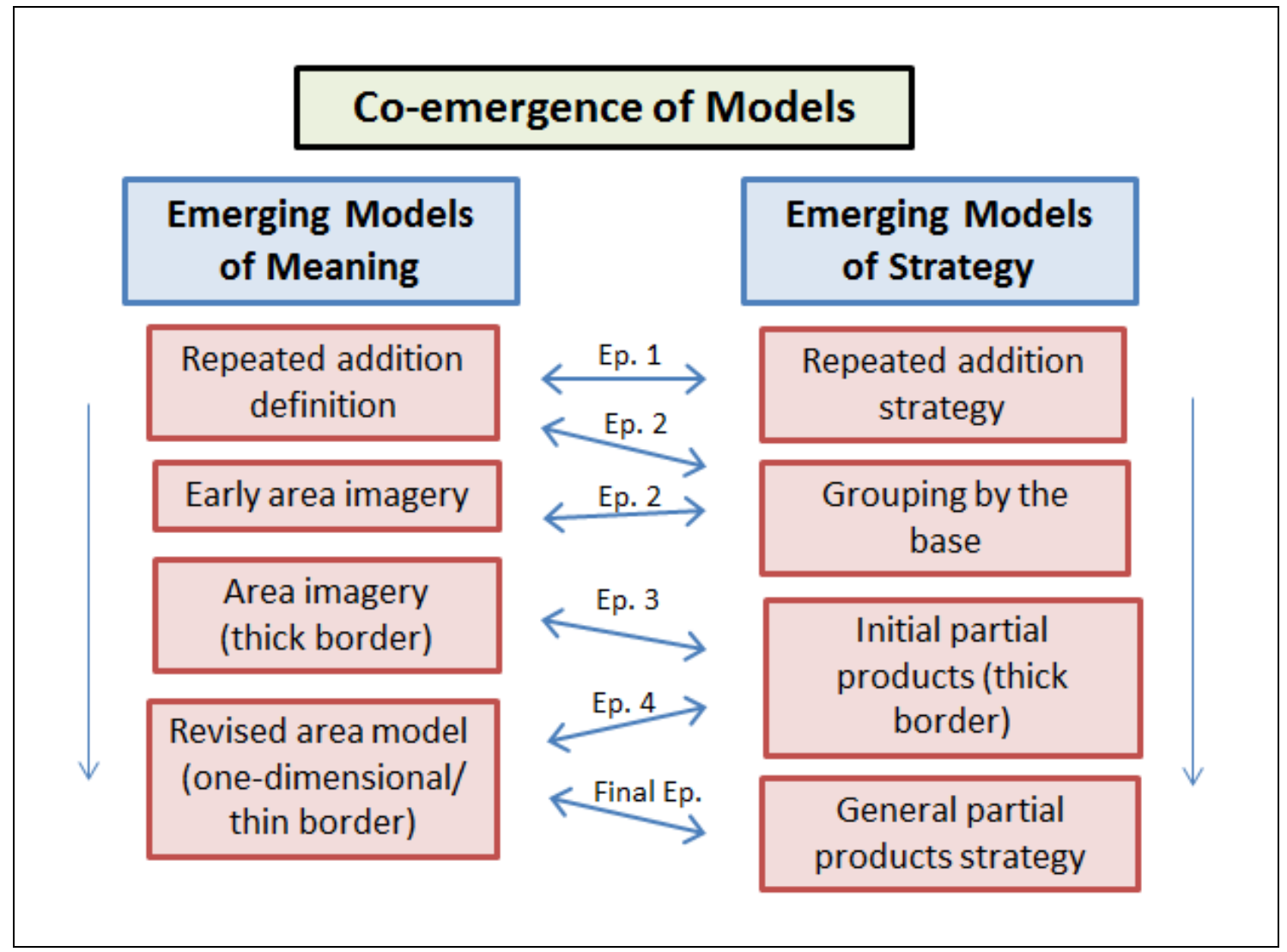

Figure 5-8. The co-emergence of models of meaning and models of strategy highlights the interplay between models.

\section{Episode 1: Repeated Addition Co-emerging as a Definition and a Strategy}

The first episode focuses of the initial starting point for both meaning and strategy for multiplication and the relationship between the two. The multiplication sequence began with a one digit by two digit task (Task 1, Figure 5-5). The small multiplier of 3 was purposely selected in order to promote repeated addition as a reasonable strategy for solving the multiplication task.

After a brief negotiation about remaining in base five rather than translating to base ten, Eli and Wendy began the problem by recognizing that $3_{\text {five }} \times 23_{\text {five }}$ referred to 3 copies of $23_{\text {five. }}$ 
Wendy: Ah, I could, OK, you do it the little kid way. Just saying that you have three of this (picking out the correct manipulatives to make three groups of $23_{\text {five }}$ ).

Eli: $\quad$ That's what I was just thinking too.

Wendy: The little kid way. It's kind of the little kid way. Well I guess is the simplest way I should say. So it's three groups of this... just add them up from here.

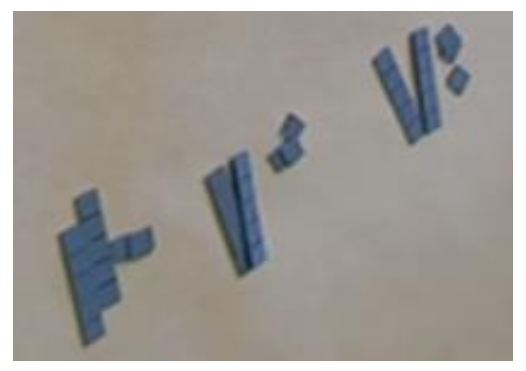

Figure 5-9. Wendy and Eli built 3 groups of manipulatives, with 2 longs 3 ones per group.

Researchers refer to the repeated addition model of multiplication as the primitive model, indicating that it is the primary model of multiplication for both children and PSTs (Fischbein et al., 1985; Graeber et al., 1989). Wendy recognized the primitive or intuitive nature of the model as she associated it with how a "little kid" would perform multiplication.

The repeated addition model served as a definition of multiplication, helping the PSTs to unpack the symbolic problem $3_{\text {five }} \times 23_{\text {five }}$ into one which was represented as 3 collections of 2 longs and 3 ones. Once visualized as 3 collections of $23_{\text {five, }}$ the PSTs were then able to rearrange and regroup the quantities to find a product of $124_{\text {five. }}$. When asked to create a definition for multiplication, they were able to explicate the "little kid" method in clearer language.

Eli: We could say, just say that the first number is the number of groups, and the next number is the number of things

Wendy: In that group

Eli: In those groups, yeah, Wendy: okay 
Investigating Alternate Numeration Systems

At this point, the PSTs held a definition of multiplication as repeated addition. In this definition, the PSTs described that the first number indicated the number of groups (multiplier) and the second number indicated the size of the groups (multiplicand). Repeated addition can be leveraged to describe the PSTs' emerging models in two distinct ways. The PSTs' emerging model for the meaning of multiplication, as seen in the PSTs' definition, aligns with the definition of multiplication as repeated addition. The PSTs' emerging model for performing multiplication, creating three collections of size $23_{\text {five }}$, is a repeated addition strategy. At this point, it may be difficult to separate meaning from strategy in the co-emerging models, but as the problems became more complex, the differences between meaning and strategy became more apparent.

\section{Episode 2: Leveraging Repeated Addition to Make Sense of Grouping by the Base}

The primary purpose of the second episode is to demonstrate how the PSTs' initial model for the meaning of multiplication (repeated addition) supported a key development in a strategy for performing multiplication (grouping by the base). A secondary purpose of this episode was to highlight how creating a justification for a strategy of grouping by the base led one set of PSTs to begin to use area imagery for the meaning of multiplication. Both of these purposes are indicated with arrows in Figure $5-8$.

Task 2 was designed to focus PSTs' attention on multiplication by the base $\left(10_{\text {five }}\right)$. Multiplication by $10_{\text {five }}$ in base five plays the same role as multiplication by $10 \mathrm{in}$ base ten. Gathering $10_{\text {five }}$ copies of a given unit allows one to exchange for the next larger unit. Fasteen, et al. (2015) provide more details on PST mathematical activity 
associated with this particular task. In order to approach the task of multiplying $10_{\text {five }}$ by $23_{\text {five }}$, Wendy and Eli interpreted the problem as the repeated addition of $23_{\text {five }}$ five times (Figure 5-10). Their model for the meaning of multiplication as repeated addition supports their approach to the initial multiplication task, as well as their investigation of a generalization for multiplying any number by $10_{\text {five }}$.

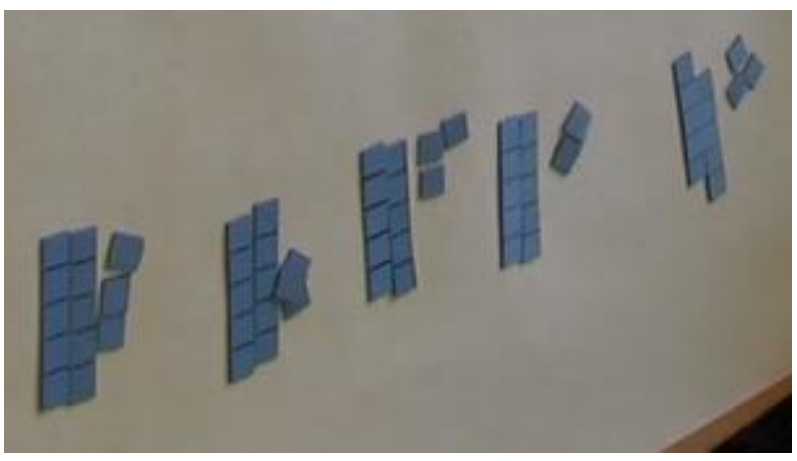

Figure 5-10. Wendy represented $10_{\text {five }} \mathrm{x} 23_{\text {five }}$ by creating five piles of $23_{\text {five. }}$.

The PSTs in both cycles are able to generalize the problem $10_{\text {five }} \times 23_{\text {five }}$ to multiplying any base five number by $10_{\text {five }}$, recognizing that this would cause all of the digits in the number to shift to the left. When asked to justify why this rule would work in general, the PSTs focus on why each base five piece would shift when multiplied by $10_{\text {five. }}$. Figure 5-11 provides examples of Nora and Sarah's work with justifying why a long times a long is a flat (i.e. $10_{\text {five }} \mathrm{x} 10_{\text {five }}=100_{\text {five }}$ ). While PSTs in both cycles used repeated addition to argue for why a long times a long is flat, I chose to draw data from cycle 2 for this episode because Nora and Sarah's argument also led to the emergence of an area image.

Parts (a) and (b) of Figure 5-11 demonstrate how repeated addition, as both a meaning and a strategy, supports the justification for why multiplying by $10_{\text {five }}$ causes each unit to shift to the next larger unit. Part (a) demonstrated multiplying a long by one, 
then two, etc., until one reached five copies of a long, which was then regrouped as one flat. The written explanation in part (b) used repeated addition language to unpack the meaning of multiplying a long times a long.

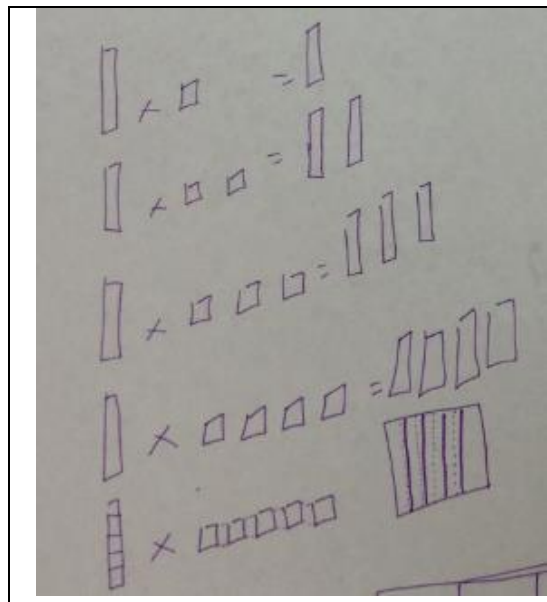

a) Leading up to 5 copies of a long, or long $\mathrm{x}$ long $=$ flat.

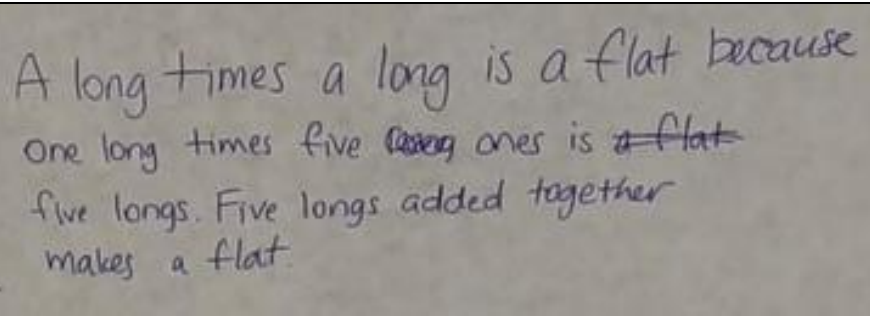

b) Students justified a special case of the Times Base Rule, namely, long $\mathrm{x}$ long = flat.

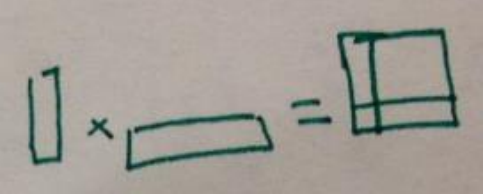

c) One long represented height, the other width, to create a flat.

Figure 5-11. Sarah and Nora recorded strategies for making sense of why long $\mathrm{x}$ long = flat. These strategies leveraged both repeated addition and area interpretations for the meaning of multiplication.

When asked to justify why a long times a long would be a flat, Sarah creates an image of a flat that shows one long as the width and the other long as the height (Figure 5-11, part (c)). Sarah connected rectangular imagery and multiplication, stating "I like the idea of the second one gets put on its side. And the first one, you have to stack them up that tall.” Her language, together with her drawing, indicated that she was considering the first number as the height and the second number as the width, signaling the early emergence of an area or array interpretation of the meaning of multiplication, rather than repeated addition.

Engaging in the process of creating a justification for why multiplying by $10_{\text {five }}$ causes digits to shift a place value to the left provided an opportunity for Sarah and Nora 
to begin to reorganize their underlying meaning of multiplication to include language related to area. In this way, we witness the co-emergence of a new strategy (involving grouping by the base) alongside the beginning of a more sophisticated meaning for multiplication (involving area). Both of these co-emerging models (for meaning and strategy) undergo additional advancement in the following episodes.

\section{Episode 3: Co-emergence of Thick Border and Initial Partial Products}

In the third episode I argue that PSTs' initial interaction with an area context problem revealed that (a) their understanding of the meaning of multiplication as area was not in alignment with the standard way of representing area with one dimensional borders and two dimensional interiors and yet (b) this emerging model for the meaning of multiplication was sufficient to support an initial partial products strategy. The essence of co-emergence is the back and forth nature of the emergence of models, allowing the student (and teacher/researcher) to leverage one partially formed model to support developments in the other.

In task 3 (see Figure 5-5), the PSTs were presented with a rectangular garden (dimensions $42_{\text {five }} \times 31_{\text {five }}$ ) and asked to find the length, width, and area of the rectangle in base five. They used the base five manipulatives as measuring tools to find the length and width. Eli and Wendy recognized a connection between area and multiplication, indicating that the answer to the multiplication problem $420_{\text {five }} \times 310_{\text {five }}$ was the number of squares in the rectangle. After Eli stated that the formula for finding area was length times width, he was prompted to explain why. Eli said:

You are taking, you're trying to find how many. It's a very basic question I haven't actually thought about in a long time. You are trying to find how many squares are in it [the rectangle]. Those are going to come up with whatever this is 
Investigating Alternate Numeration Systems

(pointing to where the answer would go in the multiplication problem $420_{\text {five }} \mathrm{X}$ $310_{\text {five }}$. So if you think about it as, there's 3 flats 1 long rows here... And there's 4 flat 2 long rows going this way. Then how many one squares does that make up? Right?

The rectangular area context resonated with the PSTs as a context for multiplication. This could be seen in Eli's claim that the number of squares in the rectangle was the answer to the multiplication problem $420_{\text {five }} \times 310_{\text {five. }}$. He attempted to connect the two factors to the number of rows and columns of squares within the rectangle, indicating an underlying array interpretation of multiplication. PSTs in both cycles treated area as a reasonable context for multiplication.

While Eli verbalized a connection between area and multiplication, he and Wendy struggled to correctly identify the side lengths of the rectangle. In both cycles of the teaching experiment, PSTs initially created a border along the edge of the rectangle using manipulatives that were five units thick (see Figure 5-12). In both cases, this resulted in the PSTs stating that the rectangle was $420_{\text {five }} \mathrm{x} 310_{\text {five }}$, rather than the correct $42_{\text {five }} \mathrm{x}$ $31_{\text {five }}$, using the area of the thick border in place of the lengths of the rectangle. This error indicated that although PSTs recognized a connection between the area context and multiplication, at this point in the instructional sequence their understanding of the area interpretation of multiplication did not align with standard interpretation of area with one dimensional borders and two dimensional interior. This error may have been related to the two dimensional manipulatives that were leveraged as measuring tools. Even Sarah and Nora, who were able to draw an appropriate image of a long times a long in Figure 5-11 (part c), were not able to correctly identify the side lengths of the given rectangle. Despite these flaws in PSTs' emerging model of the meaning of multiplication, once the 
area context was introduced, the PSTs in both cycles discontinued use of the basic repeated addition strategy. Instead, they relied on the area imagery to support their multiplication activities.

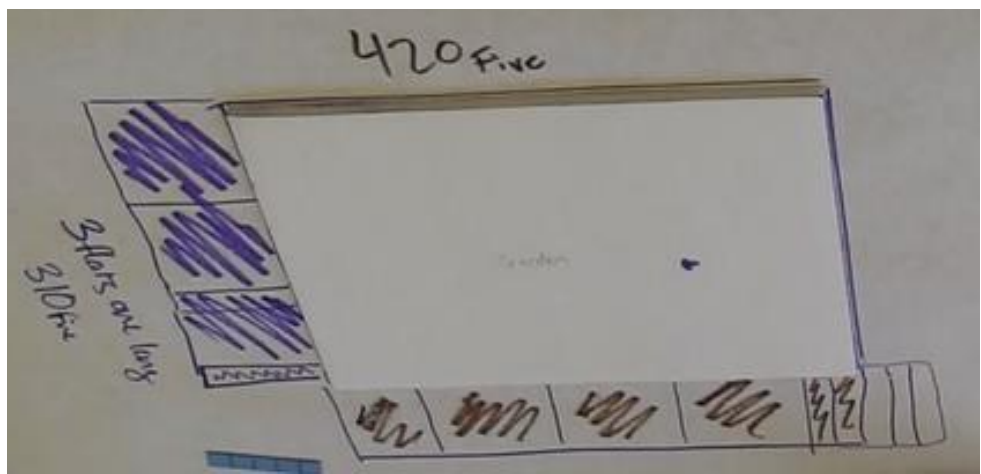

Figure 5-12. Wendy and Eli measured the rectangle in base five, creating an overly thick border.

Having created an overly thick border for the $42_{\text {five }} \mathrm{x} 31_{\text {five }}$ rectangle, the PSTs then began to calculate the interior area. Initially I hypothesized that the PSTs would self-correct their thick border error when they began filling the rectangular image with base five manipulatives. Because the shape could be physically filled with flats, longs, and ones, I hypothesized that filling the rectangle with the correct area pieces would cause the PSTs to question their overly thick border. However, instead of relying on the actual physical size of each subsection of area to determine its value, the PSTs chose to calculate the area of the subsections by multiplying portions of the thick border. For example, in Figure 5-13, Eli and Wendy began by outlining a subsection of area in the upper left corner. 


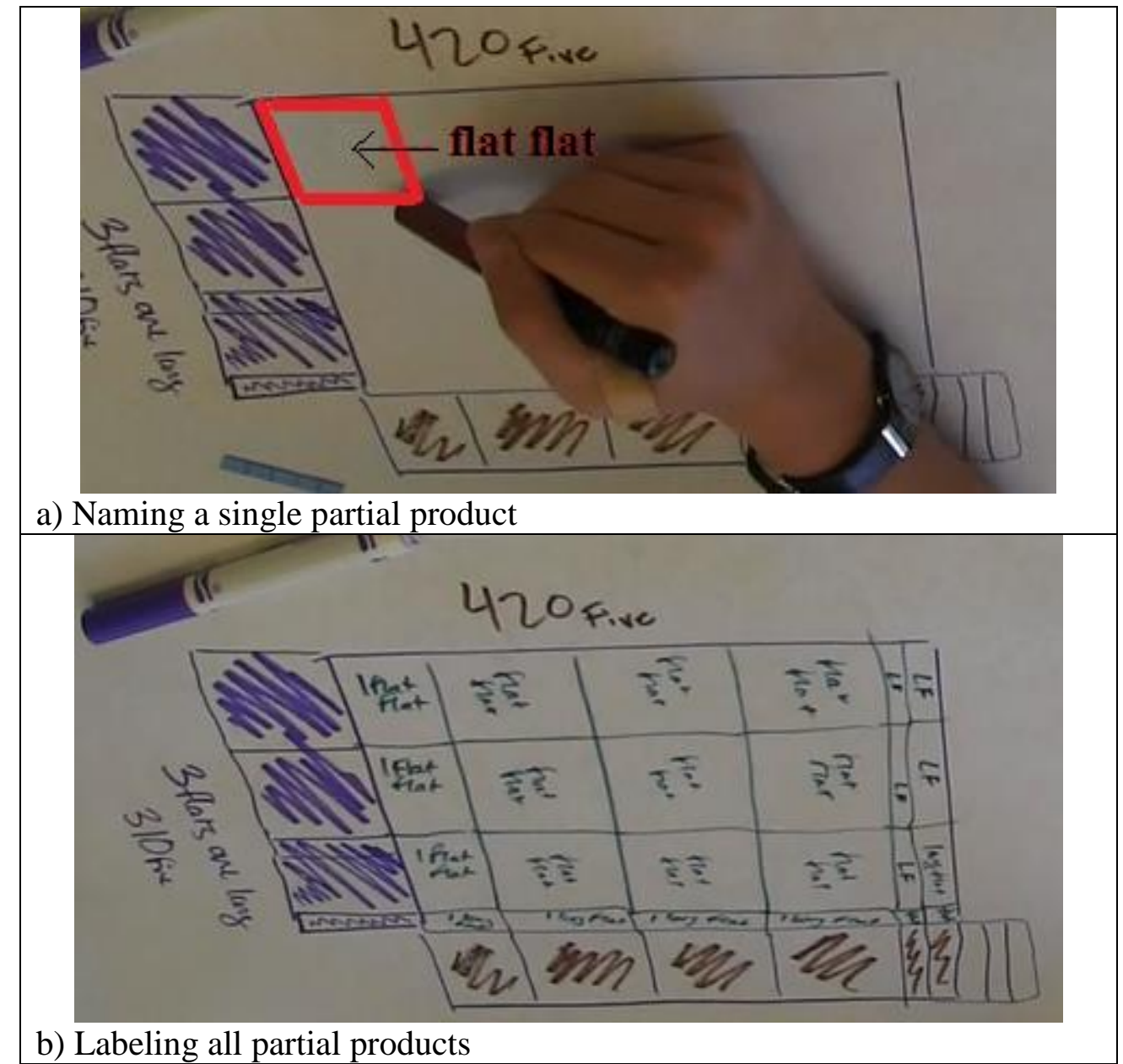

Figure 5-13. Eli and Wendy determined the area of the rectangle, finding a value 25 times larger than appropriate.

Although the shape they drew is the size of a flat, they determined its value by multiplying the two corresponding subsections of the thick border (both were flats). This resulted in a partial product worth 25 times more than a flat, which is referred to as a flat flat in base five (worth 625 in base ten). Figure 5-13 (part b) shows the rectangle split into subsections based on this partial products strategy.

One advantage of a co-emergence lens is that it allows the teacher/researcher to parse the co-developing models and identify an error in one model while recognizing an advancement in the other model. In this episode, the PSTs' area interpretation for the 
meaning of multiplication contains a fundamental flaw with the thick border. However, this flaw does not prevent a key advancement in strategy, where a complex problem (finding the total area) is split into a collection of simpler problems (partial products.) Ironically, the flaw in the PSTs' interpretation of area provided an unexpected advantage to me as the teacher/researcher, revealing evidence of how the PSTs had determined the area. The PSTs' strategy involved splitting the larger rectangle into many smaller components, with Figure 5-13b containing 24 sections within the rectangle. In order to find the value of each section, the PSTs looked to the corresponding side lengths of the section. If the PSTs had determined the correct linear border of long by long for Figure 5-13a, then the partial product of one flat could have been the result of either calculating a partial product or a simple geometric filling of the corner. However, the error of flat flat indicates that the value of the corner, as well as the rest of the sections in the rectangle, was determined by calculating partial products rather than geometric filling.

In the next episode, I highlight how the initial partial products strategy was leveraged to revisit and improve upon the earlier (incorrect) area model.

\section{Episode 4: Leveraging a Single Partial Product}

In order to address the error in the area imagery in Episode 3, I first attempted to confront the error head-on. Referring to the rectangular garden image in Figure 5-13 (part b), I asked Wendy and Eli "How big is a flat flat? Use hand gestures here. How big is a flat flat?" Gesturing with manipulatives, they indicated that the shape would be a 25 by 25 square, larger than the rectangular garden they were working with. Seeing that 
Investigating Alternate Numeration Systems

be the size of the dozen of these?" Eli responded, “No, it doesn't look like it, does it? What are we doing wrong?" At this point, both Eli and Wendy became aware of their error, but appeared stuck in what to do next.

As the teacher/researcher, I made an in-the-moment choice to leverage the PSTs' initial use of a partial products strategy to support their co-emerging model of the meaning of multiplication as area. The partial products strategy involves splitting a large problem into smaller, simpler problems. I chose to focus on their first partial product (flat $\mathrm{x}$ flat $=$ flat flat) and to create a prompt that would ask them to examine the problem in reverse, first determining the area of a flat and then finding its length and width.

Holding up a single flat, I asked "This guy, what's its area?"

Eli: $\quad$ Its area would be 25 .

I: What would it be in base five?

Eli: $\quad$ It would be -

Wendy: - one flat

I: What would its length and width be?

Eli: $\quad$ One long by one long

I: $\quad$ Okay. How many, if you had to guess, would fit in your garden?

Eli: Okay. It would probably be, well because we've drawn them all out already, it would be 12 .

The PSTs were able to focus on a single flat and determine first the area, and then the length and width of one flat in base five. Because this partial product corresponded to a single flat, which was already part of the PSTs' mathematical repertoire (as seen in Episode 2), the PSTs were able to make sense of the length, width, and area of the shape. When asked how many of this shape would fit in their garden, Eli recognized that their rectangle has already been split to show 12 flats (the shapes labeled as flat flat in Figure $5-13)$. 
Having recognized a flaw in their earlier method for finding the area of the garden, Wendy and Eli returned to the $42_{\text {five }} \times 31_{\text {five }}$ rectangle to unpack their prior error.

Wendy: I think, you know what I think we did wrong?

Eli: What did we do wrong?

Wendy: We made it too big, because we're multiplying, we did it - Oh I figured it out, we did it a step up. ... Instead of doing one flat here [K gestures to a flat within the thick border], we should have done a long by a long. And multiplied it by a long by a long. And that makes it -

Eli: - Because we're including all of this [pointing to the thick border] in our multiplication when we should just be doing, it should be in one's, basically. So that's one long, two long, three long, four long two [E indicates that the side should be measured as 4 longs 2 ones]. So it's not this [ $420 \times 310]$. It's that [he writes $42 \times 31]$.

Wendy suggested that the side lengths were measured with the wrong type of objects. When she said they "did it a step up" she was referring to using flats rather than longs to measure the lengths. Eli recognized that this has caused them to determine a side length that was much larger than would make sense, thus leading to a much larger product than would be appropriate.

In the second cycle of the teaching experiment, Sarah worked with the same error of multiplying a two dimensional flat by a two dimensional flat. In unpacking her error, Sarah determined that "that would be area of an area, so that actually makes it an oddly intersecting four-dimensional-- You now have a garden in hyperspace.” She jokingly referred to the result as a "hypercube," indicating that it would be a four dimensional shape and not at all appropriate for a flat rectangular garden. By recognizing that their error was a result of multiplying a two dimensional object by a two dimensional object to create something much too large, the PSTs became aware of the importance of coordinating two different types of units in within area: one-dimensional linear units and 
two-dimensional area units. This realization had an immediate impact on the PSTs emerging model for the meaning of multiplication as area.

Having made sense of the single partial product of 1 long $\mathrm{x} 1$ long = 1 flat, the PSTs in both cycles were primed to then modify the rest of their $42_{\text {five }} \times 31_{\text {five }}$ rectangle. They were able to use appropriate linear units to find the side lengths and then determine the correct area within the rectangle. This modification (see Figure 5-14) cascaded through the other partial products to result in a key shift in the model of the meaning of multiplication as area. Namely, the PSTs recognized that the factors of a multiplication problem correspond to linear units (one-dimensional) while the product corresponds to area units (two-dimensional). In other words, they recognized the need to measure the side lengths of the rectangle with linear units and the area with square units. Given that prior research indicates that PSTs' understanding of multiplication and dimension tends to be "incorrect, and/or incomplete, and often unconnected" (Baturo \& Nason, 1996, p. 261), this explicit focus on coordinating dimensions appears promising.

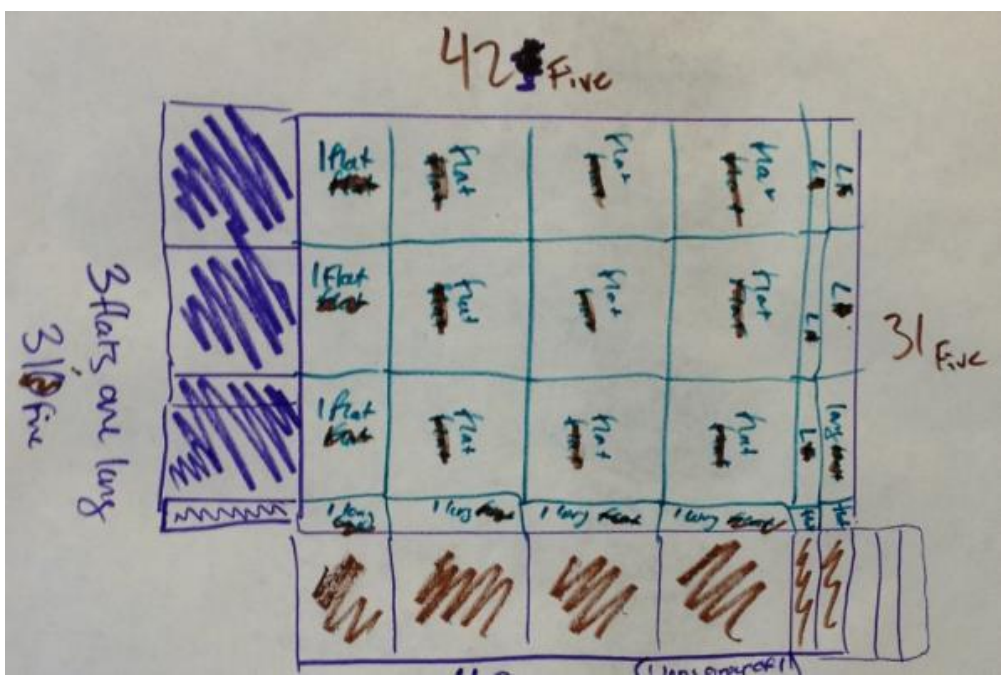

Figure 5-14. Wendy and Eli modified their prior image of $420_{\text {five }} \times 310_{\text {five }}$ to match $42_{\text {five }}$ x $31_{\text {five }}$, scribbling over the word "flat" to change the unit type for each subsection. 
Investigating Alternate Numeration Systems

Prepared with an emergent model of the meaning of multiplication as area, the PSTs were appropriately situated to engage with the task of creating a general strategy for multiplying multidigit numbers. In this context, area shifted from its role as a context for students' mathematical activity in making sense of the meaning of multiplication and it became a representation for supporting the process of creating general strategies for performing multiplication. The shift from making sense of the area context to leveraging the area context can be seen in the final episodes, in which the PSTs took advantage of area imagery to create an evolving series of partial products strategies for performing multiplication.

\section{Final Episodes: Co-emergence of General Strategies through the Area Model}

The final episodes highlight the central role of an area interpretation of multiplication supporting the emergence of a general partial products model. Within the first three tasks of the task sequence, PSTs were asked to think about specific multiplication problems ( $3_{\text {five }} \times 23_{\text {five }}, 10_{\text {five }} \times 23_{\text {five }}$, and a $42_{\text {five }}$ x $31_{\text {five }}$ rectangle). For the fourth task of the sequence, the PSTs were asked to create a general strategy to multiply any two base five numbers (see Task 4, Figure 5-5). Initially the context of rectangles was retained in order to promote leveraging area as a visual tool to support the emerging partial products strategy. By the end of the task sequence, PSTs were able to create general strategies that did not require drawing a rectangle.

In both cycles, the PSTs began the Box of Rectangles task by selecting specific rectangles and finding the length, width, and area of those rectangles using a partial products approach. The PSTs were then able to record a general strategy that reflected their work with specific rectangles. In this section of the results, I focus on the evolving 
Investigating Alternate Numeration Systems

nature of PSTs' partial products model for multiplication, highlighting how the PSTs' mathematical activity, and my analysis of their activity, relied heavily on the area model as a tool to visualize partial products.

The PSTs relied on a variety of different example rectangles, within each teaching experiment. Therefore the evidence presented below includes a number of different examples. Figure 5-17 provides a summary of the various student strategies using a single example, making the differences between strategies more clear.

Strategy 1: Breaking into base five units. One of the earliest partial products strategies PSTs were able to leverage involved splitting an area image into smaller sections so that each flat, long, and unit became its own partial product. This initial partial products strategy co-emerged alongside PSTs' model of the meaning of multiplication as area in Episodes 3 and 4. An example of this can be seen in Figure 5-13b, where $42_{\text {five }} \times 31_{\text {five }}$ is split into 24 separate regions. Each separate region can be calculated by multiplying the appropriate subsections of the length and width. While this strategy makes each partial product rather simple to calculate, it has the disadvantage of creating many smaller problems to organize. A second example of this strategy can be seen in Figure 5-17a, where $12_{\text {five }}$ X $23_{\text {five }}$ is split into 15 distinct sections.

Strategy 2: Rows of partial products. A second general strategy leveraged the area representation by splitting the area into a series of horizontal chunks (see Figure 5-15). The PSTs' description of their partial products strategy relied on area language, with the first factor corresponding to the height and the second factor to the length. The choice to leave the second factor intact while splitting the first factor "into its parts" 
created a more efficient partial products strategy than the strategy highlighted in Episode 4 (which created 24 partial products).

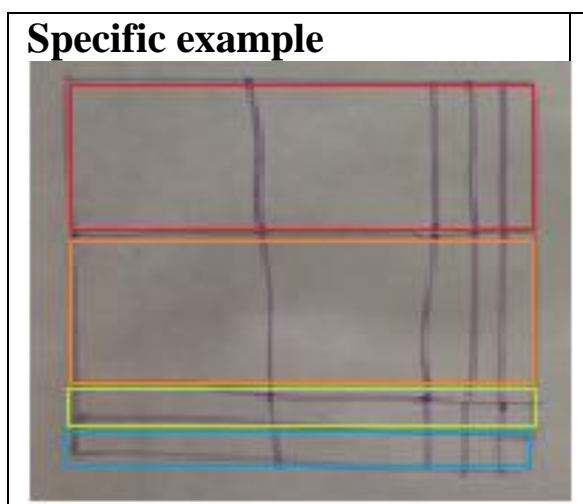

a) $22_{\text {five }} \mathrm{x} 23_{\text {five }}$ was split into 4 rows of partial products. Color added by researcher.

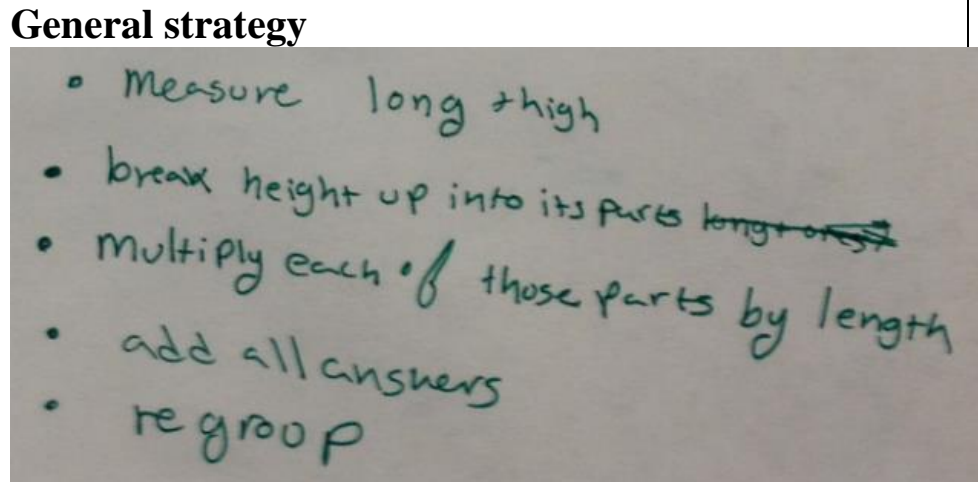

b) The general strategy created by the PSTs is closely tied to the area model language.

Figure 5-15. Nora and Sarah's general strategy for partial products based on rows

Strategy 3: Quadrants of partial products. The PSTs demonstrated another way to leverage the area representation, splitting the rectangle into quadrants (Figure 5-16). There were two key developments present in this strategy. First, similar to Strategy 2, the height was split along place value lines, but unlike Strategy 2, matching place value elements were kept together. For example, in Strategy 3 (Figure 5-16) the number $34_{\text {five }}$ was split into two sections, $30_{\text {five }}$ and 4 five, rather than 7 sections ( 3 separate longs and 4 separate ones) as it would be for Strategy 2. The second key development involved splitting both factors, rather than only one (this was part of Strategy 1, but not Strategy 2). This resulted in 4 partial products for a two digit by two digit multiplication problem. This was particularly advantageous because each partial product was relatively simple, consisting of one significant (non-zero) digit per factor rather than two or more significant digits. That is, it was easier to multiply $40_{\text {five }}$ by $30_{\text {five }}$ than to multiply $40_{\text {five }}$ by $34_{\text {five. }}$ 


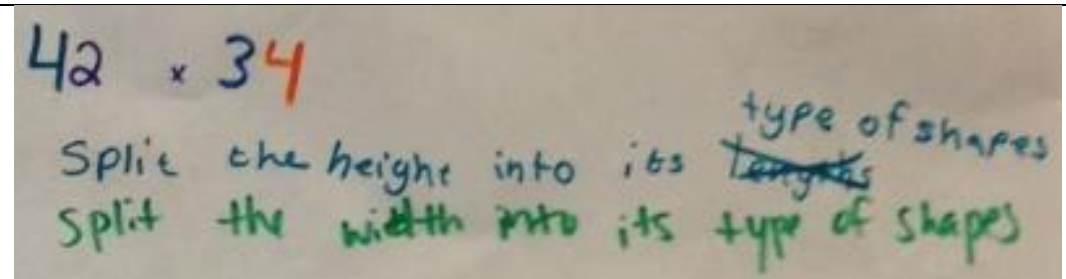

Each factor was split along place value lines. For the example of $42_{\text {five }}$ x $34_{\text {five }}, 42_{\text {five }}$ was split into $40_{\text {five }}+2$ five and $34_{\text {five }}$ was split into $30_{\text {five }}+4_{\text {five }}$.

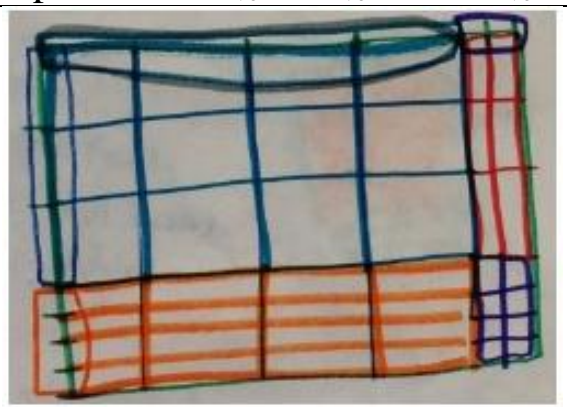

The 2 digit by 2 digit problem was split into 4 quadrants to create 4 partial products.

Figure 5-16. Nora and Sarah's written work displayed a strategy of splitting the rectangular image into quadrants to create four partial products.

The partial products strategy outlined in Figure 5-16 is closely related to the standard expanded algorithm that is often included in elementary school curriculum (see Figure 5-4). The standard algorithm also splits a 2 digit by 2 digit problem into four partial products by splitting both factors along place value lines (though it is recorded as two partial products rather than four.)

The reason I have presented three distinct strategies for multiplication is (a) to highlight the utility of the co-emerging model for the meaning of multiplication, particularly the area interpretation, as a tool for generating and visualizing the coemerging partial products strategies, and (b) to draw attention to the iterative process of refining a partial products strategy for multiplication. I do not claim that these are all the possibly strategies that might be seen as PSTs generalize a partial products strategy. Nor do I claim that each PST's model for multiplication will evolve through all of these 
levels. Rather, I argue that there are a variety of ways for partial products strategies to emerge and undergo refinement, while emphasizing the key role of the co-emerging area imagery and language in creating and sharing strategies. When teaching in a whole class setting, it may be useful to consider how to visualize and sequence various strategies to highlight increasing levels of sophistication/efficiency. Figure 5-17 displays three different partial products strategies, with distinct partial products indicated by color.

a) PSTs initially split the rectangle into many smaller sections so that each flat, long, or one can be considered its own section. In the image on the right, the problem $12 \times 23$ is split into 15 separate sections. The advantage of this partial products model is that each partial product is as simple as possible. Ex) 1 long $\mathrm{x} 1$ long = 1 flat

b) PSTs split one of the two factors along place value lines while leaving the other factor intact. In this image, $12_{\text {five }}$ is split into $10+1+1$. Similarly, PSTs could also split $12_{\text {five }}$ into $10+2$. The key distinguishing feature of this strategy is that one number is split while the other is kept whole, resulting in a collection of rows within the rectangle.

c) The partial products strategy represented at the right involves splitting each factor along place value lines. This has the advantage of having relatively few sections (unlike (a)), while also having each partial product relatively simple. Ex) 1 long $\mathrm{x} 2$ longs = 2 flats.

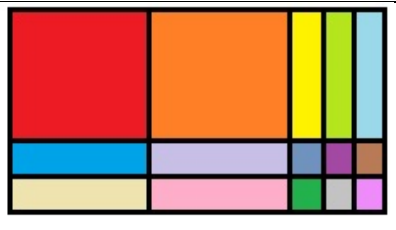

Figure 5-17. Different versions of the partial products model highlight different ways of splitting the area model into subsections.

\section{Conclusions}

One of the primary results of the study was the conclusion that PSTs' emerging model for the meaning of multiplication was still undergoing development while their partial products strategies for performing multiplication began to emerge. It may have been possible to analyze the student mathematical activity in this study by considering their mathematical activity as the emergence of a single complex model of multiplication. However, I argue that considering their activity as the co-emergence of two models (or 
two sub-models of an overarching model) provides multiple benefits. First, it simplifies the complex student activity, allowing me to consider the development of two simpler models rather than a single, more complex model. It also has explanatory power, which is a key indicator of utility. By considering the two models separately, I am able to make sense of how to leverage one emerging model to support the other. The design heuristic of co-emergence of models is both a tool for designing instructional sequences when two or more co-developing models are present and a tool for supporting teachers' in-themoment leveraging of one emergent model to advance the other. Co-emergence of models is also a research lens for analyzing student mathematical activity within those sequences. Next, I provide three examples of co-emerging models, drawn from whole numbers, linear algebra, and group theory.

1. With whole numbers, models for the meaning of place value number systems may co-emerge alongside strategies for performing addition and subtraction of multidigit numbers. Leveraging a task sequence in a base eight candy factory context, researchers have examined how PSTs create strategies for performing addition and subtraction in base eight (McClain, 2003; Yackel et al., 2007). PSTs' models for performing operations may co-emerge alongside their models for the base eight number system.

2. In linear algebra, models for operating with vectors, including geometric interpretations for combining vectors, may co-emerge alongside concepts of span and linear independence (Wawro, Rasmussen, Zandieh, Sweeney, \& Larson, 2012; Wawro, Sweeney, \& Rabin, 2011) 
Investigating Alternate Numeration Systems

3. In abstract algebra, if students are given the opportunity to reinvent groups, their models for representing group elements and their models for calculating with those elements may co-emerge as each emergent model informs the other (Larsen, 2009).

In each case, the development of a complex model can be described as the symbiotic overlap and co-emergence of multiple models. Co-emergence allows the teacherresearcher to split a complex concept into co-developing components. This allows the teacher-researcher to attend to the growth of each model somewhat independently and to recognize opportunities to leverage one partially developed model to support another developing model. 
Investigating Alternate Numeration Systems

\title{
Chapter 6 : Problematizing Mathematical Contexts
}

Problematizing Mathematical Contexts:

A Design Heuristic for Making Routine Content New Again

\begin{abstract}
Teacher educators must engage preservice teachers in developing a deeper understanding of routine mathematics. In this brief report, I introduce a design heuristic for creating tasks which allow preservice teachers to investigate a non-routine context in order to better understand a familiar context: problematizing the mathematical context. I situate this heuristic within the theory of Realistic Mathematics Education. This design heuristic provides both mathematical and pedagogical benefits by creating opportunities to deepen mathematical understanding by comparing mathematical contexts and experience the type of inquiry learning advocated by current mathematics education policy.
\end{abstract}

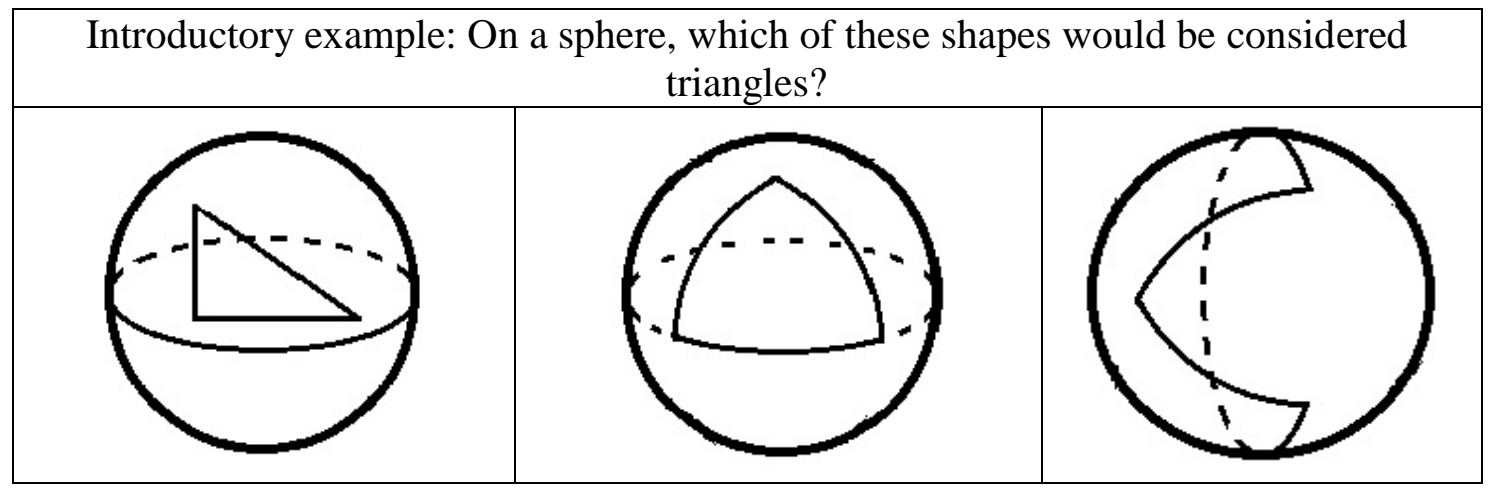

Figure 6-1. Three shapes are presented as potential examples of triangles on a sphere. Students may investigate geometry on a sphere to better understand planar geometry.

Current mathematics standards documents describe a learning environment where teachers engage students in practices of sense-making, constructing and sharing arguments, and making use of regularity and structure (National Council of Teachers of Mathematics, 2000; National Governors Association Center for Best Practices \& Council of Chief State School Officers, 2010). These standards place teachers in the position of 
supporting meaningful student inquiry which includes supporting students as they engage with rich mathematical tasks. Teacher educators are privileged with the difficult task of preparing prospective and in-service teachers to support this type of rich learning environment. This can be particularly challenging when working with content that is already familiar to preservice teachers (PSTs).

One major challenge that teacher educators face is that PSTs think they already know enough mathematics to teach elementary school (Philipp et al., 2007; Thanheiser et al., 2013). However, research indicates they lack the mathematical knowledge for teaching they will need to support children's learning (Ball et al., 2005; Ma, 1999; Thanheiser, 2009a). In order to support PSTs in developing deeper understanding of familiar content, teacher educators need tools to design learning opportunities which are (a) genuine problems ${ }^{17}$ for PSTs, (b) relate to the mathematical content that PSTs will teach, and (c) engage PSTs in the types of mathematical activity (investigation/ inquiry/ reinvention) advocated by current educational policy for mathematics.

In this brief report I establish a design heuristic which I call problematizing the mathematical context ${ }^{18}$, in which a familiar mathematical context is modified to provide a novel context for investigation and comparison to the original mathematical context (Figure 6-2).

\footnotetext{
${ }^{17}$ By genuine problems, I mean problems for which PSTs do have an accessible entry point but do not have predetermined solution strategies (Kolovou et al., 2009).

${ }^{18}$ Hiebert et al. $(1996,1997)$ use the term problematizing within problem solving literature to describe the student activity of creating solution strategies rather than practicing procedures. My use of the phrase problematizing the mathematical context focuses on task design and is not intended to serve the same purpose as Hiebert et al.'s use of the term problematizing.
} 


\section{Problematizing the mathematical context: a task design heuristic for shifting a familiar or routine mathematical context to a novel context in order to provide an opportunity for authentic mathematical investigation and comparison to the original mathematical context.}

Figure 6-2. Definition of the design heuristic problematizing a mathematical context.

In this report I situate the design heuristic of problematizing within the wider design theory of Realistic Mathematics Education. I focus on one primary example (alternate bases) and offer additional examples from non-Euclidean geometry, and group theory. Alternate base number systems are examples of problematized mathematical context because the context is mathematically similar in structure to the routine context (base ten), but the shift to a non-routine context places PSTs in a position to engage in authentic mathematical investigation of the new context and comparison to the old context. Within the examples, I highlight (a) the mathematical advantages of having a system to compare against to better understand the structure of a commonly used system and (b) the pedagogical advantages of having a novel context to explore. By pedagogical advantages, I mean opportunities for PSTs to experience the type of authentic mathematical investigation advocated by current policy standards and reflect on the learning processes that their own students may experience.

\section{Realistic Mathematics Education}

Realistic Mathematics Education (RME) is an instructional design theory which originated in the Netherlands out of a need to reform mathematics education and provide opportunities for students to engage in genuine problem solving (Gravemeijer, 1998;

Kolovou, Van den Heuvel-Panhuizen, \& Bakker, 2009). Central to RME is the idea that 
students should be provided opportunities to reinvent important mathematical ideas by leveraging their informal knowledge in contexts which are experientially real for students. RME research focuses on both curriculum development and contributions to educational theory, serving as a resource for both practitioners and researchers in the field of mathematics education.

In RME, the term experientially real refers to contexts which are familiar and make sense to students on an intuitive level (Gravemeijer, 1998), contexts in which students can engage in using common sense (Freudenthal, 1991). In order for a context to be experientially real, it does not have to be concrete or exist in daily living. The context of a made-up game or fantasy story can become experientially real to children if children can interact with the context in a commonsensical way. Also, what counts as experientially real depends on the background of the students (both mathematical background, and lived experiences). In calculus, functions can be considered experientially real because students have developed intuition about how to work with functions, including how to represent and combine functions.

Gravemeijer notes that "The RME theory is not a fixed a priori theory but is a theory that is always 'under construction.' RME is being developed in an ongoing process of designing, experimenting, analyzing, and reflecting" (Gravemeijer, 1999, pp. 157-158). Next I describe the three major RME design heuristics, guided reinvention, emergent models, and didactic phenomenology, and I describe how my proposed design heuristic extends RME theory by addressing how to work with preservice teachers in overly-familiar contexts. 
Investigating Alternate Numeration Systems

\section{RME design heuristics}

Guided reinvention is the first design heuristic of RME. Guided reinvention refers to the idea that teachers and curriculum designers should create opportunities for students to leverage their informal knowledge to construct important ideas in mathematics. Tasks should be designed to support students in making use of their intuitive knowledge in situated contexts, encouraging students to build up to more general and formal mathematics. "The idea was to allow learners to come to regard the knowledge they acquire as their own, personal knowledge, knowledge for which they themselves are responsible" (Gravemeijer \& Terwel, 2000, p. 786).

Gravemeijer describes how a task designer begins by engaging in a "thought experiment by imagining a route the students could have invented themselves" (Gravemeijer, 1999, p158). When engaged with this thought experiment it is vital for the task designer to consider the prior experience of the intended students. The reinvention routes one might create for children may not be appropriate for PSTs. PSTs have preestablished ideas, procedures, and rules within familiar domains (such as base ten and planar geometry) and so it becomes challenging to create a route for reinvention in these familiar contexts where the objective of the reinvention is an idea that already exists as a formal rule or procedure.) In these familiar contexts, prior formal mathematics can serve as a barrier to reinvention of conceptual understanding.

The emergent models design heuristic provides insight into why it is difficult to repair underlying gaps or weaknesses in PSTs' knowledge in routine contexts. The emergent models design heuristic captures how students' engage in mathematizing, beginning with work in a local or situated context where students mathematize the 
context by creating a model-of the context. The model-of a context shifts into a modelfor more formal or more general reasoning (Gravemeijer, 1999). Because PSTs have formal mathematical rules for familiar contexts they would not experience a need to mathematize those familiar situations. Vinner uses the term pseudo-problem-solving situations to capture when tasks are intended to promote conceptual behavior, i.e. behavior that is "based on meaningful learning and conceptual understanding" (1997, p.100), but which students solve by less ideal methods, such as recalling procedures. Because of their prior knowledge of rules and procedures in familiar mathematical contexts, PSTs are particularly vulnerable to leveraging their procedural knowledge without engaging in conceptual behavior. For example, if a teacher educator's goal is to support PSTs in reinventing a conceptual understanding of a multidigit multiplication strategy, then prior procedural knowledge of a standard algorithm for multiplication prevents PSTs from engaging in the desired activity of reinvention.

If PSTs already have formal mathematical rules, why is that not sufficient? Researchers have shown that PSTs have procedural knowledge that is not backed with the conceptual knowledge they will need to support children's learning (i.e. Ball, 1990b; Ball, Hill, \& Bass, 2005; Ma, 1999; Thanheiser, 2009). Moreover, this procedural knowledge acts as a barrier to developing conceptual knowledge. When advocating for guided reinvention, Freudenthal (1991) notes that

"First knowledge and ability, when acquired by one's own activity, stick better and are more readily available than when imposed by others. Second discovery can be enjoyable and so learning by reinvention may be motivating. Third it fosters the attitude of experiencing mathematics as a human activity." (p.47) 
Reinvention is an established technique for supporting students in developing knowledge that is connected to meanings (Freudenthal, 1991; Gravemeijer, 2004). However, reinvention is problematic in familiar contexts where the objective of the reinvention activity already exists in the form of known rules or procedures. In particular, PSTs have no need to mathematize a context for which they already have formal techniques.

In order to address the particular needs of PSTs, who already have access to formal mathematical rules in familiar contexts, one strategy is to shift the familiar context to a novel one. The novel context then serves as an open space for constructing or reinventing ideas, allowing PSTs to step away from their familiar (and cluttered) landscape of known procedures and rules. This purposeful shifting from familiar to nonroutine context is at the core of the problematizing design heuristic.

The design heuristic of didactic phenomenology works in conjunction with the design heuristics of guided reinvention and emergent models to inform the choice of mathematical context for investigation (Gravemeijer \& Terwel, 2000). Didactic phenomenology is the study (by the instructional designer) of how a particular context or phenomenon can be tapped for sense making and generalizing by students. The context chosen should be one that begs for organizing from a mathematical perspective (i.e. mathematizing) (Gravemeijer \& Cobb, 2006; Treffers, 1987b). Moreover, the type of mathematizing that students naturally engage in should be the type of mathematizing intended by the instructional designer (Gravemeijer, 1998). The contexts chosen by the instructional designer may be historic in nature, such as contexts that led to key developments in mathematics, or they may be common place contexts that embody a mathematical phenomenon. The context must be experientially real to students so that 
they can leverage their own intuition as a starting point for informal strategies. Nonroutine contexts may initially appear to be in contradiction to the notion of experientially real, as the non-routine contexts are often unfamiliar to the PSTs. However, the concept of experientially real captures the idea that mathematics should begin with common sense and continue with common sense (Freudenthal, 1991; Gravemeijer, 1998). For example, calculating in base ten does not tap into common sense because PSTs rely on their (potentially) superficial knowledge of memorized procedures rather than reasoning about the context. Therefore, shifting to an alternate base context is actually more experientially real for PSTs because it prompts reasoning with common sense rather than memorized rules.

The claim that non-routine contexts can be more experientially real than familiar contexts does require some additional comment. In order to use common sense thinking in an unfamiliar context, PSTs should be provided with opportunities to explore the novel context. Visual models or manipulatives often serve as an entry point for the non-routine contexts. Examples include place value manipulatives to prompt engagement with alternate bases and transparent spheres to provide an access point to spherical geometry. Initial tasks within these novel contexts should focus on drawing out PSTs' intuition for the contexts and providing situated problems for PSTs to begin to mathematize. Consider the example of multiplication in base five. Providing PSTs with base five manipulatives allows them leverage their intuition about repeated addition to reason about multiplication and to connect repeated addition and area representations. The intention of the manipulatives is to support commonsensical reasoning. 
Investigating Alternate Numeration Systems

I consider the design heuristic of problematizing the mathematical context to be a special case of didactic phenomenology that can be used in task design situations where students have prior procedural knowledge standing in place for conceptual understanding.

In the next section, I discuss alternate bases as an example of a problematized mathematical context. After this in-depth example, I also provide examples of problematizing from non-Euclidean geometry and group theory. Then I summarize and conclude by highlighting directions for future research on the role of problematizing in PST education.

\section{Examples}

\section{Primary Example: Alternate bases}

Alternate bases are a commonly used tool in PST content courses. Both historic and modern alternate bases systems appear in PST textbooks (e.g., Bassarear, 2012; Bennett, Burton, \& Nelson, 2012). Alternate bases have been used in a variety of ways to research PSTs' understanding of whole number and operation. For example, researchers have used alternate bases to explore place value understanding, addition, and subtraction (McClain, 2003; Thanheiser, 2015a; Yackel et al., 2007); multidigit multiplication (Fasteen et al., 2015); and decimal fraction understanding (Zazkis \& Khoury, 1993). In this section I present an example of a task in the problematized context of base five after providing a brief introduction to base five.

The base five number system is a place value number system where each place corresponds to a power of five. There is a ones place, a fives place, and twenty-fives place, and so on. Consider the example $432_{\text {five. }}$. The 4 refers to 4 groups of twenty-five, the 3 refers to 3 groups of five, and the 2 signifies 2 ones. The base five number system 
is similar to base ten in that it takes "base" copies of a unit to regroup to create one of the next larger unit. Because the quantity at which students regroup is different from the routine base ten system, students must pay particular attention to when and how to regroup and how that affects the overall structure of the number system.

\section{Times Base Task}

- Specific example: Find the product $10_{\text {five }} \times 23_{\text {five }}$ and explain your reasoning.

- Generalizing in base five: Make a conjecture about multiplying by $10_{\text {five }}$. Justify your conjecture.

- Generalizing across systems:

Compare across bases: $10 \times 23=230$ and $10_{\text {five }} \times 23_{\text {five }}=230_{\text {five }}$. Is this a coincidence or something more?

Figure 6-3. The Times Base Task provides an opportunity to consider the multiplicative structure of place value systems.

Figure 6-3 contains an example of an alternate base task which focuses PSTs' attention on making sense of the structure of a place value number system. In this task, PSTs are asked to examine multiplication by $10_{\text {five }}$ in base five. That is, they are asked to examine multiplication by the quantity that looks like ten and to explore how and why the quantity $10_{\text {five }}$ behaves like ten when multiplying. Details about PSTs' mathematical activity with this task can be found in Fasteen et al. (2015). The problematized context of base five provides both mathematical and pedagogical advantages.

1) Mathematical advantages: Because of the common structure of base five and base ten, PSTs may be able to make generalizations beyond specific bases, generalizing across number systems. For example, PSTs may be able to recognize and justify why multiplication by $10_{\text {base }}$ causes a zero to be appended to a number regardless of the base. Making sense of what causes digits to shift to the left in base five may help PSTs to make sense of this same phenomenon in base ten. 
Investigating Alternate Numeration Systems

2) Pedagogical advantages: PSTs do not have prior memorized rules for working with base five, as they do for base ten. For example, in base ten, PSTs likely know that multiplying by 10 causes a zero to be appended to a number, but they do not have a similar pre-established rule in base five. Therefore, PSTs have opportunities to engage in the types of inquiry and reinvention advocated by reform, including discovering patterns and creating general algorithms for multidigit operations (Fasteen et al., 2015; McClain, 2003; Yackel et al., 2007).

It is important to recognize that the goal of investigating an alternate numeration system is rarely to better understand that specific number system or to become skilled at quickly solving problems in that system. Rather, the goal of investigating alternate numeration systems is to better understand the structure of place value systems (including the routine base ten (Chapter 4)) and to experience the process of creating sense-making strategies based upon that structure.

From the task designer's perspective, alternate bases like base five provide an opportunity to vary a key aspect of our routine base ten number system, namely the value at which regrouping occurs in the number system. According to variation theory, it is by varying aspects that we draw attention to them (M. L. Lo, 2012), so the use of an alternate base draws attention to the underlying structure of place value systems. Likewise, historic number systems, such as Ancient Egyptian numerals, provide a context for altering the structure from a place value system (where the location of a digit indicates its value) to an additive system (where a value is determined by summing the value of each symbol) (Thanheiser, 2015b). Alternate numeration systems, both historic 
and modern, can serve as problematized mathematical contexts for base ten because they serve the dual role of providing a context which supports authentic mathematical investigation and a novel context to compare against a routine context.

\section{Additional Examples}

Base ten is not the only routine context that PSTs and teacher educators' encounter. Geometry and properties of operations are also topics which PSTs have seen across their K-12 experiences. Next I provide examples related to the routine contexts of (1) Euclidean geometry and (2) the associative and commutative properties.

Geometry. The problematized context of non-Euclidean geometry is common to secondary PST content courses in geometry (Grover \& Connor, 2000). Non-Euclidean geometry includes spherical geometry, which provides a contrast to planar geometry and can serve as a non-routine context to explore defining, conjecturing, and justifying (Zandieh \& Rasmussen, 2010), as well as taxicab geometry, which can be used to focus attention on measuring distance by altering the way in which distance is calculated (Siegel, Borasi, \& Fonzi, 1998). The design heuristic of problematizing the mathematical contexts focuses on leveraging these non-routine geometries for both mathematical and pedagogical purposes. Consider the introductory example about triangles on a sphere. PSTs have known what a triangle was since they were young children. The spherical context places them in a position where understanding a triangle becomes a genuine problem, a problem which requires mathematizing the spherical context. In addition to this pedagogical benefit of having a context to explore and mathematize, non-Euclidean geometries provide a context to compare against Euclidean geometry. This can help 
PSTs to step back from the familiar planar geometry of their prior experience and consider this as an example of a wider phenomenon.

Associative and Commutative Properties. Larsen (2010) provides insight into a problematized mathematical context for revisiting the ideas of associativity and commutativity. The associative and commutative properties capture two different ways in which the "order" of numbers can be altered without changing the total value. The associative property (of addition) states that the order in which numbers are grouped does not affect their sum $($ so $a+(b+c)=(a+b)+c)$, while the commutative property (of addition) states that the order in which numbers are placed in an addition problem does not affect their sum (so $a+b=b+a)$. PSTs often confound these two properties (Ding, Li, \& Capraro, 2013; Zaslavsky \& Peled, 1996). Larsen investigated student mathematical activity with these properties in the non-routine context of symmetries of an equilateral triangle. Because symmetries of an equilateral triangle form a non-commutative group, the students were able to examine a context where only one of the two properties held. This enabled them to tease apart the difference in the conceptual meanings of these properties. As with alternate bases and non-Euclidean geometries, by varying some aspect of the routine context, PSTs can be placed in a problematized mathematical context where they can engage in authentic mathematical investigation.

\section{Summary \& Conclusions}

Problematizing a mathematical context is a design heuristic that is intended to support teacher educators in creating tasks which serve as genuine problems for PSTs. Shifting to problematized contexts creates an opportunity for PSTs to experience routine content, such as whole number operations, in a new way. PSTs can engage in inquiry 
tasks because the novel context allows them to set aside their prior rules and procedures and focus on sense making in the novel context. The problematized contexts also provide opportunities for comparison of mathematical structures because they leverage variations in the structure of the routine contexts. The use of variation draws attention to attributes of the routine context that might otherwise go unnoticed, such as the meaning of a line in geometry or the role of zero in place value systems.

\section{Directions for future research}

Problematizing mathematical contexts is something that is already done in teacher education and it provides multiple advantages for teacher educators as they work with PSTs. In naming this design heuristic, I am drawing attention to it both as a tool for curriculum design and as a theoretical contribution which would benefit from further research. I conclude with a call for future research, highlighting additional questions about problematizing which bear further investigation.

While I have provided examples from whole number and operation, properties of operation, and geometry, I anticipate that there are additional mathematical contexts which might benefit from problematizing. Future research on problematizing mathematical contexts should consider what mathematical contexts might benefit from problematizing (beyond whole numbers and geometry).

Within this report I have focused on the benefits of problematizing mathematical contexts for work with PSTs. Additional research should address both the affordances and constraints of this approach to advancing PSTs mathematical knowledge. While I have focused this report on work with PSTs, there may be related reasons to leverage problematizing for students who are not PSTs. This begs the questions: When is it most 
Investigating Alternate Numeration Systems

appropriate and impactful to problematize contexts? How/why do mathematicians leverage problematized mathematical contexts? And how is that different from how/why teacher educators leverage problematizing? 
Investigating Alternate Numeration Systems

\section{Chapter 7 : Conclusions}

Alternate numeration systems are a common context leveraged in curriculum (e.g. Bassarear, 2012c; Bennett, Burton, \& Nelson, 2012a) and research (e.g. Fasteen, Melhuish, \& Thanheiser, 2015; McClain, 2003; Yackel et al., 2007; Zazkis \& Khoury, 1994) related to PSTs' understanding of whole number and operation. The intention of my dissertation was to investigate the role of alternate numeration systems in supporting preservice teachers' (PSTs) development of mathematical knowledge, particularly mathematical knowledge for teaching (Ball et al., 2005). I approached this investigation in three distinct ways. First, I investigated alternate numeration systems in existing curricula, finding both mathematical and pedagogical affordances of alternate numeration systems. Second, I approached alternate bases by conducting a teaching experiment leveraging base five and using the design principles of RME. In this teaching experiment, I demonstrated that PSTs were able to reinvent a general strategy for multidigit multiplication in base five. This provided a context for the PSTs to develop a deeper understanding of multidigit multiplication, as well as an opportunity for them to experience the reinvention process. My third approach to the context of alternate numeration systems was to consider it as an example of a wider phenomenon of problematizing routine mathematical contexts. I argued for problematizing mathematical contexts as a design heuristic, situating this within the design theory of Realistic Mathematics Education (RME) (Freudenthal, 1991; Gravemeijer, 1998), particularly within the design heuristic of didactic phenomenology.

These three approaches to investigating the role of alternate numeration systems allowed me to examine alternate numeration systems at three distinct levels. I was able 
Investigating Alternate Numeration Systems

to analyze the current state of alternate numeration systems (as they appear in textbooks) as a tool to support PSTs' in developing a stronger understanding of whole number and operation. I was then able to narrow in on a particular subtopic within whole number and operation (multiplication) and examine PSTs' mathematical activity as they made sense of multidigit multiplication in base five. I was also able to expand out from the topic of whole number and operation to consider leveraging alternate bases as an example of a wider design heuristic of shifting from routine to non-routine mathematical contexts.

Reflecting back across the three studies, key contributions to research and teacher education focus on three major ideas; mathematical structure, opportunities for guided reinvention, and opportunities for PST reflection. I discuss each of these contributions below.

\section{Mathematical Structure}

Alternate numeration systems can be leveraged to focus PSTs' attention on mathematical structure, seeing base ten as an example of a wider phenomenon. Just as a native English speaker can learn more about English by studying another language, an adult who is familiar with base ten can learn more about its structure by comparing it to another number system. Lo (2012) captures this idea in her discussion of variation theory, stating that "to discern previously taken for granted features of familiar situations, learners must experience for themselves certain patterns of variation and invariance of these features" (p. 83). In the textbook analysis (Chapter 4), this focus on comparing to better understand structure was one of the dominant themes for alternate numeration systems across the curricula. Both historic numeration systems and modern alternate base systems were utilized for this purpose. 
Investigating Alternate Numeration Systems

An exampling of focusing on mathematical structure can be seen in the teaching experiment for multidigit multiplication (Chapter 5). PSTs were able to step back from the tasks of multiplying by ten or $10_{\text {five }}$ and recognize that multiplying by $10_{\text {base }}$ in any base would have the same effect of shifting digits to the left. They were able to make sense of and leverage the multiplicative structure which is common to the base ten and base five number systems.

In the brief report on problematizing mathematical contexts (Chapter 6), I discussed leveraging alternate bases as an example of a wider phenomenon of leveraging problematized mathematical contexts. One of the primary affordances of problematizing mathematical contexts is to allow for comparison to the structure of the routine mathematical context. Task designers can focus on key structural properties that are similar or different between the routine context and the problematized context. For example, base ten uses a place value structure, while the Egyptian numeration system does not. This focus on varying key attributes of a mathematical idea can be seen in Lo's (2012) work on variation theory and Thanheiser's (2015b) use of variation theory in task design with historic numeration systems.

\section{Opportunities for Guided Reinvention}

Guided reinvention is a design heuristic for supporting students as they recreate important ideas in mathematics (Freudenthal, 1991; Gravemeijer, 1998). Alternate bases can provide an opportunity for guided reinvention of algorithms as seen in the teaching experiment in Chapter 4 and in prior research (Fasteen et al., 2015; McClain, 2003; Yackel et al., 2007). These reinvention opportunities can promote a shift in PSTs' view of elementary mathematics, helping them to see mathematics as a sense-making endeavor 
rather than a series of rules to practice (McClain, 2003). However, my analysis of PST textbooks indicates that opportunities for guided reinvention are limited. Other than the Bassarear (2012a) curriculum, which provides an activity for PSTs to reinvent a base five number system, the curricula generally focused on providing solution strategies rather than posing tasks which would press the PSTs to invent strategies.

Teacher educators may want to consider creating opportunities for PSTs to engage in guided reinvention. Researchers have found that children are capable inventors of multidigit strategies (Ambrose et al., 2003; Fuson et al., 1997; van Putten et al., 2005), provided they have the support of capable teachers. PSTs may not have had an opportunity to engage in reinvention of algorithms in their own elementary math experiences, but teacher educators may be able to provide those learning opportunities by leveraging alternate bases. My instructional sequence provides an example of how to support guided reinvention of an algorithm for multiplication in base five. One key finding of this study was that PSTs' reinvention of strategies for multiplication coemerged alongside their developing models for the meaning of multiplication. McClain (2003) and Yackel et al. (2007) offer examples of task sequences to support guided reinvention of addition and subtraction in base eight. Together, these studies provide a collection of resources for supporting PSTs in engaging in meaningful reinvention of core ideas related to whole number and operation. The design heuristic of problematizing mathematical contexts provides insight into creating additional contexts which support guided reinvention, both in and beyond the content area of whole number and operation. 
Investigating Alternate Numeration Systems

\section{Opportunities for Reflection}

The reform movement asks teachers to teach in ways they may not have experienced as children. As adult learners, alternate bases provide an opportunity for PSTs to experience the types of inquiry based instruction that reform advocates. In order for these experiences to have the greatest impact, PSTs should have opportunities to reflect on their learning experiences, both in the moment and retrospectively.

Adults may not recall how difficult it was to learn how to count in kindergarten or first grade. They may not recall a time when they didn't know that the number after 99 is 100. While learning a new number system, such as base five, PSTs can engage with discoveries that mirror the discoveries their future students will experience. For example, when multiplying in base five, PSTs can make the discovery that multiplying a number by $10_{\text {five }}$ causes all of the digits in that number to shift to the left. This mirrors the discovery children make about multiplying by ten. Exploration of multiplication by $10_{\text {five }}$ can provide an opportunity for PSTs to make sense of the multiplicative structure of place value systems. Reflection on this learning process can help PSTs to see elementary mathematics as a sense-making endeavor, rather than a set of predetermined rules.

Likewise, the process of inventing a strategy for an operation can help PSTs to understand the process of creating a general algorithm. Reflecting on reinvention can focus attention on the process of building up from less efficient to more efficient invented strategies. It can also help PSTs to value the use of visual models and manipulatives to support emergent strategies, rather than relying entirely on symbolic representations.

McClain (2003) found that PSTs struggled to engage in making sense of the mathematics while simultaneously reflecting on the learning process of children. She 
found it helpful to create opportunities for reflection after PSTs had engaged in the learning process. In my own teaching, including the teaching experiment for multiplication, I have found that PSTs may engage in spontaneous reflection as they encounter struggles and triumphs and make comments on their own learning process. As captured by the Bassarear (2012c) curriculum "I wish I had a dollar for every student who has said something like 'Wow, no wonder it's hard for little kids to learn how to count; I never thought of it [our system] that way before" (p. 102). Opportunities for both spontaneous and retrospective reflection may support PSTs in making the most of non-routine learning opportunities.

My study adds to the existing body of research on alternate numeration systems, focusing on how this non-routine context can be leveraged to support PSTs. While this study answered some research questions, it also raised several more. In the final section, I discuss future directions for research.

\section{Future Research}

The research project represented in this dissertation could be seen as three separate studies investigating three separate aspects of alternate numeration systems in PST content courses. This dissertation can also be seen as part of a larger conversation about the current role of problematized mathematical contexts in supporting PST education. I conclude my study by considering further directions for research related to the problematized context of alternate numeration systems and their role is PST mathematics courses. 
Investigating Alternate Numeration Systems

- The investigation of rationales for leveraging alternate systems could be expanded beyond the written curricula to include a large scale look at why teacher educators choose to include (or exclude) alternate systems in their classrooms.

- In addition to considering why alternate systems might be included, research could expand on additional ways in which alternate systems are leveraged in the classrooms, beyond the tasks that appear in written curricula. The curriculum that occurs in live classrooms is often different than the curriculum that appears on the written page (Stein et al., 2007).

- Additional teaching experiments are needed to better understand how different alternate numeration system activities promote different types of student mathematical activity. This could include a larger scale implementation of the multiplication task sequence, as well as investigations of other potential task sequences.

- The role of problematizing of mathematical contexts could be investigated in contexts other than alternate numeration systems. Non-Euclidean geometry serves as another example of a problematized mathematical context. Research in the problematized context of alternate bases could benefit research in the problematized context of non-Euclidean geometry, and vice versa.

- While my study has highlighted benefits of leveraging problematized mathematical contexts, an additional avenue for research may focus on hindrances of problematizing. Challenges of alternate bases may focus around the additional specialized knowledge need by teacher educators, the classroom time commitment, and the potential that PSTs view the non-routine context as artificial 
or extraneous. An investigation of why teacher educators chose to include or exclude alternate systems may be a valuable starting point to consider potential hindrances.

- Additional research may examine what supports teacher educators need in order to successfully implement innovative curricula, particularly curricula that they themselves have not previously experienced. Is the availability of a written curriculum (and maybe a teacher's manual) sufficient support for a teacher educator? Masingila, Olanoff, and Kwaka (2012) report that most institutions lack opportunities for professional development for the instructors of PST mathematics courses. Research could examine what types of support or professional development opportunities would offer the greatest benefit for teacher educators. 
Investigating Alternate Numeration Systems

\section{References}

Ambrose, R., Baek, J. M., \& Carpenter, T. P. (2003). Children's invention of multidigit multiplication and division algorithms. The Development of Arithmetic Concepts and Skills: Constructive Adaptive Expertise, 307.

Armanto, D. (2008). Teaching and learning multiplication of multi-digit numbers in realistic mathematics education (RME). Teaching and Learning Multiplication of Multi-Digit Numbers in Realistic Mathematics Education (RME). Retrieved from http://digilib.unimed.ac.id/UNIMED-Journal-208/21471

Ball, D. L. (1988). Knowledge and reasoning in mathematical pedagogy: Examining what prospective teachers bring to teacher education. Michigan State University. Retrieved from http://wwwpersonal.umich.edu/ dball/books/DBall_dissertation.pdf

Ball, D. L. (1990a). Prospective Elementary and Secondary Teachers' Understanding of Division. Journal for Research in Mathematics Education, 21(2), 132-144. http://doi.org/10.2307/749140

Ball, D. L. (1990b). The Mathematical Understandings That Prospective Teachers Bring to Teacher Education. The Elementary School Journal, 90(4), 449-466.

Ball, D. L., \& Cohen, D. K. (1996). Reform by the Book: What Is: Or Might Be: The Role of Curriculum Materials in Teacher Learning and Instructional Reform? Educational Researcher, 25(9), 6-14. http://doi.org/10.2307/1177151

Ball, D. L., Hill, H., \& Bass, H. (2005). Knowing mathematics for teaching: Who knows mathematics well enough to teach third grade, and how can we decide? American Educator, 14-17, 20-22, 43-46.

Ball, D. L., Thames, M. H., \& Phelps, G. (2008). Content Knowledge for Teaching: What Makes It Special? Journal of Teacher Education, 59(5), 389-407. http://doi.org/10.1177/0022487108324554

Bassarear, T. (2012a). Explorations: Mathematics for Elementary School Teachers (5th ed.). Brooks Cole, Cengage Learning.

Bassarear, T. (2012b). Instructor's Manual: Mathematics for Elementary School Teachers (5th ed.). Brooks Cole, Cengage Learning.

Bassarear, T. (2012c). Mathematics for Elementary School Teachers (5th ed.). Brooks Cole, Cengage Learning. 
Investigating Alternate Numeration Systems

Baturo, A., \& Nason, R. (1996). Student teachers' subject matter knowledge within the domain of area measurement. Educational Studies in Mathematics, 31(3), 235268.

Beckmann, S. (2014). Mathematics for Elementary Teachers with Activity Manual (4th ed.). Pearson.

Bennett, A., Burton, L., \& Nelson, T. (2012a). Mathematics for Elementary Teachers: A Conceptual Approach (9th ed.). McGraw-Hill Science/Engineering/Math.

Bennett, A., Burton, L., \& Nelson, T. (2012b). Mathematics for Elementary Teachers: An Activity Approach (9th ed.). McGraw-Hill Science/Engineering/Math.

Billstein, R., Libeskind, S., \& Lott, J. (2012). A Problem Solving Approach to Mathematics for Elementary School Teachers (11th ed.). Pearson.

Bowers, J. (1995). An alternative perspective for developing a mathematical microworld. In The first international conference on Computer support for collaborative learning (pp. 27-31). Retrieved from http://dl.acm.org/citation.cfm?id=222048

Braun, V., \& Clarke, V. (2006). Using thematic analysis in psychology. Qualitative Research in Psychology, 3(2), 77-101.

Carraher, T. N., Carraher, D. W., \& Schliemann, A. D. (1985). Mathematics in the streets and in schools. British Journal of Developmental Psychology, 3(1), 21-29.

Carroll, W. M. (1999). Invented computational procedures of students in a standardsbased curriculum. The Journal of Mathematical Behavior, 18(2), 111-121.

Cobb, P., \& Whitenack, J. W. (1996). A method for conducting longitudinal analyses of classroom videorecordings and transcripts. Educational Studies in Mathematics, 30(3), 213-228.

Cobb, P., \& Yackel, E. (1996). Constructivist, emergent, and sociocultural perspectives in the context of developmental research. Educational Psychologist, 31 (3 \& 4), 175-190.

Common Core Writing Team. (2011). The NBT Progression for the Common Core State Standards.

Creswell, J. W. (1998). Qualitative inquiry and research design: choosing among five traditions. Thousand Oaks, Calif.: Sage Publications. 
Ding, M., Li, X., \& Capraro, M. M. (2013). Preservice elementary teachers' knowledge for teaching the associative property of multiplication: A preliminary analysis. The Journal of Mathematical Behavior, 32(1), 36-52. http://doi.org/10.1016/j.jmathb.2012.09.002

Ebby, C. B. (2005). The powers and pitfalls of algorithmic knowledge: a case study. The Journal of Mathematical Behavior, 24(1), 73-87.

Fasteen, J., Melhuish, K., \& Thanheiser, E. (2015). Multiplication by the base: Making sense of place value structure through an alternate base. Mathematics Teacher Educator.

Fischbein, E., Deri, M., Nello, M. S., \& Marino, M. S. (1985). The role of implicit models in solving verbal problems in multiplication and division. Journal for Research in Mathematics Education, 3-17.

Freitag, M. A. (2012). Mathematics for Elementary School Teachers, 1st ed. Cengage Learning.

Freudenthal, H. (1991). Revisiting Mathematics Education: China Lectures. Retrieved from http://www.eric.ed.gov/ERICWebPortal/recordDetail?accno=ED341576

Fuson, K. C. (1990). A Forum for Researchers. Issues in Place-Value and Multidigit Addition and Subtraction Learning and Teaching. Journal for Research in Mathematics Education, 21(4), 273-80.

Fuson, K. C. (1992). Research on whole number addition and subtraction. In D. A. Grouws (Ed.), Handbook of research on mathematics teaching and learning (pp. 243-275). New York: Macmillan.

Fuson, K. C. (2003). Toward computational fluency in multidigit multiplication and division. Teaching Children Mathematics, 9(6), 300-305.

Fuson, K. C., \& Beckmann, S. (2012). Standard Algorithms in the Common Core State Standards. NCSM Journal, (Fall/Winter 2012-2013). Retrieved from http://www.mathedleadership.org/docs/resources/journals/NCSMJournal_ST_Alg orithms_Fuson_Beckmann.pdf

Fuson, K. C., Wearne, D., Hiebert, J. C., Murray, H. G., Human, P. G., Olivier, A. I., ... Fennema, E. (1997). Children's conceptual structures for multidigit numbers and methods of multidigit addition and subtraction. Journal for Research in Mathematics Education, 130-162. 
Graeber, A. O. (1999). Forms of Knowing Mathematics: What Preservice Teachers Should Learn. Educational Studies in Mathematics, 38(1/3), 189-208.

Graeber, A. O., Tirosh, D., \& Glover, R. (1989). Preservice teachers' misconceptions in solving verbal problems in multiplication and division. Journal for Research in Mathematics Education, 20(1), 95-102.

Gravemeijer, K. (1998). Developmental research as a research method. In Mathematics education as a research domain: A search for identity (pp. 277-295). Springer. Retrieved from http://link.springer.com/chapter/10.1007/978-94-011-5470-3_18

Gravemeijer, K. (1999). How emergent models may foster the constitution of formal mathematics. Mathematical Thinking and Learning, 1(2), 155-177.

Gravemeijer, K. (2004). Local instruction theories as means of support for teachers in reform mathematics education. Mathematical Thinking and Learning, 6(2), 105128.

Gravemeijer, K., \& Cobb, P. (2006). Design research from a learning design perspective. Educational Design Research, 17-51.

Gravemeijer, K., \& Doorman, M. (1999). Context Problems in Realistic Mathematics Education: A Calculus Course as an Example. Educational Studies in Mathematics, 39(1-3), 1-3.

Gravemeijer, K., \& Terwel, J. (2000). Hans Freudenthal: a mathematician on didactics and curriculum theory. Journal of Curriculum Studies, 32(6), 777-796.

Grover, B. W., \& Connor, J. (2000). Characteristics of the college geometry course for preservice secondary teachers. Journal of Mathematics Teacher Education, 3(1), $47-67$.

Harel, G. (2007). The DNR system as a conceptual framework for curriculum development and instruction. Foundations for the Future in Mathematics Education, 263-280.

Hiebert, J., Carpenter, T. P., Fennema, E., Fuson, K., Human, P., Murray, H., ... Wearne, D. (1996). Problem solving as a basis for reform in curriculum and instruction: The case of mathematics. Educational Researcher, 25(4), 12-21.

Hiebert, J., Carpenter, T. P., Fennema, E., Fuson, K., Human, P., Murray, H., ... Wearne, D. (1997). Rejoinder: Making Mathematics Problematic: A Rejoinder to Prawat and Smith. Educational Researcher, 26(2), 24-26. http://doi.org/10.2307/1176035 
Investigating Alternate Numeration Systems

Hiebert, J., \& Lefevre, P. (1986). Conceptual and procedural knowledge: The case of mathematics. In Conceptual and procedural knowledge in mathematics: An introductory analysis (pp. 1-23). New Jersey: Lawrence Erlbaum Associates Inc.

Hill, H. C., Ball, D. L., \& Schillig, S. (2008). Unpacking Pedagogical Content Knowledge: Conceptualizing and Measuring Teachers' Topic-Specific Knowledge of Students. Journal for Research in Mathematics Education, 39(4), $372-400$.

Hill, H., Umland, K., Litke, E., \& Kapitula, L. R. (2012). Teacher Quality and Quality Teaching: Examining the Relationship of a Teacher Assessment to Practice. American Journal of Education, 118(4), 489-519.

Hopkins, T. M., \& Cady, J. A. (2007). What is the value of @*\#? Deepening Teachers’ Understanding of Place Value. Teaching Children Mathematics, 13(8), 434-437.

Iszak, A. (2004). Teaching and learning two-digit multiplication: Coordinating analyses of classroom practices and individual student learning. Mathematical Thinking and Learning, 6(1), 37-79.

Johnson, E. (2013). Realistic Mathematics Education Design Heuristics and Implications for Documenting Student Learning. Portland State University, Portland, OR.

Kamii, C. (1994). Young children continue to reinvent arithmetic--3rd grade: implications of Piaget's theory. New York: Teachers College Press.

Kamii, C. (2000). Young children reinvent arithmetic: implications of Piaget's theory. New York: Teachers College Press.

Kamii, C., \& Dominick, A. (1997). To teach or not to teach algorithms. The Journal of Mathematical Behavior, 16(1), 51-61. http://doi.org/10.1016/S07323123(97)90007-9

Khoury, H. A., \& Zazkis, R. (1994). On Fractions and Non-Standard Representations: Pre-Service Teachers' Concepts. Educational Studies in Mathematics, 27(2).

Kilpatrick, J., Swafford, J., \& Findell, B. (2001). Adding + it up: Helping children learn mathematics. National Academies Press.

Klein, A. S., Beishuizen, M., \& Treffers, A. (1998). The Empty Number Line in Dutch Second Grades: Realistic versus Gradual Program Design. Journal for Research in Mathematics Education, 29(4), 443-464. http://doi.org/10.2307/749861 
Investigating Alternate Numeration Systems

Kolovou, A., Van den Heuvel-Panhuizen, M., \& Bakker, A. (2009). Non-routine problem solving tasks in primary school mathematics textbooks-A needle in a haystack. Mediterranean Journal for Research in Mathematics Education, 8(2), 31-68.

Lampert, M. (1986). Knowing, Doing, and Teaching Multiplication. Cognition and Instruction, 3(4), 305-342.

Larsen, S. (2004). Supporting the guided reinvention of the concepts of group and isomorphism: A developmental research project (Doctoral dissertation, Arizona State University, 2004) Dissertation Abstracts International. B, 65(02), 781.

Larsen, S. (2009). Reinventing the concepts of group and isomorphism: The case of Jessica and Sandra. The Journal of Mathematical Behavior, 28(2-3), 119-137. http://doi.org/10.1016/j.jmathb.2009.06.001

Larsen, S. (2010). Struggling to disentangle the associative and commutative properties. For the Learning of Mathematics, 30(1), 37-42.

Larsen, S. (2013). A local instructional theory for the guided reinvention of the group and isomorphism concepts. The Journal of Mathematical Behavior, 32(4), 712-725.

Lesh, R., \& Lehrer, R. (2000). Iterative refinement cycles for videotape analyses of conceptual change. Handbook of Research Design in Mathematics and Science Education, 665-708.

Lo, J.-J., Grant, T. J., \& Flowers, J. (2008). Challenges in deepening prospective teachers' understanding of multiplication through justification. Journal of Mathematics Teacher Education, 11(1), 5-22.

Lo, M. L. (2012). Variation theory and the improvement of teaching and learning. Göteborg: Acta Universitatis Gothoburgensis. Retrieved from https://130.241.16.4/handle/2077/29645

Long, C. T., DeTemple, D. W., \& Millman, R. S. (2012). Mathematical reasoning for elementary teachers (6th ed.). Pearson Addison Wesley.

Ma, L. (1999). Knowing and teaching elementary mathematics teachers' understanding of fundamental mathematics in China and the United States. Mahwah, N.J.: Lawrence Erlbaum Associates.

Masingila, J., Olanoff, D., \& Kwaka, D. (2012). Who teaches mathematics content courses for prospective elementary teachers in the United States? Results of a 
Investigating Alternate Numeration Systems

national survey. Journal of Mathematics Teacher Education, 15(5), 347-358. http://doi.org/10.1007/s10857-012-9215-2

McClain, K. (2003). Supporting Preservice Teachers' Understanding of Place Value and Multidigit Arithmetic. Mathematical Thinking \& Learning, 5(4), 281-306.

Mewborn, D. (2001). Teachers Content Knowledge, Teacher Education, and Their Effects on the Preparation of Elementary Teachers in the United States. Mathematics Teacher Education and Development, 3, 28-36.

Mullis, I. V., Martin, M. O., Beaton, A. E., Gonzalez, E. J., Kelly, D. L., \& Smith, T. A. (1997). Mathematics achievement in the primary school years: IEA's Third International Mathematics and Science Study (TIMSS). TIMSS international study center, Boston College Chestnut Hill, MA. Retrieved from http://sling11.bc.edu/timss1995i/TIMSSPDF/amtimss.pdf

Musser, G. L., Peterson, B. E., \& Burger, W. F. (2011a). Mathematics for Elementary Teachers: A Contemporary Approach (9th ed.). Wiley.

Musser, G. L., Peterson, B. E., \& Burger, W. F. (2011b). Student Activity Manual Mathematics for Elementary Teachers: A Contemporary Approach (9th ed.). Wiley.

National Council of Teachers of Mathematics. (2000). Principles and standards for school mathematics. Reston, VA.

National Governors Association Center for Best Practices \& Council of Chief State School Officers. (2010). Common Core State Standards for Mathematics. Washington,DC: National Governors Association Center for Best Practices, Council of Chief State School Officers.

Pesek, D. D., \& Kirshner, D. (2000). Interference of Instrumental Instruction in Subsequent Relational Learning. Journal for Research in Mathematics Education, 31(5), 524-540. http://doi.org/10.2307/749885

Philipp, R. A., Ambrose, R., Lamb, L. L., Sowder, J. T., Schappelle, B. P., Sowder, L., ... Chauvot, J. (2007). Effects of early field experiences on the mathematical content knowledge and beliefs of prospective elementary school teachers: An experimental study. Journal for Research in Mathematics Education, 438-476.

Rathouz, M. (2011). Visualizing Decimal Multiplication with Area Models: Opportunities and Challenges. Issues in the Undergraduate Mathematics 
Investigating Alternate Numeration Systems

Preparation of School Teachers, 2. Retrieved from

http://www.eric.ed.gov/ERICWebPortal/detail?accno=EJ962624

Ross, S. H. (2001). Pre-service elementary teachers and place value: Written assessment using a digit-correspondence task. In Proceedings of the twenty-third annual meeting of the north American chapter of the international group for the psychology of mathematics education (pp. 897-906).

Roy, G. J. (2014). Developing Prospective Teachers' Understanding of Addition and Subtraction with Whole Numbers. Issues in the Undergraduate Mathematics Preparation of School Teachers: The Journal, 2 (Pedagogy. Retrieved from http://www.k-12prep.math.ttu.edu/journal/pedagogy/roy01/article.pdf

Schifter, D., \& Fosnot, C. T. (1993). Reconstructing Mathematics Education: Stories of Teachers Meeting the Challenge of Reform. New York: Teachers College Press.

Siegel, M., Borasi, R., \& Fonzi, J. (1998). Supporting students' mathematical inquiries through reading. Journal for Research in Mathematics Education, 378-413.

Silverman, J., \& Thompson, P. W. (2008). Toward a framework for the development of mathematical knowledge for teaching. Journal of Mathematics Teacher Education, 11(6), 499-511. http://doi.org/10.1007/s10857-008-9089-5

Simon, M. A., \& Blume, G. W. (1994). Building and Understanding Multiplicative Relationships: A Study of Prospective Elementary Teachers. Journal for Research in Mathematics Education, 25(5), 472-494.

Skemp, R. R. (1978). Relational Understanding and Instrumental Understanding. The Arithmetic Teacher, 26(3), 9-15.

Southwell, B., \& Penglase, M. (2005). Mathematical knowledge of pre-service primary teachers. International Group for the Psychology of M Athematics Education, 209.

Sowder, J., Sowder, L., \& Nickerson, S. (2014). Reconceptualizing Mathematics (Second Edition). W. H. Freeman.

Steffe, L. P., \& Cobb, P. (1988). Construction of Arithmetical Meanings and Strategies. New York, NY: Springer New York. Retrieved from http://dx.doi.org/10.1007/978-1-4612-3844-7 
Steffe, L. P., \& Thompson, P. W. (2000). Teaching experiment methodology: Underlying principles and essential elements. Handbook of Research Design in Mathematics and Science Education, 267-306.

Stein, M. K., Remillard, J. T., \& Smith, M. S. (2007). How curriculum influences students learning. In Second handbook of research on mathematics teaching and learning (pp. 319-379). Greenwich, CT: Information Age Publishing.

Strauss, A. L., \& Corbin, J. M. (1998). Basics of qualitative research: techniques and procedures for developing grounded theory. Thousand Oaks: Sage Publications.

Thanheiser, E. (2009a). Preservice Elementary School Teachers' Conceptions of Multidigit Whole Numbers. Journal for Research in Mathematics Education, 40(3), 251-281.

Thanheiser, E. (2009b). Understanding base-ten numbers: Why don't you just tell them it is ten? In Proceedings of the thirty-first annual meeting of the North American Chapter of the International Group for the Psychology of Mathematics Education (Vol. 5, pp. 1292-1298). Retrieved from http://www.pmena.org/2009/proceedings/TEACHER\%20EDUCATION\%20PRESERVICE/tepBRR369749.pdf

Thanheiser, E. (2010). Investigating further preservice teachers' conceptions of multidigit whole numbers: refining a framework. Educational Studies in Mathematics, 75(3), 241-251.

Thanheiser, E. (2012). Understanding multidigit whole numbers: The role of knowledge components, connections, and context in understanding regrouping 3+-digit numbers. Journal of Mathematical Behavior, 31(2), 220-234.

Thanheiser, E. (2014). Developing preservice elementary teachers conceptions with welldesigned tasks: Explaining successes and analyzing conceptual difficulties. Journal of Mathematics Teacher Education, Submitted.

Thanheiser, E. (2015a). Developing prospective teachers' conceptions with well-designed tasks: explaining successes and analyzing conceptual difficulties. Journal of Mathematics Teacher Education, 1-32. http://doi.org/10.1007/s10857-014-9272-9

Thanheiser, E. (2015b). Leveraging historical number systems to build an understanding of the base 10 place value system. In Proceedings of the Twenty-third International Congress on Mathematical Education. Macau, China. 
Thanheiser, E., Browning, C., Edson, A. J., Lo, J.-J., Morton, C., Whitacre, I., \& Olanoff, D. (2014). Mathematical Content Knowledge for Teaching Elementary Mathematics: What do we know, what do we not know, and where do we go from here? Montana Mathematics Enthusiast - Special Issue., ((in preparation - special issue accepted)).

Thanheiser, E., Philipp, R. A., Fasteen, J., Strand, K., \& Mills, B. (2013). PreserviceTeacher Interviews: A Tool for Motivating Mathematics Learning. Mathematics Teacher Educator, 1(2), 137.

Thanheiser, E., \& Rhoads, K. E. (2009). Exploring preservice teachers' conceptions of numbers via the Mayan number system. In S. L. Swars, D. W. Stinson, \& S. Lemons-Smith (Eds.), Proceedings of the 31st annual meeting of the North American Chapter of the International Group for the Psychology of Mathematics Education. Atlanta, GA: Georgia State University. Retrieved from http://www.pmena.org/2009/proceedings/TEACHER\%20EDUCATION\%20PRESERVICE/tepBRR369758.pdf

The Design-Based Research Collective. (2003). Design-based research: An emerging paradigm for educational inquiry. Educational Researcher, 5-8.

Thompson, I. (2010). Issues in teaching numeracy in primary schools. Berkshire, England: McGraw-Hill, Open University Press.

Tirosh, D., \& Graeber, A. O. (1989). Preservice elementary teachers' explicit beliefs about multiplication and division. Educational Studies in Mathematics, 20(1), 7996.

Treffers, A. (1987a). Integrated column arithmetic according to progressive schematisation. Educational Studies in Mathematics, 18(2), 125-145.

Treffers, A. (1987b). Three dimensions: A model of goal and theory description in mathematics instruction-The Wiskobas Project. Dordrecht, Holland: Reidel Publishing Company.

Van Putten, C. M., Brom-Snijders, P. A. van den, \& Beishuizen, M. (2005). Progressive Mathematization of Long Division Strategies in Dutch Primary Schools. Journal for Research in Mathematics Education, 36(1), 44-73.

Verschaffel, L., Greer, B., \& De Corte, E. (2007). Whole number concepts and operations. In F. Lester (Ed.), Second handbook of research on mathematics 
Investigating Alternate Numeration Systems

teaching and learning (pp. 557-628). Charlotte, NC: Information Age Publishing. Retrieved from https://lirias.kuleuven.be/handle/123456789/153589

Vygotsky, L. S. (1962). Thought and Language. MIT Press.

Wawro, M., Rasmussen, C., Zandieh, M., Sweeney, G. F., \& Larson, C. (2012). An inquiry-oriented approach to span and linear independence: The case of the magic carpet ride sequence. PRIMUS, 22(8), 577-599.

Wawro, M., Sweeney, G. F., \& Rabin, J. M. (2011). Subspace in linear algebra: investigating students' concept images and interactions with the formal definition. Educational Studies in Mathematics, 78(1), 1-19.

Whitenack, J. W., \& Knipping, N. (2002). Argumentation, instructional design theory and students' mathematical learning: a case for coordinating interpretive lenses. The Journal of Mathematical Behavior, 21(4), 441-457.

Yackel, E., Underwood, D., \& Elias, N. (2007). Mathematical Tasks Designed to Foster a Reconceptualized View of Early Arithmetic. Journal of Mathematics Teacher Education, 10(4-6), 351-367.

Zandieh, M., \& Rasmussen, C. (2010). Defining as a mathematical activity: A framework for characterizing progress from informal to more formal ways of reasoning. The Journal of Mathematical Behavior, 29(2), 57-75.

Zaslavsky, O., \& Peled, I. (1996). Inhibiting Factors in Generating Examples by Mathematics Teachers and Student Teachers: The Case of Binary Operation. Journal for Research in Mathematics Education, 27(1), 67-78. http://doi.org/10.2307/749198

Zazkis, R. (1999). Challenging basic assumptions: mathematical experiences for preservice teachers. International Journal of Mathematical Education in Science and Technology, 30(5), 631-650.

Zazkis, R., \& Khoury, H. A. (1993). Place Value and Rational Number Representations: Problem Solving in the Unfamiliar Domain of Non-decimals. Focus on Learning Problems in Mathematics, 15(1), 38-51.

Zazkis, R., \& Khoury, H. A. (1994). To the right of the decimal point: Preservice teachers' concepts of place value and multidigit structures. Research in Collegiate Mathematics Education, 1, 195-224. 
Investigating Alternate Numeration Systems

\section{Appendix A: Codes for Textbook Analysis}

Table 1. Final codes used in the textbook coding process.

\begin{tabular}{|c|c|c|c|}
\hline \multicolumn{4}{|c|}{ Types of investigations and exercises } \\
\hline \multicolumn{2}{|c|}{$\begin{array}{l}\text { Comparing between systems: } \\
\text { Translate OUT OF base ten, } \\
\text { Translate TO base ten, } \\
\text { Comparing number systems, } \\
\text { Connecting to a standard algorithm } \\
\text { Looking for patterns between systems, } \\
\text { Describing advantages or disadvantages, } \\
\text { General statements about types of number } \\
\text { systems } \\
\text { Other: } \\
\text { Decimals or fractions, Non-routine / other }\end{array}$} & \multicolumn{2}{|c|}{$\begin{array}{l}\text { Immersion within a system: } \\
\text { Within base operations, } \\
\text { Within base up or down by one, } \\
\text { Looking for patterns within a system, } \\
\text { PST investigates an unexplained system, } \\
\text { PSTs invent a system, } \\
\text { PSTs invent an algorithm / procedure, } \\
\text { Reflecting on learning a number system } \\
\text { Operation Table }\end{array}$} \\
\hline \multicolumn{4}{|c|}{ Rationales for studying other number systems } \\
\hline \multicolumn{2}{|c|}{$\begin{array}{l}\text { Mathematical structure } \\
\text { Appreciate difficulty or power of base ten, } \\
\text { To make sense of mathematical structure, } \\
\text { Deepen understanding of base ten, } \\
\text { Insight into algorithms } \\
\text { Other: You may want/have to teach } \\
\text { alternate bases, Other bases are used in } \\
\text { the world }\end{array}$} & \multicolumn{2}{|c|}{$\begin{array}{l}\text { Children's thinking: } \\
\text { Experience what children experience } \\
\text { Focus on specific challenges for children } \\
\text { Understand children will struggle } \\
\text { (general) }\end{array}$} \\
\hline \multicolumn{4}{|c|}{ Which alternate systems } \\
\hline $\begin{array}{l}\text { Historic Systems Codes: } \\
\text { Egyptian, } \\
\text { Roman, } \\
\text { Babylonian, } \\
\text { Mayan, } \\
\text { Hindu-Arabic, } \\
\text { Historic-other. }\end{array}$ & \multicolumn{2}{|c|}{$\begin{array}{l}\text { Modern Number Systems } \\
\text { Codes: } \\
\text { Base five, } \\
\text { Binary, } \\
\text { Time base 60, } \\
\text { Modern alternate system. }\end{array}$} & $\begin{array}{l}\text { Other related co } \\
\text { Cultural Facts, } \\
\text { Grouping conte. } \\
\text { problem, } \\
\text { Language conve } \\
\text { alternate bases }\end{array}$ \\
\hline Prompts within the narrative & \multicolumn{2}{|c|}{ Visual Models } & \\
\hline $\begin{array}{l}\text { For inquiry - PST does work } \\
\text { For inquiry - solution provided } \\
\text { For practice - PST does work } \\
\text { For practice - solution } \\
\text { provided }\end{array}$ & \multicolumn{2}{|c|}{$\begin{array}{l}\text { Abacus - alternate } \\
\text { base, } \\
\text { Base pieces (Dienes), } \\
\text { Bundles of sticks }\end{array}$} & $\begin{array}{l}\text { Humans invent math, } \\
\text { Properties of operations, } \\
\text { Zero as a focus, } \\
\text { How children learn about } \\
\text { number }\end{array}$ \\
\hline
\end{tabular}


Investigating Alternate Numeration Systems

\section{Appendix B: Multiplication Task Sequence}

For each of the four primary tasks in the task sequence, I discuss the task in three distinct parts. The first part outlines the goals of the task, the second part includes the actual task and the rationale for the task design using RME design heuristics, and the third part outlines the student mathematical activity anticipated from engagement with the task.

The sequence of tasks was intended to promote student mathematical activity around two key ideas of multiplication, with the first key idea answering the question "what is multiplication" and the second key idea answering the question "what are efficient strategies to perform multiplication." I anticipated that repeated addition and area would emerge as models of what multiplication means and as useful models for developing sophisticated strategies for multidigit multiplication. Efficient and sophisticated strategies for multidigit multiplication involve splitting numbers apart using the distributive property, creating a partial products model for performing multiplication. The emergence of this partial products model was the primary focus of the fourth task in the sequence, which involved the creation and justification of a general strategy for multidigit multiplication in base five.

\section{Task 1: Focus on Repeated Addition}

Mathematical Goals of Task 1. The mathematical goals of Task 1 focused on bringing forth an initial model of multiplication as repeated addition. The three primary (and somewhat overlapping) mathematical goals were for PSTs to:

1. Develop a repeated addition model of multiplication and name this model. 
Investigating Alternate Numeration Systems

2. Give meaning to the multiplier and multiplicand in a multiplication problem. In the problem $a \times b$, the first number ( $a$ ) refers to the number of groups and is called the multiplier. The second number $(b)$ refers to the size of each group and is called the multiplicand.

3. Generate an initial definition of multiplication.

\section{Task 1:}

Find the product $3_{\text {five }} \times 23_{\text {five }}$ and explain your solution strategy.

Follow-up: Create a definition of multiplication that would be appropriate to use with a second grader.

Figure 1: Task 1 is intended to bring forth the repeated addition model for multiplication

Rationale for the Design of Task 1. The introductory task was designed using the guided reinvention heuristic from RME (Gravemeijer, 1998). Repeated addition is a primitive model for multiplication (Fischbein et al., 1985) and it is the model that PSTs tend to rely on (Graeber, 1999; Tirosh \& Graeber, 1989). Beginning with repeated addition allows PSTs to engage with the task using their own informal strategies. The more sophisticated area/array model can be built upon repeated addition, or, if built separately, can be connected to the repeated addition model. This connection was examined in the section after Task 2 (see Figure 9). With this trajectory in mind, the series of tasks shifted from ones that can be easily represented with a repeated addition model to ones that beg for a more sophisticated solution strategy.

The introductory task of $3_{\text {five }} \times 23_{\text {five }}$ used a small, single digit multiplier of 3 and a two digit multiplier of $23_{\text {five }}$. While elementary school curricula may start with single digit by single digit problems, I anticipated that a two digit multiplicand would not be problematic to the PSTs, who have had prior experience building multidigit numbers in 
base five. The small, single digit multiplier of three was selected to make the repeated addition strategy a feasible and natural option for PSTs. Having previously engaged in addition in base five, the task of tripling a base five number could be built upon previously established work with addition. A previous pilot of the tasks revealed a struggle with multidigit multipliers, as PSTs focused on the separate digits of the multiplier, rather than the quantity represented by the multiplier (Fasteen et al., 2015). The task sequence did not attempt to avoid this struggle, but to postpone it until Task 2, after students had the opportunity to begin to establish the repeated addition model in Task 1. This struggle is discussed further in the rationale for Task 2.

In addition to the mathematical goals of Task 1, the task also served as an opportunity to gather evidence of PSTs regrouping skills and initial strategies for multiplication. As the students had already engaged with base five addition and subtraction, I anticipated that regrouping in base five would not be problematic. Further discussion of anticipated student strategies takes place in the following section.

Anticipated Student Mathematical Activity for Task 1. Based on my past experience using this task with preservice teachers, I anticipated two primary tactics for solving the task $3_{\text {five }} \times 23_{\text {five. }}$. The first strategy relies upon repeated addition and the second strategy relies on modifying the standard U.S. algorithm for multidigit multiplication.

The repeated addition strategy tends to take two formats. The first involves creating 3 sets of the manipulatives corresponding to $23_{\text {five. }}$. An image of this is provided in Figure 2a. The manipulatives are then rearranged to group like objects together (Figure $2 b$ ). If there are 5 or more of one manipulative, they are regrouped to the next 
larger manipulative, i.e. 5 longs are regrouped into a flat (Figure 2 2c). The final solution is represented as 1 flat, 2 longs, and 4 ones (Figure 2d).

\begin{tabular}{|c|c|c|c|}
\hline 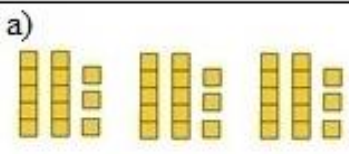 & 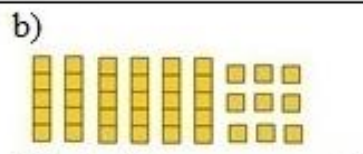 & 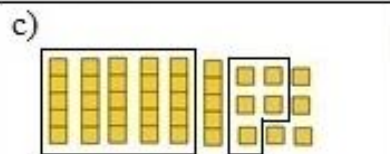 & 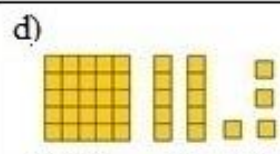 \\
\hline $\begin{array}{l}3_{\text {five }} \times 23_{\text {five }} \text { is modeled } \\
\text { as } 3 \text { groups of } 23_{\text {five }}\end{array}$ & $\begin{array}{l}\text { Manipulatives rearranged } \\
\text { to place longs with longs } \\
\text { and ones with ones }\end{array}$ & $\begin{array}{l}5 \text { longs regroups as a flat } \\
5 \text { ones regrouped as a long }\end{array}$ & $\begin{array}{l}\text { Solution of } 1 \text { flat } 2 \\
\text { longs } 4 \text { ones is } \\
\text { written } 124_{\text {five }}\end{array}$ \\
\hline
\end{tabular}

Figure 2: A solution to $3_{\text {five }} \times 23_{\text {five }}$, modeled with manipulatives

Alternately, the repeated addition strategy can be executed symbolically by representing $3_{\text {five }} \times 23_{\text {five }}$ as $23_{\text {five }}+23_{\text {five }}+23_{\text {five }}$ and relying on prior knowledge about addition strategies. In the case of doing symbolic repeated addition, students tend to stack the three addends vertically, as in Figure 3. Then the three copies of 3 are summed to arrive at 9 , which is regrouped as 1 long and 4 units. Then the 3 copies of 2 longs are summed to arrive at 6 longs, plus one more long from the prior step. These 7 longs are regrouped as 1 flat 2 longs. It is possible for PSTs to execute this strategy without recognizing that the three $2 \mathrm{~s}$ refer to longs and that the regrouped 7 longs become 1 flat and 2 longs. Prior research indicates that PSTs may not explicitly focus on the type of unit being summed and regrouped (Thanheiser, 2009a).

\begin{tabular}{|l|l|l|l|}
\hline & 1 & 1 & 1 \\
23 & 23 & 23 & 23 \\
23 & 23 & 23 & 23 \\
+23 & $\underline{+23}$ & $\frac{+23}{74}$ & $\frac{+23}{124}$ \\
\hline 9 & 4 & five \\
\hline Step 1: Three 3s & Step 2: Nine ones & Step 3: Three 2s & Step 4: The 7 is \\
are summed to 9. & are regrouped to 1 & plus 1 extra are & regrouped as 1 flat \\
& long 4 ones & summed to 7. & 2 longs. \\
\hline
\end{tabular}

Figure 3: Steps for adding 3 copies of $23_{\text {five }}$ using symbols. 
The repeated addition strategy, either symbolically or physically represented, indicates an appropriate mental model of multiplication. The other anticipated student strategy is to modify the standard U.S. algorithm for multidigit multiplication. This strategy does not clearly indicate what type of mental model, if any, the PST holds for multiplication. In order to modify the standard algorithm for multiplication to the base five context, the students begin by writing the problem vertically, with $23_{\text {five }}$ on the top and $3_{\text {five }}$ on the bottom (Figure 4a). Then 3 is multiplied by 3 and the result of 9 is split into 1 long and 4 ones, with the 4 recorded below the 3 and the 1 recorded above the 2 (Figure $4 \mathrm{~b}$ ). In the next step, 3 is multiplied by the 2 in $23_{\text {five }}$ to reach 6 . The one from the prior step is added to the 6 to arrive at 7, which is regrouped as 1 flat and 2 longs (Figure 4c). The digits "12" are recorded below the prior value of 4 , but shifted to the left one spot. Some PSTs place a 0 after the " 12 " to indicate the place value, while others leave the spot blank or place some other symbol in this location. Then the partial products of 4 and 120 are summed to arrive at $124_{\text {five }}$.

\begin{tabular}{|c|c|c|}
\hline $\begin{array}{r}23 \\
\times \quad 3 \\
\end{array}$ & $\begin{array}{r}1 \\
23 \\
\times \quad 3 \\
4\end{array}$ & $\begin{array}{c}1 \\
23 \\
\times 3 \\
4 \\
\underline{12} 0 \\
124_{\text {five }}\end{array}$ \\
\hline $\begin{array}{l}\text { a) The } 23 \text { is written } \\
\text { above the } 3 \text {, with the } \\
\text { number aligned by place } \\
\text { value (ones above ones) }\end{array}$ & $\begin{array}{l}\text { b) } 3 \text { is multiplied by } 3 \\
\text { and the product of } 9 \text { is } \\
\text { regroup to } 1 \text { long } 4 \text { units }\end{array}$ & $\begin{array}{l}\text { c) } 3 \text { is multiplied by } 2 \text { to } \\
\text { get } 6 \text {, plus the extra } 1 \\
\text { from the prior step. This } \\
7 \text { is regrouped as } 12 \text { in } \\
\text { base five. }\end{array}$ \\
\hline
\end{tabular}

Figure 4: The standard U.S. algorithm for multiplication, modified for the base five context. 
Investigating Alternate Numeration Systems

The use of the standard algorithm for multiplication creates a dilemma for me as the instructional designer. I have two sometimes conflicting goals: building on PSTs' prior knowledge and helping PSTs to build conceptually based understanding of whole number concepts. These goals conflict when PSTs prior knowledge is procedurally based. To mediate these conflicting goals, I can encourage students who choose to use the algorithm to create a visual model to unpack the algorithm. The algorithm can be viewed as a shorthand version of a sense-making process, but only if the students first make sense of how to model multidigit multiplication. Within the teaching experiments I was able to build a small-group culture which focused on sense making and explanations, which helped to direct students' attention to valuing the "why" behind procedures.

\section{Task 2: Multiplication by the Base $\left(1_{\text {five }}\right)$}

Mathematical Goals of Task 2. While the first task in the sequence was intended to promote the repeated addition model of multiplication, the second task was intended to (1) expand the use of the repeated addition model to multidigit multipliers and to (2) bring a focus to a structurally important question: "What happens when you multiply by $10_{\text {five? }}$ ?" These two primary goals are expanded upon below.

1. Make sense of a two digit multiplier in base five.

a) Make sense of the multiplier as a quantity rather than concatenated single digits that are dealt with separately.

b) Use the multiplier to determine "how many copies" are needed of the multiplicand.

2. Discover and make sense of the Times Base Rule 
Investigating Alternate Numeration Systems

a) Recognize that multiplying a specific number by $10_{\text {five }}$ means creating 5 copies of that number.

b) Recognize that 5 copies of a unit type can be regrouped to one copy of the next larger unit type.

c) Articulate and justify a general statement about multiplication by $10_{\text {five }}$ which highlights the idea of shifting all digits to the left one slot.

Rationale for the Design of Task 2. The guided reinvention design heuristic suggests that the instructional designer should be aware of and incorporate key landmarks in the path of reinventing (Gravemeijer, 1998). Based on student generated strategies (Ambrose et al., 2003) and as well as an analysis of the standard U.S. algorithm for multiplication (see Figure 2-7). One key landmark in creating efficient multiplication algorithms involves determining efficient ways to split up numbers. Multiplication by $10_{\text {five }}$ is particularly efficient and I hypothesized that it would play a central role in PST generated strategies in base five. Therefore, Task 2 focused explicitly on making sense of multiplication by $10_{\text {five. }}$.

Task 2:

Find the product $10_{\text {five }} \times 23_{\text {five }}$ and explain your reasoning. Connect your explanation to a visual model.

Follow-ups:

- Make a conjecture about multiplying by $10_{\text {five. }}$ Justify your conjecture.

- Comparison across bases: 10 × $23=230$ and $10_{\text {five }}$ x $23_{\text {five }}=230_{\text {five. }}$ Is this a coincidence or something more?

Figure 5: Task 2 focused on making sense of multiplication by the base $\left(10_{\text {five }}\right)$

In a pilot of the instructional sequence, students struggled to make sense of a multidigit base five multiplier in a two digit by two digit problem. When the multiplier 
was a single digit base five number, the students were able to act as if it were a base ten multiplier. That is, $2_{\text {five }}=2_{\text {ten }}, 3_{\text {five }}=3_{\text {ten }}$, and $4_{\text {five }}=4_{\text {ten. }}$. Because the multiplier was small and familiar, the repeated addition model appeared naturally. When attempting a two digit multiplier, such as $12_{\text {five }}$, students tended to either (a) muddle base five with base ten and treat $12_{\text {five }}$ as twelve (in base ten) rather than as 1 five and 2 units (equivalent to 7 in base ten), or (b) use a modified version of the standard U.S. algorithm for multiplication which they struggled to justify. In order to make sense of the two digit multiplier, the PSTs must develop a strategy to coordinate the two digits in base five. Task 2 provided an opportunity to investigate a foundational example, when the multiplier is $10_{\text {five. }}$.

The selection of the numbers for Task 2 was done with the purposeful intention of guiding student mathematical activity along a path that is grounded in prior experiences and lays a foundation for more sophisticated strategies in later tasks. I chose to continue to use the multiplicand of $23_{\text {five }}$ so that this problem could be seen by PSTs as building up from the prior problem of $3 \times 23_{\text {five. }}$. I selected the multiplier of $10_{\text {five }}$ for a number of reasons. First, I was aware of students' tendency to confound $10_{\text {five }}$ (worth five) with the base ten number 10 (ten), referring to both $10_{\text {five }}$ and 10 as "ten" and modeling $10_{\text {five }}$ inconsistently as five or ten groups. I wanted students to confront this confusion directly. Second, because $10_{\text {five }}$ is a relatively small number, the repeated addition model continues to be an efficient solution strategy. My third reason for selecting $10_{\text {five }}$ as the multiplier is that in base five, multiplying by the value of the base $\left(10_{\text {five }}\right)$ has the effect of shifting all of the digits in a number to the left one slot. This feature (a) focuses attention of the 
multiplicative structure of base five and (b) becomes a building block for later multiplication strategies.

Prior research has shown that PSTs may struggle to make sense of what happens when a zero is appended to a number in an alternate base (Thanheiser, 2014a; Thanheiser \& Rhoads, 2009). Task 2 involves an exploration of a phenomenon that causes a zero to be appended, namely, multiplying by $10_{\text {five }}$. The first follow-up question is intended to invite PSTs to make the conjecture that $10_{\text {five }}$ of one base five unit (such as a one, long, or flat) can be regrouped to create one of the next larger item. For example, $10_{\text {five }}$ ones can be regrouped to a long and $10_{\text {five }}$ longs can be regrouped to a flat, and so on. An image of this regrouping can be found in Figure 6 .

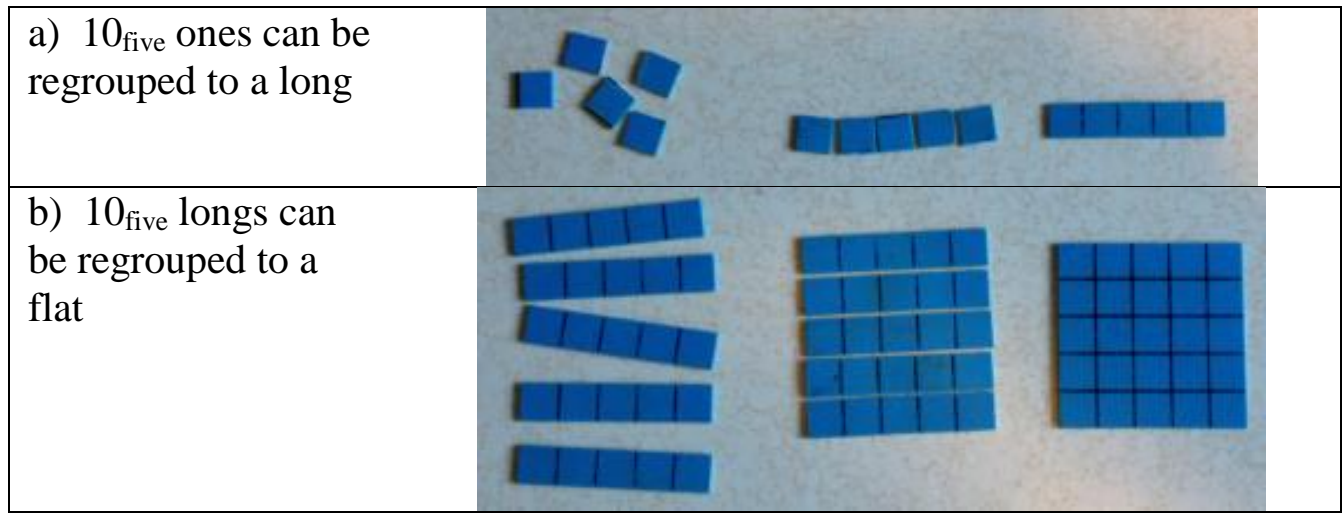

Figure 6: $10_{\text {five }}$ copies of one unit can be regrouped into the next larger unit.

The second follow-up question was intended to leverage PST curiosity about why the problems $10 \times 23=230$ and $10_{\text {five }} \times 23_{\text {five }}=230_{\text {five }}$ look similar, despite the differences in bases (See Figure 7). The prompt was intended to encourage PSTs to step back from base five and make a conjecture across bases. By examining the role of multiplying by the number that looks like 10 in each base (notated as 10 in base ten, $10_{\text {five }}$ in base five, or $10_{\text {base }}$ in a generic base), PSTs would focus on the multiplicative structure 
of place value systems. That is, PSTs would focus on how each column is worth "base" times more than the column to its right, where the "base" depends on the number system.

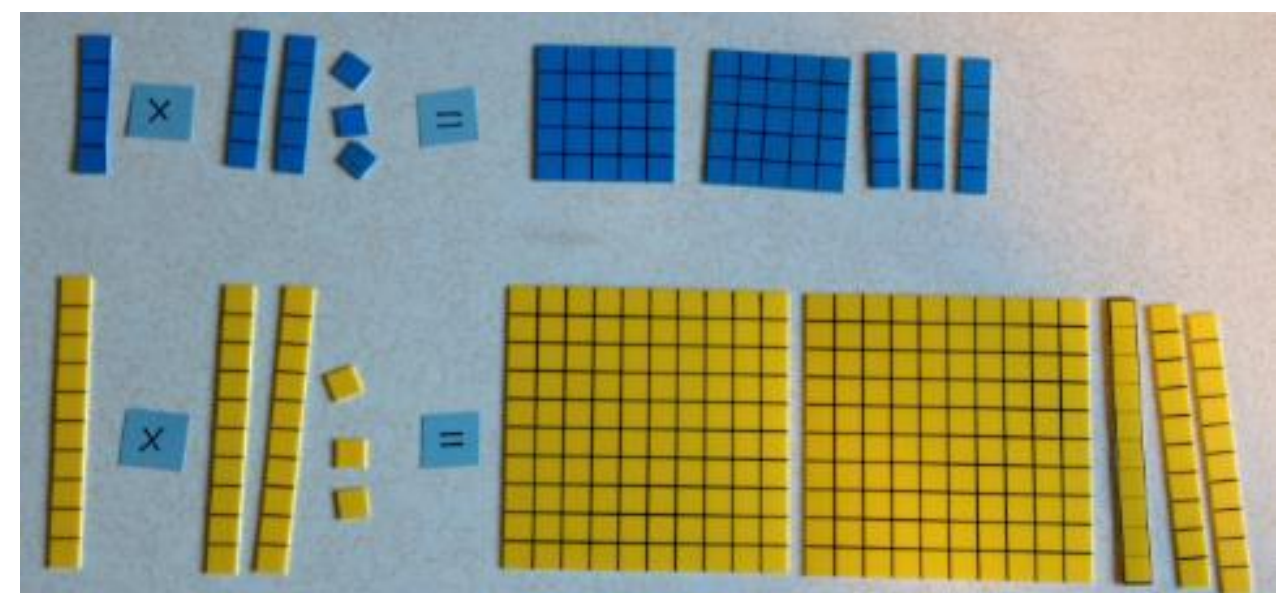

Figure 7: The similarities between $10 \times 23=230$ in base ten and $10_{\text {five }} \times 23_{\text {five }}=230_{\text {five }}$ focus attention on structure of place value systems.

Multiplication by $10_{\text {five }}$ as a Stepping Block. The multiplication by $10_{\text {five }}$ task was designed to serve two critical purposes. The first purpose was to provide a context that encouraged making sense of the multiplicative structure of base five. This purpose is captured in the explicit goals listed above. The second purpose ${ }^{19}$ of Task 2 was to develop a building block for more advanced strategies for multidigit multiplication. These strategies rely upon breaking numbers along place value and the use of the distributive property to build a partial products model of multiplication.

To get a flavor for these strategies, consider the example of $43 \times 24$ in base ten. The 43 can be split into 40 and 3 . In order to multiply 40 by 24 , you can first multiply 4 by 24 (96) and then note that 40 is ten times more than 4 , so $40 \times 24$ should be ten times more than $4 \times 24$, resulting in a partial product of 960 . Then add 3 more copies of 24 (72) to arrive at the final solution of 1032. This strategy is efficient because making

\footnotetext{
${ }^{19}$ This second purpose is not listed as one of the mathematical goals for Task 2 because it will not surface for PSTs until Tasks 3 and 4.
} 
something ten times larger is an efficient task in base ten. It is this efficiency that underlies the standard U.S. algorithm for multiplication as well as student generated solution strategies.

Anticipated Student Mathematical Activity for Task 2. I expected that PSTs' initial strategies for Task 2 would be similar to their strategies for Task 1. PSTs could rely on repeated addition, either with symbols or images/manipulatives, or PSTs could use a modified standard algorithm with an explicit goal of connecting the algorithm to a visual model. I anticipated that by focusing the conversation on linking the visual models to the multiplication concept, the pursuit of a sense making solution could begin to remove the standard algorithm from its mathematical pedestal as the unquestioned best strategy.

As indicated in the rationale, Task 2 is more difficult than Task 1 because of the two digit multiplier. PSTs must unpack the meaning of $10_{\text {five }}$ groups of $23_{\text {five }}$ by first determining how much $10_{\text {five }}$ is. In a pilot of the study, the PSTs struggled with the value of $10_{\text {five }}$ and confused the base ten number 10 (ten) with the base five number $10_{\text {five }}$ (worth five). I anticipated that the PSTs would draw on the base five manipulatives to help them to find meaning for $10_{\text {five. }}$ In my pilot study, one group of PSTs used the manipulative corresponding to $10_{\text {five }}$ (a long) and for each unit within the long, the PSTs gathered one copy of the group of manipulatives representing $23_{\text {five }}$ (Fasteen et al., 2015). The PSTs, who had previously struggled with the value of $10_{\text {five }}$, stressed the usefulness of touching each unit in the long while explicitly linking each unit to a group of size $23_{\text {five }}$ (Figure 8). This allowed the PSTs to find an explicit role for each of the numbers in the problem $10_{\text {five }}$ x $23_{\text {five. }}$. 


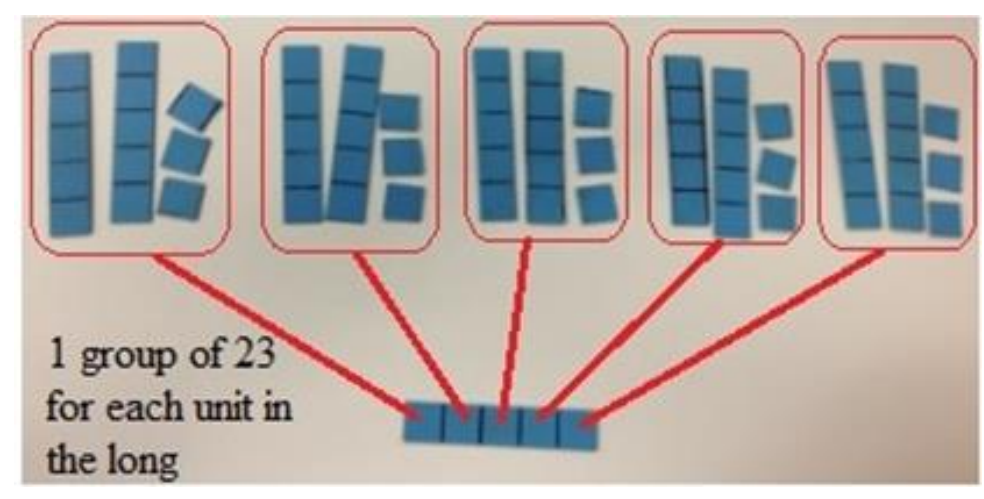

Figure 8: Student strategy for $10_{\text {five }}$ x $23_{\text {five }}$ involves creating a collection of $23_{\text {five }}$ for each unit in the long $\left(10_{\text {five }}\right)$.

After determining how to solve the specific problem $10_{\text {five }} \times 23_{\text {five }}$, there is a shift to focusing on a general statement about multiplying by the base $\left(10_{\text {five }}\right)$. This shift could be the result of their own curiosity or teacher press about the idea I refer to as the Times Base Rule. The Times Base Rule answers the question "Does multiplying a number by $10_{\text {five }}$ always result in appending a zero to the number being multiplied?" This question could be asked in any base, with the value " $10_{\text {five }}$ " replaced by the value of the base $\left(10_{\text {base }}\right)$ for that number system. In base ten, students may refer to this idea as the "Times Ten Rule.”

I anticipated that students would form a conjecture that when multiplying a number by $10_{\text {five }}$ a zero is appended to the number. This conjecture could come from trying one or several examples, from making an analogy with the Times Ten Rule in base ten, or from making an argument about the modified standard algorithm. This hypothesis comes from a pilot of the task (Fasteen et al., 2015). To move from conjecture to justification, PSTs need to address the question of "why" a zero is appended. To make sense of this, PSTs would combine two key facts: multiplying a number by $10_{\text {five }}$ results in 5 copies of that number and five copies of one object in base five results in one object 
of the next larger type. An example of this sense-making approach can be found in Fasteen, et al. (2015).

Linking the Repeated Addition Model to Area. Tasks 1 and 2 relied upon the repeated addition model of multiplication. The next two tasks shift focus to the area/array model of multiplication. The repeated addition model can be linked to the area/array model by rearranging the imagery of a repeated addition problem. Consider the example of $10_{\text {five }} \times 23_{\text {five }}$, which can be illustrated as 5 piles of size $23_{\text {five. }}$. By shifting the 5 piles of size $23_{\text {five }}$ (Figure 9 a) to 5 rows of size $23_{\text {five, }}$, the repeated addition model begins to take the shape of a rectangle or an array (Figure 9b). Slide the 5 rows together, and a rectangle with dimensions $10_{\text {five }}$ (or 5) by $23_{\text {five }}$ is created (Figure 9c). It is possible that PSTs would reinvent this transition to area within the $10_{\text {five }} \times 23_{\text {five }}$ task, but pilot data indicates that this is unlikely to happen spontaneously. PSTs tend to rely on the primitive model of repeated addition (Graeber et al., 1989; Tirosh \& Graeber, 1989).

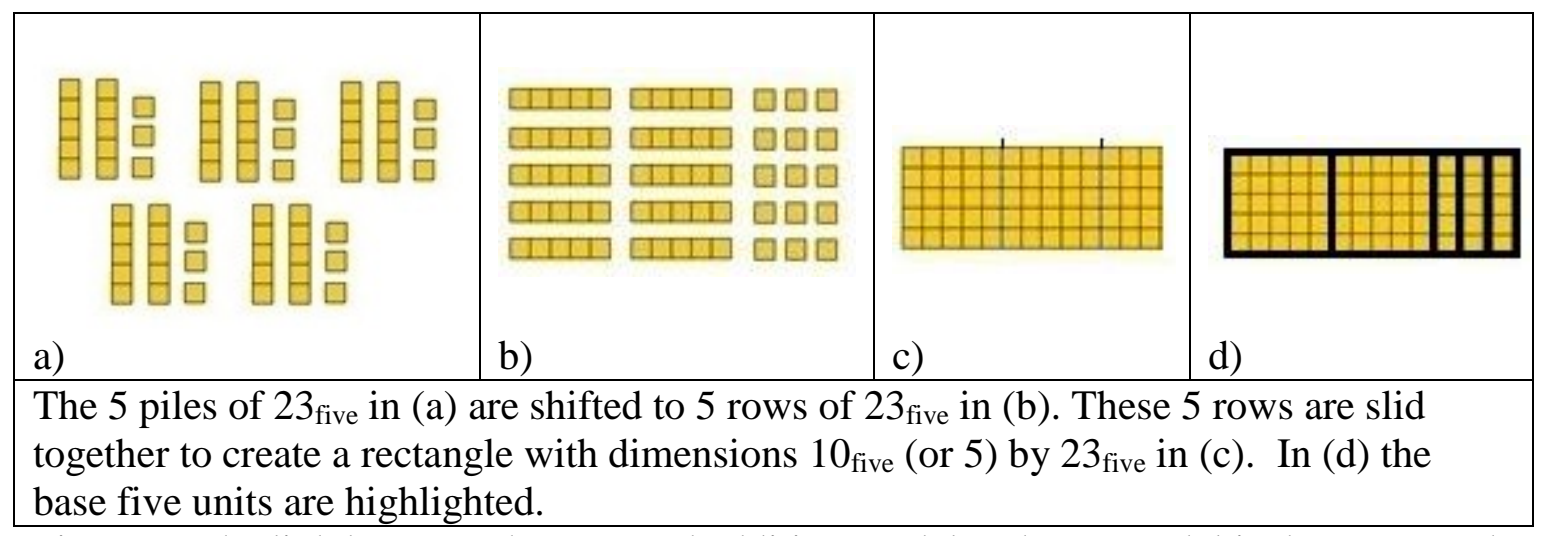

Figure 9: The link between the repeated addition model and area model is demonstrated visually.

After the emergence of the repeated addition model of multiplication with the first two tasks, the following tasks were intended to shift the focus from repeated addition to the area model. In a pilot of the instructional sequence, students were given a two digit 
by two digit numeric problem ( $42_{\text {five }} \times 31_{\text {five }}$ ). The area model did not emerge naturally for many of the groups. Some groups continued to rely on the repeated addition model while others used a modified version of the standard algorithm from base ten. To encourage the emergence of the area model as a model of multiplication, Task 3 was altered to provide an area context rather than a symbolic problem. The search for and study of a particular phenomenon to promote the emergence of a key model is referred to as didactical phenomenology (Gravemeijer, 1998). I discuss the phenomenological analysis of the area context further in the context of Tasks 3 and 4 , along with a discussion of the partial products strategy for multiplication.

\section{Task 3: Introducing Area}

Mathematical Goals of Task 3. The primary goal of Task 3 was to promote the emergence of an area model for multiplication, which would play a central role in the development of a general multiplication strategy in Task 4. Specific sub-goals are listed below.

- Develop the area model of multiplication and link this model with the prior repeated addition model of multiplication.

- Build a situated solution strategy for a specific multidigit multiplication problem (this becomes an example of a type of problem in Task 4: The Box of Rectangles)

- Draw explicit attention to PSTs' implicit use of the distributive property and begin to unpack the role of the distributive property in PST generated solution strategies. Note: I intended to help them to identify and apply a name to a property they are using, rather than introduce a property that is seen as external and teacher provided. 
Task 3:

\section{Measuring a rectangle in base five}

Each table will receive one rectangle. Your goal is to examine the size of the rectangle in base five. Determine the length, width, and area of the rectangle and discuss how the three quantities (length, width, and area) are related. Record two or more strategies for calculating the area and explain why those strategies make sense. Be sure to connect your visual models with written solutions.

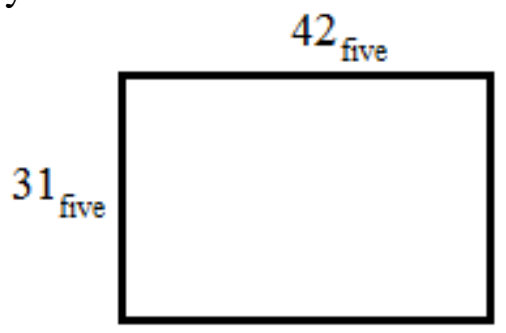

Follow-up questions:

- In the first two base five problems, we used the repeated addition model for multiplication. How is your approach for Task 3 similar to the repeated addition model and how is it different than the repeated addition model?

- [Discuss the distributive property, using one of the student generated strategies to discuss how the area was split apart.]

I noticed that in the strategy used by group , the rectangle was split up into smaller pieces and the area of each piece was found separately (i.e. it was multiplied separately).

$\circ$ Why is that allowed?

$\circ$ Why is that useful?

Is that always an option?

o What is it called?

Figure 10: Task 3 provides a rectangle context, intended to lead to the emergence of an area model of multiplication.

Rationale for the design of Task 3. In a prior pilot of the study, the task

following $10_{\text {five }} \times 23_{\text {five }}$ was $42_{\text {five }} \times 31_{\text {five. }}$. One mathematical goal of this task was for some small group to discover the area model as a useful tool to both solve the problem and justify the solution strategy. If some small group within the whole class discovered this area strategy, then the strategy could be shared with the whole class. As fragile as this approach is within a full class teaching experiment, is becomes unreasonable within a smaller scale teaching experiment with a limited number of students. Returning to the 
Investigating Alternate Numeration Systems

design heuristics of RME, particularly didactic phenomenology, it became clear that providing the realistic context of a rectangular area task has the potential to introduce a phenomenon (rectangular area) which begs for mathematizing (Gravemeijer, 1998). The guided reinvention heuristic does not require that the students must discover or invent the rectangular area context on their own. The instructional designer should select appropriate contexts to encourage investigation of worthwhile phenomena.

Didactic phenomenology for rectangular area context. The design heuristic of didactic phenomenology invites task designers to analyze potential contexts and tasks in light of how those tasks will encourage mathematical activity that leads to the development of key mathematical models. An analysis of the rectangle context for multiplication reveals several useful features of this context. First, the rectangle model provides a visual model for multidigit multiplication that expands in an efficient manner to larger numbers. For example, it is possible to sketch a rectangle with dimensions $31 \mathrm{x}$ 42 much more quickly than sketching 31 collections of size 42 . The rectangle image can be partitioned into (useful) sub-rectangles so that one can find the area of each subrectangle and sum these sub-areas to find the area of the full rectangle. The strategic subdividing of the rectangle becomes the basis for a partial products model for multiplication. Initially the partial products model can be seen as a model of PSTs strategies, which the PSTs may not be explicitly aware of. This partial products model is based on the distributive property of multiplication over addition, which indicates that if one or both factors in a multiplication problem are split into an addition problem, then each addend can be multiplied by the other factor and summed without altering the total. A base ten example is provided in Figure 11. 


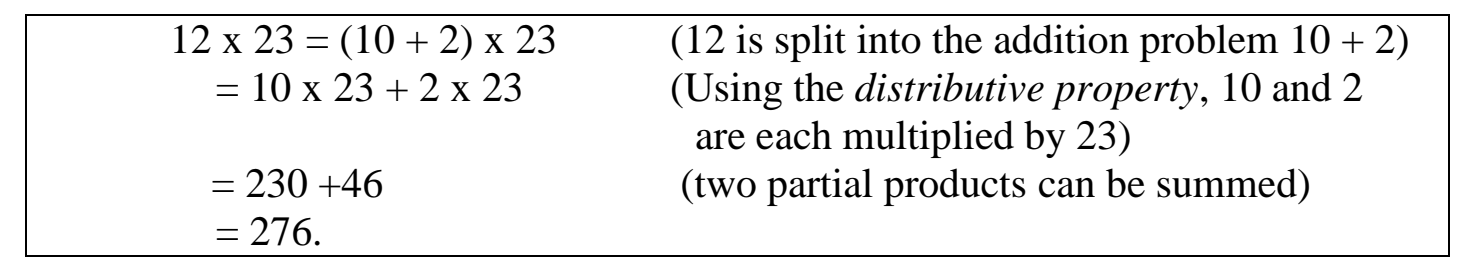

Figure 11: An example of the role of the distributive property in multiplying $12 \times 23$ in base ten.

Further analysis of the rectangular area context for multiplication reveals its utility beyond whole number multiplication tasks. The visual model of area is appropriate for multiplication of fractions, decimals, polynomials, and functions (see Figure 2-13). The strategy of subdividing areas and working with simpler subareas continues to be an efficient and conceptually sound tool for these more advanced number domains.

Rationale for Task 3 Follow-up Questions. A primary goal of Task 3 was for PSTs to develop a situated model or strategy for s specific multiplication problem so that Task 3 could be referred to as an example of the type of problems explored in Task 4. Additional goals about connecting models for multiplication and making sense of the distributive property were a focus of the follow-up questions for Task 3.

The first follow-up question to Task 3 was intended to make explicit a connection between the two mathematical models of multiplication: area and repeated addition. It is not enough to build two models for multiplication. Prospective teachers must be aware of how those two models are linked and PSTs should be able to describe advantages of each model. For example, the repeated addition model builds on children's prior work with addition, making multiplication a natural extension of addition. On the other hand, 
Investigating Alternate Numeration Systems

the area model embodies an efficiency that is lacking in the repeated addition model. The area model expands to the number domains of fractions, decimals, and algebraic expressions, while the repeated addition model becomes problematic outside of whole numbers. Imagine, for example, finding $3 / 4$ groups of size $1 \frac{1 / 2}{2}$ or $(x+2)$ groups of size $(-3 y)$.

The second follow-up question focused on the role of the distributive property in student strategies. The distributive property is embedded in most student invented strategies (at least those that move past direct modeling or simple repeated addition). By calling attention to the distributive property as it is already being used in practice, the ownership of the property goes to the students rather than an external source such as a teacher or textbook.

Anticipated Mathematical Activity for Task 3. For the first part of the area task, students would engage in the act of measuring the rectangle provided. I anticipated that students would place longs and units along each dimension to determine the value, in base five, of the side lengths. Other options include measuring entirely in units (rather than longs and units) or measuring the rectangle in inches or centimeters, determining the length of a unit square, and dividing to find the number of units. The latter options could involve having a temporary answer in base ten before shifting to base five.

Once the students have found the linear dimensions of the rectangle, I anticipated that they would fill the rectangle with base five manipulatives in order to find the area. While it is possible that PSTs would fill the rectangle with ones or fill it haphazardly with a variety of base five pieces, prior experience led me to believe they would fill the rectangle systematically, with larger pieces placed first and small pieces filling the extra 
space. After filling the shape, PSTs would then regroup the manipulatives to create a minimal collection to represent the quantity associated with the area. At this point the PSTs could be tempted to declare that the problem has been solved and move to the next task. At this point, however, they would have only a procedural solution strategy based on concrete manipulations and this strategy would not link the linear dimensions to the area, beyond the perfunctory statements that length times width equals area.

The prompt for this task asks for two or more strategies and an explanation of why those strategies make sense. To encourage multiple strategies I could provide recording papers labeled "strategy 1" and "strategy 2." Possible secondary strategies include:

- Repeated addition. The inefficiency of this strategy/model for multiplication of larger numbers may serve to highlight the benefits of strategic use of the area model. PSTs may increase the efficiency of the repeated addition model by grouping quantities together, such as $10_{\text {five }}$ copies of a number.

- Standard algorithm, modified to base five. PSTs who use the standard algorithm may struggle to justify which place value location to record single digit calculations in. For example, when multiplying 3 longs by 4 longs, PSTs may be unaware that the result, prior to regrouping, will be a quantity of flats. Connecting the standard algorithm to the area image may help PSTs to generate a reasonable justification for this issue. Because the standard algorithm focuses on single digit by single digit multiplication, this strategy is sometimes accompanied by a multiplication chart for base five which contains the 1 digit by 1 digit multiplication facts and/or a chart which indicates what 
types of units are produced (see Figure 12). This second chart may leverage student generated generalizations from Task 2 about multiplication by the base.

\begin{tabular}{|c|c|c|c|c|c|c|c|c|c|}
\hline & 0 & 1 & 2 & 3 & 4 & & & & \\
\hline 0 & 0 & 0 & 0 & 0 & 0 & & Ones & Longs & Flats \\
\hline 1 & 0 & 1 & 2 & 3 & 4 & Ones & Ones & Longs & Flats \\
\hline 2 & 0 & 2 & 4 & 11 & 13 & Longs & Longs & Flats & Long flats \\
\hline 3 & 0 & 3 & 11 & 14 & 22 & Flats & Flats & Long flats & Flat flats \\
\hline \begin{tabular}{|l|}
4 \\
\end{tabular} & 0 & 4 & 13 & 22 & 31 & \multirow{2}{*}{\multicolumn{4}{|c|}{$\begin{array}{l}\text { b) This chart indicates which type of unit is produced } \\
\text { when two units are multiplied. For example, when a } \\
\text { long is multiplied by a flat, a long flat is produced }\end{array}$}} \\
\hline jase & & & & & $\begin{array}{l}\text { or } \\
\text { chart }\end{array}$ & & & & \\
\hline
\end{tabular}

Figure 12: The standard algorithm for multiplication is sometimes accompanied by one or both of these student generated charts.

- Partial Products strategy. PSTs may make use of the distributive property explicitly to create a partial products strategy. For example, a PST may choose to split apart the problem $42_{\text {five }} \times 31_{\text {five }}$ to find $10_{\text {five }}$ copies of $31_{\text {five, }}$, (which is $310_{\text {five }}$ ), repeat this 4 times (i.e. $4 \times\left(10_{\text {five }} \times 31_{\text {five }}\right)$ ), and then to add on 2 more copies of $31_{\text {five. }}$ (i.e. 4 x $\left(10_{\text {five }} \times 31_{\text {five }}\right)+\left(2 \times 31_{\text {five }}\right)$. Likewise, PSTs may discuss splitting up the rectangle into helpful sub-rectangles, rather than using repeated addition language. The choice to split up the problem may be made with explicit awareness of the underlying distributive property, or PSTs' use of the distributive property may be more implicit. For example, PSTs may make implicit use of the distributive property in the standard algorithm or in subgrouping within the repeated addition model.

First follow-up question. Tasks 1 and 2 relied on the repeated addition model. The first follow-up question encouraged PSTs to link their prior work with the repeated 
addition model to the idea of area as an emerging model of multiplication. I anticipated that PSTs would be able to make a connection between the two models by imagining the area split into rows of size one. The multiplier (which indicates the number of groups) is represented as the number or rows while the multiplicand (which represents the size of the groups) is represented by the width of the rows (see similar imagery in Figure 9).

The first follow-up question also provided an opportunity to discuss the differences between the two models for multiplication. In particular, the repeated addition model serves as a gateway from addition to multiplication, while the area model lends itself to a wider array of multiplication problems, including larger whole numbers, fractions, and algebraic expressions.

Second follow-up question. The second follow-up question focused on unpacking the distributive property within PST generated solutions, emphasizing that this property is already in play in the students' own work. The distributive property is not a teacher generated concept, but rather a student generated concept that the teacher helps to name using appropriate mathematical language. The prompt for the follow-up question leaves a blank for the teacher/researcher to select a PST generated solution strategy to leverage to highlight the distributive property. One potential strategy that would serve the intended purpose of focusing on the distributive property is the strategy of filling the rectangle with base five manipulatives (Figure 13a). 


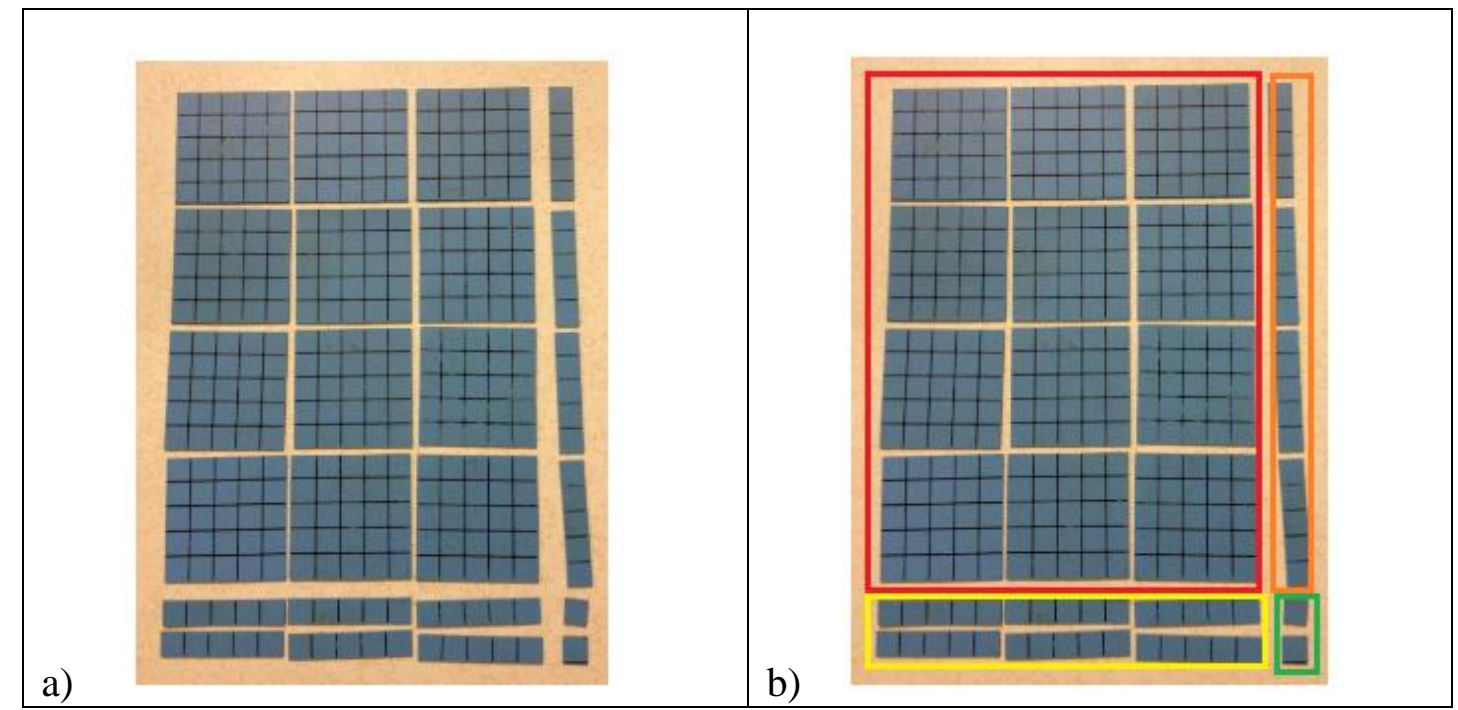

Figure 13: The rectangular image is filled with base five manipulatives, which can be partitioned into sub-rectangles.

By focusing on how the image is split into different quadrants based on the types of manipulatives used (see Figure 13b), the subsections of the rectangle could be linked with the dimensions along the sides of the rectangle. The subdividing of the rectangle corresponds to subdividing the linear dimensions along place value lines. For example, the top of the red rectangle corresponds to the $30_{\text {five }}$ in $31_{\text {five }}$, while the top of the orange rectangle corresponds to the $1_{\text {five }}$ in $31_{\text {five. }}$. The left side of the red rectangle corresponds to the $40_{\text {five }}$ in $42_{\text {five }}$, while the left side of the yellow rectangle corresponds to the $2_{\text {five }}$ in $42_{\text {five. }}$

After noting that the rectangle has been split up, the follow-up question asked: Why is that allowed?, Why is that useful?, Is that always an option?, and What is it called? I anticipated that this series of questions would help the PSTs to bring the idea of the distributive property from the background to the foreground of their thinking. By focusing on the utility of the idea of splitting up a multiplication problem into sub- 
problems (partial products strategy), the PSTs are primed to be more aware of the distributive property in their generalized strategies, which are the focus of task 4.

\section{Task 4: Building a General Multiplication Strategy}

Mathematical Goals of Task 4. The sequence of tasks culminated with the development of a general solution strategy in Task 4 (Figure 14). In addition to the explicit mathematical goals of building a general solution strategy and linking that strategy to visual models for multiplication, I also wanted PSTs to reflect on the process they have undertaken. I wanted PSTs to consider the reinvention of algorithms to be feasible for themselves and for elementary school students (in terms of both class time and mathematical ability). I wanted PSTs to reflect that when students develop their own strategies for multidigit operations they find more than the answer to a specific problem. They also build understanding of mathematical structures, ownership of knowledge, and understanding of number properties (e.g. distributive property). The specific goals for Task 4 are listed below.

- Create a general strategy for base five multidigit multiplication problems.

- Focus explicit attention on the role of the distributive property and the role of place value in the development of efficient strategies.

- Reflect on the process of creating general strategies, both for themselves as students and for their own future elementary school students.

Rationale for the Design of Task 4. The box of rectangles task was intended to move the PSTs from needing a solution to a single problem to needing a solution for a type of problem. This task serves as the prompt for reinventing a general solution 
Investigating Alternate Numeration Systems

strategy for multidigit multiplication. The rationale for the context of area was described

in the earlier section on didactic phenomenology within Task 3 (see Figure 2-13).

\section{Task 4: Part 1}

Alice and Bob, who live in the land of Base Five, have a large box of rectangles. They need a strategy to find the area of any rectangle in base five. Your task is to create and explain an efficient strategy that will work to multiply any two base five numbers. Record your strategy on the provided paper. [Students will be asked to record in pen and will be given new colored papers for various drafts]

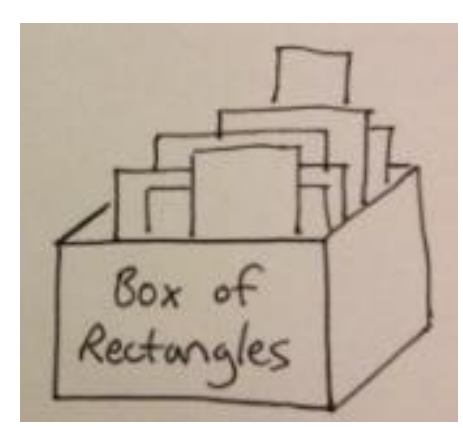

[As part of this task, I have a box of rectangles (cut out of cardstock) with various dimensions that correspond to whole numbers, if the rectangles are measured using base five manipulatives ( 1 unit $=1 \mathrm{~cm}$ ). I may include some index cards that list the dimensions of rectangles that I have not built, to ensure that strategies do not rely only on physically filling the rectangle]

List of rectangles in the box (in base five):

$(42 \times 31$ was task 3$)$

$\begin{array}{lllll}12 \times 23 & 22 \times 23 & 11 \times 11 & 21 \times 34 & 24 \times 24\end{array}$

$\begin{array}{lllll}30 \times 14 & 100 \times 12 & 103 \times 23 & 120 \times 34 & 20 \times 30\end{array}$

Index cards: $44 \times 23,102 \times 24,234 \times 20, \quad 234 \times 21$

Reflection:

- Describe the process you and your group went through to create a general multiplication strategy. Note: I'm not asking for your final general strategy, I want you to reflect about the creation process and how you moved from solving the specific problem $3 \times 23$ to then inventing a strategy for any base five multiplication problem, in just a few days.

- Reflecting as a teacher, do you think it would be possible for $4^{\text {th }}$ graders to invent a strategy for multiplication in base ten (if they had never seen the U.S. standard algorithm)?

Figure 14. Task 4 focuses on a general strategy for multiplication in base five.

The primary mathematical goal of the task was for students to create a general strategy for multidigit multiplication, based on the emerging model of partial products.

The area context of rectangles provided a physical context for situated strategies involving splitting the rectangle into sections that can be filled with different base five unit types. A mathematical observer may recognize that the way students may organize 
Investigating Alternate Numeration Systems

(or mathematize) the area context corresponds to a partial products strategy (model of partial products). However, it is possible that students may not articulate the intention behind splitting up numbers along place value until they shift from describing particular solutions to specific numeric problems and shift toward explaining a more general strategy (model for partial products).

As the students shifted from describing particular rectangle solutions to more general strategies based on place value, they would make a vertical shift in their mathematizing, leveraging the partial products strategy as a model for creating an efficient algorithm for multiplication.

Anticipated Student Mathematical Activity for Task 4. The first 3 tasks had been piloted previously, providing me with a good deal of insight into potential student mathematical activity as students engage with the tasks. Task 4 , on the other hand, had not yet been piloted; therefore the following anticipated student mathematical activity was more hypothetical in nature. Previously, after attempting a two digit by two digit multiplication problem, students were given a 2 digit by 3 digit problem, which was followed by a class discussion of strategies for the particular numeric problems given. The new Task 4 invited PSTs to create and explain a general strategy for multidigit multiplication.

I anticipated that PSTs would create a series of strategies for multidigit multiplication, beginning with specific strategies for a particular problem or type of problem and moving toward broader classes of problems. For example, initial strategies might be appropriate only for two digit by two digit problems in which there are no zero digits. Later strategies might be applicable to larger numbers or numbers which contain 
zeros as digits. I anticipated that strategies would increase in efficiency and generality and that students would begin to justify why their strategies are appropriate, efficient, and general. One goal of the study of this task was to determine how the distributive property could appear in student strategies and how to leverage this modifications of the instructional sequence.

The design heuristic of emergent models provides language to discuss shifts in students' mathematical activity as they move from situated strategies to more general strategies. This task was designed so that student mathematical activity would result in the emergence of the model of the partial products strategy for multiplication. The partial products strategy for multiplication refers to the idea of splitting a multiplication problem into sub-problems, computing the products for the sub-problems, and summing them to reach the total. Figure 15 provides visual and symbolic examples of this strategy.

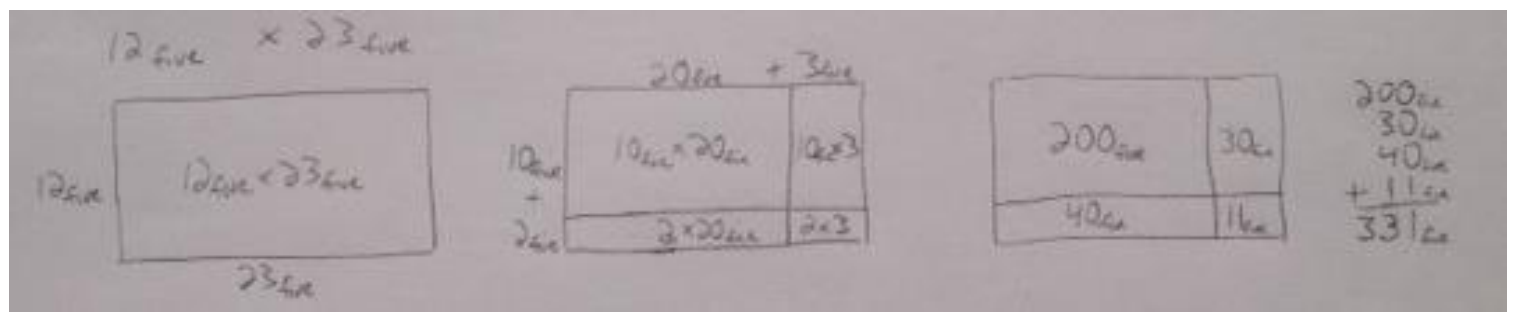

Figure 15: The multiplication problem $12_{\text {five }} \mathrm{x} 23_{\text {five }}$ is split along place value lines to create 4 sub-problems. Each problem is solved separately and the partial products are then summed.

PSTs were asked to apply their general strategy descriptions to other rectangles in the box of rectangles. Particular rectangles could be selected from the box to push on strategies which are only appropriate to a subset of possible multiplication problems. As the teacher, I could encourage an explicit focus on connecting the lengths of the sides of the rectangles with the area, particularly as the area is subdivided and the lengths of the sides are correspondingly subdivided. I anticipated that one strategy that would emerge 
Investigating Alternate Numeration Systems

would be to split the rectangle into four pieces, with the quantity four being a focus, rather than the convenience or efficiency of the pieces being a focus. For example, in 30 x 23, splitting the problem into 2 pieces, $30 \times 20$ and $30 \times 3$, may make more sense than 4 pieces. Meanwhile, in $123 \times 23,6$ pieces might be more advantageous.

\section{Pre / Post Survey Questions}

In addition to the four tasks described for the teaching experiment, I also included several pre/post questions, using a combination of written surveys and interviews.

\section{Pre-questions about Algorithms}

- Show me how you would solve $345-186,48 \times 34$, and $752 \div 4$.

- The step-by-step strategies you used to solve the two problems are called algorithms. Where do algorithms like this come from?

- Do you remember how you learned about algorithms like these?

- Do you know a different way to solve $345-186,48 \times 34$, or $752 \div 4$ ?

- Would it be reasonable to have $4^{\text {th }}$ graders invent their own algorithms for problems like $345-186,48 \times 34$ and $752 \div 4$ ? Why or why not?

$\circ$ (If yes, what would those algorithms look like?)

\section{Post-questions about Algorithms}

- Where do algorithms for operations (addition, subtraction, multiplication, division) come from?

- How have your ideas about algorithms changed since the start of this course?

- Would it be reasonable to have $4^{\text {th }}$ graders invent their own algorithms for problems like $48 \times 35$ and $542 \div 6$ ? Why or why not? 
Investigating Alternate Numeration Systems

I anticipated that the pre / post questions about algorithms would show some shifts in PSTs views about the role algorithms. Initially PSTs might have a static view of algorithms as something fixed and created by others, prebuilt and unquestioned. After the teaching experiment, I anticipated that PSTs would begin to show a shift toward a view of algorithms as sense-making processes which are created by people such as themselves and their students.

\section{Questions about Properties:}

- What do you think of the distributive, associative, and commutative properties?

- Record what you can remember about these 3 properties, as well as what you think of them.

- Should these properties be taught in elementary school?

- What are possible reasons these properties should be a part of the elementary school curriculum?

- What are possible reasons these properties should not be taught until later?

- (Possible follow-up) As an example, let's focus on the distributive property. It is sometimes written as:

$$
a \cdot(b+c)=a \cdot b+a \cdot c .
$$

○ Do you remember learning about this property? What sort of math was it used for? Can you think of some examples of how you might use this property in elementary school?

I anticipated that these interview questions would reveal that prior to the teaching experiment, the PSTs were not comfortable with the properties and would not be able to correctly identify them or see their utility in elementary mathematics. I anticipated that at 
the end of the teaching experiment, the PSTs would have a different view about the distributive property, seeing that it is at the heart of efficient algorithms for multiplication. 